(I) $(1)=$

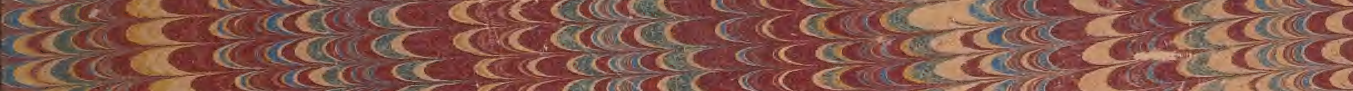

ane S -

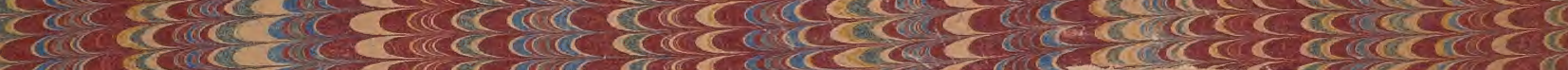

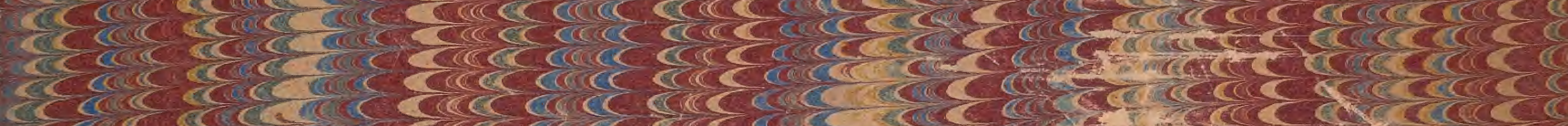

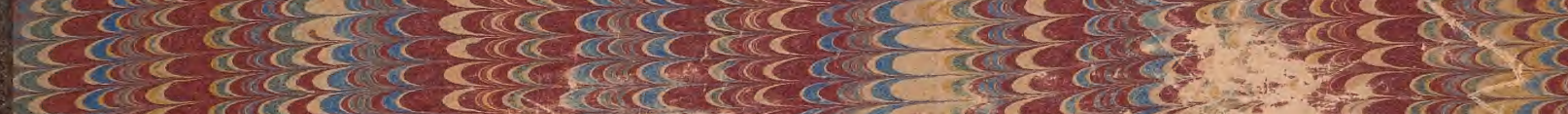

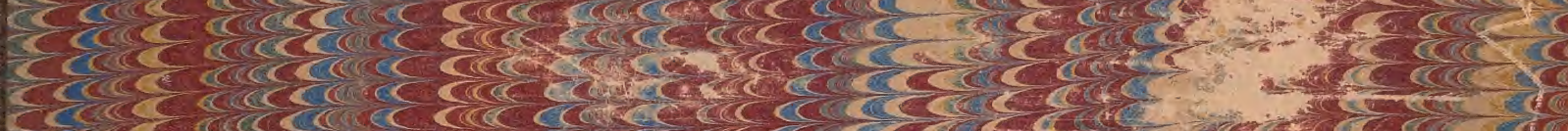

-

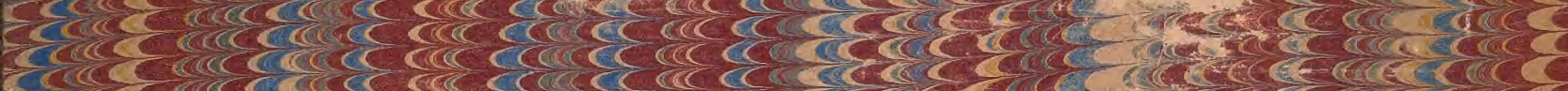

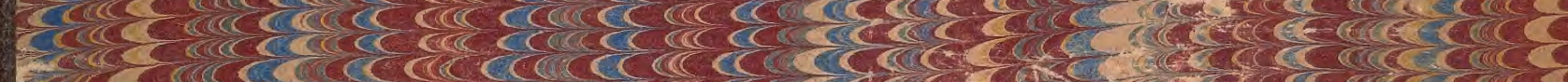

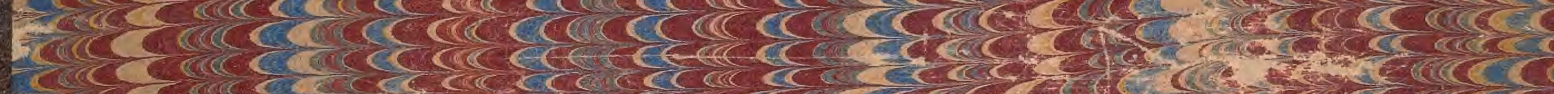

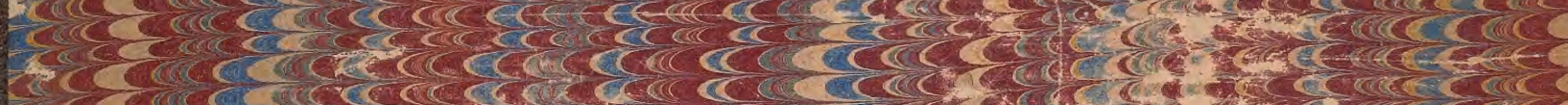

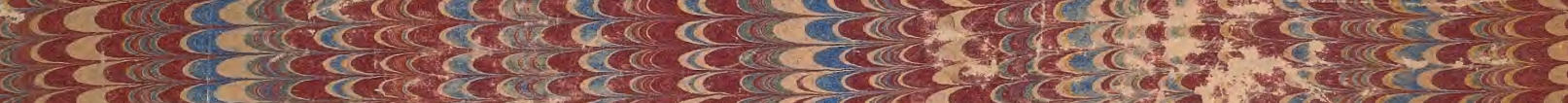

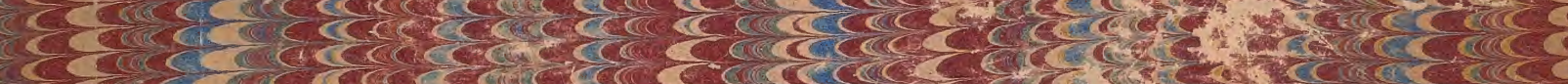

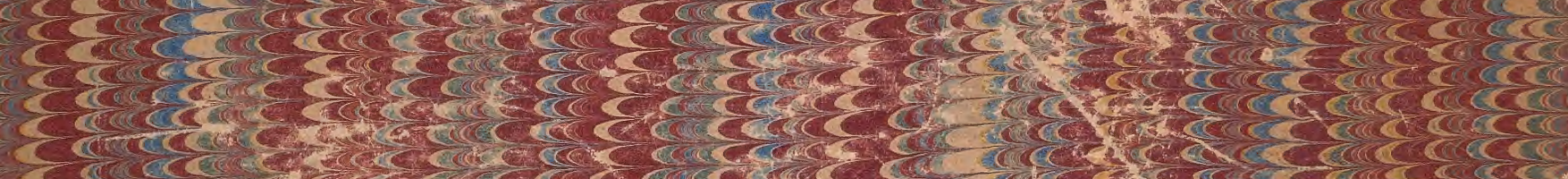

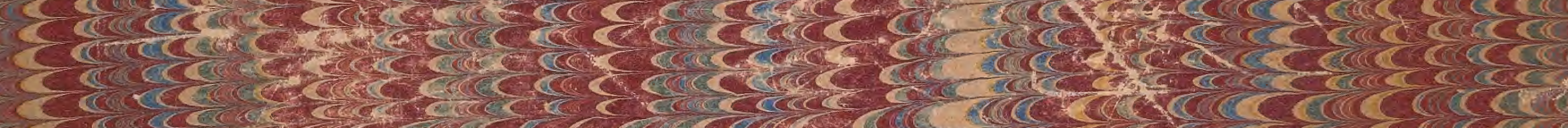

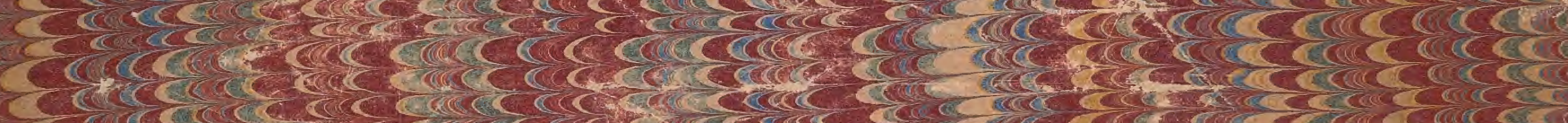

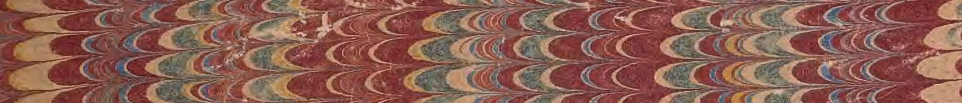

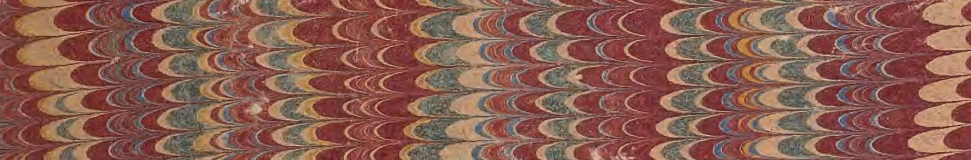

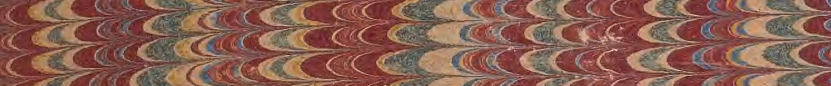

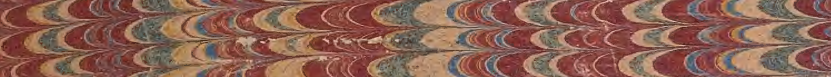

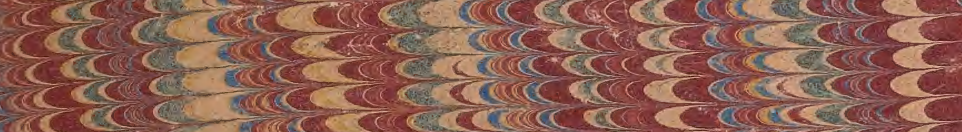

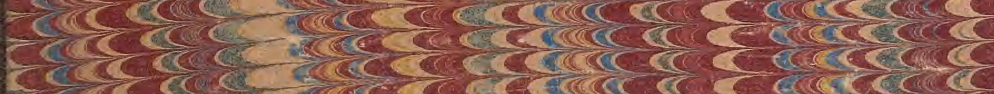

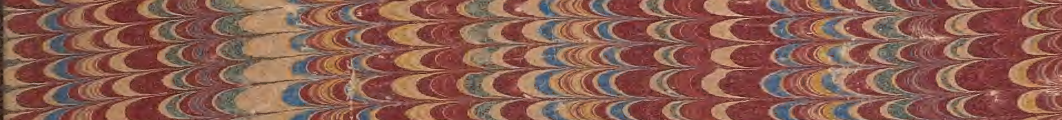
mancentere हो

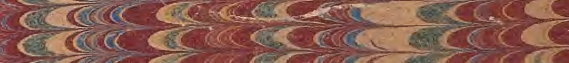

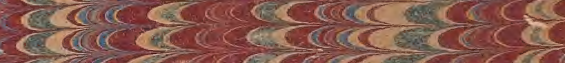

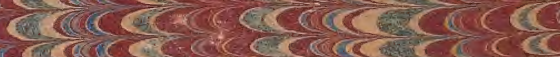

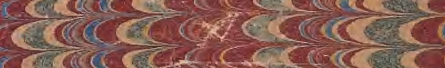

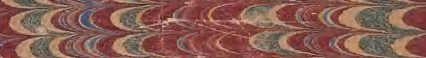

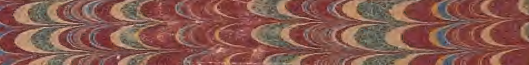

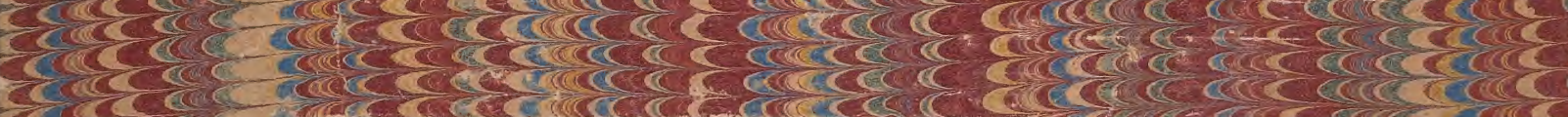

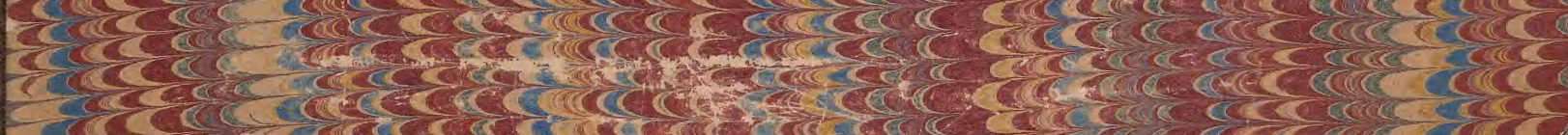

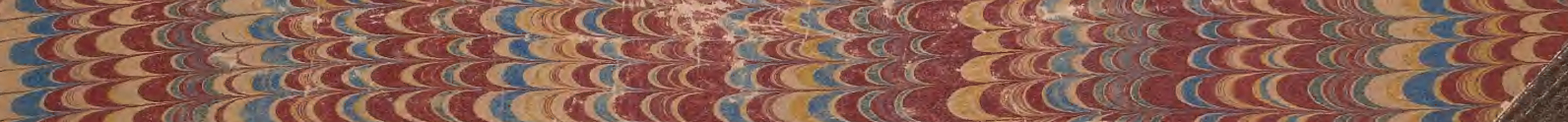

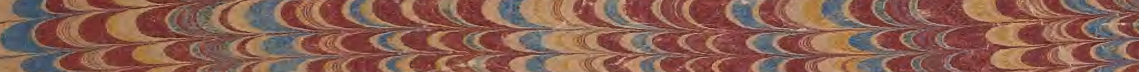

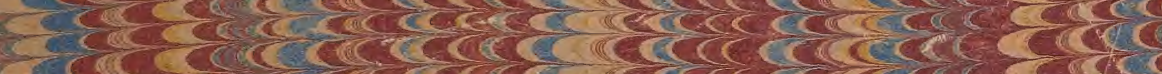

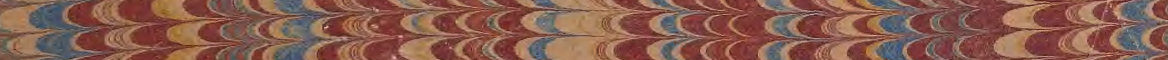

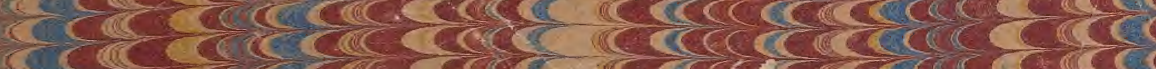

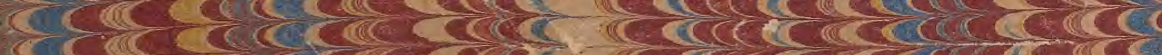

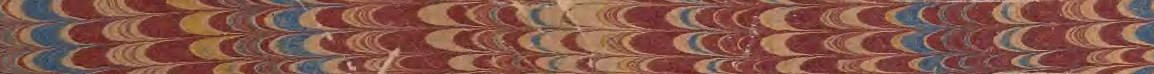

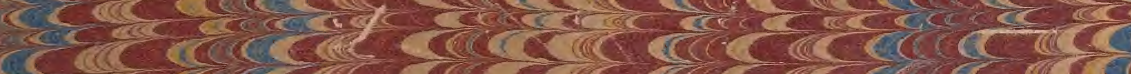

c(c) cesess ces?

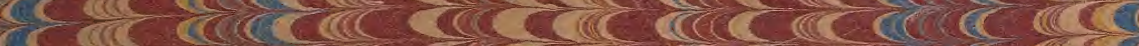

ताले त्रे $\operatorname{cosec}$ c) 


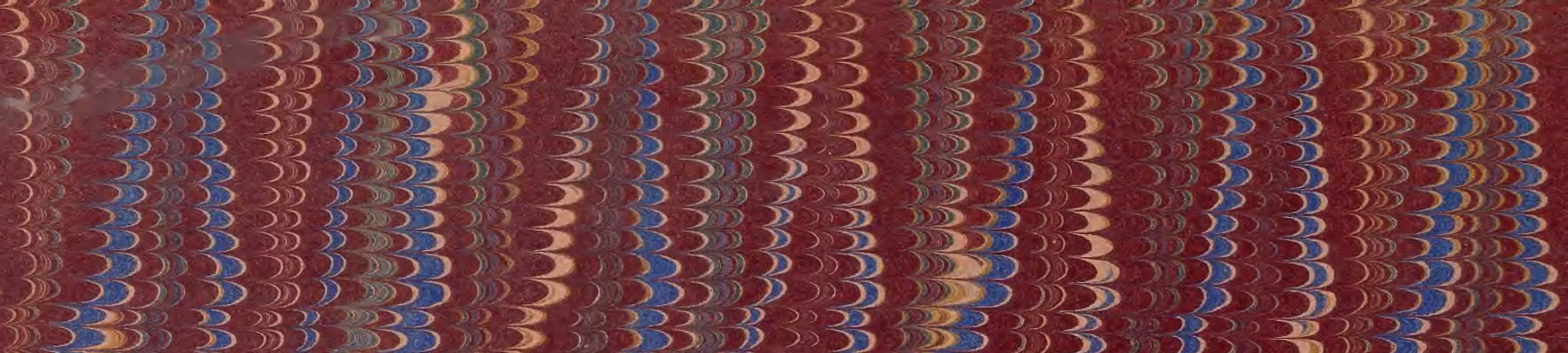

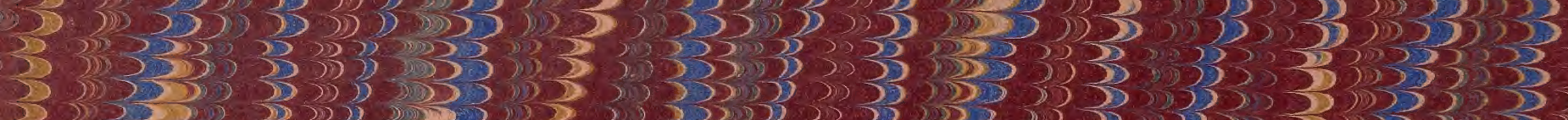

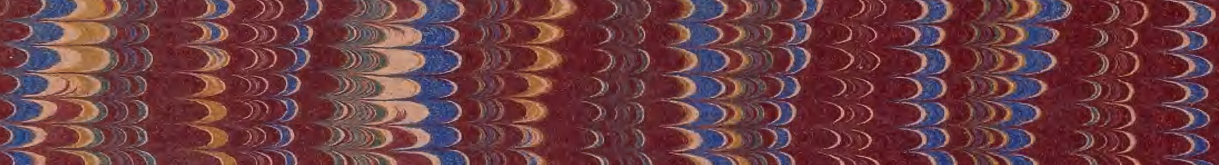

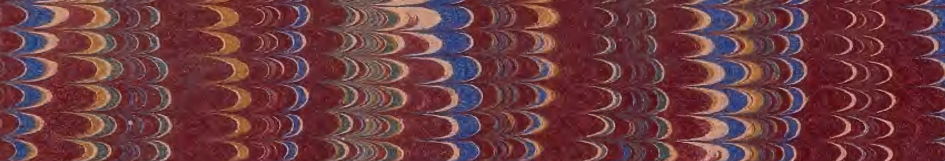

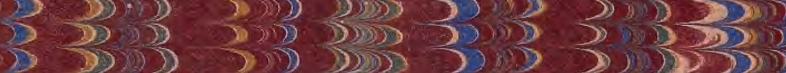

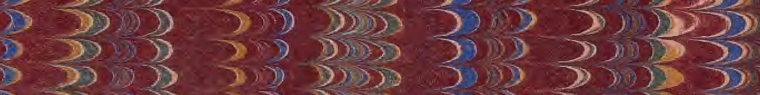

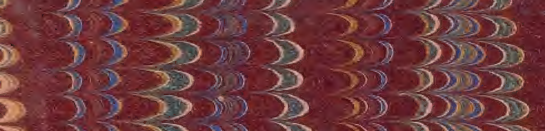

\section{(}

.

3

3

33

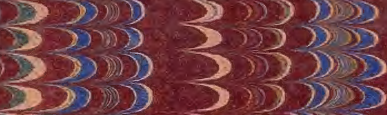

(3) 35,0303

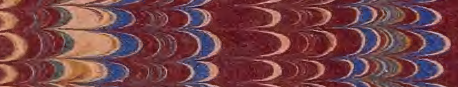

333333333

3.3333333

$3.33,35353$

$33,3,23$

) $)>3$

3

33333

33233

(3) 33 S ग

33,30353

3303 (3)

3

333

3.
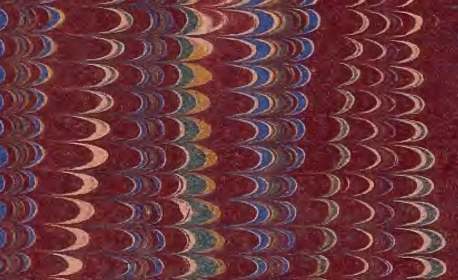

J) $>3$

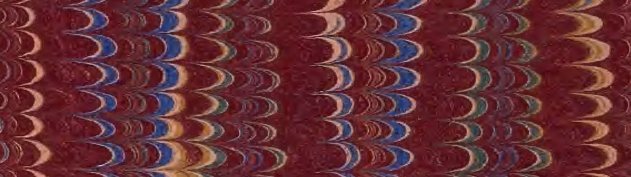
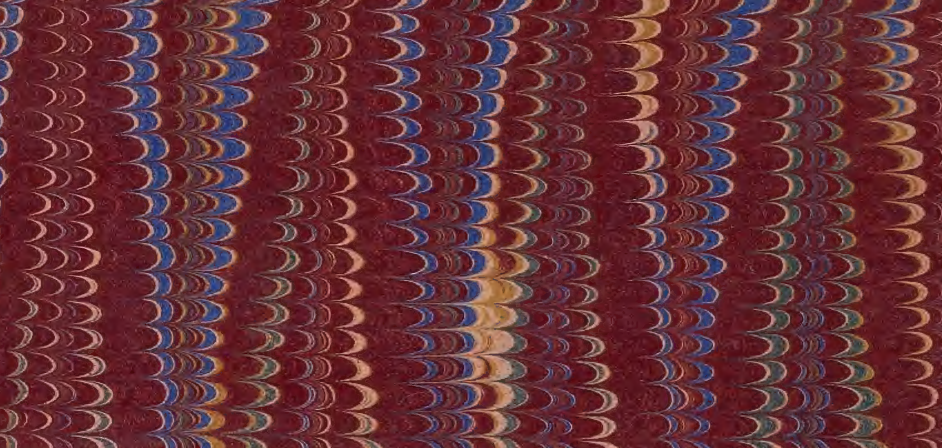

$33,33,3333$

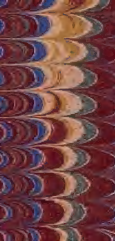

(B) 3003

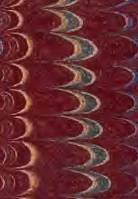

$3 ., 30,3$

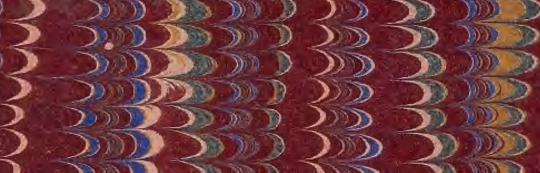

3203

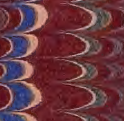

33 , 3

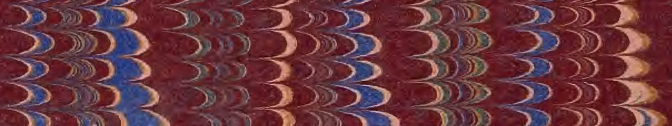

$32,33,3,333533$

3
3
3
3
3

i)

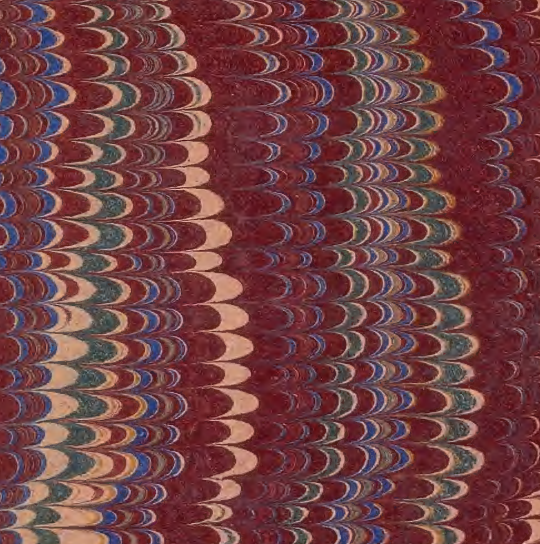

(3) 353

39333

3.3353

32032

D) D) $3>3$

ᄀ) $>325$

(3) 23

() 25

()) 20
(

$323,3,33053235$

$3>33,3233$

$\begin{array}{lll}3 & \infty\end{array}, 3,3,33$

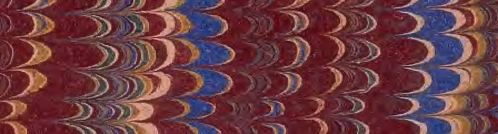

3 3is 33,35

$3 \rightarrow 3,3,3 i m$

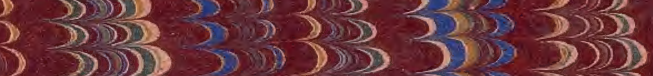

) DDD

33

3

3.

3

.

3,33

323323

$30,350,33,35$

$3) 33$ 3 333

3) $)(3,33) 3$

3) 3230353

3)10303 323

3) 3,3333

D) $)>3 \rightarrow 3$

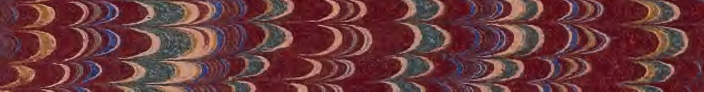

33

33

333,33

3) $3>3333$
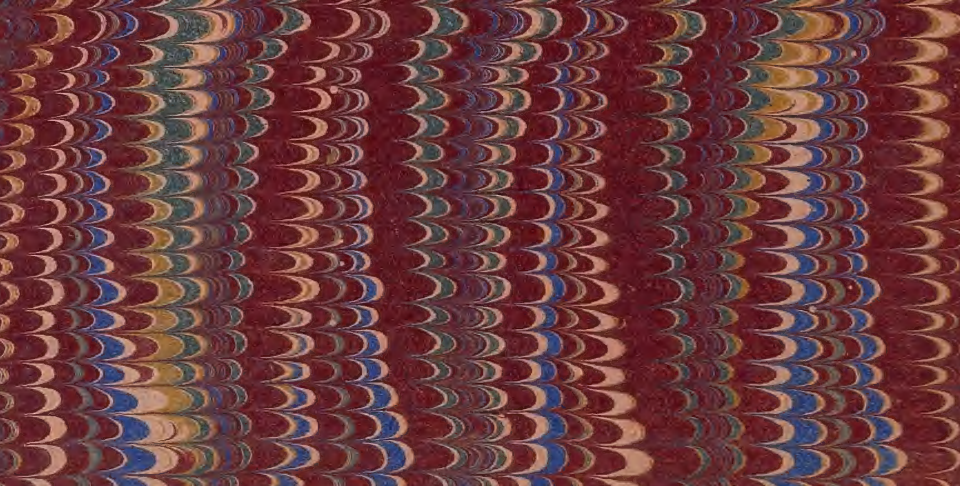

3 .)

5.7

3

S)

323

3. 3 (i) i) 


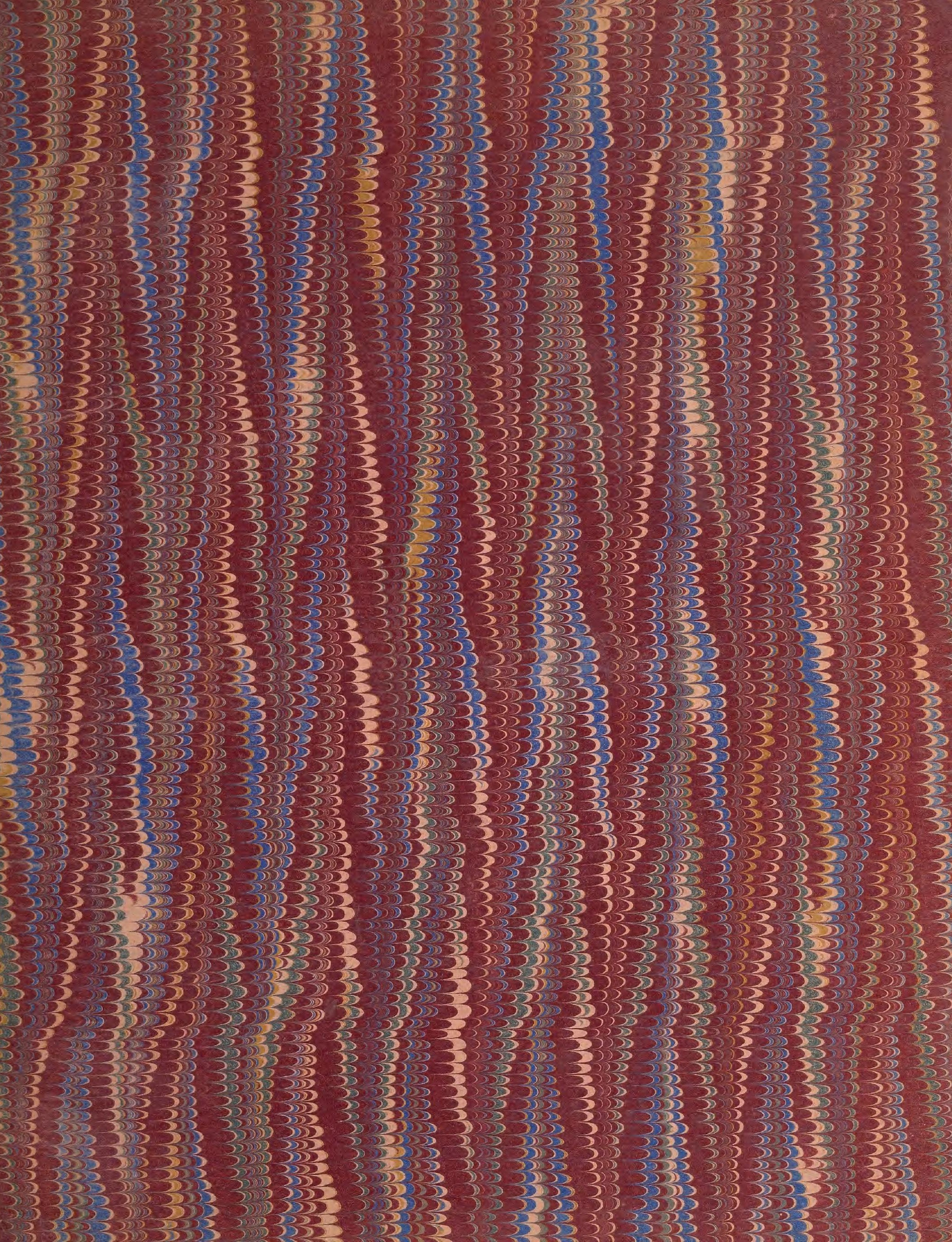









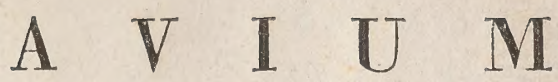 \\ SPEGIES NOVAE,
}

QUAS

IN ITINERE PER BRASILIAM ANNIS MDCCCXVII - MDCCCXX

JUSSU ET AUSPICIIS

\section{A X I M I LIA N I J O S EP II I I.}

\section{B A V A R I A E R E G I S}

\author{
SUSCEPTO \\ COLLEGIT ET DESCRIPSIT \\ Dr. J. B. DE SPIX,
}

Ordinis Regii Coronae Bavaricae civilis Eques, Academiae scientiarum Bavaricae Socius ordinarius, Musei Regii zoologici, zootomici et ethnographici Conservator, Academiae Naturae Curiosorum, Societatis literariae Edinburgensis, Moscoviensis etc. Sodalis.

tabulae CXVin a M. SCH Mid Monacenst sculptae.

T O M US II.

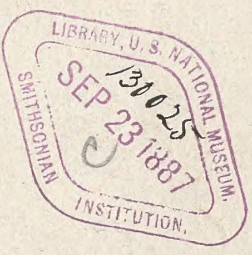

$\begin{array}{lllllllll}\text { M } & \text { O } & \text { N } & \text { A } & \text { C } & \text { H } & \text { I } & \text { I. }\end{array}$

TYPIS FRANC. SERAPH. HÜBSCHMANNI.

MDCCCXXV. 



\section{$I N D E A$.}

T O M U S. II.

H I RTININES.

Capnimugues longicaudatus.

rupestris

hirundinaceus

Leucopygus

A MTELIDES.

Casmarhynchus ecarunculatus Antrelis Carnifex adult.

\section{PIPRAE.}

PrPRA

\section{cornuta}

coronata

caudata. MI. F.

Filicauda, IM. F.

elata

herbacea

INI USCICAPAE.

Todes.

PLatyruxncuus Xanthopygus

cinereus. IM. F.

Ruficauda

Chrysoceps

sulphurescens. INI. F.

hirundinaceus

cinereus

Filicauda

Flaviventer

Brevirostris

paganus

murinus

MuscicapA

\section{Longicaud}

Vetula

furcata

sulphürea

cinerascens. INI. F

velata

Joazeiro

Polyglotta

similis

thamnophiloides

ciricrea
Pagina 1 Tabula 1

- 2 - 2

- " - 3

$3-4$
$-\quad 4-5$

$\begin{array}{llll}\text { - } & 5 & - & 7 \\ - & { }^{\prime} & - & { }^{3} \\ - & { }^{6} & - & 6 \\ - & 0 & - & 8 \\ - & 7 & - & 8^{\circ} \\ - & 6 & - & 8\end{array}$

- 8 - 10

- 9 - 99

- " - 11

- 10 - "

- , - 12

- $11-13$

- " - "

- $12-14$

- " - 15

- $13-15$

- $\quad$ - 16

- $14-16$

- , $\quad-17$

- $15-18$

-,$\quad-19$

- $10-20$

- " - 21

- $17-22$

- , - 23

- $18-24$

- " - 25

- $19-20$ 
IM U S CICA PA.

galeata. MT.

galeata. F.

Fulvicauda

nivea

dominicana

Albiventer. M. F.

rufina. IM. F.

LANII.

mystacea. IM. F.

Thamnophus Albiventer. IV. F. lineatus. IM. F,

agilis

affinis

guttatus

radiatus. IMT.

radiatus $F$.

strigilatus

stellaris

liuficollis

albonotatus. MT.

albonotatus, F.

IMelanoceps

Leuconotus

griseus. F. .

griseus. IMI.

striatus

gularis

myotherinus, IM. F.

Melanogaster

F I I N ILLAE.

Pachynunchus variegatus

Cajanus. IVI.

semifasciatus. MT

Cuvieri

niger

cinerascens

rufescens

TañGra nigrogularis. IM. F

Saira. IM.

viridis. IM.

penicillata

brunnea. IVI.

Rufiventer

Aurifrons

Schrankii. IM. F.

Auricapilla. IM. F.

Cristatella

Ruficollis

Pagina 20 Tabula 27

-

—

- $21-29.2$

- " - 30

- $22 \quad$ - 31

- $23-32$

- $24-33$

- $25-34$

$\begin{array}{lll} & 26 & \end{array}$

- 24 - ,

- $24-38$

- $20-36$

- $27-\quad$

- $\quad-37$

- " - "

-38
$-\quad 28-39$

- " - "

- $\quad 41$

- " - 40

- $30-41$

-,$\quad-42$.

- $31-43$

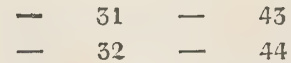

-,$\quad-44$

- $33-45$

- 34 -

$\begin{array}{llll}- & \text { - } & \text { - } & \\ - & 35 & - & 47\end{array}$

-,$\quad$ - 48

- $30-48$

- " - 49

- 37 - 49 .

- $\quad$ - 50

- $38-50$

- " - 51

\begin{tabular}{l}
$-39-52$ \\
\hline
\end{tabular}

- $40-53$ 


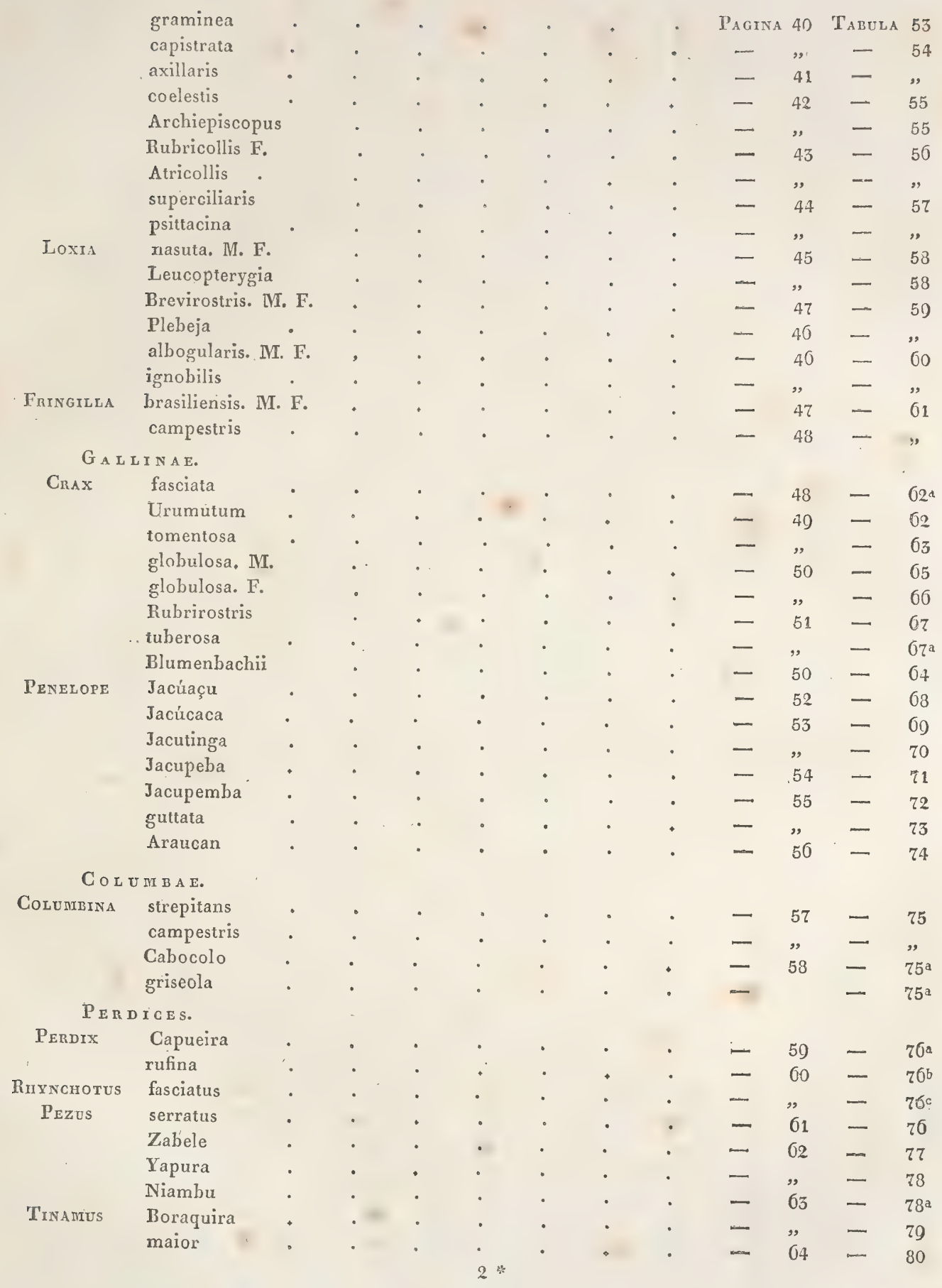




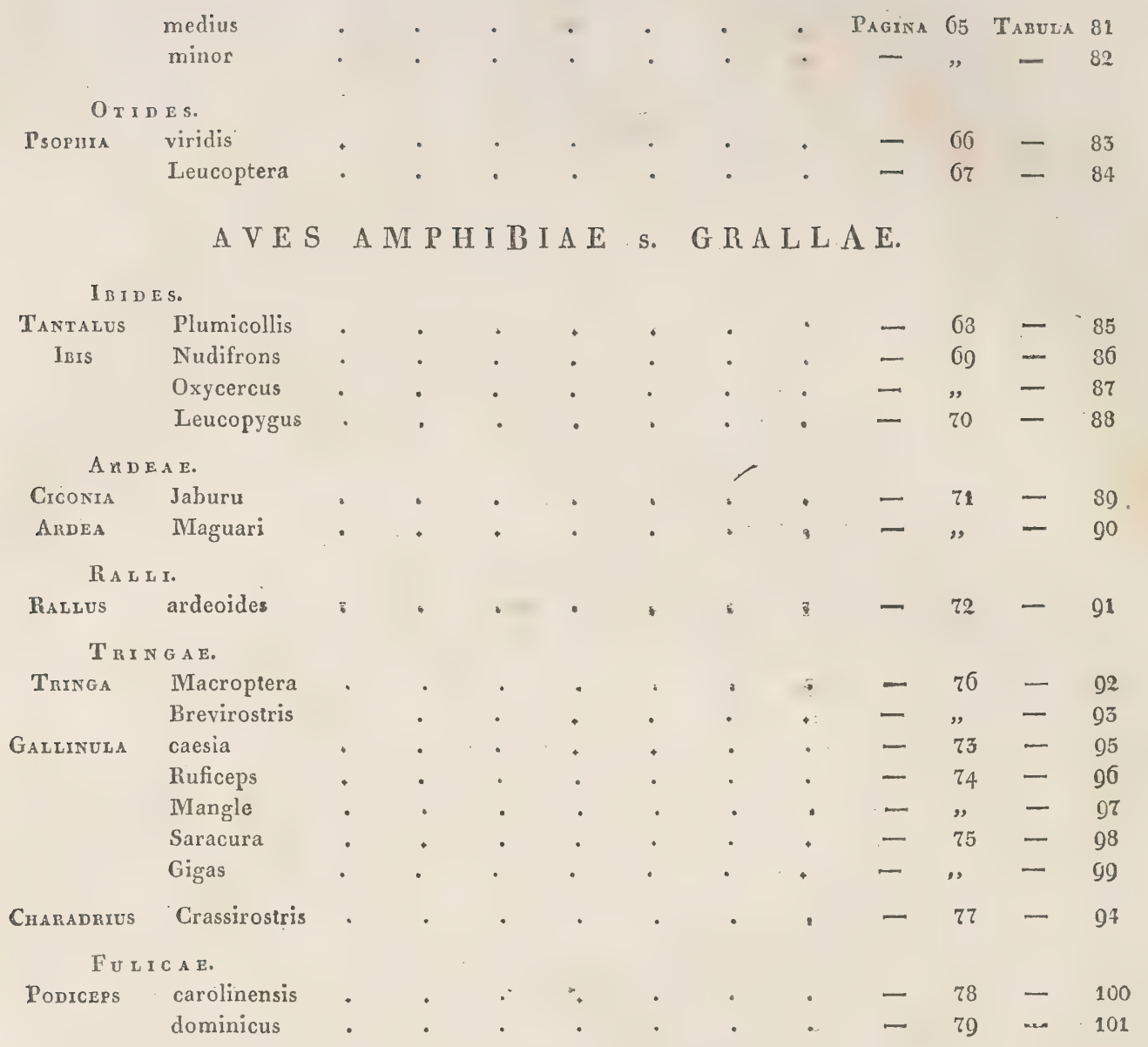

\section{A VES A Q A T ICAE.}

L A R.

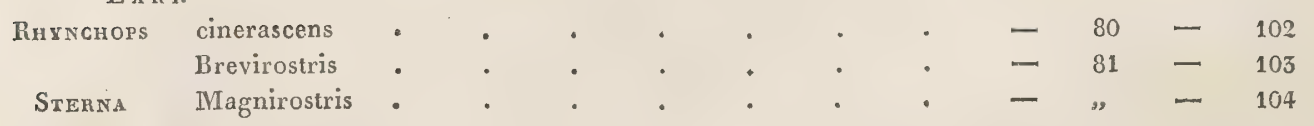

PELECA N.

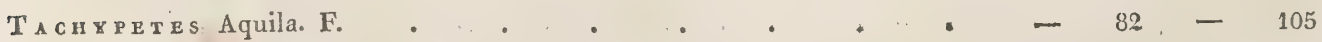

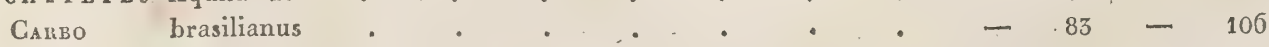

Sula brasiliensis . . . . . . . . . $\quad$. $84-107$

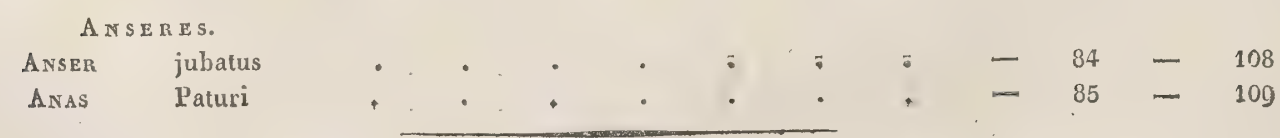




\title{
AVES TERRESTRES.
}

\author{
F a m i l i a: H i ru n d i n e s.
}

Plani- et brevi- rostres, insectivori, in locis tenebrosis, petrosis, vel in terra nidificantes; capite rostroque

dentato depressis; collo brevi; oculis praegrandibus; naribus basalibus; rotundis; rictu oris peramplo; tarsis abbreviatis; alis vel cruciatis vel subdecumbentibus.

\section{GenUs 1. C A P R I M U L G US.}

Striginus, nocturnus, in terra nidificans, strigum modo et haud alte volans; rostro basí depresso, vibrissis tecto, apice tenui, uncinato, introrsum canaliculato, rictu oris amplissimo, angulato-sinuato; plumis corporis intumidis; digitis lateralibus brevioribus, subsemipalmatis, postico frequenter minuto; ungue digiti medii incurvo, margine interiore plerumque serrate.

\section{Speries 1. CAPRIMULGUS LONGICAUDATUS. Taв. I.}

Maior, supra subtusque ferrugineus nigroque maculatus; pectore subtus Iunate nigro-ocellato; cauda alis praelongiore, subgradata; tectricibus posthumeralibus albicantibus; remigibus rectricibusque rúfis, nigro-fasciatis; tibiis vix nudis; ungue digiti medii non serrato.

$\mathrm{D}$ ESCR IPT10. Corpus C. cayennensi vix minus, tenuius vero, supra et subtus ferrugineum, plumulis transversim tenere nigro undulatis, medioque longitudinaliter nigro decussatis; caput fuscescens, plumulis lateraliter rufo-fasciolatis, striga inframaxillari utrinque nigra; nucha dorsumque rivaciter rufescentia; tectrices alarum elongatarum posthumerales axillaresque albae, scapulares et radiales nigrae, mediae fulvo-ferrugineo nigroque undulatae vel maculatae; remiges rufo nigroque fasciatae; cauda alis praelongior, subgradata, rufa, nigro punctulata medioque 8 fasciata; corpus subtus rufescens, nigro lineariter reticulatum, inter pectus et abdomen ocellis nigris fulvisque semicirculariter notatum; crissum ochraceum, fere immaculatum; uropygium rufum nigroque undulatum; rostrum fulvescens, infra oculos planum, apice tenue, uncinatum; nares plumis obtectae; oculi grisei; tarsi breves, validi, vix nudi, fulvescentes; digitus lateralis internus, praecipue posticus fimbriati; unguis digiti medii non serratus. Longitudo corporis $1^{\circ}, 6^{\prime}$, caudae $10 \frac{\pi^{\prime}}{2}$, c. alis 1 . $4 \frac{3}{4}$, , tarsi $4^{\prime \prime}$, rictus oris $2^{\prime}$.

Habitat in sylvis A. Japurae. 


\section{Stecies 2. CAPRIMULGUS RUPESTRIS. Taв. II.}

Minutus, albescens; gula, abdomine, alis mediis caudaque breviter bifurcata latere albis; pectore obfuscato; tarsis dimidio nudis; ungue digiti medii serrato.

$\mathrm{D}$ ESCripto. Corpus Merula minus, supra et in pectore albo-griseum, maculis nonnullis nigris cruciatis, subtus niveum; caput griseum, vix rufescens, longitudinaliter nigro-lineatum; dorsum uropygiumque fulvescenti-grisea, fusco tenerrime undulata vel reticulata, lineis nonnullis cruciatis nigris, plumis basi nigris, in collari albis; tectrices alarum anteriores pallide fulvescentes, nigro, rarius albo maculatae, posteriores seu longiores griseae, nigroque lineolatae ac crucialae; remiges primariae longae, cruciatae, nigricantes, secundariae niveae, a pice nigro fasciatae; alae subtus inedio et versus axillas albae; cauda alis brevior, subbifurcata, supra grisco-fusca, sublus nivea, apice nigricans; rectrices intermediae griseo-fuscae, lineolis nigris undulatae, laterales medio et basi albae, apice nigricantes, extima margine exteriore basali nigricante; gula, pone aures, abdomen, crissum femoraque nivea; pectus obfuscatum, nigricanti subundulatum; tarsi dimidii albo-lanuginosi; digiti breves, vix fimbriati, ungue medio serrato; rostrum minutum, fuscescens, vix aduncum, vibrissis fere nullis; pedes fusci. Longitudo maris foemina maioris $8^{\prime}$, caudae $3 \frac{3}{4}^{\prime}$, tarsi $6^{\prime \prime}$, rostri $4^{\prime \prime}$.

Habitat familiaris in insulis petrosis $\mathrm{fl.} \mathrm{Nigri,} \mathrm{diu} \mathrm{haud} \mathrm{raro} \mathrm{volitans.}$

\section{Speres $3 \cdot+$ CAPRIMULGUS HIRUNDINACEUS. Taв. III. F. 1.}

Minutus, brunneo-fuscus; suhtus rufo nigroque fasciatus; tarsis lanuginosis; remigibus medio vix, et rectrice exteriore fasciata apice introrsum albis; ungue digiti medii serrato; cauda aequali; gula non alba.

$\mathrm{D}$

Escriptro. Corpus Emberiza vulgari vix maius, supra brunnco-fuscum nigroque lineolatum ac variegatum, subtus fulvo nigroque undulatum, versus abdomen magis rufescens; caput fusco-brunnescens, nigro longitudinaliter lineatum rufoque punctatum; dorsum tectricesque alarum ac uropygii fusca, nigro rufoque punctulato-reticulata; alae cauda breviores, subtus ad humerum et axillas rufae; remiges primariae nigricantes, medio parum albo notatae, secundariae aeque fuscae, fulvo subfasciatae;' cauda aequalis, aeque ac dorsum fusca et nigro fulvoque haud fasciata sed variegata, rectrice externa nigricante, rufo-fasciata, apice extrorsum nigricante, introrsum alba; gula pectusque fulvo nigroque irregulariter undulata, collari magis rufo-albicante; abdomen et crissum rufa, raro nigro fasciata; tarsus rufo-lanuginosus; digiti graciles, sanguineo-fusci, ungue 
medio serrato; rostrum breve, nigricans, naribus non obteclis. Longitudo corporis $6 \frac{\mathbf{x}^{\prime}}{4}$, caudae $3 \frac{1}{2}^{\prime}$.

Habitat in sylvis fl. Solimoëns.

\section{Spéres 4. TAPRIMIULGUS LEUCOPYGUS. Taв. III. F. 2.}

Minimus, brunneo-nigricans supra, subtus niger, albo-undulatus; gula alisque non albo-notatis; cauda aequali, basi introrsum albo-notata; pedibus nudis, non serratis,

D ESCRYPro. Corpus hirundinaceum, minus, supra nigro-fuscum, lineolis fulvis nonnihil maculatum, subtus nigricans, transversim albo undulatum; caput, gula, tectrices alarum dorsumque nigro-fusca, fulvo subpunctulata; remiges nigrofuscae, immaculatae, caudam fere aequantes, subtus livescentes; cauda aequalis, supra fulvo obscure fasciolata, subtus immaculate nigro-fusca, vexillo rectricum extimarum interno versus basin albo-maculato; crissum nigricans, albo fasciatum; pedes nudi, fusci, ungue medio non serrato; rostrum extus vix conspicuum, nigricans. Longitudo corporis $6^{\prime}$, caudae $3 \frac{I^{\prime}}{2^{\prime}}$, c. alis $1.1^{\prime}$, tarsi $6^{\prime \prime}$, rostri $2^{\prime \prime}$.

Habitat ad litora sylvestria fl: A mazonum.

$$
\text { F a } m \text { i } 1 \text { i a: A m p e } 1 \text { i d e s. }
$$

Planirostres, insectivori; rostro exserto, dentato, vix adunco, vibrissis vix tecto; collo distincto; naribus submediis, ovatis; tarsis haud altis; digitis lateralibus haud brevibus, exteriore basi connato, postico longo.

\section{Genus I. C A S M A R H Y N C H U S.}

Nudicollis, longisonans, solitarie arborum altissimarum cacumini insidens; fronte rostroque basali planatis; rostro ad apictm trigono, subadunco, usque infra oculos hiante; digitis validis, totis fere fissis,

\section{Speres 1. CASMARHYNCHUS ECARUNCULATUS. Tab. IV.}

Maior, immaculate niveus, circa oculos, genas et jugulum fere nudus, caruncula frontis nulla.

DéCR I т Io. Corpus robustum, supra subtusque niveum, immaculatum; caput supra planum, non carunculatum, circa oculos genasque nudum, plumulis pilosis 
obsitum, porphyreo virescens; collum subtus aeque nudum, virescens, plumulis raris nigris munitum; alae mediocres, cauda aequali breviores; tarsi digitique robustiores, sanguinolenti, digito externo vix connato, postico longo; rostrum sanguinolentum, basi planum, medio carinatum, apice dentatum, subaduncum. Longitudo corporis $9 \frac{3}{4}^{\prime}$, caudae $3 \frac{\pi^{\prime}}{3}$, tarsi $11^{\prime \prime}$, rostri $1 \frac{1^{\prime}}{3}$.

Habilat in sylvis Provinciae Riode Janeiro, voce metallica longisonans, ab incolis "Garaponga vel Ferrador" nominatus; differt a C. carunculato caruncula defficiente, a C. variegato viridi-fusco alboque non maculatus.

\section{GENUS II. A IIP E L I S.}

Familiaris; voce haud metallica; collo non nudo; rostro minus plano quam elevato, arcuato, naribus plumis vibrissisque haud obtectis; digito laterali basi connato.

\section{Species. t. AMPELIS CARNIFEX. Taв. v.}

Majusculus, purpurẹus; collo, dorso, alis caudaeque apice nigris.

Desc I т то. Corpus Merulae simile, subtus totum purpureum exceptis gula juguloque nigris; caput supra purpureo renitens, ante et pone oculos nigrum; collum, dorsumque totum nigerrima; tectrices alarum posteriores sive longae nigrae, anteriores nigricantes, remiges primariae nigro-fuscae, secundariae nigrae, vexillo interno livescente; alae cauda multo minores, subtus antice livescenti-ochraceae; axillae brunnescentes; uropygium, crissum femoraque purpurea ; cauda aequalis, purpurea, apice nigro-fasciata; rostrum sanguineum, medio carinatum, triquetrum, apice dentatum, naribus vix apricis, vibrissis nigris aeque tectis; tarsi breves, longitudine fere digiti medii, flavi; digitus lateralis externus dimidius connatus. Longitudo corporis $8^{\prime}$, caudae $3 \frac{{ }^{\prime \prime}}{3}$, c. alis longioris $2 \frac{I^{\prime}}{3}$, tarsi $9^{\prime \prime}$, rostri $1^{\prime}$.

Habitat in sylvis fl. Nigri ad urbem Barcellonam, fructus lauri gigantei maturos investigans; juvenis pectore magis nigro gulaque purpureo-maculalis, adultus pectore purpureo, gula nigerrima.

\section{Genus irr. P I P R A.}

Minor, agilis, familiaris, inter frutices vociferans; rostro gracili, trigono; brevi, compressoangustato, dentato, vibrissis nullis; tarso digitis altiore; digitis gracillimis, exteriore laterali plus quam dimidio connato; cauda alisque breviusculis. 
Spegies i. PIPRa CORNUTA. Tab. Vil. F. 2.

Nigerrima; occipite cornuto, gula, jugulo femoribusque coccineis; rostro subvalido.

$D_{1}$

E s r i t t o. Corpus Pipra Rubricapilla Temm nonnihil maius, tolum nigrum, exceptis capite, gula, jugulo femoribusque purpureis; differt a Pipra Pareola modo gula non nigra sed purpurea, fronte non aurantio - sed purpureo relucenti, occipite cornuto rostroque non tenui sed crasso ac elevato.

Habitat in sylvis fl. A mazonum.

\section{SPEC1ES 2. PIPRA CORONATA. Tab. Vir. F. 1.}

Minor, aterrima; capite supra coeruleo.

D

S Cr ґт то. Corpus exiguum, supra et subtus nigrum; caput supra coeruleo relucens, plumulis basi nigris, ante, pone et infra oculos nigrum; remiges nigrae, apice vix fuscescentes; cauda alas nonnihil superans; rostrum breve, compressum, tenue, fere rectum, dentatum, supra nigricans, subtus albescens; pedes graciles, sanguineo-fuscescentes. Longitudo corporis $3 \frac{\frac{1}{2}^{\prime}}{\text {, caudae }} 1 \frac{\mathrm{I}^{\prime}}{3}$, c. alas exced. $8^{\prime \prime}$, tarsi $6^{\prime \prime}$, rostri $5^{\prime \prime}$.

Habitat ad pagum St. Pauli in sylvis fl. Solimoëns.

$$
\text { Stecies 3: PIPRA CAUDATA. Tab. VI. M. I. F.2. }
$$

Majuscula, mas coeruleus, foemina viridis; rectricibus 2 intermediis nonnihil elongatis; vertice purpureo.

$\mathrm{D}$ Scriptro. Corpus magnitudine Fringillae domesticae, supra et subtus in mare coeruleum, in foemina viride, vertice in utrisque purpureo, rostro pedibusque sanguineis.

Mas: caput supra purpureum; occiput, ante et pone oculos, gula jugulumque nigra; collum, dorsum, uropygium, rectrices intermediae, pectus abdomenque coerulea; alae supra et subtus nigrae, remigibus apice vix fuscescentibus; cauda nigra, rectricibus 2 intermediis supra coeruleis, apice subtusque nigricantibus; crissum femoraque nigricantia.

Fo em in a: frons purpurascens, reliquum corpus herbaceo-viride; remigibus olivaceo-viridibus, scapis fuscescentibus; cauda supra viridi, subtus nigricante; femoribus fusco-viridibus. 
Longitudo corporis foeminae $5 \frac{{ }^{\prime}}{2}$, caudae $2 \frac{1}{2}^{\prime}$, partis rectricum intermediarum caudam excedentis $4^{\prime \prime}$, tarsi $9^{\prime \prime}$, rostri $5^{\prime \prime}$.

Habitat in sylvis fl. Soli mo ën s, mas foemina nonnihil minor; differt a Pipra Pareola magnitudine maiore, fronte, collo subtus, pectore abdomineque non nigris, rectricibus 2 intermediis elongatis, pedibus non flavis sed sanguineis.

\section{Species 4. PIPRA FILICAUDA. Tab. Vili. M. 1. F. 2.}

Mediocris, subtus citrina, mas supra niger, capite nuchaque purpureis; foemina viridis; rectricibus elongatis, filosis.

D

ESCR I Trio. Corpus praecedente paulo minus, subtus totum citrinum. Mas: caput supra nuchaque purpurea, plumis basi flaris; frons, regio ante, pone et infra oculos citrinae; dorsum, uropygium, crissum, femora, cauda alaeque nigra; remiges se_ cundariae vexillo interno medio albae; axillae alarum flavae; alae subtus albicantes; cauda dimidia et basi, praecipue in vexillis internis plumosa, dimidia apiceque rectricum utrinque 4 exteriorum filoso-setosa, filis sive scapis elongatis introrsum nonnihil curvis, rectricibus 3 utrinque intermediis plumosis, brevibus; rostrum trigonum, nigricans; pedes graciles, sanguinei. Longitudo corporis $5^{\prime}$, caudae absque seta $1 \frac{1}{2}^{\prime}$, cum seta $3 \frac{1}{4}^{\prime}$, tarsi $7^{\prime \prime}$, rostri $5^{\prime \prime}$.

Foemina mare vix maior, supra viridis, subtus pallide flavicans; caput et frons viridia; remiges fuscae, viridi limbatae, vexillis internis non albis,

Juvenis viridis supra, subtus et fronte citrina, nucha capiteque vix purpurascentibus.

Habitat ad pagum St. Pauli in sylvis fl. Solimoëns. species 5: PIPRA HERBACEA. Tab. VIIIa. F. i:

Mediocris, tenuirostris, herbaceo-viridi relucens; abdomine sulphurescenti.

$D_{\text {s }}$ ESCripto. Corpus exiguum, aeruginoso-, vel saturate coeruleo-viride, plumis basi nigris; caput, nucha, dorsum tectricesque alarum coerulescenti-viridia; gula pectusque aeque viridia, sulphureo hinc inde maculata; abdomen crissumque sulphurescentia; femora flavo-cinerea; remiges, nec non cauda brevis fuscae, lateraliter viridi marginatae, vexillo interno medio albescenti; axillae flavicantes; cauda alis nonnihil brevior, vires- 
cens, supra apice coeruleo-fuscescens, subtus fusca ; rostrum tenue, apice acutum, dentatum, supra nigrum, subtus pallescens; pedes graciles, haud albi, sanguineo-fusci; digitus lateralis externus basi connatus; oculi brunnei. Longitudo corporis $3^{\prime}$, caudae $\mathbf{1}^{\prime}$, c. alis longioris $6 "$, rostri $5 \frac{\mathrm{I}}{2}{ }^{\prime \prime}, \operatorname{tarsi} 5 \frac{\mathrm{I}}{2}{ }^{\prime \prime}$.

Habitat in sylvis fl. A mazonum.

$$
\text { Species 6. }+ \text { PIPRA ELATA. Tab. VIII. F. z. }
$$

Minor, olivaceo-virescens, subtus viridi sulphurescens; capite nigricante, in medio aurantio; tectricibus ala rum flavo terminatis.

D E S c r i т т о. Corpus Sylvia Regulo vix maius, supra olivaceo-viride, subtus sulphurescens; caput nigricans, vertice aurantio; subcristato; gula cinerascens; tectrices alarum mediae flavo terminatae; remiges secundariae lateraliter flavo-marginatae, primariae nigro-fuscae; cauda fusca, rix viridi marginata, apice albo terminata; rostrum breve, depressiusculum, nigrum; oculi flavi; pedes breves, graciles, nigri; digitus externus lateralis basi connatus. Longitudo corporis $3 \frac{1}{2}^{\prime}$, tarsi $6^{\prime \prime}$, rostri $5^{\prime \prime}$, caudae $1 \frac{\mathrm{r}^{\prime}}{4}$, caudae alis longioris $9^{\prime \prime}$.

Habitat in sylvis Parae. An Sylvia elata et cristata Lath. et Buff.?

\section{Familia: Muscic a p a}

Platyrostres, insectivorae, solitariae, frequenter taciturnae, arboribus haud altis insidentes, brevivolantes; rostro basi planiore quam alto, vix compresso, vibrissis longis munito, apice trigono, dentato, uncinato; naribus basalibus, ovatis, membrana tectis; tarso digitis altiore; digitis lateralibus ex. ternis basi vix connatis, posticis longiusculis,

\section{Genus I. T $\mathrm{O}$ D U S.}

Rostro longiore, supra et subtus ad apicem fere aeque plano latoque; pedibus gracilibus; digito laterali externo basi connato; cauda alisque brevibus, Piprae similibus. 


\section{Species x. TODUS IMELANOCEPHALUS. Taв. IX. F. 2.}

Minor, olivaceo-cinereus, subtus flavus; fronte caudaque nigris; rectricibus apice albo-fimbriatis, exteriore extrorsum albo-, remigibus flavo-marginatis.

D

SCR IP 10. Corpus exiguum, supra cinerascens, olivaceo-viridi vix indutum, subtus laete flavum; cáput supra, praecipue frons, ante ac pone oculos nigra, supra nares flavo submaculata; nucha plumbea; dorsum cinereum, vix olivaceo virescens, plumis basi nigris; uropygium, flavicans; tectrices alarum nigrae, flavo marginatae; remiges nigrae, flavo extrorsum limbatae, apice fuscescentes, vexillo interno basali albo-fimbriato; alae subtus antice flarae; cauda alis longior, nigra, apice flavo-albo limbata, rectrice exteriore apice et margine externo flavo-alba; gula, abdomen crissumque lutea; rostrum nigrum, apice subtus modo albicans; pedes nigri. Longitudo corporis $3 \frac{{ }^{\prime \prime}}{2}$, caudae $1 \frac{1}{3}^{\prime}$, rostri $7^{\prime \prime}$, tarsi $8^{\prime \prime}$.

Habitat ad litora fl. A mazonum; differt a Todo cinereo Lath. (Buf. Pl. 585. F. 3.) capite dorsoque non viridibus, gula non alba.

\section{Species 2. TODUS CINEREUS. Tав. X. M. 1. F. 2.}

Minor, virescens, subtus flavicans; capite plumbeo; gula juguloque albis, nigro striatis; cauda nigro-fusca; rectricibus albis nullis.

$D$ E SC в 1 ту о. Corpus magnitudine praecedentis, supra laete virescens, subtus sulphureum; caput cinereo-plumbeum, ante et pone oculos griseo variegatum; dorsum uropygiumque viridia; tectrices alarum nigricantes, viridi-flavo marginatae; remiges aeque nigricantes, margine externo viridi-marginatae, margine interno basali albescente; cauda alis longior, nigricans, viridi marginata, apice albo-limbata; gula jugulumque alba, longitudinaliter nigro striata; pectus, abdomen crissumque sulphurea; rostrum nigrum, nonnihil largius quam in specie praecedente, subtus pone albescens; pedes fusci. Longitudo corporis $3 \frac{1}{2}^{\prime}$, caudae $1 \frac{1}{3}^{\prime}$, c. alis $1.10^{\prime \prime}$, tarsi $8^{\prime \prime}$, rostri $7^{\prime \prime}$.

Habitat in Brasilia; an Foemina speciei praecedentis? Todo cinereo Lath. et Buff Pl. 585. F. 3. licet simillima, differt tamen capite non viridi, gula juguloque nigro striatis, rectricibus albis nullis. Tabula nostra (X.F. 1.) exhibet avem Todo cinereo (F.2.) omnino similem ideoque foeminam eiusdem speciei a nobis receptam; discrepat vero ab illa rostro tarsisque multo brevioribus, gula non striata abdomineque pallide flavo; an species diversa? 


\section{Genus 2. PLAT YRHYNCHUS.}

Rostro trigono, supra subtusque plano, latiore quam longiore, praecipue maxilla infetiore plana, lata.

Speqes 1. PLATYRHYNCHUS XANTHOPYGUS. Taв. IX. F. 1.

Brunnescens; collo-subtus rufo; uropygio abdomineque stramineo-ochraceis; cauda nigra.

D Escriptro. Corpus Luscinia minus, supra cinereo-brunneum; caput fuscobrunneum, vertice medio basi stramineo; vibrissae mastacales confertae, nigrae, antrorsum versae rostroque haud breviores; dorsum brunneo-cinereum; uropygium, abdomen crissumque ochracea sive straminea; jugulum, pectus femoraque rufa; tectrices alarum fusco-brunneae; remiges nigro-fuscescentes; alae subtus antice stramineae; cauda alis multo longior, nigra; rostrum trigonum, longius quam latius, supra vix elevatum, nigrum, subtus planum, ochraceum, naribus vibrissis obtectis; pedes digitique graciles, fuscescentes. Longitudo corporis $4 \frac{1^{\prime}}{3}$, caudae $2^{\prime}$, c. alis 1. $1 \frac{1^{\prime}}{3}$, tarsi $7 \frac{t^{\prime \prime}}{2}$, rostri $5^{\prime \prime}$.

\section{Species 2. PLATYRHYNCHUS RUFICAUDA. Tab. XI. F. 1.}

Majusculus, fusco-olivaceus, alis caudaque rufis.

D ESCR1Ptio. Corpus magnitudine Fringillae domesticae, supra fusco-olivaceum, subtus flavo-viride; caput olivaceo-viride; dorsum viride, medio obfuscatum; uropygium rufum; gula flavo-cinerascens; pectus flavo-virescens, nebulatum; abdomen sulphurescens; femora brunnea; crissum ferrugineum; tectrices humerales obsure virescentes, reliquae rufae, medio nigro-maculatae; remiges nigro-fuscae, margine utroquerufomarginatae; alae subtus antice stramineae; cauda alis duplo maior, rufa, aequalis ; rostrum capite brevius, nigrum, planum, apice acutum, aduncum; oculi grisei; pedes graciles, fuscescentes, argenteo induti. Longitudo corporis $5 \frac{{ }^{\prime}}{2}$, caudae $2 \frac{3}{4}^{\prime}$, rostri $7^{\prime \prime}$, tarsi $7^{\prime \prime}$.

Habitat in sylvis fl. Amazonum. 


\section{Speres 3. PLATYRHYNGHUS CHRYSOCEPS. Taв. XI. F. 2.}

Mediocris, brunneus, subtus albescens; vertice basi aurantio; tectricibus alarum rufo maculatis.

$\mathrm{D}$ ESCRIprio. Corpus praecedente duplo fere minus, supra brunneum, subtus stramineo-albescens; caput brunneum, vertice fusco maculato, sublus aurantio, plumis erectilibus; genae subochraceae; gula, pectus, abdomen crissumque stramineo alba, plumis basi nigris; femora ferrugineo-brunnea; tectrices alarum brunneo-fuscae, mediae fulvo terminatae; remiges nigro-fuscae, secundariae extrorsum, praecipue introrsum basi fulvo-limbatae; alae subtus antice ochraceae; cauda alis longior, nigro-brunneo fusca; rostrum capite brevius, trigonum, apice acutum, aduncum, supra brunneo-nigrum, subtus albescens; vibrissae mastacales nigrae, porrectae; pedes altiores, graciles, brunneo-fusci. Longitudo corporis $4 \frac{1}{2}^{\prime}$, caudae $2 \frac{1}{3}^{\prime \prime}$, c. alas exced. $1^{\prime}$, rostri $5^{\prime \prime}$, tarsi $7^{\prime \prime}$.

\section{Speres 4. PLiATYRHYNCHUS SULPHURESCENS.}

TA B. XII. M. 1. F. 2 (?).

Mediocris, sulphureo-virescens, subtus viridi-flavescens; rostro plano, lato; tectricibus alarum flavo-, remigibus rectricibusque nigro - fuscis, viridi - marginatis.

$\mathrm{D}$ S C r I t т o. corpus magnitudine Fringillae domesticae, supra in mare (?) laete, in foemina pallidius olivaceo-viride, sublus viridi-sulphureum; caput viridi-nigricans, supra aures flavescens; gula albo-cinerea; pectus, abdomen crissumque viridi-flara; femora viridi-fusca; tectrices alarum virides, flavo-terminatae; remiges rectricesque nigro-fuscae, viridi-marginatae, vexillo remigum secundariarum interno medio albcflavicante; alae anticae subtus flavae; cauda alis duplo longior, aequalis; rostrum basi latum, planum, apice trigonum, aduncum, medio subcarinatum, supra nigrum, subtus planum, album; vibrissae mastacales porrectae; oculi brunnei; pedes subgraciles, argentei, digito externo laterali dimidio connato. Longitudo corporis foeminae $5 \frac{3}{4}$, caudae $2 \frac{x^{\prime}}{2}$, c. alas exsup. $1 \frac{\pi^{\prime}}{2}$, rostri $9^{\prime \prime}$; latitudo rostri $6 "$; altitudo tarsi $7^{\prime \prime}$.

Habitat in sylvis Provinciae Rio de Janeiro, Piauhy et fl. A mazonum; mas hic descriptus foemina minor differt ab illa, a cel. $\mathrm{T}$ e $\mathrm{m} \mathrm{m}$ in $\mathrm{k}$ i o vivaciter depicta et Platyrh. olivaceus nominata (Pl. 12. F. 1.) rostro minus latiore magisque attenuato, colore viridi multo vivaciore, subtus minus cinerascente tectricibusque alarum non fulvo sed flavo terminatis; an species diversae? 
Species 5. PLATYRHYNCHUS HIRUNDINACEUS. Tab. XIII. F. ..

Majusculus, brunneo-niger, subtus castaneus; alis caudaque mediis castaneis; tarsis abbreviatis; alis elongatis, cruciatis.

Descripто. Corpus magnitudine Hirundinis urbicae, supra nigro-brunneum, subtus totum castaneutn, plumis basi cinereo-nigris; caput castaneo-fuscum; uropygium crissumque castanea; tectrices alarum - brunneo - nigrae, obsure castaneo-marginatae; remiges elongatae, cauda vix minores, castaneae, apice et vexillo externo nigricantibus, vexillo remigis extimae externo rufo-marginato, secundariae castaneae, versus apicem nigricantes; alae subtus castaneae; cauda castanea, versus apicem large nigrofusco fasciata, aequalis, vexillo rectricis externae nigricante; rostrum capite vix brevius, basi largum, medio vix elevatum, apice trigonum, aduncum, supra subtusque nigrum; vibrissae mastacales breves; tarsi abbreviati, brunneo-fusci; digiti fere toti fissi, Longitudo corporis $7^{\prime}$, caudae $3^{\prime}$, c. alis 1 . $8^{\prime \prime}$, rostri $9^{\prime \prime}$, tarsi $6^{\prime \prime}$; latitudo rostri $4 \frac{1}{4} "$.

Vitam degit in interiore Brasilia, mas rostro nonnihil angustiore, coloreque supra minus obscuro.

\section{SPECIES 6. PLATYRHYNCHUS CINEREUS. Taв. XIII. F. 2.}

Minor, immaculate nigro-fuscus. subtus plumbescens; crisso rostroque subtus albis.

$\mathrm{D}$

Escriptio. Corpus Fringilla domestica minus, supra brunneo vel fuliginoso-nigricans, subtus plumbescens; caput nigro-fuscum; gula albo-cinerea; abdomen posterius crissumque albo-fulvescentia; tectrices alarum fuliginoso-fuscae; remiges primariae nigricantes, secundariae apice pallide albo marginatae; cauda alis longior, aequalis, nigro-fusca; rostrum capite brevius, basi latum, apice acutum, subaduncum, supra nigricans, subtus planum, fulvescens; pedes subgraciles, breviusculi, nigricantes. Longitudo corporis $5^{\prime}$, caudae $2 \frac{1}{2}^{\prime}$, c. alas superantis $1 \frac{1}{3}^{\prime}$, rostri $6^{\prime \prime}$, tarsi $4 \frac{\mathrm{I}^{\prime \prime}}{4}$.

Habitat in sylvis fl. Am a z on um; differt a Muscicapa caesia Temm. rostro non angusto, corpore non toto plumbeo. 


\section{SPECIES 7. PLATYRHYNCHUS FILICAUDA. Taв. XIV.}

Mediocris, nigerrimus; capite supra cinereo-albo; fronte uropygioque albis; rectricibus 2 intermediis elongatis; rostro breviusculo.

$\mathrm{D}$ ESCR IPTio. Corpus gracile, totum nigrum absque splendore chalybeo; caput supra cinereo-album, in occipite, ante et pone oculos nigrum; frons uropygiumque alba; gula nigra; remiges nigricantes, apice in foemina brunneo-fuscae, secundariae vexillo interno basali obscure albicantes; cauda alis longior, nigerrima, in foemina brunneonigra, aequalis exceptis rectricibus 2 intermediis elongatis, corpore fere longioribus, medio altenuatis, vix plumosis, versus apicem latiusculis plumosis; rostrum minutum nigrum, basi latiusculum, supra vix eleratum, vibrissis nigris; pedes nigri; digitus externus lateralis basi subconnatus. Longitudo corporis $8 \frac{3}{4}{ }^{\prime}$, caudae absque rectricibus intermediis $2 \frac{1}{2}^{\prime}$, rectricum intermediarum $6^{\prime}$, tarsi $6^{\prime \prime}$, rostri $4^{\prime \prime}$.

Habitat in Provincia Rio de Ian ei ro ad pagum Ma n i o c a, cacumini arborum minus altarum insidens; differt a $\mathrm{P}$ i p ra Leucocilla magnitudine maiore, capite non toto albo, colore corporis nigro non violaceo.

\section{Stecies 8. PLATYRHYNCHUS FLAVIVENTER. Tab. XV. F. .}

Minor, supra olivaceo-viridis, subtus luteus; capite non cinereo, supra oculos non albo-striato; tectricibus alarum flavo-viridi terminatis.

$\mathrm{D}$ ESCR IF T1 0. Corpus exiguum supra lacte olivaceo-viride, subtus tolum luteum, plumis basi cinereis; caput supra viride, ante et post oculos flavicans:; tectrices alarum minores nigricantes, viridi-luteo terminatae; remiges breves, fusco-nigrae, margine externo viridifimbriato, secundariae extrorsum flavo marginatae, basi flavae; cauda alis brevior, aequalis, brunnescenti-fusca, viridi marginatà ; rostrum breve, nigricans, supra vix carinatum, naribus fere intermediis, a vibrissis nigris remotis; pedes nigri, argenteo induti; Longitudo corporis $4^{\prime}$, caudae $1 \frac{3{ }^{\prime}}{4}$, c. alis 1 . $1 \frac{1^{\prime}}{4}$, rostri $5^{\prime \prime}$, tarsi $6^{\prime \prime}$.

Habitat in Provincia Rio de Jan e ir o; differt a Muscicapa Diope Tem. Pl. 144. F. 8. corpore subtus non cinereo, a Muscicapa eximia capite non cinereo, stria super oculos et in genis non alba. 
Siectes 9. PLATYRHYNCHUS BREVIROSTRIS. Tав. XV. 2.

Minor, supra subolivaceus, subtus lutescens; striis 2 super alas transversis, albis; periophthalmiis allis; gula albicante; remigibus rectricibusque fuscis.

$\mathrm{D}$

E S C R I T т o. Corpus exiguum, supra olivaceo virescens, subtus pallide lutescens; caput supra viride, plumis verlicis fusco alboque terminatis; stria a genis super oculós alba; gula jugulumque, sordide albicantia; tectrices alarum humerales virides, subjacentes nigro-fuscae, albo terminatae; remiges nigro - fuscae, vexillo externo viridi-marginato, introrsum et basi subflavicantes, secundariae apice albo fimbriatae; alae subtus antice flavae; cauda brunneo-fuscá, lateraliter viridi submarginata, aequalis, alis longior; rostrum nigricans, minutum, supra subelevatum; tarsi breves, nigri; digitus externus lateralis basi connatus. Longitudo corporis $4^{\prime}$, caudae $2^{\prime}$, p. c. alis 1. 1. $\frac{11}{5}$, tarsi $6^{\prime \prime}$, rostri $3 \frac{I^{\prime \prime}}{2}$.

Habitat in Provinc:a Rio de $\mathrm{J}$ aneiro; foemina hic non depicta et illi ceylonensi Swainson Pl. 3 fere similis corpore maiore, rostro paulo longiore et planiore tarsoque magis alto est. Differt a Muscicapa exinia Tem. pl. 144. F. 2. colore non laete viridi, capite non cario, gulaque non flava.

\section{Speres 10. PLATYRHYNCHUS PAGANUS. Taв. Xvi. ı.}

Majusculus, supra cinereo-olivaceus, subtus pallide flavicans; jugulo pectoreque cinereo-albis; vertice haud flavo; palpebris albis; tectricibus alarum albescenti terminatis.

$\mathbb{D}$

ESCR IPT I O. Corpus Fringilla domestica vix minus, supra cinereo-olivaceum; caput olivace-fuscum, vertice basi haud flavo et vix albo striato, periophthalmiis albis; gula, jugulum pectusque albo - cinerea; abdomen, femora crissumque sulphurescentia; striae alarum 2 vel 3 transversae, albicantes, tectricibus nigro-fuscis, albo terminatis; remiges nigro-fuscae, margine externo vix virescenti ${ }_{i}$ alae subtus antice flavicantes; cauda nigro - fusca, alis longior, aequalis, rectricibus, extrorsum viridi vix marginatis; rostrum breviusculum, trigonum, subaduncum, supra brunnescens, subcarinatum, subtus flavescens; pedes subrobusti, nigri, digitis fere totis fissis. Longitudo corporis foeminae $5 \frac{{ }^{\prime}}{2}$, caudae $2 \frac{1}{2}^{\prime}$, p. c. alis l. $1 \frac{1}{2}^{\prime}$, rostri $5^{\prime \prime}$, tarsi $9^{\prime \prime}$.

Reperitur in sylvis Rio d e Jan eiro, mas paulo minor, abdomine magis sulphurescente. 


\section{Species 2 . PLATYRHYNCHUS MURINUS. Tab. XVI. 2.}

Minor, supra brunnescens, ante et super oculos albo notatus, subtus sulphurescens, gula albescente.

D

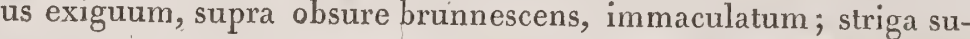
per oculos versus genas, duaeque aliae transversae super tectrices alarum flavo-albae; gula albescens; pectus, abdomen crissumque sulphurescentia; tectrices alarum fuscobrunneae, flavo-albo terminatae; remiges fusco - brunneae, vexillo interno basi flavicante; cauda alis longior, aequalis fusco-brunnescens; rostrum breve, trigonum, subelevatum, fuscescens, subtus flavescens; pedes graciles, fuscescentes. Longitudo corporis $3 \frac{3}{4}^{\prime}$, caudae $1^{\frac{3}{4}}$, p. c. alis $1.1^{\prime}$, tarsi $7^{\prime \prime}$, rostri $5^{\prime \prime}$.

\section{Genus 3. II U S C I C A P A.}

Solitaria, Platyrhyncho simillima, rostro vero longiore quam latiore, magisque cylindrico ac carinato, vibrissis munito, mandibula inferiore compress; ; tarsis plerumque elevatis, robustis.

\section{Species 1. MUSCICAPA LONGICAUDA. Tab. XVII.}

Maior, longissime caudata, supra subtusque cinerea; alis medio rufis caudaque gradata nigricantibus; collari gulam albam ambiente sanguineo-brunneo; remige rectriceque exteriore albo marginatis.

$\mathrm{D}$

Es criptio. Corpus robustum, Merula vix minus, supra subtusque cinereo canescens; caput albo-cinereum, supra oculos et in fronte albescens, plumis squamosis, medio nigro-linealis; gula, crissum nec non abdomen alba; collare jugulare usque ad aures fusco - sanguineum; dorsum, femora, uropygium pectusque cinereo plumbescentia, scapis plumarum interdum nigris; tectrices alarum remigesque secundariae nigricantes, primariae introrsum et basi flavicantes, extrorsum medio ferrugineae, apice nigricantes, albescenti vix terminatae, vexillo remigis extimae externo tenui, flavo; alae subtus ad axillas humerosque albae, medio flavae; cauda corpore duplo longior, gradata, basi brunneonigra, reliqua nigra, rectricibus 3 utrinque exterioribus longioribus, extima longissima, in vexillo externo basi flavo-albo marginata, apice largius plumosa, extrorsum subarcuata; rostrum validum, nigricans, aduncum; pedes nigri, robusti; digitus lateralis externus fere non connatus. Longitudo corporis $1^{\circ} 2 \frac{I}{2}^{\prime}$, rectricis longissimae $10 \frac{1}{3}^{\prime}$, rostri $9^{\prime \prime}$, tarsi $13^{\prime \prime}$.

Habitat in pratis paludosis Provinciae St. Pauli supra arbusta, Tezoira do Campo appellata. 
Species. 2. +MUSCiCAPA VETULA. Tab. XviII.

IMaior, supra subtusque plumbea; alis caudaque nigris; cauda haud elongata, gradata, furcata, rectrice extima extrorsum allomarginata.

$\mathrm{D}$ ESG R P I o. Corpus robustum, praecedente paulo minus, supra subtusque plumbeum; caput, collum, dorsum, pectus, abdomen, uropygium crissumque plumbea; gula vix albescens; femora brunneo-fusca; alae immaculate nigrae, subtus antice albescentes; cauda aeque nigra, alis longior, apice gradata ac furcata, vexillo rectricis extimae externo tenui, albo, apice nigro; rostrum nigrum, aduncum; pedes vix robusti, nigri. Longitudo corporis 8', caudae 4', rostri 8", tarsi 9".

Victitat in campis Provinciae St. Pauli.

Spezes Z. TMUSCICAPA FURGATA. Tab. XIX.

Maior, supra obsure olivacea, subtus citrina; vertice aurantio-rubro; rostro valido, planiusculo; cauda furcata; gula cinereo-alba; tarsis brevibus.

\section{D} ESCR I ті To. Corpus robustum, supra dorsum obsure olivascens, subtus citrinoflavum; caput nuchaque plumbea, vertice medio aurantio-rubro; gula jugulumque cinereoalba; pectus viridi-flavum; abdomen, femora crissumque citrina; tectrices alarum nigrofuscae, sordide albo terminatae; remiges aeque nigro-fuscae, basi subtus albo-livescentes; alae antice subtus citrinae; cauda alis maior, nigro-fusca, subtus plumbeo-fusca, apice subfurcata; rostrum capite fere maius, rotundato-planiusculum, nigrum, maxilla inferiore haud plana; oculi grisei; tarsi breves, nigri, digitis fissis. Longitudo corporis $8^{\prime}$, caudae $3 \frac{1}{2}^{\prime}, 1$. p. furcatae $1^{\prime}$, rostri $8^{\prime \prime}$, tarsi $8^{\prime \prime}$.

Habitat in locis campestribus Brasiliae sub nomine vago, Pendivim ${ }^{\star 6}$ mas a foemina vix diversus. 


\section{Speres 4. MUSCICAPA SULPHUREA. Taв. XX.}

Maior, supra olivaceo-virescens, subtus citrina; vertice aurantio-flavo; cauda aequali, non furcata; gula juguloqne albis.

D

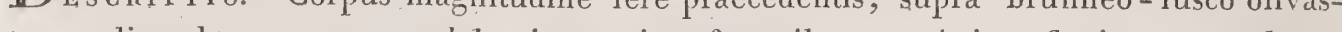
cens, alis subtus, pectore, abdomine, crisso femoribusque citrino-flavis; caput obsure cinerascens, vertice medio introrsum aurantio-flavo; gula, jugulum pectusque anterius alba; dorsum uropygiumque brunneo-fusca, olivaceo-viridi induta; tectrices remigesque alarum füsco-brunneae, virescenti marginatae, vexillo remigum interno medio pallide flavicante; cauda alis longior, aequalis, brunneo - fusca supra, subtus cinerascens, rectricibus viridi submarginatis; rostrum nigrum, trigonum, carinalum, aduncum; pedes robustiores, nigri, haud alti. Longitudo corporis $8^{\prime}$, caudae $3 \frac{{ }^{\prime \prime}}{4}$, rostri 9", tarsi $9{ }^{\prime \prime}$.

Habitat in Brasilia; differt a Muscicapa furcata cauda non furcata, rostro haud plano, vertice non rubro, a Muscicapa Joazeiro verlice non rubro, gula juguloque non albis, rostro haud tenui tarsisque non altis.

\section{Stecies 5. MUSCICAPA CINERASCENS. Taв. XXI. M. 2. Foem. 1.}

Maior, supra, praecipue subtus non plumbescens sed cinerascens; alis caudaque fuscis; rostro robusto, adunco; mare et foemina unicoloribus.

D ES CR 1 P T10. Corpus praecedente paulo minus, supra cinerascens, subtus albescenti cinerascens; caput cinereum, fusco maculatum, periophthalmiis nec albostriatis, verticeque nec flavo nec rubro; dorsum fusco cinerascens, vix olivaceo-nebulatum; tectrices remigesque alarum nigro - fuscae, in foemina rufo marginatae; alae subtus rufo-albescentes; gula, pectusque sulphurescenti-cinerea; abdomen cinereo canescens; crissum rufescens; cauda magnitudine corporis, aequalis, fuscescens, in foemina rufo marginata; rostrum crassum, basi latiusculum, nigrum, naribus vibrissis tectis; tarsi minus alti, nigri, subrobusti. Longitudo corporis $7^{\prime}$, caudae 3, , c. alis longioris $2^{\prime}$, tarsi $8^{\prime \prime}$, rostri $8^{\prime \prime}$.

Habitat in locis apricis Rio de Janeiro, foemina mare maior, remigibus rectricibusque rufo marginatis, rostro minus adunco, tarsis minus altis; differt a IMuscicapa (Tamnophilo) caesia Temm. corpore fere duplo maiore, non plumbeo sed cinereo, rostro non cylindrico, tamnophiloideo, caudaque non brevi, ab Ampelide cinerea magnitudine fere triplo minore. 


\section{Lihr. 1820 \\ Species in. HMUSCICAPA VELATA. Tá. XXI.}

Maior, supra brunnescenti-fusca, subtus et in fronte, cauda basali medioque alarum alba; capite ca. nescenti.

D

Scriptio. Corpus praecedente maius, supra brunneo-fuscum, subtus totumalbum; caput canescens, fronte, genis, auribus striaque super oculos albis; tectrices alarum nigro-fuscae, longiores, apice albicantes; remiges nigrae, vexillo interno basali secundariarumque medio nec non apice albis; uropygium, crissum femoraque alba; cauda aequalis, alis vix maior, dimidia basalis alba, reliqua nigra; rostrum nigricans, basi subdepressum, versus apicem cylindricum, capite fere longius; pedes alti, robusti, nigri. Longitudo corporis $7^{\prime}$, caudae $3^{\prime}$, tarsi 1 $3^{\prime \prime}$, rostri $9^{\prime \prime}$.

Habitat in Provincia S t. P a uli.

Speies 12. +MUSCICAPA JOAZEIRO. Tab. XXïr.

Maior, supra flavo-olivascens, subtus sulphurea; vertice coccineo, remigibus caudaque brunnescenti-lives centibus; rostro subcylindrico.

D ESCriptro. Corpus praecedente vix minus, flavo olivascens, subtus totum sulphureum; caput totum pallide brunnescens ac olivascens, vertice erectili, basi coccineo; gula, jugulum, pectus, abdomen, femora crissumque sulphurea; dorsum flavo-brunneo olivascens; uropygium nonnihil rufescens; tectrices alarum pallide fusco brunnescentes, rufo submarginatae; remiges fusco brunnescentes, vexillo interno basali sulphureo; cauda alis maior, aequalis, colore remigum, apice vero flavicanti-albo terminata; rostrum nigricans, capite fere longius, apice subcylindricum, dentatum; pedes alti, nigri, dis gitis fissis; oculi nigri. Longitudo corporis $6 \frac{1}{3}^{\prime}$, caudae $2 \frac{3{ }^{\prime}}{4}$, c. alis longioris $1 \frac{3{ }^{\prime}}{4}$, rostri $9^{\prime \prime}$, tarsi $13^{\prime \prime}$.

Habitat in campis prope pagum Joazeiro ad flumen St. Francisci, mas a foemina vix diversus. 


\section{Speres 13. MUSCICAPA POLYGLOTTA. Tab. XXIV.}

Maior, supra cinerea, subtus, excepto pectore, cinereo - alba; striga infra aures nigra; cauda basi apiceque, et alis medio albis.

Descri p tro. Corpus magnitudine fere Merulae, supra fuliginoso-cinereum, subtus ad gulam, abdomen, crissum femoraque album; caput fusco-cinereum; striga a naribus versus oculos, aliaque infra oculos albae; stria a maxilla inferiore ad collum laterale nigra; dorsum, uropygium, pectus tectricesque humerales fuliginoso - cinerea; tectrices alarum mediae longaeque nigro - fuscae, sordide albo terminatae, tectrices alarum axillares remigesque nigerrimae, omnes versus basin et vix extus conspicue albae, secundariae apice albicantes; alae subtus albae; cauda alis paulo minor, aequalis, nigerrina, apice et basi alba, rexillo rectricis extimae albo ; rostrum capite vix brevius, trigonum, nigrum, aduncum; pedes altiusculi, nigri, digitis validis, fissis. Longitudo corporis 8 ', caudae $3 \frac{3}{4}^{\prime}$, rostri $9^{\prime \prime}$, tarsi $13^{\prime \prime}$.

Habitat in Provincia S t. Pa uli, mas foemina vix minor, pectore minus fusco, caudae apice minus albo terminato; discrepat a IMuscicapa velata capite non canescente, pectore remigibusque medio non albis, cauda basi minus large alba, striaque mandibulari non nigra.

\section{SPECIES 14. +MUSCICAPA SIMILIS. TAв. XXV.}

Mediocris, supra olivaceo-fusca, subtus sulphurea, Muscicapae cayennensi simillima, diversa vero rostro brevi, capite non nigro sed fusco, verticeque non flavo sed aurantio.

Dé с г т г. Corpus Muscicapa cajennensi fere maius, supra olivascens, subtus sulphu-

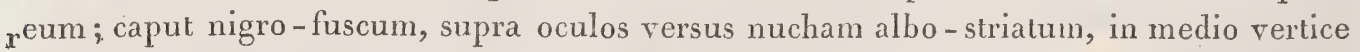
aurantium, imo coccineum, pone aures et in genis nigro-fuscum, supra nares flavo - albo notatum; gula jugulumque alba; pectus, abdomen, infra alas, crissum femoraque sulphurea; tectrices alarum brunneo-nigro fuscae, viridi-fulvo limbatae; remiges nigrofuscae, margine externo rufescente, vexillo interno praecipue versus basin rufescenti; alae subtus anlice sulphureae; cauda alis maior, aequalis, brunneo-nigro fusca, marginibus subvirescentibus; rostrum breve, carinatum, dentatum, nigrum; oculi brunnei; pedes minus alti, nigri, digitis robustis, fissis. Longitudo corporis $6^{\prime}$, caudae $2 \frac{3^{\prime}}{4}$, caudae alis longioris $1 \frac{1}{2}$, rostri $6^{\prime \prime}$, tarsi $9^{\prime \prime}$. 
Habitat ad fl. Amazonum, mas a foemina non diversus; differt a Muscicapa cayennensi, cui simillima paulo vero maior est, nonnisi rostro duplo breviore, capite haud nigro, margineque remigum externo non tam large rufo.

\section{Stectes 15. MUSCICAPA THAMINOPHILOIDES. Taв. XXVI.}

Maior, tota castanea; rostro, valido, cylindrico; abdomine ochraceo; cauda rufa.

D ESCRiptro. Corpus robustum, supra totum castaneo-rufum, immaculatum, subtus dilute castaneum; caput dorsumque castanea; gula, praecipue abdomen, femora crissumque ochraceo-ferruginea; vibrissae nigrae; tectrices alarum nigro-fuscae, castaneo marginatae; remiges fusco - nigrae, secundariae vexillo interno ochraceo, superiores rufo marginatae vel fere castaneae; alae subtus antice ferrugineae; cauda alis longior, aequalis, castaneo-rufa; rostrum validum, compressum, subcylindricum, nigrum, capite vix brevius, aduncum; pedes brunnei, albo tecti. Longitudo corporis $7 \frac{1}{2}^{\prime}$, caudae $3 \frac{1^{\prime}}{4}$, c. alis longioris 2', rostri 10", tarsi i I".

Habitat in locis sylvaticis fl. Amazonum.

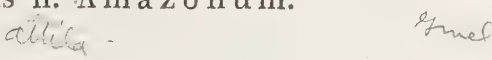

Speis 16. TMUSCICAPA CINEREA. T. T. XXVI. 2.

Maior, supra subtusque castaneo-rufa; capite nuchaque plumbescentibus; gula juguloque cinereo albicantibus; cauda rufa; rostro basi depresso, longo.

D. Escriptio. Corpus robustum, Merula minus, supra fusco-castaneum, subtus castaneo rufum, plumis basi cinereonigris; caput plumbescens nigroque striatum; nucha plumbea; gula jugulumque albicantia; abdomen rufescenti -flavo albicans; tectrices alarum nigro-fuscae, apice castaneae; remiges fusco-nigrae, extrorsum castaneo marginatae, vexillis internis basi ochraceis; secundariae superiores fere castaneae; alae subtus rufae; cauda alis longior, aequalis, rufa; rostrum capite fere longius, brunnescens, basi depressum, carinatum, apice parum elevatum, dentatum, aduncum, naribus largis ovatis, vibrissis mastacalibus nigris; pedes brunneo-fusci, alti, unguibus magnis. Longitudo corporis $7 \frac{1}{3}^{\prime}$, caudae $3^{\prime}$, c. alis longioris $1^{\prime}{ }^{\prime}$, rostri $13^{\prime \prime}$, tarsi $1^{\prime}$.

Habitat in sylvis Brasiliae, mas a foemina non diversus; an Muscicapa cinerea Lath. et Briss. haud accurate nominata? 
Species 17. MIUSCICAPA GALEATA. Tab. XXVII. Mas. XXVIII. x.Foem.

Maior, violaceo-nigra; vertice galeato; remigibus medio basique albis.

D E S C I Y т т о. Corpus Merulae simile sed dimidio fere minus, violaceo-nigrum; plumae verticis cristatae, $x^{\prime}$ longae, antrorsum curvae; alae fusco-nigrae, vexillis remigum internis medio basique albis; secundariae superiores totae nigrae; cauda alis longior, aequalis, nigra; rostrum nigrum, fere cylindricum, capite brevius; pedes robusti, alti; digiti breviusculi, fissi. Longitudo corporis $7^{\prime}$, caudae $3 \frac{3{ }^{\prime}}{4}$, c. alis longioris $1 \frac{1^{\prime}}{2}$, rostri $8^{\prime \prime}$, tarsi $10^{\prime \prime}$.

Reperitur in campis Provinciae St. Pauli, solitarie arbustis insidens; foemina (?) vel juvenis vertice non cristato, gula castanea, nigro strigilata, rostro magis compresso, gonyde breviore.

\section{Species 18. TMUSCICAPA FULVICAUDA. Tab. XXVIII. 2.}

Minor, supra fuliginoso-olivacea, subtus fulvo-alba; cauda ochracea, apice olivaceo-fusca.

Descripro. Corpus magnitudine Lusciniae, supra obscure olivascens, subtus fulvoalbicans; caput fuscum, ante oculos fulvo notatum, infra et pone oculos fulvum; gula, pectus abdomenque medio albicantia, hypochondriis fuscis; tectrices humerales olivascentes, subsequentes remigesque nigro - fuscae, extrorsum oliraceo marginatae; uropygium, crissum caudaque dimidia basalis fulvo-rufa, reliqua cauda femoraque fuscoolivacea; rostrum breviusculum, nigrum, planum, naribus vibrissis tectis; tarsi clati, fulvi. Longitudo corporis $5^{\prime}$, caudae $2 \frac{1}{5}$, c. alis $1.1 \frac{3^{\prime}}{4}$, rostri $6^{\prime \prime}$, tarsi $10^{\prime \prime}$.

\section{Species. 19. TMUSCICAPA NIVEA. тав. XXIX.}

Mediocris, tota nivea, remigibus primariis caudaeque apice nigris.

$\mathrm{D}$

ESCRIPT10. Corpus praecedente maius, supra subtusque albissimum; tectrices humerales remigesque secundariae albae, tectrices axillares remigesque primariae nigerrimae; alae subtus albae; cauda alis longior, aequalis, alba, apice nigro fasciata; rostrum breviusculum, subcylindricum, nigrum; oculi pedesque nigri, digito externo fere toto fisso. Longituda corporis $5 \frac{34^{\prime}}{}$, caudae $2 \frac{3^{\prime}}{4}$, c. alis $1.1^{\prime}$, rostri $8^{\prime \prime}$, tarsi $1^{\prime \prime \prime}$. 
Habitat solitaria in campis fl. St. Francisci prope pagum Joazeiro, ab incolis ,Tapeira, ab Azara No. 204 Irupero dicta.

Speres 20.+IMUCICAPA ALBIVENTER. Taв. XXX. Mas. x. Fo em. 2.

Minor, supra nigricans, subtus fronteque alba; cauda nigerrima, immaculata; uropygio albo.

D ESCR грт1о. Corpus praecedente minus, supra nigrum, in foemina fuliginoso-ni= gricans, subtus albissimum; caput nigrum, in fronte, super, ante, post et infra occlos album; alae breviusculae, nigrae, apice fuscescentes, subtus antice albae; cauda aequalis, tota nigra, alis longior; colli latera, gula, pectus, abdomen, femora crissumque alba; rostrum nigricans, tenue; pedes nigricantes; oculi grisei. Longitudo corporis $4 \frac{x^{\prime}}{2}$, - caudae $1 \frac{1^{\prime}}{2}$, c. alis $1.1 \frac{1}{3}^{\prime}$, rostri $7^{\prime \prime}$, tarsi $9{ }^{\prime \prime}$.

Habitat in campis Brasiliae; mas junior dorso tectricibusque humeralibus magis fuliginosis; foemina, ob similitudinem rostri Muscicapa dominicana (T.XXX. 2.) falso a mè signata, capite dorsoque fuliginoso - cinereis, alis fuliginoso-fuscis, rostro rotundatoconvexo, nigro, subtus albo.

\section{Speries $2 i . \neq$ MUSCICAPA DOMINICANA. Tä. XXIX. 2.}

Minor, nigerrima; capite colloque albis; rostro convexo, plano, nigro, subtus albo; alis apice fuscescentibus.

D E CR IP TrO. Corpus magnitudine praecedentis, totum nigrum, excepto capite, gula juguloque, raro uropygio albis; alae nigrae, in junioribus fuliginoso-fuscae; cauda aequalis, alis longior, nigra; rostrum rotundato-convexum, capite paulo brevius, supra nigricans, subtus albescens, planiusculum, apice fuscum; pedes minus alti, nigricantes; oculi grisei. Longitudo corporis $5^{\prime}$, caudae $2^{\prime}$, c. alis 1 . 1', rostri $9^{\prime \prime}$, tarsi $8^{\prime \prime}$.

Habitat in Provincia Parae sub nomine , Viuva,g. 
Mediocris, brunneo-fusca. subtus albo flavescens, Platyrhyncho pagano similis, maior vero, caudaque longiore large rufo-fimbriata, rostro longiore, crisso albo.

D longiore large rufo - fim riata, rostro longiore,

Deriotio. Corpus magnitudine Fringillae domesticae Platyrhynchoque pagano multo maius, supra fusco-brunneum, raro virescenti striatum, subtus flavo albicans, pectore fuliginoso, flavo - albo maculato; caput maris nigricans, vertice subtus large citrino-, foeminae fusco-brunneum, vertice immaculato; striga super oculos versus occiput et fronten alba; macula supra aures brunneo-fusca, stria subjacente alba; gula-albicans; abdomen, femora nec non crissum flavo-alba; tectrices alarum nigro-fuscae, humerales rufo-, reliquae albo limbatae; remiges longae nigro-fuscae, primariae extrorsum rufo-, secundariae albo-marginatae, subtus basi vexilloque interno pallide flavo-marginatae; cauda alis longior, aequalis, nigro-fusca, rectricibus rufo marginatis; rostrum longum, rectiusculum, brunneo-fuscum, maxilla inferiore non plana sed compressa, basi rufa, gonyde brevi; pedes breves, nigricantes. Longitudo corporis $5 \frac{\mathrm{I}^{\prime}}{2}$, caudae $3^{\prime}$, c. alis $1.2^{\prime}$, rostri $7^{\prime \prime}$, tarsi $7^{\prime \prime}$.

Habitat in Provincia fl. A ma z on u m, foemina vertice non flavo-maculato; differt a Platyrhyncho pagano corpore subtus minus flavicante caudaque longioribus, rostro longiore, subtus nec plano nec gonyde brevi, remigibus rectricibusque non viridi sed rufo marginatis.

Species 23. TUSCICAPA MYSTACEA. Tав. XXXIa. M. 1. Foem. 2.

Mediocris, supra subtusque alba; striga super aures, alis caudaque apice alba nigris; dorso medio albo-fuliginoso.

D

ES SR I P 10 . Corpus Luscinia minus, supra subtusque album; caput, gula, collum supra et subtus, uropygium, pectus, abdomen, dorsum et versus uropygium crissumque albissima; genae strigaque ab oculis super aures nigrae; dorsum fuliginoso-album; femora antice alba, postice brunneo-fusca; tectrices alarum remigesque nigrae; alae subtus antice vix albostrigilatae; cauda subgradata, alis longior, nigerrima, apice albo-fasciata, tectricibus supracaudalibus albis, nonnullis longioribus; rostrum nigrum, tenue, den-

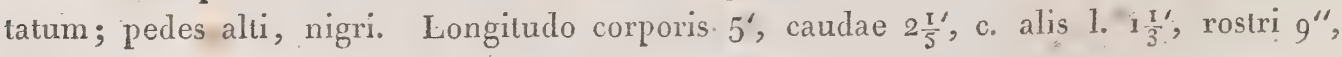
tarsi $1^{\prime}$. 

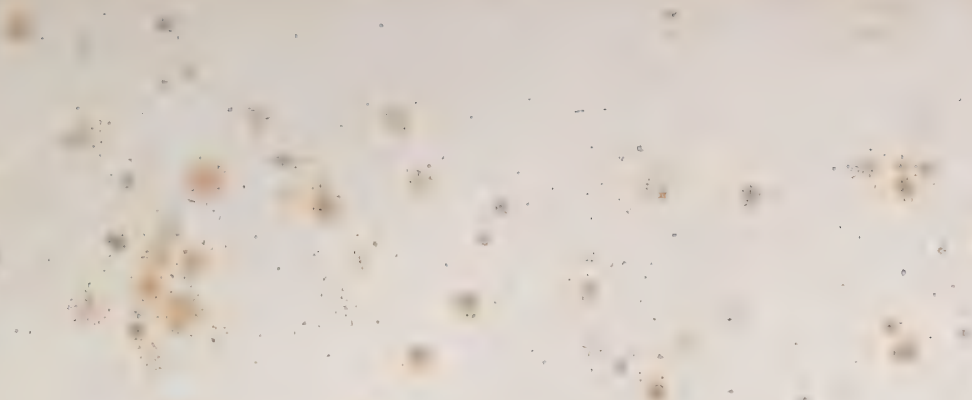

Habitat in Provincia B a hia e; foemina dorso medio remigibusque magis fuliginosis, Saxicolae auritae Temm. pl. 159 . similis, ab hac vero dissimilis patria, rostro Muscicapae proprio, caudaeque apice albofasciato.

\section{Familia: L a $n$ i i.}

Carnivori, frequenter inter arbusta saltatorii; rostro rectiusculo, acute compresso, longiusculo, dentato, versus frontem elongato; gonyde compressa, ascendente; naribus subbasalibus, subrotundis; vibrissis mastacalibus raro conspicuis; alis frequenter minoribus; tibia digito medio longiore; digito laterali externo frequenter basi connato.

\section{iGenus i. TH A M N O P H I L US.}

Brevialatus; rostro capite vix breviore, fere recto, frequenter crasso apiceque hamato; tarsis digito medio altioribus; digito postico longo; plumis corporis floccosis.

\section{SFEres 1. THAMINOPHILUS ALBIVENTER. T. XXXII. M. 1. Fo o em.2.}

Maior, subtus totus albus, mas supra nigerrimus, foemina supra cinammomea; fasciis alarum 3 allis; cauda nigra, albo fasciata.

D ES C R т т то. Corpus Merula vix minus, in mare supra nigrum, in foemina cinammomeum, in utrisque subtus albissimum; plumae capitis erectiles, acuminatae, dorsi medii basi albae, abdominis basi nigrae; gula, collum laterale, pectus, abdomen, femora crissumque alba; tectrices alarum nigrae, albo large marginatae seu albo trifasciatae, axillis albo variegatis; remiges nigrae, vexillo interno medio basique albo, externo albomarginato; cauda alis longior, fere aequalis, nigra, albo 8 fasciata, fasciis subtus permeantibus, rectricibus 2 intermediis haud raro parum albo maculatis; oculi flavorubri; rostrum robustum, nigrum, apice hamatum, naribus non tectis; pedes robusti, alti, nigri. Longitudo corporis $8^{\prime}$, caudae $3 \frac{1}{2}^{\prime}$, c. alis $1.2^{\prime}$, rostri $1^{\prime}$, tarsi $1 \frac{1}{4}^{\prime}$.

Habitat, ab Azara Legrand Batara No. 211 , a Lichtensteinio Lanius Stagurus, a Vieillot Thamnophilus maior nominatus, in sylvis fl. St. Francisci, sphegibus victitans; foemina mare vix minor, colore supra immaculate cinammomeo. 


\title{
Spéies 2. THAIMNOPHILUS LINEATUS. T.XXXIII. Mas a. Foem. 2.
}

Mediocris, supra castaneus, subtus niger, albo-fasciolatus, mas capite nigro, fuemina capite cinammomeo.

$\mathrm{D}_{\mathrm{s}}$ Ecripirio. Corpus praecedente fere duplo minus, supra castaneum, subtus totum albo nigroque fasciolatum; caput maris nigrum, vix albo punctatum, foeminae castaneum; nucha totumque collum maris foeminaeque nigra, albo variegata; dorsum castaneum, plumis floccosis, basi nigris; uropygium rufo-nigrum; apice nigro alboque-fasciatum; gula, pectus, abdomen, crissum femoraque nigro alboque fasciolata, raro fulvo nebulata; tectrices alarum remigesque castaneae; remiges apice vix nigro fuscescentes; cauda alis maior, aequalis, castanea; rostrum capite vix brevius, nigrum, apice aduncum, maxilla inferiore supra albo marginata; pedes altiusculi, argenteo induti, digito laterali externo basi connato. Longitudo corporis $5 \frac{3}{4}$, caudae $2 \frac{i^{\prime}}{2}$, c. alis $1.1 \frac{1^{\prime}}{2}$, rostri $11^{\prime \prime}$, tarsi $1^{\prime}$.

\section{SRECIES 3. THAIMNOPHILUS RADIATUS.}

\author{
Mas. Taв. XXXV. 2. Foem. TAв. XXXVIII. 1.
}

Mediocris, mas supra niger, albo undulatus, subtus albus, nigro-fasciatus, foemina supra cinammomea, subtus fulvescens, nigro transversim lineata; cauda maris albogultata capiteque nigris, foeminae cinammomeis.

\section{$\mathrm{D}$}

ESCRPT10. Corpus praecedente fere maius, maris nigrum, albo undulatum, foeminae cinammomeum, subtus fulvescens, nigro lineatum; caput maris nigrum, immaculatum, foeminae cinammomeum, in occipite, supra aures, supra, pone, infra et ante oculos albo nigroque variegatum, plumis verticis erectilibus; dorsum uropygiumque nigra, albo undulata; tectrices alarum nigrae, albo fasciatae; remiges nigrae, albo maculatae, apice vix fuscescentes ; alae subtus antice albescentes; gula loraque alba, nigro strigilata ; pectus, abdomen, crissum femoraque alba, nigro fasciata, fasciis pectoris subcordatis; cauda alis longior, aequalis, nigra, praecipue extrorsum et lateraliter albo maculata, rectricibus internis vix albo maculatis; rostrum validum, capite minus, nigro-plumbescens, apice aduncum; pedes altiores, nigri, albo induti, digitis basi connatis. Longitudo corporis 6', caudae $2 \frac{1}{2}^{\prime}$, c. alis $1.2^{\prime}$ rostri $10^{\prime \prime}$, tarsi $10^{\prime \prime}$.

- Habitat in sylvis Brasiliae, foemina supra; cauda capiteque cinammomeis, tectricibus alarum obsure nigro-undulatis, subtus fulvescens, nigro transversim lineata. 
25

SPECIE's 4. THAMINOPHILUS GUTTATUS. Taв. XXXV. .

Maior, supra niger, fulvo-guttatus, subtus fulvescenti-albus; rostro crasso; pectore nigro-maculato, alis caudaque fulvo-fasciatis.

Descrip то. Corpus praecedente paulo maius, supra nigrum, fulvo guttatum, subtus antice albicans, postice rufo-fulvum; caput nigrum, fulvo striatum vel maculatum, genis et circa oculos fulvescentibus; gula albicans; pectus albescens, nigro maculatum; abdomen, crissum femoraque rufescentia; tectrices alarum nigrae, fulvo gultatae; remi ges caudaque longa subaequalis nigrae, remiges 5-, cauda angulate ful vo $10-$ fasciatae, vexillis remigum internis basi albis; alae subtus albae; cauda subtus plumbescens; rostrum crassum, altum, aduncum, supra nigricans, subtus albescens; pedes alti, nigro-plumbei. Longitudo corporis $7 \frac{1}{3}^{\prime}$, caudae $33^{\prime \prime}$, c. alis $1.3^{\prime}$, rostri $11^{\prime \prime}$, tarsi $x^{\prime}$.

Habitat in sylvis Provinciae St. Pauli.

$-2 \sin 4+1+2=$

Species 5. THAMNOPHILUS AGILIS. Taв. XXXIV. '.

Minor, immaculate olivaceo-viridis, subtus albus; capite cinereo, supra oculos albo-striato; loris fulvis.

Dériptio. Corpus Sylviae simile, sulphurescenti-viride, subtus lacteum; caput plumbescens; striga a naribus super oculos versus occiput alba; lora crissumque viridi fulvescentia; pectus lateraliter vix virescens; gula, abdomen femoraque albicantia; tectrices humerales virescentes, axillares remigesqne fuscae, viridi marginatae; alae subtus antice flavescentes; cauda breviuscula, supra sulphureo-viridis, nec non fuscescens, subtus cinerascens; rostrum tenue, non altum, compressum, dentatum, supra brunnescens, subtus albescens; pedes breves, fusci, vix albo obtecti, digitis breviusculis. Longitudo corporis $4 \frac{x^{\prime}}{2}$, caudae $1 \frac{1}{3}^{\prime}$, c. alis $1.1^{\prime}$, rostri $7^{\prime \prime}$, tarsi $7^{\prime \prime}$.

Mas a foemina vix diversus; differt a Lanio (Thamn.) guianensi Lath. corpore multo minore, rostro non crasso et supra oculos non rufo striato.

7 


\section{SPECIES 6. THAMNOPHILUS AFFINIS. TAB, XXXIV. 2.}

Praecedente minor, viridis, subtus virescens; capite viridi; rostro tenui, brevi; tarsis sanguineis; striga super oculos alba nulla; gula abdomineque cinerascentibus.

D

escripto. Corpus exiguum, praecedenti simillimum sed minus, supra viride, subtus cinereum; caput viride, supra nares vix flavescenti maculatum, striga alba super oculos nulla; lora fusco viridia; gula jugulumque cinerea; pectus cinereum, medio viride; abdomen cinereo-virescens; alae supra virides, subtus sulphureo-virides, remigibus fuscis, extrorsum viridi marginatis; cauda supra sublusque olivaceo-viridis; rostrum breve, tenue, brunneo-albescens, naribus minutis; pedes breves, sanguinolenti, albo subinduti. . Longiludo corporis $4^{\prime}$; caudae $1^{\frac{3}{4}}$, c. alis $1.1^{\prime}$, rostri $5 \frac{1}{4}{ }^{\prime \prime}$, tarsi $8^{\prime \prime}$.

Habitat in sylvis Parae; differt a Muscicapa Diope Temm. pl. 144. f. 1 rostro non depresso sed compresso, a specie praecedente corpore minore, subtus non albo, striga alba super óculos nulla, rostro breviore, capite non cinereo.

\section{Spectes 7. THAMNOPHILUS STRIGILATUS. Taв. XXXVI. 1.}

IMaior, olivaceo-fuscus, subtus fulvescens; capite dorsoque fulvo-albo strigilatis; alis caudaque cinammomeis.

$\mathrm{D}$

escripto. Corpus Merula duplo minus, supra brunneo-olivaceo fuscescens, longitudinaliter fulvo strigilatum, subtus pallide fulvescens; tectrices alarum remigesque secundariae cinammomeae; primariae vexillo externo rufae, interno apiceque nigro - fuscae; alae basi subtusque ochraceae; cauda alis multo longior, aequalis, castaneo-rufa; abdomen posterius femoraque fuliginosa; gula, pectus abdomenque anterius ochraceofulva; crișum pallide rufescens; rostrum longum, compressum, triquetrum, aduncum, brunneo-ochraceum; pedes haud altiusculi, argentei. Longitudo corporis $6 \frac{I^{\prime}}{2}$, caudae $3^{\prime}$, c. alis $1.2^{\prime}$, rostri $9^{\prime \prime}$, tarsi $9^{\prime \prime}$. 


\section{Species 8. THAMNOPHILUS STELLARIS. Tab. XXXvi. 2.}

Mediocris, totus plumbeus; capite nigro॰, tectricibus humeri albo-guttatis; cauda brevissima; remigibus basi subtus rufis; rostro longo.

D ESCR I t I O. Corpu’̀ magnitudine Fringillae domesticae, supra plumbeum, subtús plumbeo-cinereum; caput supra nigrum, loris genisque cinereis; tectrices dorsi anterioris plumbeae, apice albo guttatae; remiges secundariae plumbeae, primariae nigro-fuscae, margine externo plumbeae, interno basique rufae; cauda brevis, vix conspicua, plumbea; rostrum capite vix brevius, validum, aduncum, supra nigricans, subtus cinerascens; pedes parum alti, nigri, argenteo obtecti. Longitudo corporis $4 \frac{T^{\prime}}{2}$, caudae $1^{\prime}$, rostri $10^{\prime \prime}$, tarsi $8^{\prime \prime}$. infumatis.

Habitat in Provincia Parae, foemina fronte magis cinerea, gula pectoreque

\section{Species. 9. THAMNOPHILUS RUFICOLLIS. Taв. XXXVII.}

Mediocris, fuliginoso-cinereus, subtus, capite colloque rufus; tectricibus alarum caudaeque apice albomarginatis.

D

ESCRIPT10. Corpus praecedente vix maius, supra cinereum, brunnescenti vix nebulatum, subtus rufum, plumis floccosis; collum, gula pectusque, praecipue caput totum rufa; femora et abdomen pallidius rufescentia; crissum nec non uropygium rufescentia; tectrices dorsi anterioris cinereae, basi vix albo maculatae, humeri brunnescentes, mediae alarum longaeque nigrae, albo fimbriatae; remiges nigro-fúscae, vexillis externis vix albo vel brunneo-, internis albo marginalis; cauda alis paulo longior, nigra, apice albo notata; rostrum validum, aduncum, supra nigricans, subtus albescens; pedes nigri, haud breves. Longitudo corporis $5 \frac{{ }^{\prime}}{2}$, caudae $2^{\prime}$, c. alis $1.1^{\prime}$, rostri $9^{\prime \prime}$, tarsi $9^{\prime \prime}$.

\section{Speres 10. THAMINOPHILUS ALBONOTATUS. T. XXXVII، 2، Mas.}

Mediocris, supra subtusque plumbescens; alis caudaque nigris; tectricibus alarum albo fimbriatis, dorsi medii basi albis; cauda apice albo-notata.

$\mathrm{D}$

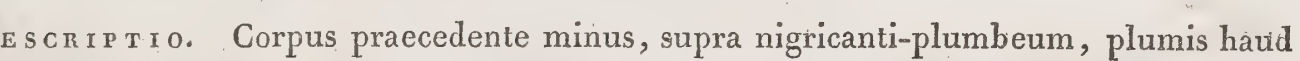
floccosis, subtus cinereo plumbescens; caput supra nigricans, super, infra poneque oculos 
cinerascens; gula, pectus, abdomen crissumque plumbeo-cinerea; femora fusca; tectrices dorsi plumbescentes, dorsi medii nigro-, basique albo-maculatae; uropygium nigrum, apice albescens; tectrices alarum humerales nigrae, albo - griseae, reliquae nigrae, apice albomaculatae, longiores albo marginatae; remiges nigrae, introrsum basi albo-marginatae; alae subtus antice albescentes; cauda nigerrima, apice albo notata; rostrum nigricans, rostro speciei praecedentis tenuius ac paulo brevius; pedes minus alti, nigri. Longitudo corporis $5^{\prime}$, caudae $2^{\prime}$, c. alis 1. 1 $\frac{{ }^{\prime}}{4}$, rostri $8^{\prime \prime}$, tarsi $8^{\prime \prime}$.

Foemina (T. XXXVIII. 2.) differt a mare hic descripto corpore supra brunneocinammomeo, subtus pallide fulvo, capite cinammomeo, remigibus cinammomeo-fuscis, extus rufo-marginatis, cauda fusco-cinammoméa, apice vix fulvo fimbriata; juvenes brunnei remigibus fuscis tectricibusque albo notatis.

Spéres 11. THAMNOPHILUS MELANOCEPS. TAB. XXXIX. 1.

Majusculus, totus castaneus; capite colloque nigris; tarsis albis; cauda breviuscula; rostro subvalido.

$\mathrm{D}_{\mathrm{E}}$ ES C 1 P 1 10. Corpus Merula paulo minus, supra subtusque rufo-castaneum, plumis dorsi floccosis, basi cinereis; caput collumque tota nigra, plumis frontis retrodecumbentibus, naribus membrana larga tectis; tectrices remigesque secundariae superjacentes castaneae, remiges reliquae breves, vexillo externo castaneo, interno nigro-fusco, basi rufescenti; cauda aequalis, cinammomea; pectus, abdomen, crissum femoraque rufocastanea; rostrum subvalidum, capite vix brevius, nigrum, dentatum; oculi nigri; pedes alti, albo-flavo obtecti. Longitudo corporis $6 \frac{1^{\prime}}{2}$, caudae $2 \frac{11}{2}$, c. alis 1.2 ', rostri $11^{\prime \prime}$, tarsi $14^{\prime \prime}$.

Habitat in sylvis Parae.

\section{SPECIES 12.4THATINOPHILUS LEUCONOTUS. Taв. XXXIX. 2.}

IMediocris, nigerrimus; nucha albo-cincta; rostro tenui, longiusculo.

D E S C R I T I O. Corpus praecedenteminus, supra subtusque nigerrimum; plumae frontis setosae, truncatae, usque ad nares membrana non tectas excurrentes, nuchales albissimae, 
apice nigrae; rostrum nigro - brunnescens, tenue, non altum, aduncum; pedes sanguinolenti, albo tecti. Longitudo corporis $6^{\prime}$, caudae $2 \frac{3}{4}$, c. alis $1.1 \frac{1}{2}^{\prime}$, rostri $9^{\prime \prime}$, tarsi $1^{\prime}$.

Habilat in sylvis $\mathrm{P}$ arae; forsan mas praecedentis, rostro tenui licet dissimilis?

\section{Stecies i3. x THAMNOPHILUS GRISEUS.}

Mas, Taв, XLI. 1, Foem. TAв. XL. 1。

Minor, supra fuscus, subtus niger, foemina rufa; rostro maxime tenui, supra oculos albo-strigilato; tectricibus alarum caudaque nigra apice albo-maculatis; alis brevibus.

D E S C R I T I o. Corpus maris exiguum, supra brunneo-fuscum vel fuliginosum, subtus nigerrimum, hypochondriis albis, floccosis; caput brunneo-fuscum, striga a naribus super oculos alba; genae loraque nigra; gula, pectus, abdomen intermedium, femora crissumque nigerrima; latera colli vix albicantia; tectrices alarum nigricantes, apice albo notatae; remiges nigricanti fuscae, vexillo interno ad basin albicanti; axillae alaeque sublus albicantes; cauda subinaequalis, nigerrima, apice albo maculata, rectricibus intermediis vix albo terminatis; rostrum nigrum, maxime tenue, apice compressiusculuin, dentatum; oculi nigri; pedes subelevati, fusci, argenteo tecti. Longitudo corporis $4 \frac{\mathrm{r}^{\prime}}{2}$, caudae $2^{\prime}$, c. alis $1.1 \frac{{ }^{\prime}}{3}$, rostri $8^{\prime \prime}$, tarsi $9^{\prime \prime}$.

Habitat, Sylvia grisea a Latha mic et forsan Myothera superciliaris a Lich te nstein i o dicta, in Prorincia Parae; foemina mare vix maior, supra cinammomea, subtus alba, tectricibus alarum nigricantibus caudaeque nigrae apice albo-notatis, remigibus brevioribus, fuscis, rufo marginalis, genis femoribusque nigricantibus.

\section{Spéres 14. THAMNOPHILUS STRIATUS. Taв. XL. 2.}

Minor, rufescens, supra fulvo striatus, subtus albescens, nigro fulvoque variegatus; rostro crassiusculo, brevi; hypochondriis rufis; gula alba.

D

SCR т то. Corpus praecedente vix maius, supra rufescens, rufo-fulvo nigroque maculatum vel variegatum, subtus album, fulvo nigroque subvariegatum; caput nigricans, fulvo-rufo maculatum; tectrices alarum rufescentes, apice fulvo-guttatae; remiges nigro-fuscae, margine externo brunneo, interno basique fulvo; hypochondria laete rufa; uropygium rufescens, floccosum; pectoris latera nigricantia, fulvo-maculata; fe- 
mora albicantia, infra brunnea; cauda breviuscula, brunneo-fusca, apice vix fulvo notata; rostrum subvalidum, triquetrum, supra nigricans, subtus albescens; pedes vix alti, argentei. Longitudo corporis $4 \frac{1}{3}^{\prime \prime}$, caudae $2 \frac{1}{2}^{\prime}$, c. alis $1.1^{\prime}$, rostri $7^{\prime \prime}$, tarsi $9^{\prime \prime}$.

\title{
Species 15. +THAMNOPHILUS GULARIS. Taв. XLI. 2.
}

Minor, supra rufescens, subtus cinereus; gula nigra, albo guttata; alis caudaque rufis; tectricibus alarum nigris, apice fulvo maculatis; rostro tenui.

D.

Escriptio. Corpus exiguum, magnitudine antecedentis, supra rufum, subtus plumbeum; caput fusco rufescens, supra oculos loraque fulvo variegatum; genae, infra oculos, gula jugulumque nigra, albo gultata; pectus plumbeum; abdomen posterius vix brunnescens; tectrices alarum nigrae, vix rufescentes, apice fulvo guttatae; remiges nigro-fuscae, margine externo rufescenti; alae subtus cinerascentes; cauda brevis, brunneo-rufa; femora fusca; rostrum tenue, nigricans, acutum; pedes graciles, vix alti, fusci. Longitudo corporis $4^{\prime}$, caudae $1^{\prime}$, c. alis $1 . \frac{1}{2}^{\prime}$, rostri 6", tarsi 8 ".

\section{SPEcies 16. +THAMNOPHILUS MIYOTHERINUS.}

\author{
Tав. XLII. IM. x. F. 2.
}

Mediocris, supra plumbeo-nigricans, subtus plumbescens; fronte strigaque super oculos albis; gula, loris genisque nigerrimis; cauda brevi, alis vix longiore.

D,

Escripto. Corpus praecedente maius, supra plumbeo-nigricans, subtus plumbescens; caput plumbescens, fronte strigaque superoculari albis velalbescentibus; genae, ante, infra et pone oculos, latera colli gulaque nigerrima; pectus, abdomen crissumque maris cinerea, foeminae plumbea; tectrices alarum plumbeae, naris parum albo terminatae, maculis albis foeminae nullis; remiges nigro-fuscae, plumbeo extrorsum marginatae; cauda aequalis, brevis, alas vix excedens, fusco-nigra; rostrum nigrum, tenue, longum, vix altum, apice dentatum; oculi brunnei; pedes alti, nigri, subgraciles; Foemina mare vix maior, fronte magis alba, corpore subtus non cinereo sed plumbeo, tectricibus non albo-maculatis. Longitudo corporis $4 \frac{1}{2}^{\prime}$, caudae $\mathbf{1}^{\frac{1}{4}}$, c. alis $1.7^{\prime \prime}$, rostri $9^{\prime \prime}$, tarsi $I^{\prime}$. 
SPECIE $17 .+$ THAMNOPHILUS MELANOGASTER. T. XLII. .

Minutus, supra et in capite plumbeus, subtus nigerrimus; canda brevissima, nigra; tarsis brevissimis; hypochondriis albis; tectricibus alarum, scapularibusque albo maculatis; rostro tenerrimo.

$\mathrm{D}$

E SCRIPrio. Corpus Thamnophilo griseo minus, supra plumbeum, subtus nigrum; caput plumbeum, striga superoculari alba nulla; gula, pectus abdomenque nigra; crissum nigro-plumbeum; hypochondria alaeque subtus albissima; tectrices scapulares subjacentes albae, reliquae alarum nigrae, albo terminatae; remiges breves, nigro-fuscae, vexillo interno basi albo; rostrum tenerrimum, nigrum, subcylindricum; tarsi nigri, breves; oculi brunnei. Longitudo corporis $3 \frac{3{ }^{\prime}}{4}$, caudae 1', c. alis 1. $7^{\prime \prime}$, rostri $7^{\prime \prime}$, tarsi 6".

Habitat in sylvis prope pagum $\mathrm{Curupa}$, propugnaculum haud insigne ad flumen A mazonum; foemina alis magis brunneo-fuscis.

\section{Familia: Fringilla e.}

Brevi-, crassi- et coni-rostres, frequenter familiares, granivorae, garrulae ac saltatoriae; rostro brevius culo, conico, basi crasso, elevato, convexo, apice plus minusve dentato, vibrissis vix tecto; naribus exiguis, subbasalibus, alis, rotundis, non membrana sed materia cornea cinctis; tarsis haud altis; alis caudaque mediocribus; digito externo laterali vix connato.

\section{GENUS I. PA CH Y R H N CH US.}

Muscicapas inter, Lanios Fringillasque intermedius, solitarie ac tranquille arboribus minus altis insidens, insectis victitans; capite crasso; rostro vix brevi, crasso, alto, rotundato-convexo, ad frontem largo, apice dentato, vibrissis mastacalibus raris; lingua apice fissa; digito laterali externo basi connato,

\section{Stecies .1. PACHYRHYNCHUS VARIEGATUS. Tas. Xlíi. 2.}

Mediocris, olivaceo-cinerascens; alis caudaque nigris; tectricibus-alarum caudaeque apice albicantibus.

$\mathrm{D}_{\mathrm{s}}$ E scripti o. Corpus Fringilla domestica paulo minus, supra olivaceo-cinerascens, hinc inde nigro-coeruleo nebulatum, subtus sordide cinereo-flavicans; caput fuliginosum, nigro-coeruleo maculatum ac renitens; dorsum posterius uropygiumque cinereoolivacea; tectrices alarum nigricantes, humerales fulvescentes, subsequentes flavo-albo terminatae; remiges fusco-nigrae, margine externo fulvescente, interno albo-flavicante; 
alae subtus antice flavo - albicantes; cauda nigerrima, apice albo notata; gula cinerascens; pectus, abdomen crissumque cinereo - sulphurescentia; femora fusca ; rostrum minus crassum, supra plumbeo-fuscum, subtus albicans, aduncum, ribrissis nigris; pedes plumbeonigri, graciles, digitis tenerrimis, longis. Longitudo corporis $5^{\prime}$, caudae $2^{\prime}$, c. alis $1.1 \frac{1^{\prime}}{3}$, rostri 8", tarsi 10". An Foemina Pachyrhynchi nigri?

\title{
Spenes 2. PACHYRHYNGHUS CAJANUS. Tab. XLIV. 1. Mas.
}

Maior, supra cinereo plumbescens, 'subtus albescens; alis caudaque tota nigerrimis; foemina supra subtusque nigro strigilata.

Décrít то. Corpus Fringilla domestica maius, supra cinereo-plumbescens, maris immaculatum, foeminae nigro strigilatum, subtus album, foeminae modo nigro strigilatum; caput maris nigrum, striga inframaxillari haud nigra, plumis basi plumbeis, ante et circa oculos nudum, foeminae plumbescens, nigro-striatum; tectrices humerales vix plumbescentes, longiores alarum plumbeo - albicantes, tectrices mediae, axillares remigesque nigerrimac, remige primaria secunda nec tenui nec falcata; alae subtus plumbescentes; cauda aequalis, tota nigerrima, albo non fasciata; dorsum uropygiumque cinereoplumbea; gula, pectus, abdomen, femora crissumque alba; rostrum maxime crassum, convexum, basi subtusque albescen's, apice nigro fasciatum; pedes robusti, nigri. Lon, gitudo corporis $7^{\prime}$, caudae $2 \frac{3}{4}^{\prime}$, c. alis 1 . $1 \frac{1}{3}^{\prime}$, rostri $1^{\prime}$, tarsi $9^{\prime \prime}$. strigilata.

Habitat in campis Provinciae Piauhy, foemina mare vix maior nigro undique

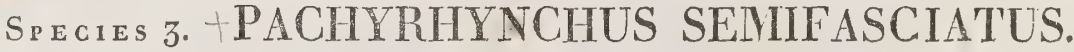

\author{
TA B. XLIV. 2. Mas.
}

Maior, supra plumbescens, subtus plumbeo albicans; occipite albicante; fronte, loris, striga inframaxillari mentoqne nigerrimis; cauda albicante, medio large nigro fasciata; remige primaria secunda breviore, falcata, tenui.

Descrixio. Corpus magnitudine praecedentis, supra immaculate plumbeum, subtus plumbescenti albicans; caput supra album, fronte, loris, striga inframaxilläri supraorbitalique nigerrimis, ante et circa oculos nudum; mentum nigrum; gula albicans; pectus, abdomen, crissum femoraque plumbescenti-albicantia; tectrices alarum humerales. 
et posteriores superjacentes plumbescentes, reliquae remigesque nigrae, secundaria secunda minore, tenui, falcata; alae subtus albescentes; cauda aequalis, albicans, medio nigro-fasciata; rostrum maxime crassum, convexum, basi subtusque carneo-albescens, apice nigro fasciatum; pedes robusti, nigri; oculi brunnei; Longitudo corporis $7^{\prime}$ caudae $3^{\prime}$, c. alis 1. $1 \frac{1}{2}^{\prime}$, rostri $1^{\prime \prime}$, tarsi $11^{\prime}$.

Habitat in Provincia P ara.

Speies 4. PACHYRHYNCHUS GUVIERI. Tar. XLV. .

Mediocris, viridis, subtus fulvescens; capite supra nigro; nucha cinerea; gula alba; pectore flavo.

D

ESCrít o. Corpus Fringilla domestica minus, supra flavo-viride, subtus fulvescens; caput supra violaceo-nigrum, lateraliter viridi vix maculatum, genis, infra poneque oculos albis, superciliis flavis; nucha cinerea; gula alba; pectus flavum; abdomen, hypochondria, femora crissumque albicanti-fulvescentia; dorsum uropygiumque viridia; tectrices alarum virides, vix nigro maculatae; remiges nigricantes, margine externo virescenti, interno flavescenti; alae subtus flavescentes; cauda haud longa, virescenti fujca, margine externo viridi, apice vix albicante; rostrum minus crassum, nigrum, subtus plumbescens; pedes graciles, plumbeo-fusci. Longitudo corporis $5^{\prime}$, caudae $1_{3}{ }^{\prime \prime}$, c. alis 1. 9", rostri 7", tarsi 7".

Habitat in Brasilia, a Swainson Psaris Cuvieri, (Zoological Illustrations No. 6), a Vieillot Tityris viridis nominatus.

\section{Spéies. 5. PACHYRHYNCHUS NIGER. Tab. XLV。 2.}

Mediocris, supra subtusque niger; capite violaceo-relucente; fasciis 2 alarum albis.

D ESCRIPTro. Corpus praecedente vix minus, totum nigrum, non relucens; caput supra violaceo resplendens, loris genisque nigris; tectrices humerales violaceo splendentes, apice albo-terminatae, alae reliquae nigrae, subtus cinereo-nigrae; cauda subinaequalis, nigra, rectricibus externis apice sordide albo notatis; rostrum plumbeo-nigrum, apice acutum, adurrcum; pedes breviusculi, nigri, haud graciles, digitis haud longis nec connatis. Longitudo corporis $5^{\prime}$, caudae $2^{\prime}$, c. alis $1 . \frac{3}{4}$, rostri $9^{\prime \prime}$, tarsi $8^{\prime \prime}$. 
Mediocris, supra cinereus; subtus rufescens; alis caudaque castaneis ; rostro crasso, nigro.

Descrip т1 0. Corpus praecedente maius, subtus rufescens, in capite et dorso cinereum; genae et regio circa oculos nudiusculae; lora collumque laterale rufa; gu'?a ochracea; pectus abdomenque rufescentia, plumis basi cinereis; crissum femoraque rufa; uropygirm, cauda tectricesque alarum minores et longae castanea, axillares nigricantes; remiges nigricantes, margine externo et interno large rufis, secundariae rufae; alae subtus rufae; cauda aequalis, immaculate castanea; rostrum nigrum, crassum, basi carinato-elevatum, apice aduncum, subtus brunnescens; pedes nigri, argenteo inebulati, subrobusti; digiti laterales basi connati. Longitudo corporis $5 \frac{x^{\prime}}{2}$, caudae $2 \frac{x^{\prime}}{2}$, c. alis 1 , $x^{\prime}$, rostri $10^{\prime \prime}$, tarsi $10 "$.

\section{Spéas 7. PACHYRHYNCHUS RUFESCENS. Ta в. XLVI. 2.}

Minor, supra castaneus, subtus rufesceriti-albus; rostro subtus flavo; alis-immaculatis; remigibus nigricantibus; cauda castanea.

\section{D}

EScriptro. Corpus praecedente fere duplo minus, castaneum, sublus rufó-albescens, plumis basi nigris; caput, dorsum, uropygium, cauda, tectrices, remigesque secundariae suprajacentes castanea; gula, pectus femoraque rufescentia; abdomen, hypochondria crissumque ochraceo-alba; remiges nigricantes, margine externo rufo; interno ferrugineo; alae subtus ferrugineae; cauda aequalis, rectricibus apice triquetris; rostrum plano-convexum, nigricans, subtus ochraceum; pedes haud alti, fusci, albo tecti, digitis basi connatis. Longitudo corporis $4 \frac{3}{4}$, caudae $2^{\prime}$, c. alis $1.1^{\prime}$, rostri $7^{\prime \prime}$, tarsi $8^{\prime \prime}$.

Habitat in Provincia Para; an Muscicapa rufescens Buff, et Lath.? 
Insectis, 'nec' non fructibus victitans, agilis, interdum gregaria, campos arboresque minus altus appetens, vix garrula; cauda alis breviusculis longiore; rostro capite vix breviore, conico, subcylindrico, subcarinato-elevato, parum arouato, baisi triquetro, apice compresso, acuto, dentato, maxilla inferiore a superiore recepta, rectiuscula, compressa, basi altiore; lingua apice fissa; naribus basalibus, impressis, vibrissiș breviusculis; tarsis digito mèdio longô vix maiore, digitis fissis.

\section{SPEC1ES 1. TANAGRA NIGROGULARIS. Ta b. XLVII. M. ı. F. 2.}

Maiuscula; corpore partim nigro, partim purpureo; ophthalmiis, genis, gula, abdomine medio crissoque nigris.

$\mathrm{D}$

ESCR I tr Corpus Fringilla domestica paulo maius, nigrum, purpureo variegatum; caput supra, collum, pectus, hypochondria, dorsum posterius uropygiumque purpurea, relucentia', plumis basi flavo-albicantibus; dorsum anterius, alae, cauda, abdomen medium, crissum, femora, circa oculos, basis maxillarum, apex frontis mentumque nigerrima; plumae capitis quasi truncatae; "alae nigerrimae, subtus antice rubrae; rostrum supra apiceque nigricans, maxilla inferiore basi argentea; pedes nigricantes. Longitudo corporis $6^{\prime}$, caudae $3^{\prime}$, c. alis $1.2^{\prime}$, rostri $9^{\prime \prime}$, tarsi $10^{\prime \prime}$.

Habitat ad flumen Solimoëns in sylvis pagi St. Pauli, foemina pallidius purpurea, alis, cauda abdomineque magis brunne-nigris; differt a T. Jacapa ophthalmiis, genis, mento, dorso superiore, abdomine crissoque haud purpureis sed nigris.

\section{SPEGIES 2. TA NA GRA SAIRA. TÁ. XLVIII. M. 1.}

Maior, supra sulphureo virescens, subtus citrina; rostro crasso, niprro; fronte flavescenti; vertice non crista. to; gula citrina.

$\mathrm{D}_{\mathrm{r}}$ ES Cілтто. Corpus Merula paulo minus, supra sulphurescenti-viride, subtus totum citrinum; caput, praecipue frons et super oculos flavicantia; occiput, nucha, dorsum uropygiumque sulpliurescenti-viridia; lora flavicantia; colli latera, gula, jugulum, pectus, abdomen, femora crissumque citrina, nec non aurantia; tectrices humerales $v i$ rescentes, fusco - nigro rufoque flavo maculatae, reliquae remigesque nigro - fuscae, viridiflavo marginatae, vexillo interno basique alarum luteis; cauda aequalis, alis longior vi-

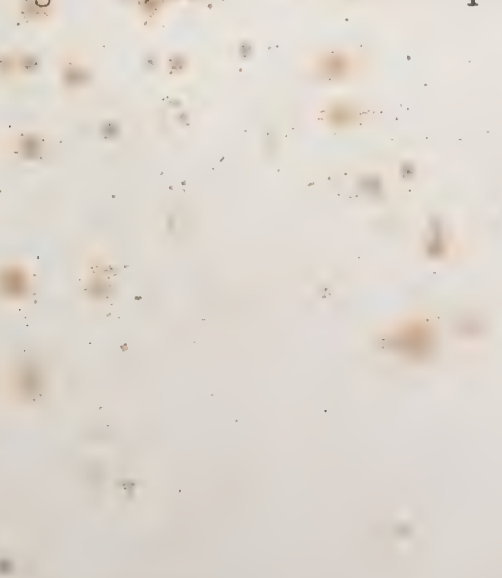
$9^{*}$ 
ridi-fusca, viridi-flavo marginata, subtus sulphurescens; rostrum brunneo-nigrum, crassum, declive, vibrissis nigris brevibus, naribus plumis fere obtectis; tarsi breves, fusci, robusti. Longitudo corporis $7^{\prime}$, caudae $3^{\prime}$, c. alis 1 . $1 \frac{1}{2}$, rostri $9^{\prime \prime}$, tarsi $9^{\prime \prime}$.

Habitat sub nomine, , S a ir a a marella, in campis, sphegibus victitans; foemina fronte vix flavicanti, corporeque subtus minus aurantio; differt a Tanagra olivacea Lath. corpore subtus non albo.

\section{Spegies 3. TANAGRA VIPIDIS. Tab. XlVIII. 2. Mas.}

Maior, supra subtusque viridis, subtus vix sulphurescens; remigibus superioribus caudaque totis viridibus; rostro compresso, vix crasso; pedibus flavo-albis.

$\mathrm{D}$ E S Criptro. Corpus magnitudine praecedentis, supra olivaceo-viride, subtus viridisulphurescens, immaculatum, plumis basi cinereis; caput viride, non cristatum, supra oculos, frontem, genas loraque non flavescens; gula, pectus, abdomen, crissum femoraque unicolora, viridi-sulphurescentia; tectrices alarum, remiges superiores caudaque immaculate olivaceo-virides; remiges primariae apice nigro-fuscae, vexillo externo toto viridi, interno basi fulvo-flavescente; alae subtus antice viridi-flavae; rostrum haud crassum, compressiusculum, acutum, nigricans, maxilla inferiore basi haud altiore, subtus brunnescente; vibrissae nigrae, conspicuae; tarsi robusti, altiores quam in specie praecedente, flavo-albi. Longitudo corporis $6 \frac{3}{4}^{\prime}$, caudae $3^{\prime}$, c. alis $1.2^{\prime}$, tarsi $11^{\prime \prime}$, rostri $9^{\prime \prime}$.

Habitat in Provincia Rio de Janeiro.

\section{Stectes 4. TANAGRA PENICILLATA. Tá. Xlix. I.}

IMaiuscula, supra flavo virescens, subtus aurantia; capite cinerascente; crista verticis dependente olivaceoalba; gula brunneo -alba; rostro brevi, subulato; tarsis gracilioribus.

$D_{\text {s }}$ ECR I Tr O. Corpus praecedente paulo minus, supra flavicanti virescens, subtus saturate et haud rivaciter aurantium; caput brunneo cinerascens, vix olivascens, penicillo verticis dependente, basi albo, apice brunnescenti; gula jugulumque albo brunnescentia; tectrices alarum remigesque secundariae superiores flavo-virides, immaculatae, reliquae remiges nigro-fuscae, margine externo flavo-viridi, interno fulvescenti; 
alae subtus antice aurantiae; cauda aequalis, olivaceo-viridis; rostrum compressiusculum, breviusculum, acutum, brunneo-nigricans, maxilla inferiore pone haud altiore, naribus conspicuis; tarsi breves, brunneo albescentes, digitis gracilioribus. Longitudo corporis $6 \frac{1}{2}^{\prime}$, caudae $3^{\prime}$, c. alis $1.2^{\prime}$, rostri $7^{\prime \prime}$, tarsi $8^{\prime \prime}$.

\section{Species 5. T $\mathrm{T}$ N GRA BRUNNEA. Tab. XliX.2. Mas.}

Mediocris, supra brunnea, subtus brunneo-ferruginea; plumulis verticis longioribus, rubris; rostro brevi, thamnophiloideo sed arcuato; uropygio rufescenti.

$\mathrm{D}$

Escriptio. Corpus Luscinia minus, supra brunneum, subtus ochraceo-ferrugineum, plumis basi nigris; occiput maris nigro cinctum; frons maris nigro submaculata; pennae maris verticis nonnullae rubrae, longiusculae; gula, pectus, abdomen, femora crissumque dilute ferruginea; uropygium rufescens; tectrices alarum brunneae; remiges brunneo-fuscae, margine externo brunneo; alae subtus antice pallide ochraceae; cauda longiuscula, brunnea, margine externo vix rufescenti; rostrum compressum, declive, fusco-brunneum, vibrissis raris, porrectis; pedes breves, nigricantes. Longitudo corporis $6 \frac{I^{\prime}}{4}$, caudae $2 \frac{3}{4}^{\prime}$, c. alis $1.2^{\prime}$, tarsi $9^{\prime \prime}$, rostri $8^{\prime \prime}$.

Habitat in Provincia Rio de Janeiro.

\section{SPEC1ES 6. TANAGRA RUFIVENTER. TAB. L: 1 .}

Mediocris, nigra, subtus et in dorso posteriore fulvo - rufa; vertice flavo; tectricibus scapularibus albis; rostro subfalcato, compresso,

$\mathrm{D}$

E SCrip ti o. Corpus Motacilla alba vix minus, nigrum, subtus totum rufum; caput nigrum, medio aureum; nucha, dorsum anterius, tectrices alarum, femora, nec non remiges et cauda nigra; gula ochracea; jugulum, pectus, abdomen crissumque rufa; hypochondria pallide rufescentia; remiges primariae fusco-nigrae, basi albicantes; alae subtus antice albae; tectrices scapulares albae; dorsum fulvo-ochraceum; uropygium basi rufum, apice nigrum; cauda fusco-nigra; rostrum breviusculum, nigrum; pedes fusco-argentei; Longitudo corporis $6^{\prime}$, caudae $3^{\prime}$, c. alis $1.2 \frac{1}{5}^{\prime}$, rostri $6^{\prime \prime}$, tarsi $7^{\prime \prime}$.

Habitat in sylvis Parae; differt a Tanagra cristata L. crista non purpurea, abdomine non nigro. 


\section{Species 7. үT A A GRA A URIFRONS. тав. L. 2.}

Minor, supra griseo - fuscoque maculata, subtus albescens; fronte maris, praecipue supra oculos, humerisque subtus luteis.

D

E S R г тr o. Corpus Fringilla domestica duplo minus, supra brunnescens, fusco strigilatum, subtus flavescenti âlbum; caput dorsumque olivaceo-grisea, fusco strigilata, striga a genis super oculos, maculaque infra oculos versus mentum aureis; gula, pectus, abdomen, crissum, nec non femora alba, abdomine femoribusque maris vix flavescentibus; tectrices humerales subaureae, reliquae griseae fuscoque strigilatae; remiges brunneo-fuscaè, margine externo vix virescenti, interno basi albicanti; alae sub humero aureae; cauda breviuscula, brunneo-fusca, marginibus vix fulvescentibus; rostrum fringillaceum, conicum, apice compressum, dentatum, in fronte acute carinatum, supra fuscum, subtus flavescens; tarsi subalti, albicantes; oculi grisei. Longitudo corporis 4', caudae $1 \frac{{ }^{\prime}}{2}$, c. alis $1.1^{\prime}$, tarsi $10^{\prime \prime}$, rostri $6^{\prime \prime}$.

Habitat in Provincia Bahia; foemina in fronte, supra oculos et sub alis haud vel vix aurea.

\section{SPECIES 8. TANAGRA SCHRANKII. TaB. LI. Mi. 1. F. 2.}

Exigua, Piprae similis, supra viridi nigroque variegata, subtus aurea; fronte lorisque nigris; vertice maris uropygioque aureo-renitentibus; gula virescenti; hypochondriis virescentibus; rostro brevi.

$\mathrm{D}$

ESC г т ті 0 . Corpus praecedente paulo minus, supra nigrum, plumis viridi fimbriatis, subtus luteum; caput viride nigroque maculatum, fronte, supra nares, lorisque nigris, vertice maris solius luteo; genae gulaque saturate virides; collum laterale, hypochondria, nec non circa anum viridia; femora virescentia; dorsum inferius, uropygium, jugulum, pectus, abdomen medium, nec non crissum lutea; tectrices alarum nigrae, virescenti-coeruleo marginatae; remiges nigrae, primariae margine externo virescenticoeruleo, secundariae viridi; axillae viridi-coerulescentes; alae subtus antice albicantes; cauda breviuscula, aequalis, nigra, rectricibus viridi-coeruleo marginatis, subtus malachitacea; rostrum breve, compressum, Piprae fere simile, dentatum, nigrum; pedes breves, fuscescentes, albo induti, digitis fissis. Longitudo corporis $4^{\prime}$, caudae $1 \frac{{ }^{\prime}}{2}$, c. alis 1. $\frac{3{ }^{\prime}}{}{ }^{\prime}, \operatorname{tarsi} 7^{\prime \prime}$, rostri $6^{\prime \prime}$. 
Foemina vertice non luteo, dorsoque posteriore minus lutescente; differt a Pipra versicolore Temm. pl. 36. F. 2. 3. rostro, dorso uropygioque non cyaneis, fronte juguloque non viridibus, a Tanagra citrinella Temm. Pl. 42. gula haud nigra, dorso non flavo.

\section{Species 9. TANAGRA AURICAPILLA. Taв. LII. M. 1. F. 2.}

Majuscula, olivascens supra, subtus ferrugineo-fulva; vertice maris citrino; alis medio albis, reliquis cau. daque nigris.

$\mathrm{D}$ ES CR 1 P т o. Corpus Fringilla domestica fere maius, supra fusco-olivascens, sub-

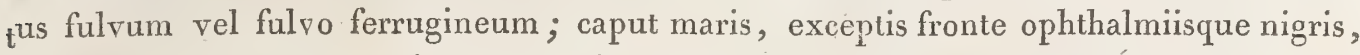
medio totum citrinum, cristatum, reliquum fusco-olivascens, crista luté foeminae vix conspicua; gula, pectus, abdomen crissumque in mare ferrugineo-fulva, in foemina fulvoferruginea; femora olivaceo brunnescentia; tectrices remigesque nigrae, vexillo remigum intermediarum interno medio albo; alae subtus antice albae; cauda aequalis, nigra; rostrum compressum, subarcuatum, nigricans vel brunnescens, lateraliter flavescens; pedes minus alti, fusci, albo tecti. Longitudo corporis $6 \frac{\mathrm{x}}{3}^{\prime}$, caudae $3^{\prime}$, c. alis $1.2^{\prime}$, tarsi $10^{\prime \prime}$, rostri $7^{\prime \prime}$.

Habitat in sylvis Rio de Janeiro, ab Azara N. 101 et a Principe de Neuwied memorata.

\section{Species 10. TANAGRA RUFICOLLIS. Ta}

Mediocris, fringillacea, supra fusco-brunnea, subtus alba; capite maculaqne super aures nigris; striga super oculos et. in medio vertice albicante; collari rufo.

$\mathbb{D}$

E S C I P т r. Corpus magnitudine et fere colore Fringillae domesticae, supra fuscobrunnea, subtus alba; caput nigricans, medio longitudinaliter cinereo-albo striatum; striga a naribus super oculos et versus occiput alba; fascia circa collum rufa; gula, pectus, abdomen crissumque alba; femora brunneo-alba; dorsum anterius brunnescens, nigro strigilatum; tectrices alarum nigro - fuscae, albo vel rufo marginatae; remiges brunneo-fuscae, margine externo albescente; axillae subtus subflavicantes; cauda brunneofusca, subtus cinerascens; rostrum fere subulatum, brunnescens; pedes haud breves, brunnescentes. Longitudo corporis $5 \frac{\mathrm{T}^{\prime}}{2}$, caudae $2 \frac{\mathrm{T}}{4}^{\prime}$, c. alis $1.1 \frac{1}{2}^{\prime}$, rostri $6^{\prime \prime}, \operatorname{tarsi} 10^{\prime \prime}$.

Habitat gregaria in confinibus urbis Rio de Janeiro, Emberizae capitis bonae spei (Buff, T. 5. pl. 386) haud dissimilis. 


\section{Speies 110 TANAGRA CRISTATELLA. Tab. LIII. ז.}

Mediocris, supra nigro-fusca, subtus sordide albescens, plumis basi nigro-cinereis; vertice cristato, purpureo, nigroque cincto; rostro fere fringillaceo.

$\mathrm{D}$

ESCRIPTIO. Corpus Fringilla domestica vix minus, supra dorsum brunnescentinigro-, vel cinereo-fuscum, subtus cinereo albescens, hypochondriis cinerascentibus; caput brunneo cinereoque fuscum, plumis verticis 9" longis, decumbentibus, purpureis, lateralibus nigris; alae brunnescenti nigricantes, immaculatae, subtus sordide albescentes; cauda aequalis, nigra; gula, jugulum, pectus et abdomen intermedia crissumque alba; hypochondria cinerascentia; femora fusca; rostrum subulatum, breviusculum, altum, acutum, supra fuscum, subtus flaro-albescens; pedes haud longi, sanguinei; oculi nigri. Longitudo corporis $5^{\prime}$, caudae $2 \frac{3}{4}^{\prime}$, c. alis 1 . $1 \frac{1}{2}^{\prime}$, tarsi $8^{\prime \prime}$, rostri $6^{\prime \prime}$.

Habitat in sylvis Rio de Janeiro a Tanagra pileata Lath. diversa, a Principe de Neuwied Fringilla pileata, ab incolis, Pimentira Emuing vel Passaro da Crista Vermelha, nominata.

$$
\text { Speq1es 12. +TANAGRA GRAMINEA. Tав. LIII. } 2 .
$$

Mediocris, viridis, subtus sulphurescens; remigibus caudaque nigro-fuscis, viridi marginatis; rostro fere fringillaceo.

$\mathbb{D}$ Ecriptro. Corpus Fringilla domestica paula minus, supra saturate viride, subtus totum flavescens; caput totum coerulescenti-viride, vix nigro striatum; gula viridi flavescens; femora fuscescentia; tectrices alarum vix nigro-fuscae, sed virides; remiges nigro-fuscae, margine externo virescenti; cauda haud longa, nigro-fusca, rectricibus, praecipue intermediis large viridi marginatis, subtus chalybeis; rostrum nigricans, conicum, breve, altum, apice tenue, marginatum; pedes flavescentes. Longitudo corporis $4 \frac{3}{4}^{\prime}$, caudae $2^{\prime}$, c. alis 1. $1^{\prime}$, tarsi ' 9", rostri 6". 


$$
\text { Wind }
$$

Srecies iz. TANAGRA CAPISTRATA. тав. Liv. i.

Maiuscula, supra cinerascens, subtus tota ferruginea; capite brunnescente; fascia basi rostri nigra; abdominê medio axillisque albis.

\section{D}

Es criт ті Co. Corpus Merula minus, supra cinereo plumbescens, subtus ferrugineorufum, plumis basi nigro-cinereis; caput supra olivaceo-brunnescens, loris ferrugineis; regione frontis genaeque versus mentum nigris; gula, pectus, abdomen anterius crissumqute rufo ferruginea; abdomen medium axillaeque alba; hypochondria plumbescentia; femora albo-cinerea; tectrices alarum plumbescentes; remiges nigro-fuscae, margine externo plumbescente; cauda longiuscula, aequalis, cinerascenti-nigra, apice albescens; rostrum crassum, breviusculum, plumbescens; pedes nigri. Longitudo corporis $6 \frac{3}{4}{ }^{\prime}$, caudae $3^{\prime}$, c. alis $1.2^{\prime}$, rostri $6^{\prime \prime}$, tarsi $9^{\prime \prime}$.

Habitat in sylvis Rio de Jan e iro, a Principe de Neuwied designata.

Species 14.+TANAGRA AXILLARIS. TAв. LIV. 2. Mas.

Maiuscula, sordide cinerascens, subtus alha; loris axillisque nigris; tectricibus alarum albo fasciatis; remigibus brunnescentibus; cauda nigricante, subtus obscure nigro fasciata; striga alla super oculos nulla.

D Eșcirptio. Corpus magnitudine praecedentis, supra cinerascens, brunneo vix nebulatum, subtus album; caput dorsumque eiusdern coloris; aures nigro màculatae; colli latera, gula, abdomen, hypochondria, femora crissumque alba; pectus sordide albescens, cinereo nebulatum; tectrices alarum anteriores nigrae, medio large albo-fasciatae, posteriores brunnescentes; remiges secundariae superiores brunnescentes, reliquae nigro-fuscae, margine externo brunnescente; alae ad marginem axillarem et subtus antice albae; cauda aequalis, basi rectriceque intermedia brunneo-fusca, versus apicem nigricans, subtus obscure nigro-fasciolata, plumbeo terminata; rostrum breviusculum, subcrassum, supra brunnescens, subtus basi plumbescens, apice albescens; pedes brunnei, griseo obducti; oculi nigri. Longitudo corporis $6 \frac{{ }^{\prime}}{4}$, caudae $2 \frac{3{ }^{\prime}}{4}$, c. alis $1.2^{\prime}$, rostri $7^{\prime \prime}$, tarsi $9^{\prime \prime}$. 
Majuscula, tota coerulescens; humeris albis; alis caudaque azureis.

$\mathrm{D}$

ES CR т т1 O. Corpus Fringilla domestica vix maius, supra, praecipue subtus dilute coerulescens vel colore marino relucens; caput totum, gula, pectus, abdomen, femora, crissum uropygiumque colore marino vel dilute coerulescentia; tectrices humeri albae, vix coeruleo cinctae, reliquae saturate azureae, apice albo fasciatae; remiges nigrae, vexillo externo saturate azureo; alae subtus antice albae; cauda aequalis, supra saturate azurea, subtus coerulescenti chalybea; rostrum triquetrum, breviusculum, basi cocruleo albescens, reliquum nigrum; oculi grisei; pedes breves, nigricantes, albo tecti. Longitudo corporis $6^{\prime}$, caudae $2 \frac{I^{\prime}}{2}$; c. alis 1 . $1 \frac{T^{\prime}}{4}$, rostri $6^{\prime \prime}$, tarsi $9^{\prime \prime}$.

Habitat Parae et ad fl. Solimoëns prope Pagum Fonteboa; foemina minor capite abdomineque magis canescentibus, tectricibus humeri minus albis coeruleșcentique albicantibus, rostro paulo longiore supra fuscescente, subtus flavicante.

Spezies 16. TANAGRA ARCHIEPISCOPUS. Taz. LV، I. Mas.

Maiuscula, azurea; alis caudaque virescentibus; tectricibus humeralibus azureis, medio flavo fasciatis; dorso nigricanti-azureo.

$\mathrm{D}$ ESCR I T I 0。 Corpus praecedente paulo maius, supra dorsum nigricanti coerulescens nec non virescens, subtus et in capite colloque azureum; genae versus nares nigricantes; femora crissuinque sordide albescentia vel grisea; humeri azurei, pone large flavo fasciati; tectrices alarum reliquae remigesque nigricantes, margine externo virescenti; alae subtus albae; cauda supra subtusque nigricans, margine rectricum externo praecipue intermediarum virescenti; rostrum nigrum, tenue, haud breve, basi carinatum; oculi rubro-flavi; pedes nigricantes. Longitudo corporis $7^{\prime}$, caudae $3^{\prime}$, c. alis 1 . $1 \frac{\mathrm{I}^{\prime}}{2}$, rostri $8^{\prime}$, tarsi $9^{\prime \prime}$.

Habitat in locis sylvaticis Rio de Jan eix 0. 
SPECies $17 .+$ TANAGRA RUBRICOLLIS. Taв. LVI. Foem. 1.

MIaior, violaceo-nigra, gula juguloque purpureis.

D

Ecriptro. Corpus magnitudine Merulae, supra subtusque immaculate violaceo-nigrum, plumis basi albicantibus; gula maris, jugulum pectusque anterius purpurea; alae supra subtusque violaceo-nigrae, apice vix fuscescentes; cauda alis longior, aequalis, supra violaceo - nigra, subtus nigra; rostrum capite vix brevius, nigrum, arcuatum, subcylindricum, apice acutum; pedes haud alti, robusti, nigri. Longitudo corporis $7 \frac{1^{\prime}}{2}$, caudae $3^{\prime}$, c. alis l. $1 \frac{I^{\prime}}{4}$, rostri $9^{\prime \prime}$, tarsi $9^{\prime \prime}$.

Habitat in sylvis compestribus Bahiam inter et Rio de Janeiro; Foemina gula juguloque modo purpureo nigroque mixtis; mas a Buffonio (T. V, pl. 381 ) sub nomine Muscicapae Rubricollis depictus.

\section{Spscies 18. TANAGRA ATRICOLLIS。 Tab. LVI. Foem.2.}

Maior, brunnescens supra, subtus ferruginea; cauda ferruginea, subtus obscure nigro fasciata; genis, gula juguloque nigris; axillis albicantibus; rostro crasso, ochraceo.

D

ESGRIPTio. Corpus magnitudine fere Merulae, supra brunnescens, subtus dilute ferrugineum, plumis basi cinereis; caput supra brunnescens, ante, infra, nec non pone oculos nigrum; gula jugulumque nigerrima; colli latera brunneo-cinerascentia; pectus, abdomen femoraque dilute, crissum ac hydochondria magis ferruginea; tectrices alarum humerales fusco-brunneae, apice vix cinerascentes, axillares marginales albescentes, scapulares rufo-brunneae; remiges nigro-fuscae, margine externo brunnescente; alae subtus antice subalbicantes; cauda longiuscula, nigricans, apice brunnescens, subtus nigro - fasciata, rectricibus 2 intermediis supra nigro-brunnescentibus; rostrum altum, longiusculum, subcompressum, supra angustius, fuscescens, reliquum ochraceum; oculi grisei; pedes flavicantes, alti, robusti. Longitudo corporis $7 \frac{3}{4}$, caudae $3 \frac{3}{4}$ ', c. alis 1. $2 \frac{1^{\prime}}{3}$, rostri $9^{\prime \prime}$, tarsi $\mathbf{1}^{\prime}$.

Habitat in campis Provinciae $\mathrm{M}$ in as Gera ës. 


\section{Speges ig. TANAGRA SUPERCILIARIS. Tá. LVIr. 1. Mas.}

Maxima, fusco-cinerea, subtus albescens; striga super oculos aliaque gulari albis, alia a mento utrinque ad collum nigra; pectore albo-cinereo; abdomine crissoque rufis.

D E SCriptio. Corpus magnitudine Merulae, supra cinereo-fuscum, olivaceo vix nebulatum; caput fusco-cinereum, striga a naribus super oculos alba; genae loraque fuscocinerea; striga a basi maxillae inferioris juxla gulam nigra; gula jugulumque in medio alba; pectus albo - cinereo obfuscatum; hypochondria, abdomen crissumque rufescentia; femora canescenti-fulva; tectrices alarum brunnescenti nigricantes; remiges secundariae superjacentes brunneo-fuscae, margine externo haud raro virescenti, reliquae fusconigrae, margine externo canescente; axillae alaeque subtus antice albescentes; cauda subgradata, fusco nigricans; rostrum crassum, altum, longiusculum, dentatum, brunneonigricans; oculi grisei; pedes fusci, haud breves. Longitudo corporis $8 \frac{11}{3}$, caudae $4^{\prime}$, c, alis $1.2 \frac{1}{2}^{\prime}$, rostri $8^{\prime \prime}$, tarsi $3^{\prime}$.

Habilat in campis fl. St. Francisci prope pagum Joaze iro; differt a Tanagra magna Lath. corpore supra non viridi juguloque non ferrugineo.

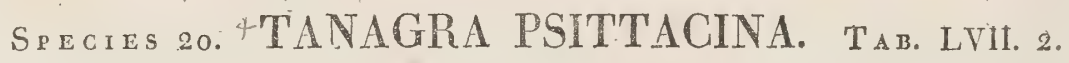

Maior, tota nigra, plumbescenti induta; rostro quam maxime crasso, alto, convexo, uncinato ferrugineogue; alis supra fuscescentibus, subtus albis.

Descriptro. Corpus Merula haud minus, nigrum, hinc inde, praecipue supra plumbeo tinctum; caput nigro plumbescens, ante oculos fere nudum; tectrices humerales plumbescentes; reiniges brunneo nigricantes, subtus basi albescentes; axillae albicantes; cauda fusco-nigra; gula, pectus, abdomen femoraque fuscescenti nigra, vix plumbescentia ; rostrum fere psittacinum, conicum, quam maxime crassum et altum, supra rotundato-convexum, nigro-fuscescens, lateraliter emarginatum, apice dentatum, subhamalum, naribus vibrissis nonnullis nigris tectis; pedes vix breves, brunneo-fusci, robusti. Longitudo corporis $8^{\prime}$, caudae $4^{\prime}$, c. alis $1.2 \frac{1}{2}^{\prime}$, rostri $11^{\prime \prime}$, tarsi $1^{\prime}$ 。

Habitat gregaria in sylvis Rio de Jan eiro proximis. 


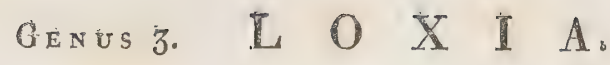

Familiarìis, granivora, agilis; rostro brevi, conico, apice acuto, frequenter emarginató, basí non angustato et compresso sed crasso, latiusculto, supra plano-convexo, subarcuato, maxilla inferiore fere psittacea, magna, raro compressa, angulata, margine superiore introrsum subconnivente, naribus subsuperis, basalibus, frequenter divaricatis, fere tèctis, vibrissis vix utlis; tarsis digito medio vix altioribus.

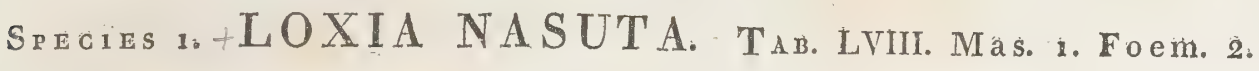

Minor, immaculata, mas supra et in gula niger, subtus castaneus, foemina supra brunnea, subtus rufescens; rostro tumido; alis subtus albis。

D

ESCR IP Tि O. Corpus Fringilla cardueli paulo minus, in mare supra totum nigrum absque splendore violaceo, subtus saturate castaneum, exceptis gula, jugulo pectoreque anteriore nigris, in foemina supia brinneum, alis caudaque nigro-fuscis, subtus rufescens; remiges basi alaeque subtus antice albae; rostrum crassum, breve, conicum, vix emarginatum, basi tumidum, in mare fusco flavescens, in foemina nigricans et paulo minus tumidum; pedes fusco-sanguinei. Longitudo corporis 4 $4 \mathbf{x}^{\prime}$, caudae $2^{\prime}$, c. alis 1. $1 \frac{1}{2}$, rostri $5^{\prime \prime}$, tarsi $8^{\prime \prime}$.

Habitat in confinibus $\mathrm{P}$ ar a

\section{Species 2. LOXIA LEUCOPTERYGIAa. Tab: LVIII.}

Exigua, supra violaceo - nigra, subtus alba; remigibus medio albo-fasciatis; pectore nigro-vittato; gula alba; uropygio albo-cinereo; tectricibus humeri albo nigroque variegatis; rostro stramineo.

D ESCR1Pті C. Corpus praecedente fere maius, supra nigrum, violaceo subrelucens, subtus, excepto pectore, album; caput violaceo-nigrum; pectus anterius nigrum, albo maculatum; tectrices humeri caudaeque supra nigro-violaceae, cinereo-albo terminatae; remiges nigro-fuscae, medio basique albae; cauda fusco-nigra, à ice vix fulvescenti fimbriata; dorsum inferius uropygiumque albo-cinerea; gula, pectus inferius, abdomen, hypochondria, femora crissumque alba, plumis basi cinereis; femora infra fusca; rostrum stramineum, subarcuatum, superius inferiore paulo crassius; pedes sanguinolenti; oculi grisei; Longitudo corporis $4 \frac{t^{\prime}}{3}$, caudae $2^{\prime}$, c. alis $1.1^{\frac{1}{2}}{ }^{\prime}$, rostri $5^{\prime \prime}$, tarsi $8^{\prime \prime}$,

Habitat in vicinitate $\stackrel{\mathrm{P}}{\mathrm{P}} \mathrm{ra}$ e ; differt a Loxia nitente (Buff. pl. 224) colore supra minus, subtus non violaceo, rostro nec fringillaceo nec compressiusculo. 
+ SPECIES 3. LOXIA ALBOGULARIS. TA b. LX. Mas. 1. Foem. 2.

Minor; gula, alis mediis abdomineque medio albis; rostro rubro, exiguo; mas supra fusco-nigricans, subtus, excepto pectore, nigricanti - albus; foemina supra clare brunnea, subtus cinereo - alba-

$\mathrm{D}$ EScriptro. Corpus praecedente minus. Mras: caput posticum, dorsum uropygiumque fusco-cinerea; frons, genae, lora pectusque superius nigricantia; gula, pectus inferius, abdomen medium crissumque alba; hypochondria cinerea; femora nigro alboque variegata; alae fusco-nigrae, medio basique albae; cauda fusco-nigra; tarsi sanguinolenti.

Foemina: caput, dorsum, uropygium brunnea; gula, abdomen medium crissumque alba; pectus sordide vel cinereo albescens; remiges brunneo-fuscae, margine externo fulvescente; cauda brunneo - fusca.

\section{Species $4 .+$ LOXIA IGNOBILIS. Tab. LX. 3.}

Minor, subtus fulva, alis caudaque fusco brunnescentibus; mas supra olivaceo - virescens, foemina brunnescens.

$\mathrm{D}$

ESCR 1PTIO. Corpus praecedente paulo maius, MIas: caput, dorsum, uropygium tectricesque nonnullae alarum olivaceo virescentia; lora viridi-fulvescentia; gula, pectus femoraque rufescenti - fulva; abdomen crissumque albo fulvescentia; remiges fusco brunnescentes, margine externo olivaceo - virescenti, apice fuscescens; alae subtus antice albo-fulvescentes; cauda fusco-brunnea, olivaceo - viridi induta; rostrum breve, planoconvexum, brunneo-albescens; pedes fusci. Longitudo corporis $4 \frac{I^{\prime}}{3}$, caudae $1 \frac{3^{\prime}}{4}$, c. alis 1. $1^{\prime}$, rostri $4^{\prime \prime}$, tarsi $6^{\prime \prime}$.

Habitat in Provincia P ara; foemina supra magis brunnescens et vix olivascens.

$$
\text { Species } 5 .+ \text { LOXIA PLEBEJA. Tab. LIX. } 3 \text {. }
$$

Exigua, olivaceo-brunnea; gula pectoreque nigris; abdomine flavescenti-albo; alis mediis albo non-fasciatis; rostro albescente.

Descriptio. Corpus praecedente vix minus, supra fere fusco-brunneum; frons nigricans; gula, jugulum pectusque nigra; uropygium femoraque olivaceo-brunnea; 
pectus inferius, abdomen crissumque flavo-alba; alae caudaque fusco-brunneae; alae subtus albae; humeri subtus virescenti-brunneo alboque variegati; rostrum breve, albicans; pedes sanguinolenti.

\section{Speries 6。 LOXIA BREVIROSTRIS.Tab, LIX, Mas, „Foem،2.}

Minor, supra subtusque rufa; capite maris supra, alis caudaque nigricantibus.

D ESCRIPT10. Corpus praecedente minus, supra subtusque in foemina brunneorufescens, in mare rufum; M a s: caput supra nigrum, postice, supra aures et infra oculos rufum; dorsum, uropygium, gula, pectus, abdomen, femora crissumque rufa, plumis basi nigro-cinereis; tectrices alarum nigricantes, fulvo subterminatae; remiges nigrae, medio basique albae; cauda nigra, apice vix pallescens; rostrum minutum, breve, cinereo-fuscum; pedes breves, brunnescentes. Longitudo corporis $3 \frac{1}{2}^{\prime}$, caudae $1 \frac{I^{\prime}}{2}$, c. alis 1. 1", rostri $3^{\prime \prime}$, tarsi 6".

Habitat in confinibus Para e; foemina tota brunneo-rufescens.

\section{Genus 4. F R I N G I L L A.}

Gregaria, granivora, garrula, in deviis et ad domicilia habitans; rostro conico, subulato, compressiusculo, non arcuato, nec basi crasso, nec supra plano-convexo sed angustato, naribus approximatis; mandibula inferiore basi minus alta, margine superiore introrsum connivente; vibrissis nullis; tarso digito medio fere altiore.

Spenes 1. FRINGIILA BRASILIENSIS. Tав. LXI. Mas, 1, Foem. 2.

Mediocris, supra flavo-virescens, fusco strigilata, subtus citrina, capite maris aurantio.

D E S с в г т 1 . Corpus foeminae Fringillae Canariae simillima, supra flavo-virescens, fusco strigilatum, subtus totum citrinum; remiges nigricantes, viridi-flavo limbatae; alae subtus antice luteae; cauda fusco-nigricans, emarginata, margine externo et interno rectricum flavescenti, subtus lutea; uropygium luteum; rostrum haud longum, conicum, albescens; pedes sordide albescentes. Longitudo corporis $4 \frac{1}{2}^{\prime}$, caudae $2^{\prime}$, c. alis $1 . \frac{1}{2}^{\prime}$, tarsi $8^{\prime \prime}$, rostri $5^{\prime \prime}$.

Habitat in campis Minas Geraës, Canario nominata; foemina a Buffonio sub nomine Emberizae brasil. depicta (T. 5. Pl. 321. F. 1.) magis virescens capiteque vix aurantio. 


\section{Sretes 2. FRINGILLA CAMPESTRIS. Taв. LXI. 3. Mas.}

Minor, supra sulphureo virescens, subtus sulphureo flavicans; alis medio caudaque basi flavo - fasciatis; remigibus caudaque nigris.

D

ESC R P т I o. Corpus praecedente multo minus, snpra flavo-viride, subtus sulphureum; caput viride, vix fuscescens; tectrices alarum nigrae, viridi-flavo terminatae; remiges nigrae, medio basique citrinae, lateraliter viridi-, apice albicanti marginatae; alae subtus flavae; cauda nigra, apice emarginata, basi flava, lateraliter vix viridi mar. ginata; uropygium virescens, supra flavum; gula pectusque viridi-sulphurea; abdomen, femora crissumque flava; rostrum conicum, acutum, compressum, fuscescens; pedes fusco-sanguinolenti; oculi nigri. Longitudo corporis $4^{\prime}$, caudae 1 $\frac{x}{2}$, c. alis 1 . $7^{\prime \prime}$, ros$\operatorname{tri} 5^{\prime \prime}$, tarsi 6".

Habitat in campis districti adamantini, Sa hy B icudo nominata.

\section{Familia: G A L L I N A E.}

Familiares, brachypterae, longicaudatae, ad volatum altum ineptae, grani-vel baccivorae, in locis sylvaticis nec non paludosis super arbores vel in terra nidificantes, facile mansuefaciendae; corpore ponderoso; collo exserto; rostro conico, versus apicem modo unguiculato, subtumido, incurvo, non dentalo; mandibula inferiore superiore fere breviore, aeque alto, naribus fere intermediis, apricis, impressis, ovatis, membrana tectis; digitis anticis basi semipalmatis tarso robusto, antrorsum scutellato-fasciato, usque infra genua plumoso brevioribus, postico terrae incumbente.

\section{Genus I. $\mathrm{G} R \mathrm{R} \cdot \mathrm{X}$.}

Sylvatica; in locis humidis super arbusta nidum e ramis construens, voce vix fritinniens; cri= sta capitis plerumque alta, erectili; rostro valido, capite vix breviore, gibboso-convexo, altiore quam latiore, apice aeque fere alto, incurvo; naribus transversis, membrana larga tectis; trachea longitudinaliter revoluta; digito postico haud brevi.

\section{Stecres 1。 CRAX FASCIOLATA. Tab. LXila。}

Mediocris, nigra, fulvo fasciolata; collo nigro, vix fasciato; crista capitis albo-fasciata; pectore, abdomine, crisso femoribusque ferrugineis; rostro flavo - fusco, haud albo; pedibus vix rubicundis.

Descrepro. Corpus magnitudine Gallinae domesticae, nigrum absque violaceo; crista capitis nigra, $1 \frac{\pi^{\prime}}{2}$ alta, fulvo-fasciata; collum nigrum, infimum fulvo-fasciatum; 
pectus, nec non femora ferruginea, nigro maculata vel fasciata; reliquum corpus nigrum, fulvo fasciolatum; rostrum subarcuatum, basi flavescens, apice plumbescens, naribus patulis, intermediis.

Habitat in sylvis Parae.

$$
\text { Species 2. + GRAX URUMUTUM. Taв. LXII. }
$$

Màgna, supra subtusque castaneo - rufa, circa oculos coerulescenti-flava; rostro rubro; dorso tectricibusque alarum rufo nigroque undulatis; crista capitis caudaque nigris; cauda nigra, apice albo-fasciata.

D ESCR I t т o. Corpus gallina domestica vix maius; crista capitis $1 \frac{3}{4}$ ' longa, recumbens, apice vix rufescens; frons rufescens; regio circa oculos et versus nares nuda, coerulescenti-viridi flava, supra oculos auresque rufescens; collum, gula, pectus, abdomen, crissum femoraque castaneo-rufa; dorsum, uropygium, tectrices alarum, remiges, praecipue secundariae vexillo externo, rectricesque intermediae fusciora, tenerrime nigro rufoque transversim undulata; alae subtus antice rufescentes; remiges brunneo nigricantes, relucentes; cauda longiuscula, subaequalis, subviolaceo-nigra, apice albo fasciata; rostrum haud longum, fornicatum, rubrum, basi vix nigricans; oculi castaneo-brunnei; pedes robusti, plumbescenti virescentes, lateraliter castaneo vix induti. Longitudo corporis $2^{\circ} 1^{\prime}$, caudae $8 \frac{1}{4}^{\prime}$, c. alis $1.5 \frac{\mathrm{I}^{\prime}}{4}$, rostri $1 \frac{1}{2}^{\prime}$, tarsi $3 \frac{1}{2}^{\prime}$.

Habitat in sylvis fl. Nigri, Urumutum nominata, interdum in cortes recepta.

\section{Speine 3. CRAX TOMIENTOSA. Taв. LXIII.}

Maiuscula, nigra, violaceo subrenitens, non cristata; rostro alto, aquilino; plumis capitis non cristati collique superioris tomentosis, quasi truncatis; crisso, femoribus supra, caudaeque nigrae apice castaneis.

D ES Cв 1ртіо. Corpus praecedente maius, Cr. Rubrirostri vero minus, supra subtusque, exceptis abdomine, crisso caudaeque apice rufo-castaneis, violaceo-nigro chalybeum; caput non cristatum, nigerrimum, circa oculos et pone nares tomentoso-plumosum, plumis reliquis brevibus et fere truncatis; collum elongatum, vix crassuin; tectrices nuchae collique inferioris nigro - violaceo splendentes; alae fuscescentes; dorsum pectusque nigra, fere absque splendore violaceo; alae subtus atrae; abdomen infimum crissumque maris et foeminae rufo-castanea; femora nigra, raro rufo-maculata ; cauda elongata, violaceonigra, apice rufo-castaneo fasciata; rostrum breve, altum, compressum, aquilinum, 
rubrum, apice flavescens, supra fuscescens, naribus. basalibus; tarsi pallide rubicundi.

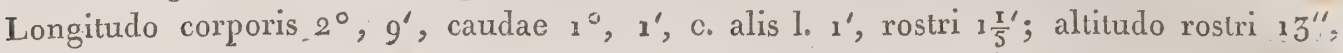
tarsi $4 \frac{1}{2}^{\prime}$.

Habitat Cr. Pauxis rostro affinis in sylvis paludosis Fl. Nigri prope pagum Barcellonam.

\section{Species 4. CRAX BLUMENBACHII. Tab. LXIV.}

Major, violaceo-nigra ; alis rufo nigroque fasciolatis; abdomine, crisso nec non femoribus rufo-castaneis; crista capitis crispa, albo-fasciata; cauda gradata, tota violaceo-nigra.

Descripтіо. Corpus Cr. Rubrirostri paulo minus, violaceo-nigrum, vix chalybeum; crista capitis nigra, $\mathbf{1} \frac{\mathbf{x}^{\prime}}{2}$ alta, apice antrorsum vix reflexa ac haud crispa, medio albo 5 fasciata, pennis non angustis; caput circa oculos tomentosum, pone oculos vix nudum; gula nigra, plumis basi albo-guttatis; pectus nigrum; abdomen anterius hypochondriaque nigra, rufo maculata; abdomen posterius crissumque rufo-castanea; femora rufo-castanea, basi plumisque apice nigro-fimbriatis; tectrices humerales nigrae, axillares, posteriores remigesque omnes supra et subtus rufo nigroque transversim undulatae; cauda elongata, gradata, supra virescenti-violaceo nigra; rostrum minus altum, basi brunneo-fuscum, apice brunneo-albescens, naribus intermediis, largis; pedes fere rubicundi. Longitudo corporis $2^{\circ}, 9 \frac{1}{2}^{\prime}$, caudae $x^{\circ}, c_{+}$alis $1 . x^{\prime}$, rostri $1 \frac{1}{2}^{\prime}$; altitudo rostri $1^{\prime}$, tarsi $4^{\prime}$.

Habitat in sylvis Provinciae Rio de Janeiro. [irror, Gutr amer.?]

Species 5. CRAX GLOBULOSA. Tab. LXV. Mas. LXVI. Foem.

IMaior, tota violaceo-nigra, cristata, flavirostris; mandibula inferiore aequali basique non altiore; mas globula supra nares unico, carunculis 2 globulosis infra maxillam; crisso et regione circa anum albis; foemina nec globicera nec caruncalata, crisso rufo.

Descriptro. Corpus praecedente vix maius, supra subtusque violaceo-nigro chalybeum; crista capitis $1 \frac{1}{2}$ alta, nigra, apice antrorsum reflexa ac crispa, in foemina raro medio albo-fasciata, pennis angustatis; regio circa et ante oculos nudiuscula, supra aures tomentosa; collum, dorsum, pectus, hypochondria, femora, alae caudaque nigroviolaceo nec non virescenti chalybea; abdomen superius fusco-nigrum, lateraliter interdum rufescenti transversim lineolatum; abdomen maris circa anum, crissumque albissima, 
foeminae rufa; femora nigra, foeminae parum rufo maculata; rostrum subcompressum, longiusculum, basi flavicans, apice nigricans, mandibula inferiore aeque alta; globulus flavus, supra nares intermedius, $i_{4}^{3}$ altus, carunculaeque inframaxillares globulo nasali minores, aeque flarae, globulo nasali carunculisque in foemina deficientibus; pedes rubicundi, digitis fuscescentibus. Longitudo corporis $2^{\circ} 6^{\prime}$, caudae $1^{\prime}$, c. alis $1.10^{\prime}$, rostri $2 \frac{1}{3}^{\prime}$; altitudo rostri absque globulo nasali $1^{\prime}$, mandibulae inferioris $3^{\prime \prime}$, tarsi 4'.

Habitat in Sylvis fl. Solimoëns; an Cr. Globicera L a th. et Buff. (T.2. pl.86.), quam carunculis mandibularibus destitutam describunt? an Cr. carunculata Tem. (pag. 44.) globulo nasali non instructa?

\section{Species 6. CRAX RUBRIROSTRIS. Tab. LXVII.}

Maior, violaceo-nigra, chalybea, cristata; rostro basi purpureo; abdomine, circa anum crissoque albis; cauda tota violaceo - nigra.

$\mathbb{D}_{\mathrm{E}}$ Scriptro. Corpus praecedente maius, totum violaceo-nigro chalybeum, aeneonitens; crista capitis tota nigra, $1 \frac{I^{\prime}}{2}$ alta, plumis tenuibus, apice antrorsum flexis, crispis; regio circa oculos nudiuscula, reliqua uti et colli superioris tomentosa; alae supra subtusque violaceo-nigrae, rufo nequaquam fasciolatae; cauda subgradata, violace - nigro virescentique renitens; abdomen, regio circa anum crissumque albissima; femora nigra; rostrum longum, nec globulo nec carunculis ornatum, basi purpureum, apice nigrum; pedes robusti, nigri, rubro leviter induti. Longitudo corporis $2^{\circ} 6^{\prime}$, caudae $1^{\circ} 1^{\prime}$, c. alis $1.9^{\prime}$, rostri $2^{\prime}$, tarsi $3 \frac{1}{3}^{\prime}$.

Habitat in sylvis $\mathrm{fl}$. Amazonum et inter Rio de Janeiro ac Bahiam, bahiensis maxilla inferiore basi altiore ac subtus sinuata; differt a Cr. Alectore Lath. corpore non rubro, ab Alectore $\mathrm{Tem}$., quem nigrum describit, rostro non flavo.

\section{Stecies 7.t C R A X T U B ER OS A. Tab. LuviIa.}

Maxima, cristata, violaceo-nigro resplendens; rostro rubro, crasso, basi tuberoso; crisso rufo; cauda nigra, apice albo-fasciata; naribus basalibus, fere tectis; plumis cristae capitis latis, nec crispis nec revolutis.

$\mathrm{D}$ E SCR I т́ т o. Corpus praecedentibus maius, violaceo-nigrum, praecipue superne plumis squamosis, violaceo splendentibus; caput crassum, cristatum, crista verticis $1 \frac{1}{2}$ longa, depressa, pennis rarioribus, latis, nec crispis nec reflexis; latera capitis et circa oculos 
nec non collum breviusculum et crassum tomentosa; abdomen inferius crissumque rufocastanea; femora hypochondriaque nigra; alae caudaque violaceo-nigrae, cauda apice albo fasciata; rostrum haud longum, crassum, altum, ante frontem parum sulcatum, pone convexo-planiusculum et lateraliter tuberosum, naribus pressis, basalibus; pedes robusti, altiores, rubicundi. Longitudo corporis $2^{\circ} 7^{\prime}$, caudae $1^{\circ} 1^{\prime}$, c. alis $1.10^{\prime}$, rostri $2^{\prime}$; altitudo rostri $1^{1^{\prime}}{ }^{\prime}$, tarsi $4^{\prime}$.

Habitat in sylvis fl. Soli mo ëns; differt a Pauxi Mitu Temm. (pl. 153.) rostro versus frontem non acuminato et cultrato sed sulcato et tuberoso.

\section{GExus 2. P E $\mathrm{N}$ E L O P E.}

Familiaris, in sylvis paludosis supra arbores habitans, matutine et vespere ejulans; plumis capitis non cristatissed erectilibus; rostro nec crasso nec alto, rectiusculo, apice deflexo, basi carinato, circa oculos, aures, nares, nec non versus collum anticum nudo, naribus impressis patulis, membrana larga tectis; digito postico haud brevi.

\section{Stétes I.+PENELOPE JAC QÚUAC, U. Tab. LXVIII.}

Maxima, virescenti-aenea; plumis basi.rufis; gula nec non jugulo nudis, rubicundis; regione circa oculos, aures naresque nuda, violacea; capite lorisque virescenti-fuscis, albo non maculatis ac strigilatis; pectore, abdomine, crisso femoribusque rufis; tectricibus juguli albo-submarginatis, alarum fere immaculate virescenti-aeneis; cauda supra rufo virescentique aenea, subtus violaceo-nigra.

D E S C I P T1 O. Corpus magnitudine Phasiani Nycthemeri, fuscum, virescenti-aeneum; caput supra et circa oculos, nares auresque nudum, violaceo nitens, plumis supra aeneofuscis, albo non strigilatis, erectilibus; mystax a mandibulae inferioris basi infra aures brunneo-fuscus, albo non maculatus, tenuis; gula nuda, vix pilis raris obsita, rubicunda, medio longitudinaliter valvuloso-compressa, valvula dilatabili; collum posterius fusco-virescenti aeneum, albo non maculatum; jugulum inferius pectusque superius fusca, virescenti-aenea, plumulis albo fimbriatis; tectrices alarum aeque virescenti-aeneae, fulvo haud vel vix fimbriatae; remiges fuscae, viridi-aeneo renitentes, subtus brunnescenti-nigrae; cauda subgradata, longa, viridi-aeneo-, nec non rufescenti et violaceo-renitens; subtus violaceo-nigra; pectus, abdomen, crissum femoraque rufa; femora infra fusciora; uropygium fusco rufescens rostrum brunneo-fuscum; non altum, rec- 
tum, apice declive, supra nares tenue ac carinatum; pedes rubicundi; oculi rubri. Longitudo corporis $2^{\circ} 6^{\prime}$, caudae $1^{\circ}, 1^{\prime}$, c. alis $1.10^{\prime}$, rostri $1 \frac{1}{2}^{\prime}$; altitudo rostri $7^{\prime \prime}$, tarsi $3^{\prime}$.

Habitat in sylvis fl. Solim ö ns, foemina raro tectricibus alarum albo-fimbriatis; Penelope cristata scriptorum nonnullorum.

\section{Speries 2.+PENELOPE JACU-CACA. Tas. LXIX, Foem。}

Praecedente vix minor, nigricantifusca, virescenti-aeneo relucens, plumis basi fuscis; collo, praecipue cau= da violaceo-aeneis; striga super oculos et ad latera colli albicante; plumis verticis, tectricum alarum, nec non juguli et pectoris lorisque albo fimbriatis; pectore, abdomine, crisso femoribusque non rufis sed nigro - fuscis; gula media nigro - pilosa.

$\mathrm{D}$ ESCRIPTIO. Corpus magnitudine fere praecedentis, colore vero magis nigrofusco; caput circa oculos et nares nudum, rubro-violaceum; plumae capitis triquetrae, albo-fimbriatae, erectiles; striga super oculos nigra, aliaque super oculos et latera colli alba, parum fusco maculata; frons apice nigra; lora haud tenuia, usque post aures nigricantia, albo immixto; gula vel jugulum superius nudum, lateraliter pilis nigris raris, medio confertis obsitum; collum posterius fusco-aeneum, violaceo renitens, albo non maculatum; jugulum, pectus, nec non abdomen nigro-fusca, aeneo violaceoque renitentia; femora crissumque atrofusca; tectrices alarum fusco-aeneae, violaceo renitentes, margine laterali albo-fimbriato; remiges fusco-aeneo-virescentes, scapis rufescentibus; cauda longa, subgradata, violaceo renitens, rectricibus intermediis virescenti aeneis; rostrum uti in praecedente sed brunneo-nigrum; pedes oculique sanguinolenti. Longitudo corporis $2^{\circ} 5^{\prime}$, caudae $1^{\circ} 1^{\prime}$, co alis $1.10^{\prime}$, rostri $1 \frac{1}{3}^{\prime}$; altiludo rostri $6^{\prime \prime}$, tarsi $3 \frac{x^{\prime}}{2}$.

Habitat prope Poçoens encima in sylvis Bahiae, ${ }_{3} \mathrm{Catingheira} \mathrm{ac} \mathrm{Ca}-$ puera" nominatis.

\section{Spequs 3 • PENELOPE JACUTINGA. TAв. LXX。}

Praecedente paulo minor, brunnescenti nigricans violaceoque renitens, absque splendore virescenti; crista capitis strigaque super oculos et versus latera colli albis, nigro striatis; tectricibus alarum mediis albis, apice nigro maculatis; fronte strigaque inframandibulari ac gulari super aures nigris; tectricibus juguli pectorisque albo marginatis; tarsis brevibus; paleari vix pendulo, rubicundo; gutture nigerrimo; remige extima, brevi, falcata, vix plumosa.

$\mathrm{D}$ ESCR I P I o. Corpus magnitudine fere praecedentis, supra-violaceo nigricanti splendens absque nitore virescenti, subtus totum fusco-violaceo nigrum; caput crista- 
tum, album, nigro striatum, plumis $1 \frac{1 /}{3}$ longis, basi medioque nigris, acuminatis, recumbentibus; fascia super apicem frontis, infra oculos et super aures nigra; regio circa oculos vix nuda, violaceo-nigra; striga super oculos et versus colli latera descendens alba, vix fusco variegata; mentum nigrum, lateraliter nigro strigilatum; capistrum nigro-plumosum; latera colli fusco-plumosa; jugulum superius nudum, flavo-rubicundum, medio vix carunculatum; nucha ac cervix immaculate nigricantes; plumae colli inferioris pectorisque nigro-fuscae, albo fimbriatae; abdomen, femora crissumque nigricantia, immaculata; tectrices humerales subviolaceo-nigricanti nitentes absque splendore viridi ac fimbriis albis, intermediae albae, apice nigro maculatae; remiges violaceo nitentes, nonnullae brunnescentes, extima brevis, falcata, tenuis, vix plumosa, subsequens longa, apice vix plumosa; cauda nigra, violaceo renitens; rostrum haud longum, non altum, basi nigrum, apice fusco brunnescens; pedes breviusculi, rubicundi. Longitudo corporis $2^{\circ} 3^{\prime}$, caudae $x^{\circ}$, c. alis 1 . $8^{\prime}$, rostri $1 \frac{1}{4}^{\prime}$, tarsi $2 \frac{3}{4}^{\prime}$.

Habitat in sylvis inter Bahiam et Rio de Janeiro, Penelopi pipili Jacq. similis; differt a Penelope cumanensi Jacq., a cl. Temminkio cum P. pipili unita, a cl. Merremio Leucolophos, et $\mathrm{Cujubi}$ ab Indis Parae nominata, quae non brunneo, sed tota nigra, violaceo viridique resplendens, crista capitis tola alba, regione circa oculos tota nuda, tectricibus humeralibus albo fimbriatis, juguio violaceo largeque carunculato etc. est; discrepat a Jacucaca crista capitis haud tota nigra, colore corporis non brunnescenti-viridi nitente.

\section{Speries 4. PENELOPE JACUPEBA. Taz. LXXi.}

Mediocris, supra brunnescenti-aeneo renitens; crista capitis fusco-aenea, immaculata; supercilio capistroque fuscis, albo strigilatis; pectore, abdumine, femoribus crissoque fusco-rufis; tectricibus juguli, pectoris, nec non humeri albo-fimbriatis; cauda supra violaceo-aenea, subtus livescente; remigibus brunneo-aeneis, scapis rufis,

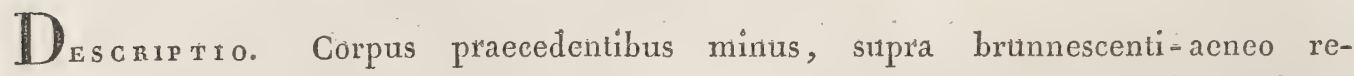
lucens; plumae capitis fuscae, haud acuminatae, $\mathbf{x}^{\prime}$ longae, albo non striatae; frons, supercilia, capistrum et cervicis latera fusca, albo strigilata; regio circa oculos et nares nuda, violaceo-nigra; gula ac guttur nudiuscula, flavo-rubicunda; nucha, dorsum uropygiumque brunneo-fusca, albo non maculata; tectrices juguli, pectoris, nec non humeri nigro-fuscae, aeneo relucentes, albo-fimbriatae; remiges fusco-brunnescenti viridique aeneo relucentes, scapis rufis, remiges extimae haud falcatae, plumosae; cauda nigricans, tombaceo relucens, subtus sulphurescenti-aeneo resplendens; 
rostrum haud altum, fusco-nigrum; pedes fusco-rubri, breves. Longitudo corporis $2^{\circ}$, caudae $10^{\prime}$, c. alis $1.6 \frac{1^{\prime}}{2}$, rostri $1 \frac{1}{2}^{\prime}, \operatorname{tarsi} 2 \frac{3}{4}$.

Habitat in sylvis Parae; differt a Jacúcaca colore non nigro sed tombaceo, cauda non tam longa, abdomine non nigricante, a Jacupemba pectore non cinereo et tectricibus alarum non rufo fimbriatis.

\section{Stecies. 5. PENELOPE JACUPEMBA. Taв. LXXII.}

Praecedente minor, fusco-aenea; crista capitis brevi, non albo-striata; capistro nigro; striga super ocilos rufescente; jugulo, pèctore, humerò nüchaque cirnerascentibus; tectricibus alarnm large rufo fimbriatis; abdomine, uropygio, crisso femoribusque rufis,

$\mathrm{D}$ Escriftio. Corpus magnitudine Phasiani picti, praecedentibus minus, supra virescenti-aeneo-fuscum, plumis basi rufescentibus, subtus cinerascens; pennae capitis breves, 7 "longae, erectiles, nigricanti-fuscae, albo non striatae; caput circa oculos, versus nares auresque nudum, nigro coerulescens; striga a fronte super nares versus occiput rufescens vel canescens; capistrum versus aures nigrum, albo non maculatum; mentum nigrum gulaque flavo-carnea, medioque longitudinaliter parum valvulosocarunculata, nuda; nucha tectricesque humeri virescenti-aeneae, plumis vix et marginibus modo cinerascentibus; jugulum pectusque cinerea, plumarum marginibus albo fimbriatis; tectrices alarum longiores uropygiique virescenti-aeneae, marginibus rufo fimbriatis; remiges virescenti-aeneo relucentes, margine externo fulvescente; abdomen crissumque rufa; femora rufescenti-fusca, plumis interdum rufo-marginatis; cauda subgradata, fusca, virescenti-aeneo sed non violaceo relucens, subtus livescenti atra; rostrum haud longum altumque, brunneo nigricans; pedes sanguineo-fusci; oculi castaneobrunnei. Longitudo corporis $2^{\circ}$, caudae $1^{\circ}$, c. alis $1.9^{\prime}$, rostri $1 \frac{1}{2}^{\prime}, \operatorname{tarsi} 2 \frac{3}{4}^{\prime}$.

Habitat in sylvis Rio de Janeiro, ad pagum indicum "Prezidio de St. Joâo", a cl. Temminkio Penelope superciliaris dicta; mas a foemina haud diversus.

\section{Stестёs. 6. +PENELOPE GUTTATA. Tав. LXXïl.}

Minor, brunnescenti vel tombaceosaenea, nec viridi resplendens, nec cinerascens; jugulo pectoreque brunneo-nigris, alho guttatis; abdomine pallide brunnescente; striga super oculos fulvescente nulla; dorso infimo non rufo sed brunnescente.

$\mathrm{D}$ ESCRiptio. Corpus Jacúpemba duplo minus, supra brunnescenti vel tombaceo renitens; caput fusco-aeneum, circa oculos, nares auresque nudum, rubicundo-vio- 
laceum, fronte et ad latera occipitis vix griseo-variegatum; cervix, dorsum, alae caudaque brunneo-rufescenti nitentia; gula nuda, rugosa, rubra, medio longitudinaliter vix nigricans; capistrum a basi mandibulae ad aures brunnescens; jugulum pectusque brunneo-fusca, albo nigroque guttata, cinereo non induta, plumis apice albo-terminatis; abdomen pallide brunnescens; crissum rufum; femora brunnea vel rufescentia; remiges brunneo renitentes, scapis nigricantibus; cauda gradata, brunnescenti-viridi aenea, rectricibus 3 extimis rufis, subtus rufescens; rostrum subsequente specie longius, aduncum, basi nigricans, apice albescens; pedes sanguiner; oculi brunnei. Longitudo corporis $1^{\circ} 4^{\prime}$, caudae $8 \frac{1}{9}^{\prime}$, c. alis $1.6 \frac{1}{5}^{\prime}$, rostri $1^{\prime}$, tarsi $2^{\prime}$.

Habitat super arbusta ad flumen Solimoëns, Araucaun vociferans agnominata.

\section{Sígries 7. + PENELOPE ARAUCUAN. Ta . LXXIV.}

Pallide brunneo-aenea, precedenti similis colore sed virescens; jugulo, pectore tectricibusque humeralibus non cinerascentibus; abdomine pallide albescente; capite rufescente; dorso infimo castaneo; striga super oculos fulvescente.

D. EScriptio. Corpus praecedente vix maius, supra pallide brunnescens, tombaceo-virescenti relucens; caput rufescens, circa oculos, nares auresque nudum, violaceorubrum, fronte albicante, striga super oculos rufo-fulvescente; gula nuda, rubicunda, medio longitudinaliter nigra; jugulum, pectus et in mare tectrices alarum cinerascentes, plumis basi fusco - brunneis, apice cinereis; tectrices dorsi anterioris in mare apice aeque cinereo-fimbriatae, in foemina vix; dorsum infimum maris castaneum, foeminae rufescens; remiges brunneo-aeneae, vexillo externo canescente; cauda brunnescenti-viridi relucens, rectricibus 3 exterioribus basi fusco-virescentibus, apice rufis; abdomen sordide albicans; femora pallide brunnescentia ; crissum rufescens; rostrum breviusculum, nigricans, apice vix albescens; pedes nigricantes, vix sanguinei.

Habitat in lacis aquosis Provinciae Maranhao ad flumen Itapicuru et in sylvis ad pagum St. Domingo districtus Minas Novas; differt a praecedente pectore cinerascente abdomineque albescente.

\section{Familia: Columbae.}

In sylvis et campis supra arbores nidum e ligno construentes, familiares, monogamae, volatui haud ineptae, granivorae; rostro tenui, rectiusculo, non alto, medio compresso ac tenuiore, apice tumido, declivi, non dentato, acuto; naribus mediis, squama tectis; tarsis digito medio haud longioribus; digitis liberis, postico terrae incumbente. 
Magnitudine passeris.

\section{Species 1. COLUMIBINA STREPITANS. Tad. LXXY. i.}

Supra cinerascens, subtus et in frontis apice albicans; capite plumbeo; pectore fulvescente; remigibus nigris; tectricibus alarum albis, supra humerum violaceo 1-fasciatis; rectricibus longiusculis, 3 extimis albis, intermediis cinereis.

$\mathrm{D}$ E S C r p т 0 . Corpus Merula fere duplo minus, supra brunnescenti-cinereum, subtus album, fulvescenti nebulatum, plumis basi albis; caput nuchaque plumbescentia, fronte alba; gula alba; jugulum pectusque alba, rosaceo nebulata; abdomen, crissum femoraque alba; tectrices dorsi, uropygii et scapulares cinereo brunnescentes, immaculatae; tectrices alarum mediae albae, fascia pone humerum violaceo-nitente, axillares nigrae; remiges primariae fusco-nigrae, basi albae, secundariae subjacentes nigrae, apice albomarginatae, superjacentes cinereo brunnescentes, margine externo albescente; alae subtus antice nigerrimae, postice albicantes; cauda gradata, longiuscula, supra cinereo-brunnescens, subtus tota alba, rectricibus 2 intermediis brunnescentibus, reliquis, praecipue exterioribus totis albis; rostrum gracile, breve, nigrum, subtus basi flavicans; pedes flavo-rosacei. Longitudo maris $6 \frac{x^{\prime}}{2}$, caudae $3^{\prime}$, c. alis $1.2^{\prime}$, rostri $7^{\prime \prime}$, tarsi $5^{\prime \prime}$.

Habitat in campis $\mathrm{Pia}$ uhy, mas foemina maior, alis Crotali horridi instar crepitans, ideoque ab incolis Cascavelha, ab Azara Picui nominata.

\section{Steques 2. ${ }_{4}$ COLUIMBINA CAIMPESTRIS. Taв. LXXV. 2.}

IMinor, supra immaculate brunnea, plumis basi cinereis; fronte plumbea; gula juguloque pallide rosaceis; abdomine albo; remigibus brunneofuscis; maculis alarum 5 violaceo-nigris, anterioribus albo terminatis; rectricibus intermediis brunneis, reliquis nigris, 3 extimis apice allbis; rostro minuto.

$\mathrm{D}$ ESCR I T то. Corpus praecedente minus crassum, supra brunneum, immaculatum; caput brunneum, fronte plumbea; gula jugulumque brunnescenti-rosaceo nebulata; abdomen, crissum femoraque alba; nucha, dorsum, uropygium rectricesque intermediae brunnea; tectrices alarum brunneae, longitudinaliter albo 2 , nigro 5 maculatae; remiges fusco-brunneae, basi subtus albae; alae sub humero fuscescentes; cauda longiuscula, gradata, rectricibus subjacentibus nigerrimis, 3 extimis apice, praecipue rexillo externo albis; rostrum minutum, nigrum; pedes flavi; oculi albescentes. Longitudo maris $6 \frac{3^{\prime}}{4}$, caudae $3 \frac{1}{2}^{\prime}$, c. alis $1.3^{\prime}$, rostri $5^{\prime \prime}$, tarsi $7^{\prime \prime}$. mare minor.

Habitat gregaria in campis Bahiae, "Cabóclas" ab incolis denominata; foemina 


\section{Speres 3. COLUMiNA CABOCOLO. Taв. LXXva. Mas. 1.}

Majuscula, brevicaudata, supra subtusque cinammomea; capite plumbeo; remigibus nigris; alis supra 8-9) nigro strigilatis; cauda breviuscula, aequali, supra cinammomea, subtus nigerrima.

$\mathrm{D}$ Escripto. Corpus praecedente vix maius, supra cinammomeum, sublus cinamomeum violaceo vix nebulatum; caput plumbescens; mentum albicans; pectus cinammomeum, violaceo vix indutum; abdomen, crissum, femora, uropygium, dorsum tectricesque alarum cinammomea; maculae 8 - 9 nigrae, super alas dispersae; remiges tectricesque axillares nigrae; alae subtus nigrae; cauda brevis, aequalis, supra cinammomea, subtus nigra, rectricibus intermedis cinammomeis, reliquis nigris, apice cinammoneo fimbriatis; rostrum tenue, nigricans, apice intumescens; pedes flavi, extrorsum hirsuti. Longitudo maris $6 \frac{1^{\prime}}{2}$, caudae $1 \frac{x^{\prime}}{2}$, c. alis $1.1^{\frac{1}{2}}$, rostri $6{ }^{\prime \prime}$, tarsi $7^{\prime \prime}$; foemina mare vix minor colore brunneo-cinammomeo.

\section{Speres. 4. COLUIMBINA GRISEOLA. Taв. LXXVa. 2.}

Minima, brinnescenti-grisea; plumis capitis pectorisque squamosis; remigibus rufis, apice margineque externo fuscis; cauda aequali, hrevi, supra brunneo-cinerea, subtus nigra, rectrice extima apice albo marginata; tectricibus alarum cinerascentibus, albo terminatis.

$\mathrm{D}$ escriptio. Corpus praecedentibus longe minus, supra brunneo-griseum, subtus brunneorcinereum; caput pectusque brunnescentia, plumis squamatis; nucha, dorsum uropygiumque cinereo-brunnea; mentum, nec non abdomen crissumque sordide albicantia; hypochondria pallide brunnescentia; tectrices alarum cinerascentes, albo terminatae, maculis supra humerum nonnullis sanguineis; tectrices axillares rufescentes; remiges rufae, vexillo externo apiceque fusco-rufo marginato; alae subtus rufae; cauda supra cinerascens, subtus nigra, rectrice extima apice albo-marginata; rostrum breve, nigricans; pedes flavi; oculi grisei. Longitudo corporis $5^{\prime}$, caudae $2^{\prime}$, c. alis 1. $1 \frac{x^{\prime}}{4}$, rostri $6^{\prime \prime}$, tarsi $6^{\prime \prime}$.

Habitat in sylvis fl. A mazonum.

\section{Familia: Perdices.}

Pedestres, brevicaudatae, volatui alto ineptae, agrestes ac campestres, cursoriae, familiares, in terra nidificantes, granivorae, frequenter semipalmatae; cauda brevissima; digito postico breviusculó, plus minusve elevato; periophthalmiis nudiusculis; rostro subarcuato, non dentato, in nonnullis brevi, compresso, vel cylindrico, vel longiore, tenui ac columbino; naribus patulis aut squama aut membrana tectis; tarsis plerumque haud altis. 
Genibus plumosis.

\section{Genus 1. P E R D I X.}

Semipalmata; capite circa oculos nudo; digito postico terrae subincumbente; pedibis haud hirsutis; femoribus usque infra genua plumosis; cauda rotunda, subconspicua; rostro brevius= culo, subcylindrico, apice curvo; naribus plerumque squama tectis.

\section{Stecies 1。PERDIX GaPUEIRA.Tá LXXYif}

IVlediocris, sylvestris, supra brunnescens, plumis nigre-maculatis rufoque transversim lineatis strigisque nona nullis albis, subtus cinerea; rostro compresso, alto; stria super frontem et oculos rufescente; dorso infimo rufescenti-fulvo, nigro strigilato; remigibus albo 6 fasciatis vel guttatis.

$\mathrm{D}$ escriptio. Corpus Perdice cinerea vix minus, rufescenti-brunneum, maculis nigris strigisque rufis nigrisque transversis variegatum, subtus plumbescenti-cinereum; caput brevi-cristatum, brunneo-rufum, circa oculos et nares nudum, fronte striaque fulvopunctata super oculos rufescentibus; lora, gula, pectus abdomenque cinerea; femora cinerea, infra brunnescentia; crissum rufo-brunneum; dorsum superius tectricesque alarum brunneo - nigro rufoque varia, plumis medio albo-substrigilatis, versus apicem nigro rufoque transversim strigilatis nigroque large maculatis, apice haud raro nigro rufoque lineolatis, in tectricibus axillaribus albo guttatis; dorsum infimum fulvescens vel rufescens, maculis nigris dispersis; remiges primariae nigrae, vexillis externis albo 6 guttatis, secundariae brunneo-rufescentes, fulvo obscure fasciolatae nigroque lineolatae; alae subtus cinereae, antice brunneo variae; uropygium brunnescens, transversim nigro lineolatum; cauda brevis, $I^{\prime}$ conspicua, nigricans, !transversim rufo lineolata; rostrum breve, aquilinum, compressum, altum, nigrum, naribus basalibus mernbrana tectis; pedes nigricantes, vix flavicantes, digito postico longiusculo, terrae incumbente. Longitudo corporis foeminae $10^{\prime}$, caudae $2 \frac{1^{\prime}}{2}$, rostri $9^{\prime \prime}$; altitudo rostri $7^{\prime \prime}$, tảrsi $1 \frac{3}{4}^{\prime}$.

Habitat in sylvis Rio de Janeiro et Minas Geraës proximis, vesperam nec non noctem cantu gallinaceo indicans; foemina mare minor, ab incolis Capue ir a vel Caproh, ab authoribus Perdix guianensis et dentata dicta. 
60

\section{Species 2. PERDIX RUFINA. Tab. LXXY? a}

Sylvestris, praecedente vix minor, supra subtusque rufa; capite rufo nigroque fasciolato; nucha cinerascente; loris rufis; rostro haud alto.

Descrítio. Corpus praecedente vix minus, supra brunneum, nigro parum macutatum et lineatum, subtus rufum; caput subcristatum, rufo nigroque fasciolatum, supra aures fulvescens, loris rufs; mentum vix rufum; gula nuchaque cinerascentes, albo transversim sublineolatae; pectus rufum, fulvo transversim undulatum; femora rufobrunnea; tectrices dorsi anterioris longioresque alarum rufescentes, nigro transversim lineolatae, apice large nigro et fulvo vel rufo fasciatae, dorsi medii et posterioris uropygiique breves, fulvescentes, nigro punclulatae; remiges primariae fusco-brunnescentes, vexillo externo 8 - 9 fulvo-maculato, secundariae fulvo subfasciatae nigroque lineolatae; cauda rotunda, rufo-brunnescens, supra subtusque fulvo nigroque lineolata, $2^{\prime}$ conspicua; rostrum breve, minus altum ac gibbum, brunneo-fuscum, naribus patulis; pedes fusco-flavi. Longitudo corporis 8 ', caudae $2 \frac{{ }^{\prime \prime}}{2}$, rostri 9 "; altitudo rostri $5 \frac{1}{2}{ }^{\prime \prime}$, tarsi $1 \frac{\pi}{2}$.

Habitat in sylvis fl. A m a z on um, solitarie humi incedens.

$$
\text { Genibus haud plumosis. }
$$

Rictu oris usque infra oculos prolongato; collo elongato; digitis haud semipalmatis; cauda vix conspicua, rectricibus rigidis fere nullis.

\section{GENUS2. RHYNCHOTUS.}

Rostro elongato, corneo, haud alto, subarcuato, apice convexo, acuto, nec curvo, nec lateraliter sulcato; naribus fere basalibus; digito postico elevato, breviusculo; genibus nudis; tarsis antice scutato-fasciatis, postice reticulatis.

\section{Stecies 1. RHYNCHOTUS FASCIATUS. Tá. LXXVIF.}

Maior, supra brunnescens, fulvo nigroque fasciata; collo toto, remigibus pectoreque rufis; abdomine, femoribus caudaque cinerascentibus, nigro fasciolatis; gula alba; capite rufo, nigro-striato.

Descripto. Corpus Gallina domestica minus, brunneo-rufum; eaput rufum, medio nigro striatum, circa oculos vix nudum, genis lorisque ochraceis, macula exigua super aures 
nigricanti; gula alba; collum supra subtusque et pectus castaneo-rufa; dorsum, tectrices scapulares et humerales, remiges secundariae superjacentes caudaque pallide brunnescentia, nigro fulvoque fasciata, plumis apice fulvo marginatis; tectrices axillares remigesque primariae immaculate castaneae, secundariae subjacentes castaneae, nigro fasciatae, scapis nigris; alae subtus castaneae; abdomen maris fulvescens, immaculatum, foeminae aeque ac femora, cauda sublus crissumque brunnescenti-cinerea, nigrofasciolata; rostrum corneum, elongatum, apice acutum; pedes supra genua nudi, versus digitos crassiores, pallide flavicantes, digitis subfimbriatis; oculi albo-flavi. Longitudo corporis foeminae $1^{\circ} 3^{\prime}$ caudae $2^{\prime}$, c. alis $1_{1} 1^{\prime}$, rostri $2^{\prime}$; altitudo rostri $4^{\prime \prime}$, tarsi $2^{\prime}$.

Habitat, „En a pu pê z' dictus, inter Promelias in campis Provinciarum St. Pa uli et $\mathrm{M}$ inas Ge-raës; mas multo minor, abdomine pallide fulvesenti, foemina maculis dorsalibus nigris interdum latioribus.

\section{GENUS 2。 P E Z U S.}

In sylvis campestribus vivens; rostro haud elongato, lateraliter sulcato, apice subintumescenti, deflexo; naribus intermediis; digitis breviusculis, postico elato, unguiculato absque phalangibus; tarsis antice et postice scutellato-fasciatis.

\section{SPECIES 1。 PEZUS SERRATUS. TAB: LXXVi。}

Praecedente maior, supra cinammomeo-fuscus, nigro undulatus, subtus colore Capreoli; gula alba; crisso femoribusque rufo nigroque fasciatis; capite fusco-sanguineo; tarsis postice scabris.

$\mathrm{D}$ ESCR I P I o. Corpus robustum, magnitudine fere Gallinae domesticae, supra ci= nammomeo-fuscum, relucens, undulate nigro-fasciatum; caput fusco-sanguineum, circa oculos nudum; plumae colli breves, rufescentes, nigro fasciolatae et quasi exesae; gula alba; pectus brunnescens, praecipue abdomen colore capreoli; femora fulva, nigro-brunneo fasciata; crissum rufescens, nigro - fusco maculatum sive fasciatum; tectrices alarum olivaceo-brunneae, nigro fasciolatae, axillares rufae, nigro lineolatae; remiges secundariae rufae, nigro fasciatae, vexillo interno fusco, vix nigro fasciato, primariae fuscescenti brunneae, immaculatae; axillae subtus brunneae, nigro lineolatae; alae subius cinerascentes; cauda minuta, brunneo-fusca, ferrugineo nigroque vix fasciolata; rostrum nigro-fuscum, subtus flavicans; pedes nigri, postice subrèticulati, asperi, subserrati, digitis brevibus, unguibus brevibus, rotundatis, latiusculis. Longitudo corporis $1{ }^{\circ} 4 \frac{x^{\prime}}{2}$, caudae $2^{\prime}$, rostri $1^{\prime} \frac{I}{3}^{\prime}$, tarsi $2 \frac{I}{4}^{\prime}$.

Habitat in sylyis campestribus $\mathrm{Al} . \mathrm{N}$ ig ri. 
Majusculus, subtus rufus; periophthalmiis, gula abdomineque ferrugineis; jugulo_colloque cinereis; pectore castaneo; dorso, alis caudaque ferrugineo nigroque aéque fasciatis.

$\mathrm{D}$ Ecriptro. Corpus Columba domestica minus, subtus rufum; caput nigricans, vix rufescens, circa oculos, aures gulamque rufescenti ferrugineum; jugulum et cervix cinammomeo-fusca, plumis juguli nigro fasciolatis; pectus superius nuchaque inferior brunneofusca, cinereo nebulata; pectus inferius castaneum; abdomen ochraceum; femora, hypochondria crissumque feriuginea, nigro fasciata; dorsum anterius fusco - cinammomeum, non fasciatum, reliquum rufo nigroque aeque fasciatum; tectrices alarum omnes, remiges secundariae suprajacentes, reliquae vexillo externo, caudaque rufescenti-ferrugineo nigroque fasciatae; remiges primariae nigro-fuscae, haud fasciatae, vexillo externo brunnescenti; axillae nigricantes; alae subtus cinereo-albescentes; rostrum supra fuscum, subtus flavicans; pedes altiores quam in Tinamo variegato Buff. digitique crassiores, flavi, unguibus brevibus, rotundioribus. Longitudo corporis $1^{\circ} \frac{1}{2}^{\prime}$, caudae $2 \frac{1}{2}^{\prime}$, c. alis $1.1^{\prime}$, rostri $1 \frac{1}{2}^{\prime}$, tarsi $2^{\prime}$.

Habitat pedestris et haud fugitivus in limite sylvarum campestrium (Catingha), ab incolis $\mathrm{Z} a b$ el é nominatus; differt a Tinamo variegato $\mathrm{L}$ ath. corpore maiore, collo non castaneo, periophthalmiis non nigris, fasciis alarum dorsique nigris, non latis sed angustis, digitisque non tenuibus.

\section{SPecies 3. PEZUS YAPURA. Tab. LXXVIII.}

IMajusculus, supra et in collo brunneo-fuscus, nigro tenerrime transversim lineolatus, subtus cinerascens; crisso ferrugineo nigroque fasciato; femoribus fuscescentibus, immaculatis;

$\mathrm{D}$ ES R r ptro. Corpus Columba domestica maius, supra brunneofuscum, subtus cinerascens; caput nigricans; gula alba; collum, dorsum, uropygium, cauda, tectricesque alarum brunneo-fusca, transversim nigro tenerrime lineolata; axillae remigesque nigrofuscae, vexillo externo brunnescentes, non fasciatae, secundariae superjacentes clarius brunnescentes, praecipue vexillo externo transversim nigro lineolato; alae subtus cinereae; latera pectoris abdominisque et femora brunnescenti-fusca, non fasciata; crissum ferrugineo nigroque fasciatum; rostrum nigrum; pedes flavi, unguibus non rotundis; oculi brunnei. Longitudo corporis $11^{\prime}$, caudae $2^{\prime}$, c. alis $1.1^{\prime}$, rostri $1 \frac{3}{4}$, tarsi $2^{\prime}$.

Habitat in sylvis fl. Japurae et Solimoëns, ab Indis In ar ubú dictus; foemina femore ferrugineo nigroque fasciolato. 


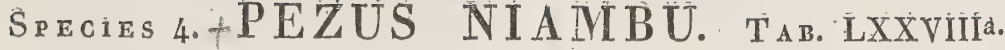

Minor, cinammomeo-fuscus supra, subtus cinereus; capite nigerrimo; crisso fülvo, nigro-maculato; petç tore nigricanti.

$\mathrm{D}$

ESCRIPTIO. Corpus Tiname Tataupa Swainson maius, supra saturate cinammoneum, subtus plumbescens; caput nigerrimum, pone aures cinerascens; gula alba; collum pectusque nigricanti-plumbea; abdomen pallide cinerascens; latera femorum et circa anum nigra, albo variegata; crissum fulvum, nigro variegatum; femora infra fusca; dorsum, uropygium caudaque fusco-cinammomea vel castanea; tectrices alarum vexillumque remigum secundariarum superjacentium externum castaneo-brunnea, apice obscure nigro-lineolata; tectrices axillares remigesque primariae non brunneae sed nigro-fuscae; alae subtus cinerascentes; rostrum rubicundum, apice nigricans; pedes et oculi rubicundi; tarsi digitiqué longiores quam in Tinamo Tataupa. Longitudo corporis $8^{\prime}$, caudae $2^{\prime}$, c. alis $1.1 \frac{1}{2}^{\prime}$, rostri $1 \frac{1^{\prime}}{3}$, tarsi $1 \frac{1}{2}^{\prime}$.

Habitat in campis districtus adamantini et Bahiae, $\mathrm{Niambú} \mathrm{Ch}$ or oro appellatus: foemina paulo minor, capite cinerascenti, dorso minus fusco; victitat seminibus; differt a Tataupa voce, corpore maiore, colore supra non brunneo sed fusco-castaneo, capite non cilereo sed nigro, pectoreque nigricanti.

\section{GENUS. T I N A I U S}

Campestris, coturnici similis; rostro brevi, lateraliter sulcato, apice unguículato, ḋeflexo; näaribus subbasalibus, squama tectis; digito postico elevato, brevi; unguibus nön rotundis; catido, cuneata, deflexa; rectricibus nullis

\section{Species 1。TINAMUS BORAQUIRA. Tas. LXXIX:}

IMajor, albescens, alboque strigilatus, supra dorsum brunneus, nigro fasciatus et lineolatus; gula, abdomine femoribusque albis; jugulo fusco-maculato; pectore, hypochondriis tectricibusquie alärum, praecipue posterioribus allicantibus, nigro-undulatis,

$\mathrm{D}$ ESCRIPTIO. Corpus subsequentibus paulo maius, non rufescens sed albescens; albostrigilatum; caput medium nigricans, plumis albo marginatis, fronte, nec non lateribus occipitis et auribus rufescentibus, genis vix plumosis; gula, abdomen; femora crissurnque alba; cervix vix rufescens; nucha nigro - fusco striata; jugulum albescens, nigro-fusco lineolata; tectrices dorsi brunneae nigroque maculatae, vel potius ni- 
gro fasciatae et undulatae, lateraliter albo strigilatae; tectrices humerales et axillares fulvo albescentes, fusco-nigro fasciolatae, posteriores magis albicantes nigroque transversim lineolatae; remiges primariae nigricantes, vexillis externis fulvo $11-12$ guttatis, internis immaculatis, secundariae fulvescentes, nigro-fusco fasciatae; alae subtus antice fulvo nigroque variegatae, postice cinereo-fuscae; cauda brevis, pallide fulvo-maculata vel fasciata nigroque undulata, rectricibus nullis; rostrum breve, supra brunneo-fuscum, subtus flavicans; pedes circulariter scutellati, flavi. Longitudo corporis $99^{\prime \prime}$, caudae 2', . c. alis $1.1^{\prime}$, rostri $9^{\prime}$, tarsi $1 \frac{1}{3}^{\prime}$.

Habitat in campis petrosis districtus adamantini; persecutus antra petrosa petit.

\section{Stecies 2. TINA IMUS MAIOR. Tab. LXXX.}

Maior, non albescens sed rufescenti-brunneus, subtus ferrugineus; nucha, jugulo pectoreque nigro alboque strigilatis; tectricibus humeralibus rufis, nigro non fasciatis sed guttatis, dorsalibus caudalibusque brunneis, nigro non fasciatis sed maculatis alboque limbatis; alis subtus rufis.

$\mathrm{D}$ ESCRítio. Corpus praecedente vix minus, super rufescenti-brunneum nigroque maculatum, nec non albo strigilatum, subtus ferrugineum; caput rufo-brunnescens, nigro fulvoque maculatum; gula alba; cervix rufescens; collum supra et subtus, jugulum pectusque brunneo-rufa, nigro pallideque fulvo strigilata; abdomen femoraque rufoferruginea; hypochondria crissumque ferruginea, nigro fasciolata ; tectrices dorsi brunneae, medio haud variae sed nigro maculatae, et lateraliter fulvo-albo limbatae, alares rufae, nigro guttatae; remiges secundariae rufescentes nigroque fasciatae; primariae nigricantes, vexillis externis fulvo fuscoque 12 guttatis, internis obscure fasciatis; alae subtus antice ferrugineae, postice nigro rufoque fasciatae; cauda olivaceo-brúnnea, nigro maculata fulvoque lineata; rostrum breve, brunnescens, subtus flavicans; pedes oblique scutellati, flavi, altiores. Longitudo corporis $9^{\prime}$, caudae $1 \frac{3}{4}{ }^{\prime}$, c. alis $1.1 \frac{x^{\prime}}{2}$, rostri $9^{\prime \prime}, \operatorname{tarsi} 1 \frac{2}{3}^{\prime}$.

Habitat in campis Minas Geraës prope pagos Tejuco et Contendas. 


\section{Species 3. TINAMUS MEDIUS. Tá. LXXXt.}

Praecedente minor, supra rufescens, nigro fasciolatus alboque strigilatus, subtus ferrugineus; pectore ferirugineo, nigro-guttato; tectricibus alarum nigro fasciolatis; rostro longiore.

D Es criptio. Corpus praecedente paulo minus, supra rufo-brunneo nigroque fasciolatum fulvoque strigilatum, subtus ferrugineum, jugulo pectoreque nigro-gultulatis; caput brunneum, nigro nec non albo submaculatum, super oculos rufescens; gula alba; nucha rufescens, vix nigro striata; tectrices dorsales cinerascenti-brunneae, medio rufo nigroque fasciolatae, lateraliter fulvo-albo strigilatae, alares rufescentes, nigro transversim undulatae; remiges rufae, nigro fasciatae, primariae nigro-fuscae, vexillis externis fuivo guttatis, internis rufo fasciatis; alae subtus antice ferrugineae, postice rufae, nigro fasciatae; cauda fulva, nigro fasciolata; hypochondria crissumque ferruginea, nigro fasciolata; abdomen femoraque immaculate rufescentia; rostrum paulo longius, quam specie praecedente fuscum, subtus flavicans; pedes digitique robusti, flavicantes, ungue medio triquetro. Longitudo corporis $8^{\prime}$, caudae $\mathbf{1}^{\prime}$, c. alis $1 . \frac{1}{4}$, rostri $\mathbf{1}^{\prime}$, tarsi $1 \frac{1^{\prime}}{3}$.

Habitat in campis prope pagum Tejuco; an foemina praecedentis, dorso licet tectricibusque humeri diversis?

\section{Species 4. TINA TIUS MINOR. Tab. LXXXiI.}

IVinimus, supra rufus, nigro sublituratus alboque substrigilatus, subtus ferrugineus; capite rufo, nigro. liturato; tectricibus alarum nigro tenerrime fasciolatis.

$\mathrm{D}$

ESCr1p т о. Corpus praecedentibus minus, rufum, striis albis raris, subtus. ferrugineum; caput rufum, nigro punctulatum, albo non maculatum; gula alba; colluin jugulumque ferruginea, brunneo substrigilata; pectus, abdomen femoraque immaculate ferruginea, hypochondriis crissoque tenerrime fusco-fasciolatis; tectrices dorsi caudaeque rufae, nigro tenerrime angulato-fasciatae vel lituratae, tectrices alarum rufae, nigro fasciolatae; remiges primariae nigricantes, vexillis externis fulvo-rufo guttatis, internis rufo nigroque fasciatis; alae subtus ferrugineae, postice rufo nigroque fasciatae; cauda fere nulla; rostrum minutum, brunneum, subtus flavicans; pedes breviusculi, circulariter scutellati, flavi, digitis brevibus. Longitudo corporis $7^{\prime}$, rôstrí $7^{\prime \prime}$, tarsi $1^{\prime}$.

Habitat in campis prope pagum. Tejuco, Cadornix nominatus. 


\section{Familia: $\mathrm{O}$ ti d e s.}

Aves torrestres, avibus Grallis vel amphibiis corporis structura affines, stentorosae et longisonae, omnivo. rae, frequenter cursores, sylvaticae vel campestres, ponderosae, excepto genere Otidis 3 -dactylo 4 dactylae, subsemipalmatae; pedibus altis, super genua large nudis, digito postico frequenter vix incumbente; axillis tuberculatis, in sola Palamedea spinosis; rostro-subgallinaceo, compresso, subarcuato; naribus transversis. (Genera: Otis, Dicholopus, Falco serpentarius, Psophia, Palamedea.)

\section{GENUS 1. P S O P H I A.}

Campestris, alatui inepta, ardearum instar incedens, gallinarum modo mansuefacienda, alas frequenter conculiens; voce valida, profunda, gutturosa; capite tomentoso, inermi, vix circa oculos nudo; rostro conico, magis alto quam lato, compresso, subarcuato; naribus mediis; axillis tuberculatis; collo elongato; pedibus altis, supra genua nudis; digitis basi parum subpalmatis, postico breviusculo, vix incumbente; cauda fere nulla.

\section{Siecies. 1. PSOPHIA VIRIDIS. Tab. LXXXIII.}

Maior; supra viridis, subtus nigra; tectricibus dorsi remigibusque secundariis nec albis nec cinereis sed viridibus, nec non ferrugineis; tectricibus humeri virescenti-, mediis alarum coeruleo-nitentibus; jugulo infimo violaceo-splendente.

$\mathrm{D}$ Escriptio. Corpus Psophia crepitante fere minus, supra dorsum scapulasque viride, ferrugineo immixto, subtus nigerrimum; caput collumque nigra, tomentosa; collum infimum jugulumque violaceo et vix viridi splendentia; tectrices dorsales scapulalaresque saturate virides, nec non ferrugineo mixtae, setosae, longiusculae, basi nigrae; tectrices alarum intermediae nigrae, apice partim viridi, partim coeruleo splendentes; remiges primariae secundariaeque nigrae, secundariae suprajacentes brunneofuscae, vexillo externo brunnescenti ac virescenti; axillae alaeque subtus nigrae; cauda brevissima, alis minor, nigra; gula, jugulum, pectus, abdomen, hypochondria, femora crissumque nigra; femora dimmidia infra nuda; tarsi alti, scutellato-fasciati, olivaceo-argentei; digiti breviores quam in speciebus reliquis, vix semipalmati; rostrum conicum, albescens, basi vix plumbescens. Longitudo corporis $1^{\circ}, 3 \frac{3{ }^{\prime}}{}{ }^{\prime}$, caudae $3 \frac{{ }^{\prime}}{2}$, rostri $1 \frac{x^{\prime}}{2}, \operatorname{tarsi} 4 \frac{\mathrm{I}^{\prime}}{3}$.

Habitat in campis sylvestribus fl. Amazonum ad pagum Villa Nu ora. 


\section{Species 2. PSOPHIA LEUCOPTERA. Tab. LXXXXiv.}

Maxima, supra subtusque nigra; remigibus secundarïs albissimis; tectricibus alarum intermediis violaceo ac viridi nitentibus; dorso nigro; collo infimo juguloque cupreo-violaceis.

D

E SCR If Tro. Corpus reliquis speciebus paulo maius, supra subtusque nigerrimum; caput collumque nigra, tomentosa, periophthalmiis nudiusculis; collum infimum cupreom violaceo renitens; gula, pectus, abdomen, femora seminuda, crissum, tectrices dorsales, humerales, axillares et scapulares, remiges primariae caudaque nigerrima; tectrices alarum intermediae basi nigrae, apice violaceo nec nón viridi splendentes, posteriores longae remigesque secundariae basi nigrae, versus apicem albae, secundariis nonnullis subjacentibus albo nigroque variegatis; alae subtus nigrae; cauda brevis, alis vix maior, nigra; pedes alti, albo-olivacei; digiti basi modo subpalmati, unguibus compressis, falcatis, nigris; rostrum subconicum, subarcuatum, albescens; oculi brunnei. Longitudo corporis $1^{\circ}, 5^{\prime}$, caudae $4^{\prime}$, rostri $1 \frac{3}{4}^{\prime}, \operatorname{tarsi} 5 \frac{1}{2}^{\prime}$.

Habitat, carne victitans, gregaria in sylvis campestribus ad $\mathrm{fl}$ R io $\mathrm{Negro}$, nec non in cortibus mansuefacta; mas a foemina non distinctus. 
68.

\section{Aves Grallae s. Amphibiae.}

Tetra-, raro tri-dactylae, litorales et palustres, haud raro in arboribus nidificantes, plerumque semipalmatae, amphibiis, insectis aquaticis piscibusque victitantes; tarsis, nec non collo frequenter altissimis; femoribus seminudis.

\section{Familia: I b i d e s.}

Tetradactylae, semipalmatae, in locis paludosis fluminum nec non sylvarum haud raro gregarie aut solitarie victitantes, arhoribus insidentes vel in sylvis gregarie terra incedentes et vociferantes; rostro longo, haud crasso, arcuato et deorsum falcato, cylindrico, maxilla inferiore compressa, margine oris introrsum connivente; facie circa frontem, oculos gulamque nuda; naribus basalibus; collo subelongato; digito postico longo, incumbente; ungue digiti medii non pectinato; tarsis reticulatis.

\section{Genus 1. T A N T A U S.}

Statura fere Ciconiae; tarsis altis, digito medio duplo longioribus; rostro basi crassiore et altiore, apice acuto, dentato; naribus osseis, membrana non tectis.

\section{Species 1. TANTALUS PLUMICOLLIS. Tab. LXXXY.}

Magnus, alhus, ochraceo vix indutus; collo fuscescenti-plumoso; occipite nuchaque nigricantibus; remigibus primariis caudaque violaceo-nigris; rostro flavescenti, supra frontem impresso; genis gulaque nudis, rubicundis; pedibus flavicanti-nigris.

D E Criptio. Corpus Ciconia europaea haud minus, supra subtusque album, collo tectricibusque dorsi anterioris vix rufescentibus; caput crassum, in fronte et vertice, supra aures, circa oculos, basin maxillae genasque nudum, rubicundum, loris parum alboplumosis, striga circa aures fuscescente; occiput nuchaque nigricantia, plumis basi albis; collum superius fusco-albescens, inferius album, ochraceo nebulatum; tectrices dorsi, uropygii, abdominis, femorum, crissi, axillarum alarumque albae; remiges primariae tectricesque alarum superaxillares caudaque nigrae, violaceo renitentes, secundariae superjacentes albae, nonnullis fuscis immixtis; alae subtus antice albae; cauda alis fere brevior; rostrum flavescens, longum, apice arcuatum, basi crassius, naribus subsuperis, osseis, ellipticis. Longitudo corporis $2^{\circ}, 7^{\prime}$, caudae $7^{\prime}$, rostri $7^{\prime}$, pedum $2^{\circ}, 1^{\prime}$.

Habitat in insula St. Joannis sive Marajo, nec non ad flumen St. Francisci; non differt a Tantalo Ibide aegyptiaco vel senegalensi, ex imagine picta modo mihi cognito, 
è Brasilia a me jam allato nisi rostro ḋigitisque longioribus, rostro basi altiorè, remigibus catidaque nonnisi violaceis occipiteque nigricante, a Tantalo Loculatore vero, Mulecco ab incolis nominato, corpore maiore alboque, cuius rostrum longius ac basi nigrum, apice virescens, collüm in juvenibus albo-plutiosum, in adultis nudum, nigrum squamisque albis tectum, caput denique totum nudum.

\section{GENUS 2. I B I S.}

Minor; rostro basi vix crassiore, magis arcuato, apice obtusiusculo, non déntate; naribus membrana tectis; collo subetongato, curvato; tarsis haud altis.

\section{Stecies 1。 IBIS NUDIFRONS. Tab. LXXXVI。}

IMínor, "nigra, violacéo cupreoque-viridi relucens; fronte tota, periophthalmiis, genis, gula rostroquie nudis, luteis; pedibus rubris; nucha non cristata.

D

E S C r I T т o. Corpus Ibide cayennensi minus, supra subtusque nigrum, alis caudaque virescenti violaceoque chalybeis, plumis basi nigris; caput nigrum, in fronte tota, circa oculos, gulam basinque rostri nudum, luteum; nucha non cristata, nigra; remiges primariae magis virescentes, secundariae breviores, magis cupreo-chalybeae; alae sub= tus nigrae; cauda aequalis, alis haud longior; rostrum tenue, cylindricum, luteum, margine haud dentatum, naribus exiguis, ovatis, sulcatis; pedes breves; rubicundi. Longitudo corporis $1^{\circ}, 8^{\prime}$, caudae $4 \frac{3}{4}^{\prime}$, rostri $4 \frac{3}{4}^{\prime}$, pedum $5^{\prime}$, tarsi $2 \frac{1}{4}^{\prime}$.

Reperitur solitaria ad litora lacuum fl St. Fra n cis ci; differt ab Ibide cajennensi cor̀pore minore magisque nigro, rostro pedibusque non viridibus, nucha non cristata; fronte non plumosa.

\section{Spetes 2. HBIS OXYGERCÚS. Tha LXXXVIİ}

Iİáor, nigra, violaceo renitens; periophthalmiis, gula strigaque ad latera juguli longitudinali nudis; frontë lorisque canescentibus; cauda elongata, acuità.

$\mathrm{D}$ ESCR 1 P т о: Corpus praecedentibus maius, supra stibtusque nigrum, dorso, alis caudaque violaceo-chalybeis, subvirescentibus, plumis basi nigris; caput plumosum; circa oculos naresque nudum, fronte plumosa, canescente; lora canescentia; gula strigaque juguli lateralis et longitudinalis nudae; nucha virescenti-chalybea; remiges prima- 
riae nigro-fuscae, viridi violaceoque haud renitentes, secundariae fere violaceae; alae subtus nigrae; cauda alis longis niaior, acuta, violaceo-nigra; collum subtus, pectus, abdomen femoraque fusco-nigra; pedes haud alti; rostrum tenue, cylindricum, lateraliter sulcatum. Longitudo corporis $2^{\circ}, 5^{\prime}$, caudae $1^{\prime}$, c. alis $1.3 \frac{3}{4}^{\prime}$, pedum $5 \frac{1}{2}^{\prime}$, tarsi $2 \frac{1}{2}$, , rostri $5 \frac{I^{\prime}}{2}$.

Habitat in Provincia Para.

\section{Species 3. tIBIS LEUCOPYGUS. Tab. LXXXXIIt.}

Minor, supra brunnescenti-nigra, subtus alba, plumis basi albis; collo griseo; alis subtus, uropygio, dorso inferiore caudaque basi albis.

Descriptio. Corpus praecedentibus minus, supra nigricanti-fuscum, subtus album; caput fere totum plumosum collumque brunnescenti-canescéntia vel grisea, genis, basi rostri gulaque nudis, flavicantibus; collum tenue; tectrices alarum dorsique brunneo-nigrae, haud chalybeae, basi albae; dorsum infimum, uropygium, crissum, pectus, abdomen, femora, hypochondria, alae subtus, caudaeque basis alba; cauda alis vix longior, aequalis, brunneo-fusca, basi alba ; rostrum basi sanguineum, apice supra albicans; pedes fusco-sanguinei, altiores; oculi albo-grisei. Longitudo corporis $1^{\circ} 8^{\prime}$, caudae $3 \frac{1}{2}^{\prime}$, c. alis $1.1^{\prime}$, rostri $5^{\prime}$, pedum $6^{\prime}$, tarsi $2 \frac{3}{4}{ }^{\prime}$.

\section{Familia: A r d e a e.}

Tetradactylae, plerumque macropodes, semipalmatae; rọtro capite longiore, subulato, compresso, sul.. hiante, apice acuto, marginibus oris acutis; maxilla inferiore apice ascendente; digito postico longiusculo, incumbente.

\section{Genus 1, C i c o n i a.}

In pratis paludosis, ad ripas fluminum ac lacuum amphibiis victitans, in locis altis nidificans; rostro subulato, non arcuato; naribus linearibus basalibus; collo, excepta Mycteria, plumoso; unguibus obtusis, intermedio non serrato; pedibus reticulatis. 


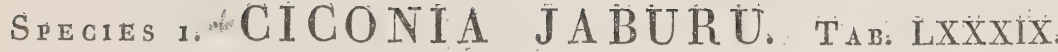

Maior, alba; regione orbitali, 'gula nec non strigis 2 versus latera jugulinudis, rubris, rostro basi virescenti, versus apicem sanguineo; remigibus tectricibusque alarum brevibus, albis, nigris intermixtis; pedibus rubris.

D S C r I t10. Corpus Ciconia europaea s. alba paulo maius, supra subtusque sordide album; caput albo-plumosum, striga infra oculos versus nares aliaque larga a mento ad latera gulae nudis, rubris; collum elongatum, parum crassum, sordide albo-plumosum, plumis versus pectus defluentibus, longiusculis; tectrices humeri axillaeque albae; dorsum, uropygium, pectus, abdomen, femora crissumque alba; remiges omnes tectricesque alarum longae, nigrae, violaceo, nec non virescenti relucentes, secundariis nonnullis albis immixtis; alae subtus antice albae; cauda alis brevior, alba, rectricibus nonnullis nigris immixtis; rostrum crassiusculum, elongatum, subulatum, compressum, basi virescens, altiusculum, medio sanguinolentum nec non hians, apice flavescens, acutum, dentatum, supra carinatum, maxilla inferiore apice ascendente, naribus basalibus linearibus; oculi flavicanti-albi; pedes alti, rubri; digiti subpalmati, haud longi, postico haud longo, subelevato, unguibus obtusis, brevibus, nigris. Longitudo corporis $3^{\circ}$, $11^{\prime}$, caudae $6 \frac{1}{3}^{\prime}$, pedum $x^{\circ}, 7 \frac{1}{2}^{\prime}$, tarsi $10^{\prime}$, rostri $8 \frac{1}{2}^{\prime}$.

Habitat in locis vel pratis paludosis Insulae St. Joannis, Rio de Janeiro et totius Brasiliae, Ja bur ú ab incolis dicta; mas a foemina non diversus; differt a Ciconia. europaea genis nuchaque magis nudis, rostro maiore, pennis caudae rigris immixtis:

\section{Geñus 2. A R D E A.}

Unguibris falcatis, ungue digiti medii introrsum serrato; rostro trigono, stibuilato, basi pauto cras= siore, usque ad oculos nudo, apice acuto, dentato; naribus subbasalibus, linearibus; sulcatis; tarsis altis, fasciato-scutellatis.

\section{Spetes 16 tARDEA MIAGUARI. Tab: XC:}

Ardeae cinereae simillima sed maior, supra plumbescens, subtus nigra; róstro flavo, capite duplo loñ giore; fronte totoque capite cristato nigris; collo albo, antrorsum medio nigro-strigilato, plumis supra pectus filosis, defluentibus; pennis dorsalibus filatis, longis, dependentibus:

D ESCRIPTIO. Corpus Ardea cinerea maits, supra plumbeum, subtus nigrum; caput compressum, cum fronte nigrum, circa oculos genasque nudum, virescens, crista occipitis 
retropendente, longa, nigra; collum elongatum, album, antrorsum medio nigro-strigilatum, pennis filosis, longis, supra pectus pendulis; dorsum plumbeum, pennis dorsi tectricumque intermediarum superjacentibus elongatis, filatis, defluentibus; humeri margoque axillaris albi ; tectrices superaxillares álbicantes, reliquae alarum plumbescentes, scapis nigris; remiges tectricesque alares nigrae vel plumbeo - nigrae, secundariae plumbeae; alae subtus cinereae; latera pectoris, hypochondria abdomenque nigerrima; pectus intermedium femoraque alba; crissum tectricesque caudae inferiores alba; cauda aequalis, alis haud maior, plumbea; rostrum flavum, capite duplo longius, compressum, ante nares sulcatum; pedes alti, virescentes; oculi flavi. Longitudo corporis $3^{\circ}, 8^{\prime}$, caudae $7^{\prime}$, rostri $7^{\prime}$, pedum $1^{\circ}, 4^{\prime}$, tarsi $7^{\prime}$.

Habitat solitaria ad ripas fl. Amazonum, mas a foemina vix diversus; differt ab Ardea cinerea fronte verticeque non albis, rostro femoribusque magis nudis, longioribus, pennisque dorsalibus elongatis defluentibus.

\section{Familia: $\mathrm{R}$ a $11 \mathrm{i}$.}

Ardeis minores, brevi-volantes, vespere frequenter vociferantes, cursores, riparii, tetradactyli, fissipedes; pedibus colloque haud elóngatis; unguihus acutis, intermedio non serrato; rostro capite paulo longiore, crassiusculo, compresso, apice declivi, non plano, subdentato, maxilla inferiore compressa, versus apicem altiore, ascendente, acuta; naribus linearibus, subbasalibus, membrana tectis; alis caudaque brevibus; digito postico longiusculo, subincumbente; tarsis digito medio vix longioribus, scutellato-fasciatis.

\section{Gevus 1. R A L L U $S$.}

Fissipes; pedibus altis; fronte nec pland nec nuda; corpore plerumque compresso; rostro apice subcylindrico, obtusiusculo, compresso, haud alto.

\section{Speqies 1 - + RALLUS ARDEOIDES. Tab. XCi.}

Maximus, nigro-fuscus, soli expostus cupreo relucens; plumis brunneo terminatis; rostro elongato, medio hiante; gula fronteque fulvescentibus; collo supra abdomineque medio nigris, albo striatis; pedibus altis.

D. E S C i p t г o. Corpus Ardea stellari maius, supra nigro-fuscum, cupreo relucens, plumis basi albis, subtus nigricans; caput nigro-fuscum, fulvo strigilatum, fronte fulvescente; gula regioque supra aures et infra oculos albescentes, fusco striatae; collum subelongatum, supra nigrum, albo striatum, subtus brunneofuscum, albo vix lineatum; 
pectus cupreofuscum; abdomen nigrum, medio albo-striatum; crissum nigrum; femora magna, nigro-fusca, introrsum albostriata, infra plus quam dimidia nuda; tectrices dorsi alarumque brunnescenti-nigricantes, cupreo-chalybeae, plumis apice fulvo terminatis; remiges nigricantes, tombaceo relucentes; alae subtus nigrae, antice albostriatae; uropygium nigrum; cauda alis paulo longior, aequalis, nigra, tombaceo splendens; rostrum nigrum, capite duplo maius, versus apicem cylindricum, subarcuatum, obtusiusculum, edentatum, subtus canaliculatum, maxilla inferiore compressa, flavicante, versus apicem nigricante, altiore, subascendente, apice declivi, naribus oblongis, membranosis, antice parum sulcatis; pedes alti, plumbeo-nigri, fasciatoscutellati, tarsis altis, digito medio paulo maioribus, digito postico longiusculo, incumbente, ungue medio non sèrrato. Longitudo corporis $2^{\circ}, 1^{\prime}$, caudae $6 \frac{1}{4}^{\prime}$, c. alis 1 . $1 \frac{8}{4}{ }^{\prime}$, pedum $9 \frac{1}{2}^{\prime}, \operatorname{tarsi} 4 \frac{1}{2}^{\prime}, \operatorname{rostri} 4 \frac{\pi}{3}^{\prime}$.

Habitat solitarius in lacubus prope pagum Contendas, vel Riachaô in Provincia Minas Geraës, aquam intrans, generi Anastomatis novo Temminkii vel Ardeae scolopaceae ac giganteae conveniens, a Rallo vero haud disjungendus.

\section{Gexus. 2. G A L L I N U L A.}

Patustris, nec non maritima, stationaria, vespere gatlinarum instar vociferans; corpore subcompresso; rostro altiore quam latiore, capite longiore, compresso, fere recto, apice subdeclivi, acuto, subdentato, basi-crassiore, planiusculo; naribus fere intermediis; unguibus haud elongatis.

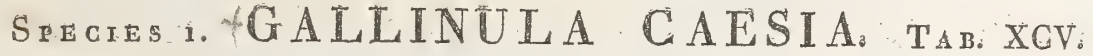

Minor, immaculata, supra olivascens, subtus et in capite plumbescens; corpore compresso; gula vix al bescente; rostro apice fere tenui.

$\mathbb{D}$ E.SCR1Pт⿺. Corpus Rallo aquatico vix minus, supra olivaceo-virescens, imma culatum, subtus totum plumbeum; caput plumbescens, vix olivascens, striga super et ante oculos albicanti; gula cinerascens; colli latera, jugulum pectusque plumbea; $a b=$ domen femoraque plumbeo vel nigricanti fusca; cauda brevis, alas vix superans crissumque nigra; tectrices alarum olivaceae; remiges fuscae; alae subtus nigrae; rostrum sulphureo-viride, fere ralloideum, basi vero altum, apice tenue, vix declive, naribus in- 
termediis, sulcatis; pedes vix alti, rubicundi. Longitudo corporis $9 \frac{\pi^{\prime}}{2}$, caudae $2 \frac{T^{\prime}}{3}$, rostri $1 \frac{3}{4}^{\prime}$, pedum $3 \frac{1}{2}^{\prime}$, tarsi $1 \frac{1}{2}^{\prime}$.

Habitat sub nomine "Saracura pequena" in Provincia Minas Gera ës prope Contendas.

\section{SPECIES 2. GALLINULA RUFICEPS. TAB. XCVI.}

IMaior, supra olivascens, subtus ferruginea; occipite rufescente; collo plumbeo; gula alba; femoribus cinereis; abdomine caudaque nigris; axillis remigibusque castaneis.

$\mathrm{D}$

E criptro. Corpus praecedente duplo maius, supra olivascens, subtus rufo-ferrugineum; caput in fronte cinerascens, in occipite rufescens; gula sordide albicans; collum supra subtusque plumbeum; dorsum tectricesque alarum viridi-olivacea; uropygium, cauda alis brevior, abdomen posterius crissumque nigerrima; pectus, abdomen superius hypochondriaque rufo-ferruginea; femora cinereo-nigricantia; tectrices humerales, scapulares longaeque olivaceae, superaxillares rufescentes, axillares remigesque cauda longiores castaneae, apice vix olivascentes; alae subtus rufae, antice nigro rufoque-albo fasciatae; rostrum compressum, basi flavicans, apice virescens; oculi flavocastanei; pedes rubicundi. Longitudo corporis $I^{\circ} \frac{1}{3}^{\prime}$, caudae $3^{\prime}$, rostri $2^{\prime}$, pedum $5^{\prime}$, $\operatorname{tarsi} 1 \frac{1}{2}$.

Habitat in Provincia Rio de Jan eiro, Gallinulae cayannensi Lath. et Buff. similis, occipite vero rufescenti diversa.

\section{Species 3. GALLINULA IMANGLE. Tas. XCViI.}

Praecedente minor, supra olivaceo-, subtus ferrugineo- rufa; collo subtus rufo, supra olivaceo-plumbeo; capite olivaceo; maxilla inferiore apice vix alta.

$\mathrm{D}$

escri tr o. Corpus Gallinula caesia maius, reliquis vero minus, olivascens supra, subtus rufum; caput fusco olivascens, in fronte cinerascens; gula loraque albicantia; collum subtus rufum, supra plumbescens, olivaceo indutum; dorsum tectricesque alarum pallide olivacea; axillae olivascentes; tectrices superaxillares, rufescentes; remiges castaneae, apice olivascentes, secundariae superjacentes olivascentes; alae subtus rufae, antice nigro alboque fasciatae; pectus, hypochondria abdomenque superius 
rufa; uropygium, crissum, abdomen inferius caudaque alis brevior nigerrima; femora fusco-cinerascentia; rostrum haud altum, sulphureo flavicans, supra apice fuscescens, maxilla inferiore apice vix altiore; pedes rubicundi, digitis fuscis. Longitudo corporis $11^{\prime}$, caudae $2 \frac{1}{4}^{\prime}$, rostri $1 \frac{3}{4}^{\prime}$, pedum $5^{\prime}$, tarsi $2^{\prime}$.

Habitat ad litora maris in locis paludosis, arbustis mangliferis obsilis。

\section{Species. 4" tGALLINULA SARACURA. Tan. XCVIII،}

Maior, supra olivacea, subtus tota plumbea; collo supra castaneo; occipite fusco-rufescente; gula parum alba; rostro viridi; remigibus fusco-rufis.

D

Escriptro. Corpus praecedentibus maius, subtus totum plumbeum; caput crassiusculum, antice plumbescens, postice fusco-brunnescens, gula alba; lora cinerea; collum supra laete castaneum; dorsum tectricesque alarum olivaceo-viridia; axillae brunnescentes; remiges fusco-castaneae, vexillo interno fusciore, secundariae superja= centes olivascentes; alae subtus fusco-rufae, antice nigro rufoque fasciatae; pectus, ab= domen superius femoraque plumbea; abdomen inferius, crissum caudaque alas vix su= perans nigerrima; rostrum longum, viride, compressum, supra basi planiusculum; pedes fusco-rubicundi. Longitudo corporis $x^{\circ} \frac{x^{\prime}}{3}$, caudae $2 \frac{{ }^{\prime}}{2}$, rostri $2 \frac{1^{\prime}}{}{ }^{\prime}$, pedum $5 \frac{1^{\prime}}{4}$, tarsi $2 \frac{3}{4} \cdot$.

\section{Spectes 5. +GALLINULA GIGAS. Ta Ẋ XCIX。}

Maxima, olivascens; capite, collo subtus pectoreque plumbeis; collo supra castaneo; abdomine superiore et hypochondriis pallide rufescentibus; cauda alis longiore.

$\mathrm{D}$ E S C R I P т о. Corpus, respectu praecedentium, maximum, variegatum; caputcrassum, cinereum, postice brunnescens, striga ante et super oculos canescente; lora collumque subtus pectusque plumbea; gula albicans; collum posticum castaneum; dorsum rectricesque alarum olivaceo-virescentes; dorsum inferius, uropygium, cauda alis longior, abdomen inferius crissumque nigerrima; abdomen anterius et hypochondria brunnescentiferruginea; femora cinerascentia; axillae castaneae, vix olivascentes ; remiges castaneae, secundariae brunneo-olivascentes; alae subtus rufae, antice nigro fasciolatae; rostrum longum, compressum, sulphureo virescens, basi supra planiusculum, maxilla inferiore 
versus apicem angulato-elevata; pedes rubicundi. Longitudo corporis $x^{\circ}, 6^{\prime}$, caudae $3 \frac{3}{4}^{\prime}$, c. alis 1. $1 \frac{1}{2}^{\prime}$, rostri $2 \frac{3^{\prime}}{4}$, pedum $6^{\prime}$, tarsi $3^{\prime}$.

Habitat, Saracura Grande nominata, in locis paludosis Provinciae interioris M inas Geraës, prope pagum Contendas, vespere perambulando vociferans.

\section{Familia: T r i n g a e.}

Tetradactylae, scolopacibus affines, ripariae, cursores, circumvagae, agiles, voce vix tinnientes; digito postico brevi, non incumbente; rostro recto, tenui, haud alto, subcylindrico, paulo longiore, apice planiusculo, mandibula inferiore apice non ascendente; naribus basalibus, linearibus, sulcatis; alis longiusculis, cruciatis; axillis non aculeatis sed tuberculatis; tarsis digito medio longioribus, scutellato-fasciatis.

\section{Speqes i. TRINGA MAGROPTERA. Tas. Xcit.}

Minor, supra fusco-brunnea, albo punctata, subtus alba; jugulo obfuscato; remigibus nigris; axillis subtus caudaque alis breviore albo nigroque fasciatis; striga ante oculos alba; rostro capite longiore, apice cylindrico.

$\mathrm{D}$ Escripro. Corpus Tringa Cinclo paulo maius, supra et in jugulo fusco-brunneum, subtus album; caput jugulumque fusco brunnescentia, striga ab oculis ad nares alba; gula, pectus, abdomen, hypochondria, crissum femoraque alba; dorsum pallide brunnescens, albo punctatum; tectrices axillares nigrae, reliquae alarum pallide brunnescentes, plumis vix fulvo terminatis; remiges primariae nigrae, longae, cruciatae, secundariae pallide brunnescentes; alae subtus antice albo nigroque variegatae; cauda alis brevior, subaequalis, alba, nigro 4-vittata, apice albo terminata; rostrum nigrum, capite paulo longius, tenerrimum, ante nares sulcatum, apice cylindricum, declive et vix planum; oculi nigri; pedes virescentes, digito externo basi semipalmato, interno libero. Longitudo corporis $7 \frac{3{ }^{\prime}}{4}$, caudae $1 \frac{3{ }^{\prime}}{4}$, rostri $1 \frac{{ }^{\prime}}{4}$, pedum $2^{\prime}$, tarsi $13^{\prime \prime}$.

Habitat ad litora maris in Provinciis Rio de Janeiro et Bahia.

\section{Speres 2. TRINGA BREVIROSTRIS. тав. XCI.}

Minima, supra pallide fulvo-brunnescens, non maculata, subtus et in fronte tota alba; alis subtus scapisque remigum aibis; rostro nigro, capite fere breviore, apice plano.

Descri т то. Corpus Tringa pusilla haud maius, supra pallide brunnescens, non maculatum; subtus totum album, plumis basi nigris; caput supra pallide brunnescens, 
vix fusco maculatum, fronte, genis lorisque albis; tectrices axillares et humerales nigricantes, reliquae alarum brunneo-canescentes, albo fimbriatae; remiges primariae nigro-fuscae, basi albae, scapis albis; alae subtus albae; cauda alis vix longior, fulvoalbo canescens, rectricibus 2 intermediis nigris albisque immixtis; uropygium nigricans; rostrum nigrum, breve sed maius quam in Tringa pusilla, apice planiusculum, triquetrum; pedes nigri, semipalmati; digiti breves, fimbriati, posticus brevis, tenerrimus. Longitudo corporis $5^{\prime}$, caudae $1 \frac{3^{\prime}}{4}$, c. alis $1 . \frac{1}{2}^{\prime}$, pedum $2^{\prime}$, tarsi $9^{\prime \prime}$, rostri $9^{\prime \prime}$.

Habitat in Brasilia, Tringae oceanicae praecipue albescenti Temm. pl. $4 \mathrm{r}$ similis, ob descriptionem vero brevem haud distinguenda.

\section{Familia: C H A R A D R I I.}

Tridactyli, riparii, cursores, circumvagi; capite crassiusculo; digitis brevibus, externo semipalmato, unguibus teneris, acutis; tarsis digito medio duplo longioribus, reticulatis; rostro haud crasso, supra basi non plano, medio cavo, versus apicem plerumque tumido, convexo, apice subdeclivi, acuto, maxilla inferiore fere recta, compressa, subaequali; naribus intermediis, membranosis.

\section{Genus 1. C H A R A D R I U S.}

Rostro capite haud longiore, fere recto, versus apicem intumescente; maxilla inferiore apice subascendente; alis cruciatis, longiusculis.

\section{Species i. CHARADRIUS CRASSIROSTRIS. Tab. XCIV.}

Minor, supra brunneo canescens, subtus albus; collari capiteque fuscis; fronte, superciliis colloque medio albis; rostro versus apicem crasso.

D Esc in т ro. Corpus Tringa pusilla vix maius, supra fusco-canescens, subtùs album; caput pallide brunnescens, fronte, genis superciliisque albis; gula collarique albis; lora collareque supra pectus fusco-brunnea; dorsum tectricesque alarum albicantibrunnescentes; remiges nigro-fuscae, basi albae, scapis albis; axillae spinoso-tuberculatae; alae subtus totae albae; cauda alis haud maior, rectricibus internis nigro-brunneis, externis albis; rostrum fuscum, capite fere brevius, versus apicem crassum, cylindricum, subdentatum; pedes rubri, digitis brevibus. Longitudo corporis $7 \frac{1}{3}$, caudae $2^{\prime}$, rostri $10^{\prime \prime}$, tarsi $13^{\prime \prime}$, pedum $2^{\prime}$.

Habitat sub nomine vago "Ma sa $\mathrm{r}$ in ho" ad ripas fluviorum. 


\section{Familia: F U L I C A E.}

Tetradactylae, vix volantes; digitis lobatis; tarsis compressis; rostro subulato, subcompresso; alis brevibus; cauda fere nulla.

\section{Genus 1. P O D I C E P S.}

Lobis digitorum haud emarginatis, basi connatis; tibia compressa, postice serrata; alis caudaque brevissimis; unguibus planis, rotundis; rostro recto, compresso, versus frontem haud nudo.

\section{Spetes . PODICEPS CAROLINENSIS. Tав. C.}

Mediocris, supra brunneo-nigricans, subtus albescens; gula large nigra, lateraliter albo marginata; rostro medio nigro - fasciato; jugulo intermedio brunnescente, infimo nigricante; remigibus pal. lide brunneis, basi subtusque albis.

Descripio. Corpus magnitudine fere Gallinulae Chloropi, supra brunneo-nigricans, subtus albo-sericeum, nigro maculatum; caput supra nigro-fuscum, supra aures, praecipue ad latera menti canescens; gula large nigro-maculata; collum longiusculum, postice nigricans vel brunneum, antice medio fulvo-brunnescens, infra albo nigroque variegatum; pectus abdomenque intermedia albo-sericea, lateraliter nigro-fusca; femora nigro-fusca, antice supra vix rufescentia; dorsum fusco nigricans; tectrices alarum brunneo-fuscae; remiges haud longae, albo-brunnescentes, introrsum, basi subtusque albae; cauda quasi nulla; rostrum albicans, compressum, apice subdeclive; lingua apice paulisper laciniata; tarsi nigri, digito medio breviores, compressi, postice serrati; digiti nigri, argenteo induti, usque ad medium lobati, basi uniti, externus fere reliquis longior, posticus sublobatus, unguibus plano-rotundis. Longitudo corporis $11^{\prime}$, pedum $2^{\prime}$, tarsi $1^{\prime}$, rostri $1 \frac{1}{3}^{\prime}$.

Habitat in locis paludosis Provinciae Rio de Janeiro.

\section{Species 2. PODICEPS DOMINICUS. Tab. CI.}

Minor, supra nigricans, subtus albo-sericeus, vix rufo indutus; remigibus fere omnibus albis; mento albo; capite, genis, loris, gula colloque supra nigricantibus.

D ESCRIPro. Corpus Podicipite minore fere minus, supra non brunneum sed nigricans, subtus albo-sericeum, nigro maculatum rufescentique nebulatum; caput ni- 
gricans, vix cristatum, supra aures cinereo-fuscum; mentum albicans; gula nigricans vel nigro variegatum; collum posticum nigricans, anticum brunnescenti fuscum; dorsum tectricesque alarum fusco-nigra, immaculata; remiges, praecipue secundariae albae, margine primariarum exteriorum externo apiceque fuscis; alae subtus albae; pectus abdomenque sordide albo-sericea, cinereo nonnihil variegata, vix rufescentia, macula interdum abdominis medii nigra; femora fusco-nigra; cauda fere nulla; hypochondria albicantia; rostrum breve, brunneo nigroque varium, apice albicans; tarsi nigri, postice longitudinaliter biserrati; digiti usque ad medium lobati, externus lateràlis intermedio fere aequalis. Longitudo corporis $6^{\prime}$, pedum $1^{3} \frac{3}{4}^{\prime}, \operatorname{tarsi} 1^{\prime}$, $\operatorname{rostri} 11^{\prime \prime}$.

Habitat solitarius et vix volans in aquis fluviatilibus et stagnantibus Provinciae St. $\mathrm{Pauli}$ et Minas Gera ës, uti plurimae aves aquaticae specrbus reliquarum mundi partium communis; differt a Podicipite minore gula non tota alba, dorso non brunneo, remigibus primariis non nigro-fuscis, a Podicipite insulae Trinitatis gula non tota nigra et hypochondriis non nigris. 


\section{Ares aquaticae.}

Tetra-, raro tridactylae, palmatae, excepto Tachypede supra genua nudae, in aqua piscibus victitantes, frequenter familiares, migratoriae, haud vivaces ac dociles; pedibus brevibus; corpore dense plumoso; digitis palmatis; rostro frequenter recto, apice variante.

\section{Familia: L a r i.}

Tetradactyli, palmati, supra aquae superficiem volitantes vel natantes vel in petriset ripis arenoșis nidificantes ac gregatim sedentes; corpore non recto sed obliquo; digitis gracilibus, 3 anticis totis, vel fere totis palmatis, medio paulo longiore, postico brevi, fere lateraliter posito, libero; alis elongatis, falcatis, cruciatis; rostro subcompresso, frequenter acuto, declivi, maxilla inferiore apice ascendente.

\section{GENUS 1. RHYNCHOPS.}

Rostro longo, quam maxime compresso, tenui, acute cultrato; pedibus digitisque brevibus, paulo plus quam semipalmatis; digito postico laterali, libero; naribus basalibus, fere inferis.

\section{Stétes. 1. RHYNCHOPS CINERASCENS. Tав. CII.}

Supra nigro-fuscus, subtus albus; collo caudaque supra cinerascentibus; tectricibus alarum fulvo vel albo fimbriatis.

D

ES CRIPTio. Corpus Rhynchope nigro vix minus, supra cinereo-vel nigricanti fuscum, subtus albissimum; caput supra cinereo-fuscum, fronte rostrique basi tota albis; tectrices scapulares nigricantes, reliquae alarum cinereo-fuscae, pallide fulvo terminatae, longae, apice albae; remiges elongatae, falcatae, nigrae, vexillo interno canescente; alae subtus albae; gula, pectus, abdomen, femora crissumque alba; cauda apice furcata, alis multo brevior, supra pallide albescens vel subbrunnescens, subtus basique alba, apice albo-fimbriata; uropygium nigro-fuscum; rostrum capite duplo longius, nigrum, basi rubicundum, naribus pone membranosis; pedes rubicundi. Longitudo corporis $1^{\circ} 7^{\prime}$, caudae $4 \frac{1}{2}^{\prime}$, rostri $4^{\prime}$, pedum $2 \frac{1}{2}^{\prime}$, tarsi $12^{\prime \prime}$.

Habitat in locis ripariis fl. A mazonum; differt a Rhynchope nigro corporis supra et tectricum alarum colore; an varietas aetatis? 


\section{SPECIES 2. RHYNCHOPS BREVIROSTRIS. Tав. CIII.}

Minor, subtus albus, supra nigricans, plumis omnibus fulvo terminatis; capite toto colloque supra fulvis; rostro capite vix longiore, aequali, basi haud tuberoso; alis cauda vix longioribus.

$\mathrm{D}$

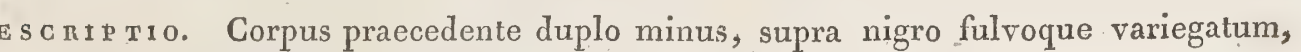
plumis nigris, apice large fulvo-fimbriatis, subtus albissimum; caput totum fulvum, nigro maculatum, fronte fulva; tectrices dorsi alarumque fulvo terminatae, alarum longae apice albescentes; remiges nigrae, secundariae rufo-albo fimbriatae; uropygium albescens; cauda alis vix brevior, subfurcata, supra brunneo-fusca, rectricibus fulvofimbriatis, subtus cinerascens; rostrum capite vix longius, aequale, nigrum, basi haud. crassius, rubescens. Longitudo corporis $10 \frac{I^{\prime}}{2}$, caudae $2^{\prime}$, rostri $1^{\prime}, 11^{\prime \prime}$.

An juvenis speciei praecedentis, rostro pedibusque licet osseis?

\section{GENU.2. S T E R N A.}

Rostro subcylindrico, apice acuto, compresso, haud adunco; naribus haud tubutosis sed late= ralibus et linearibus; digito postico minuto, sublaterali; alis longis, forficatis; pedibus abbreviatis.

\section{Spetes.x. + STERNA IMAGNIROSTRIS. Tab. CIV.}

IMediocris, plumbeo-cinerea supra, subtus alba; capite remigibusque nigerrimis; stria genarum tectrici= busque alarum intermediis albis; rostro flavo, crassiusculo.

D

escripto. Corpus Laro ridibundo minus, supra nigricanti-cinereum, vel plumbescens, subtus albissimum; caput supra totum nigrum, stria a fronte infra oculos alba; collum posticum medium fere album, infimum nigro-cinerascens; dorsum tectricesque scapulares plumbeo-cinerea; tectrices humerales, axillares alarumque mediae albae, supraaxillares nonnullae nigrae; remiges nigrae, elongatae, furcatae, vexillis internis basi albicantibus, secundariae albicantes, cinereis immixtis; cauda subbifurcata, alis minor, supra plumbeo-nigricans, subtus pallide plumbescens; rostrum capite duplo maius, flavum, basi cinerascens, crassiusculum, convexum, apice compressum, acuminatum, naribus fere linearibus, subbasalibus; oculi flavi; pedes nigri. Longitudo corporis $1^{\circ}, 5^{\prime}$, caudae $3 \frac{1}{3}^{\prime}$, rostri $3 \frac{1}{3}^{\prime}$, pedum $1^{\prime}, 10^{\prime \prime}$, tarsi $10^{\prime \prime}$.

Habitat in lacubus fl. St. Francisci, a cl. Lichtensteinio magnirostris nominata. 


\section{Familia: $\mathrm{P}$ e 1 e c a $\mathrm{n}$.}

Tetrąactyli, steganopodes, haud raro alte volantes; digitis 4 anticis membrana palmari oblique conjunctis; axillis elongatis; corpore obliquo; gula nuda; rostro edentulo, longo, subcylindrico, supra basi largiusculo, frequenter adunco, gonyde deflexa, rictu oris usque infra oculos prolongato; naribus basalibus, linearibus, minutis, antice sulcatis; ungue digiti medii, excepto Onocratalo, pectinato; tibia digitis breviore, reticulata.

\section{Genus 1. T A C H I P T E S.}

Rostro longo, superiore et inferiore aduncis; alis caudaque elongatis, furcatis, remige rectriceque exterioribus longissimis; tarsis brevissimis, rotundis, semiplumosis; digitis 4 unitis, nonnisi semipalmatis, intermedio longiore; collo subtus fere toto nudo.

\section{Speies 1. + TACHIPETES AQUILA. Taв. $\mathrm{TV}$.}

Maximus, niger, cupreo-, nec non virescenti renitens; cauda nigra, longissima, furcata; tibiis totis plumosis.

$\mathrm{D}$ crassum, nigrum, plumis occipitis acuminatis, virescentibus; collum subtus medium a mento usque fere ad pectus nudum, rubicundum; dorsum violaceo-cupreo splendens; alae supra nigrae, virescenti-violaceo subrelucentes, subtus atrae; axillae prolongatac; rèmiges longissimae, cruciatae, nigrae, secundariae superiores apice brunnescentes, remige primaria externa longissima; cauda alis longior, furcata, nigerrima, basi subalbicans, vexillis internis basi clare brunnescentibus rectriceque externa longissima, scapis subtus albis; rostrum capite triplo fere longius, nigrum, apice compressum, supra et subtus aduncum, lateraliter usque ad nares lineares sulcatum, supra versus frontem planiusculum; pedes brevissimi, retropositi, fusco-rubri; tarsi toti et large plumosi; digiti haud crassi, omnes 4 membrana palmari usque ad dimidium uniti, posticus lateralis cum reliquis unitus, medius reliquis longior ungue introrsum pectinato. Longitudo corporis $3^{\circ} 3^{\prime}$, caudae $1^{\circ} 5^{\prime}$, c. alis $1.3 \frac{1}{2}^{\prime}$, alarum $2^{\circ}$, pedum $1 \frac{3}{4}^{\prime}$, rostri $5^{\prime}$.

Habitat ad litus maritimum $\mathrm{R}$ io $\mathrm{de} J$ a $\mathrm{n}$ eiro nec non $\mathrm{B}$ ahiae, in aere regionum a mare interdum remotarum pendens; foemina vel juvenis rostro albo, capite, collo, pectore, abdomine, nec non scapulis albicantibus, nigro maculatis. 


\section{Genus 2: $\mathrm{G}$ A $\mathrm{R}$ B $\mathbf{0}$.}

Rostro adunco, versus oculos angustato; digitis 4 anticis totis palmatis, obliquis, externo longiore; gula modo tarsisque nudis; alis breviusculis non furcatis; scapis caudae rigidioribus; tarsis compressis.

\section{Sizeres. 1. + CARBO BRASILIANUS. Ta b. GVI.}

Niger, supra brunnescenti submaculatus, Carboni Graculae similis sed minor; gula vix nuda, albo non cincta; cauda longiuscula.

Descript o. Corpus Pelicano Carbone paulo minus, nigrum, plumis acuminatis supra dorsum et alas medio brunnescentibus nigroque marginatis; caput elongatum, vix crassum, nigrum, ante et infra oculos nudiusculum; collum maris nigrum, foeminae subtus usque post pectus brunnescens; gula vix nuda; plumae dorsi infimi nigerrimae, nec maculatae nec acuminatae; remiges haud longae, fusco-nigrae, secunda externa longiore, secundariae breves; alae subtus nigrae; pectus, abdomen, femora usque ad genua plumosa crissumque nigra; cauda nigra, acuminata, minus rigida, alis multo longior; rostrum supra nigricans, subtus flavicans, infra oculos compressum; rictu oris usque fere ad aures prolongato; pedes robusti, compressi, nigri; digiti oblique positi, exterior lateralis, reliquis longior, ungue medio pectinato. Longitudo corporis $2^{\prime}, 3^{\prime}$, caudae $7^{\prime}$, c. alis $1.4^{\prime}$, rostri $3^{\prime}$, pedum $3^{\prime}$, tarsi $2^{\prime}$.

Habitat gregarius in lacu Coari; differt a C. Gracula europaea gula haud large nuda, nec albo cincta, plumis dorsi anterioris magis longis, caudaque maiore.

\section{GENUS 3. S U L A.}

Rostro recto, non adunco, ad margines oris supra subtusque serrato, basi crasso, supra frontem plano-convexo, gonyde subascendente; digitis 4 anticis membrana oblique unitis, medio fere longiore, ungue medio pectinato; naribus nullis vel non conspicuis.

\section{Species 1. SULA BRASILIENSIS. Taв. CVII.}

Maior, clare brunnescens, immaculata; abdomine medio vix albescente; remigibus rectricibusque longiusculis, fuscis.

$\mathrm{D}$ ESGR I to. Corpus Carbone brasiliano vix minus, immaculate et clare brunnescens; caput brunnescens, ante, supra et infra oculos compressum, nudum et flavescens; 
remiges nigro-fuscae, longae, acutae, secundariae minores, brunnescentes, canis nonnullis immixtis; pectus abdomenque brunneo-canescentia, plumis basi albicantibus, apice albo punctulatis; cauda fusca, alis vix longior; rostrum virescens, basi crassum et supra plano-convexum, lateraliter sulcatum, apice compressum, acutum, non aduncum, marginibus oris denticulatis, denticulis retroversis, naribus extus non conspicuis, gonyde subascendente; pedes flavi, haud alti, subdepressi; digiti 4, toti palmali, oblique positi, medius sublongior ungue pectinato.

Habitat in insulis maritimis urbis Rio de Janeiro.

\section{Familia: A n s e r e s.}

Tetradactyli, palmati, non steganopodes, lamellirostres, frequenter in aquis dulcibus piscibus, insectis vel herbis victitantes; digitis 3 anticis palmatis, non oblique positis, medio longiore, postico libero; rostro lamelloso-dentato, depresso, lato, apice unguiculato; corpore obliquio, ponderoso; pedibus brevibus; lingua ciliata.

\section{Genus 1. A N S E R.}

Statura, tarsis, praecipue collo altioribus; dentibus haud marginalibus sed large transversis; naribus oblongis; rostro apice angustiore, subcylindrico, latere recto, basi altiore; tarsis digito medio longioribus.

\section{Species 1, -ANSER JUBATUS. Taв. CVIII.}

Mediocris, variegatus; capite, collo, pectore, crisso speculoque alarum albis; collo postico jubato; abdomine dorsoque rufis; alis caudaque violaceo-nigris; axillis spinosis.

D S C 1 P т 1 0. Corpus Anate Boschate maius, albo, nigro, rufoque varium; caput, collum pectus, crissum speculumque alarum clare vel sordide alba; occiput collumque posticum brunnescenti-alba, jubata; dorsum anterius nigrum, rufo-maculatum, plumis longiusculis; dorsum inferius uropygiumque violaceo-nigra; abdomen hypochondriaque castaneorufa; femora rufescenti-cinerea; alae nigrae, viridi splendentes, speculo albo; remiges nigricantes, secundariae brunneo-nigrae, subvirescentes, primariis adjacentes medio albae; axillae tuberculatae vel spinosae; alae subtus nigrae; cauda aequalis, violaceo-nigra; rostrum nigrum, capite vix longius; oculi brunnei; pedes rubri. Longitudo corporis $1^{\circ}, 10^{\prime}$, caudae $5^{\prime}$, c. alis $1.2 \frac{1}{3}^{\prime}$, rostri $1 \frac{3}{4}^{\prime}$, pedum $5 \frac{1}{2}^{\prime}$, tarsi $3^{\prime}$.

Habitat ad ripam fluminis Solimoëns in insula "Praya das Onças." 


\section{GeNus 2. A N A S.}

Rostro breviore, lato et magis depresso, ad latera obliquo, unguiculo apicis longiusculo; dentibus lamellosis, longis, transversis; naribus rotundioribus; collo pedibusque brevioribus; tarsis digito medio brevioribus.

\section{Species 1. ANAS PATURI. Mas. Tab. CIX.}

Minor, brunnescens; capite antico gula colloque inferiore rufis; occipite nigro; collo medio albescenti; humeris nigris; speculo alarum viridi-splendente, postice albo.

$\mathrm{D}$ ESCRIPTro. Corpus Anate Boschate duplo minus, supra subtusque umbraceo-brunnescens; caput antice, super genas et infra gulam rufum, in occipite nigrum; collum superius sordide albicans, inferius rufum, fusco vix maculatum; pectus albescens, nigro vel brunneo-fusco variegatum; abdomen crissumque brunneo-cinerascentia; dorsum anterius pallide brunnescens, posterius nigerrimum; uropygium pallide-brunnescens; alae antice supra subtusque nigerrimae, subviolaceae, mediae viridi-, pone violaceo splendentes, postice nigro alboque marginatae, tectricibus viridibus, acuminatis; remiges nigricantes, subvirescentes, secundariae pallide brunnescentes, secundaria exteriore extrorsum nigro-fimbriata; alae subtus cinereo-atrae; cauda breviuscula, acuminata, nigra, violaceo-viridi nebulata; rostrum fusco-castaneum, apicis unguiculo medio brunneo; pedes rubri. Longitudo corporis $1^{\circ}, 1^{\prime}$, caudae $3^{\prime}$, c. alis $1.1^{\prime}$ ', rostri $1 \frac{3}{4}$, tarsi $1^{\prime}$, pedum $2^{\prime}$.

Habitat in lacubus prope flumen St. Francisci. 




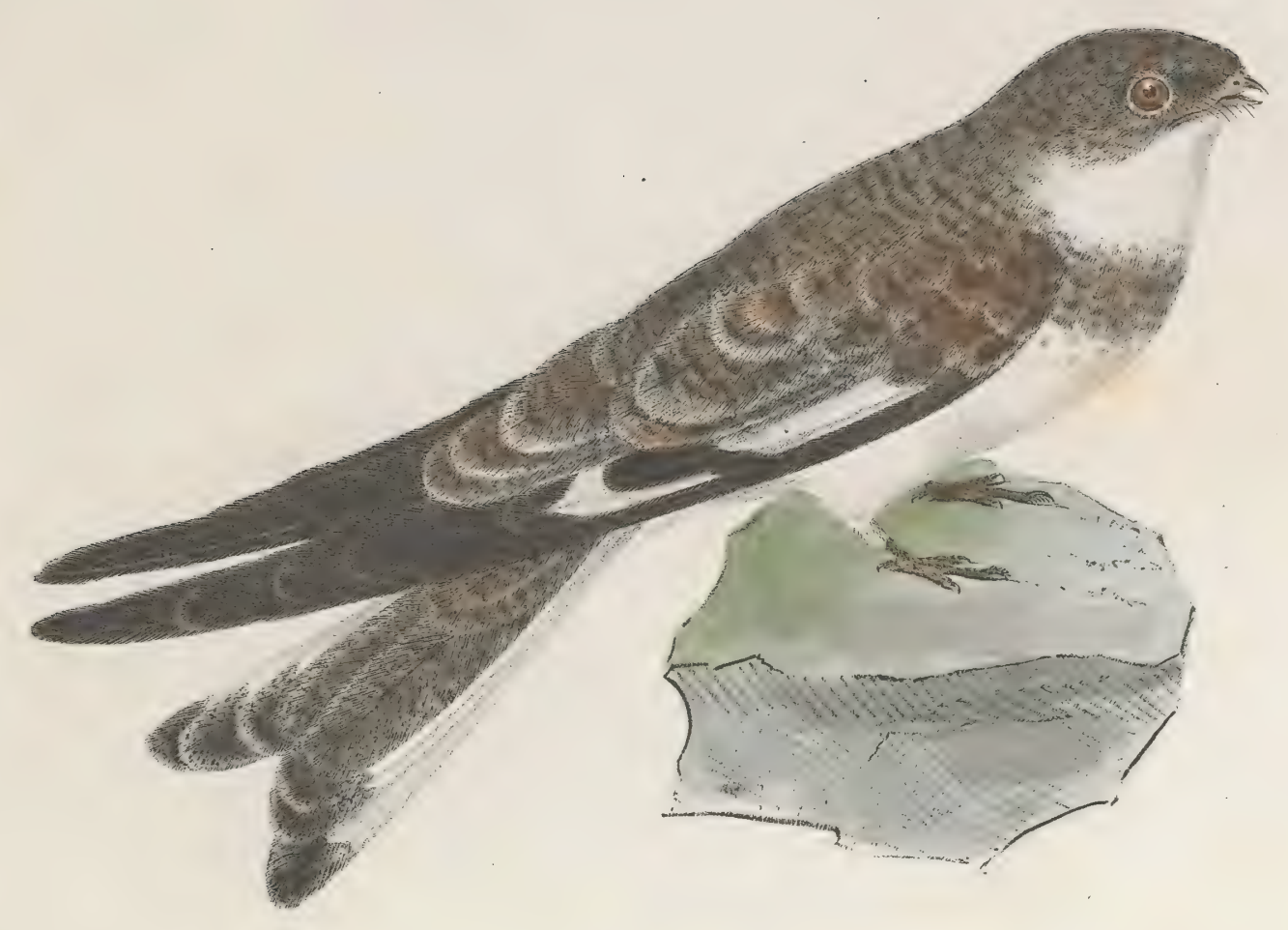

+ CAPPRRMIUILGUSS rupestris.

Tab.II . 



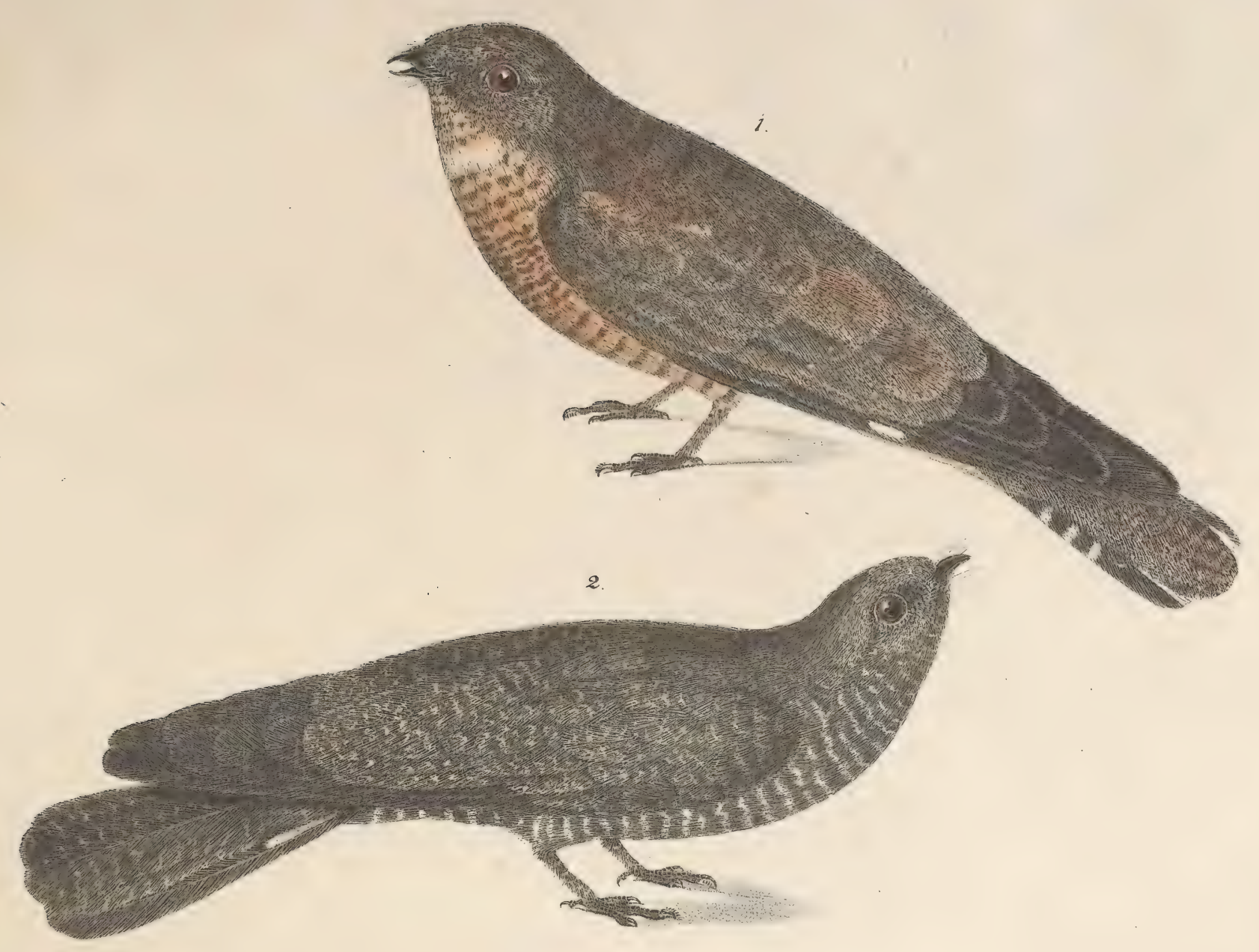

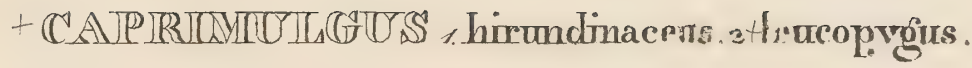

Tab.JII . 



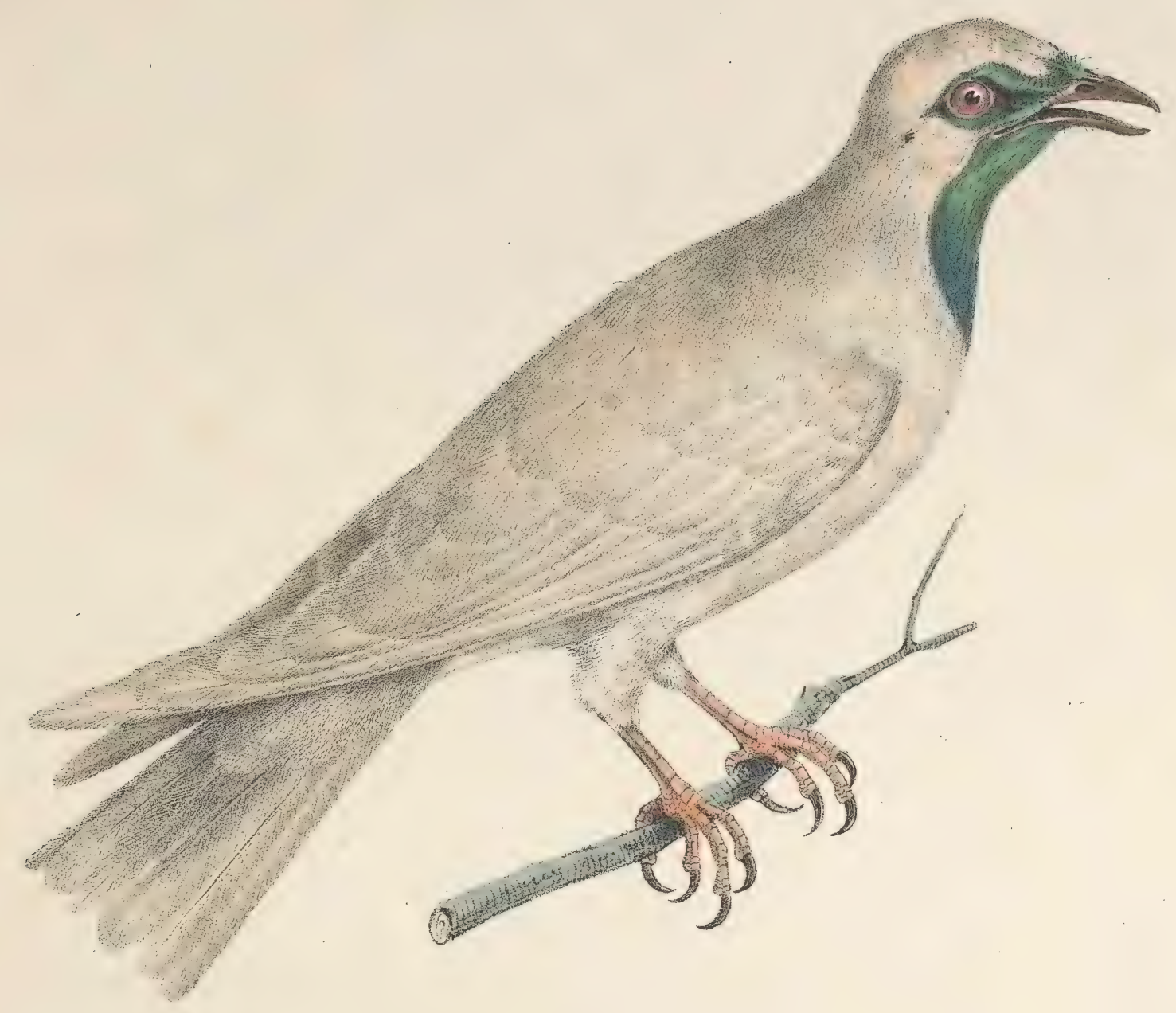

+ CASAMEARTERTCHUS e carunculatus.

Tab.IV. 



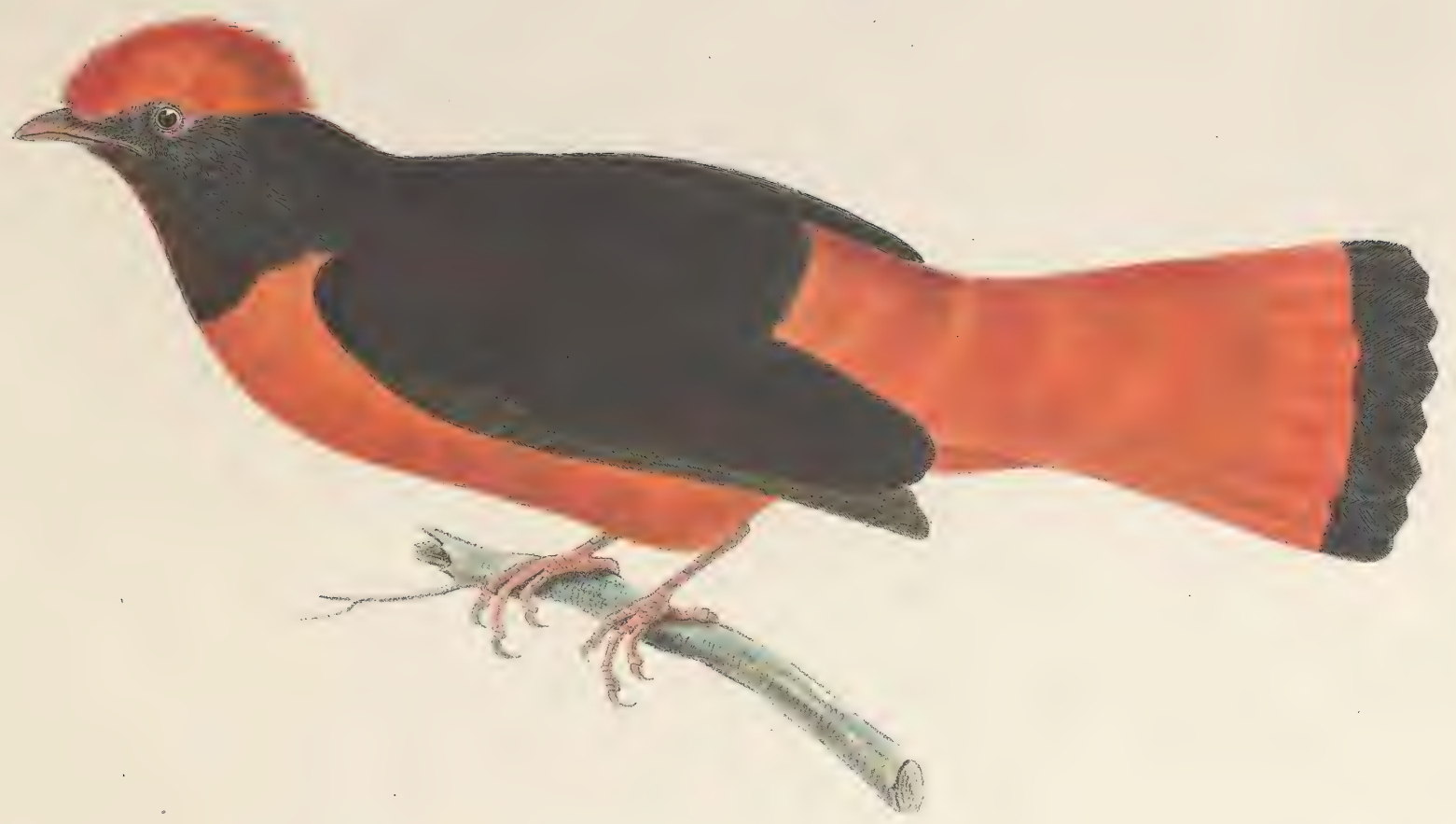

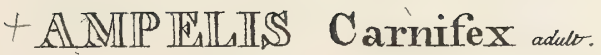

Tab.V. 



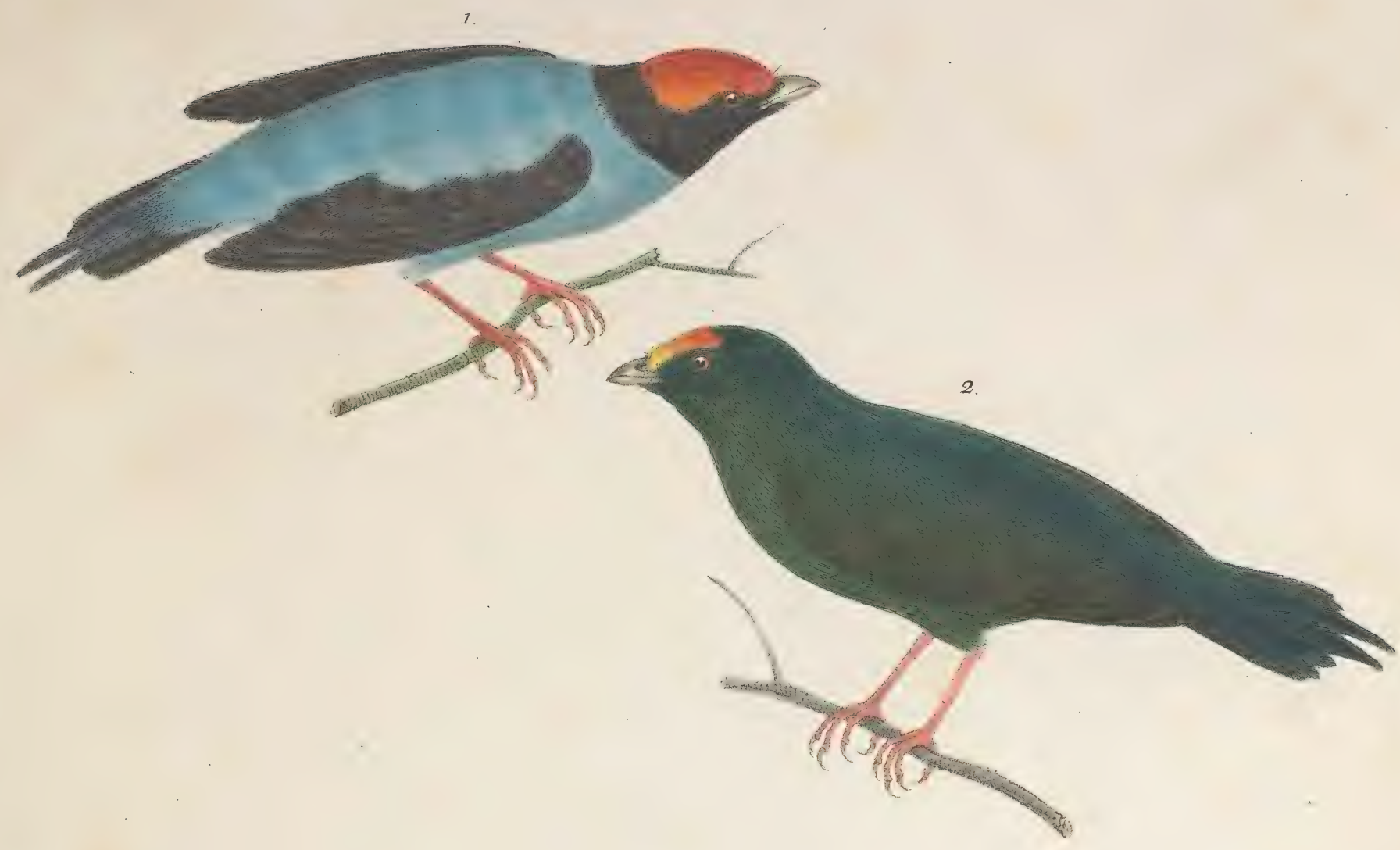

+ IPIIPIRA caudata 1. מus.2.sums

Tab.VI. 


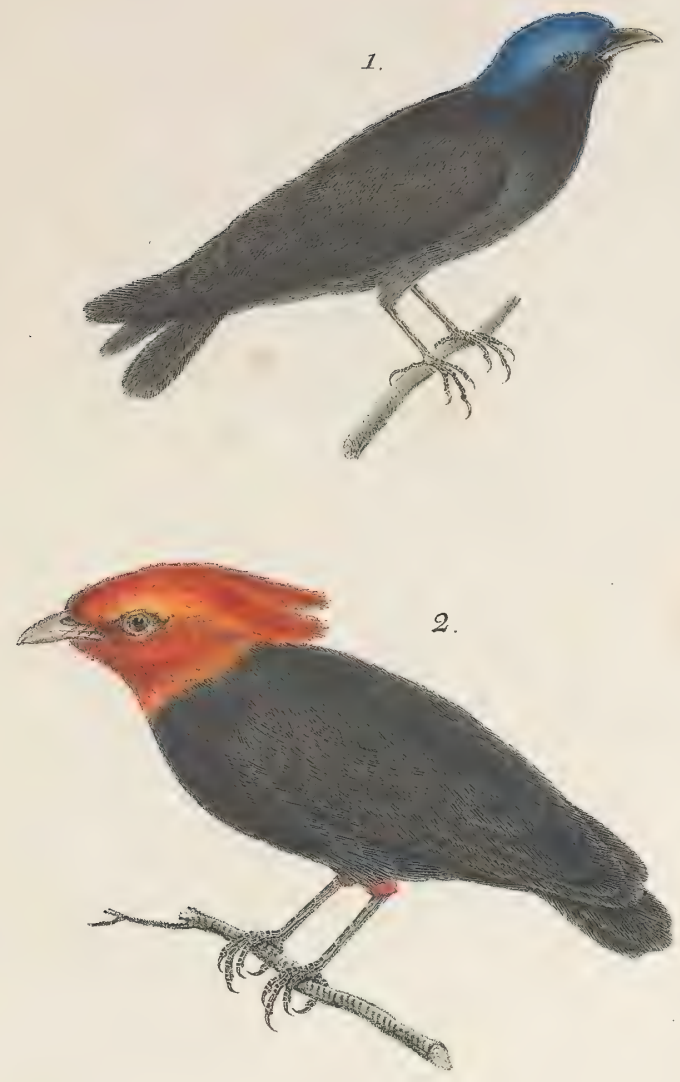

IPIIPIRA 1.coronata.z cornuta.

Tab.VII. 



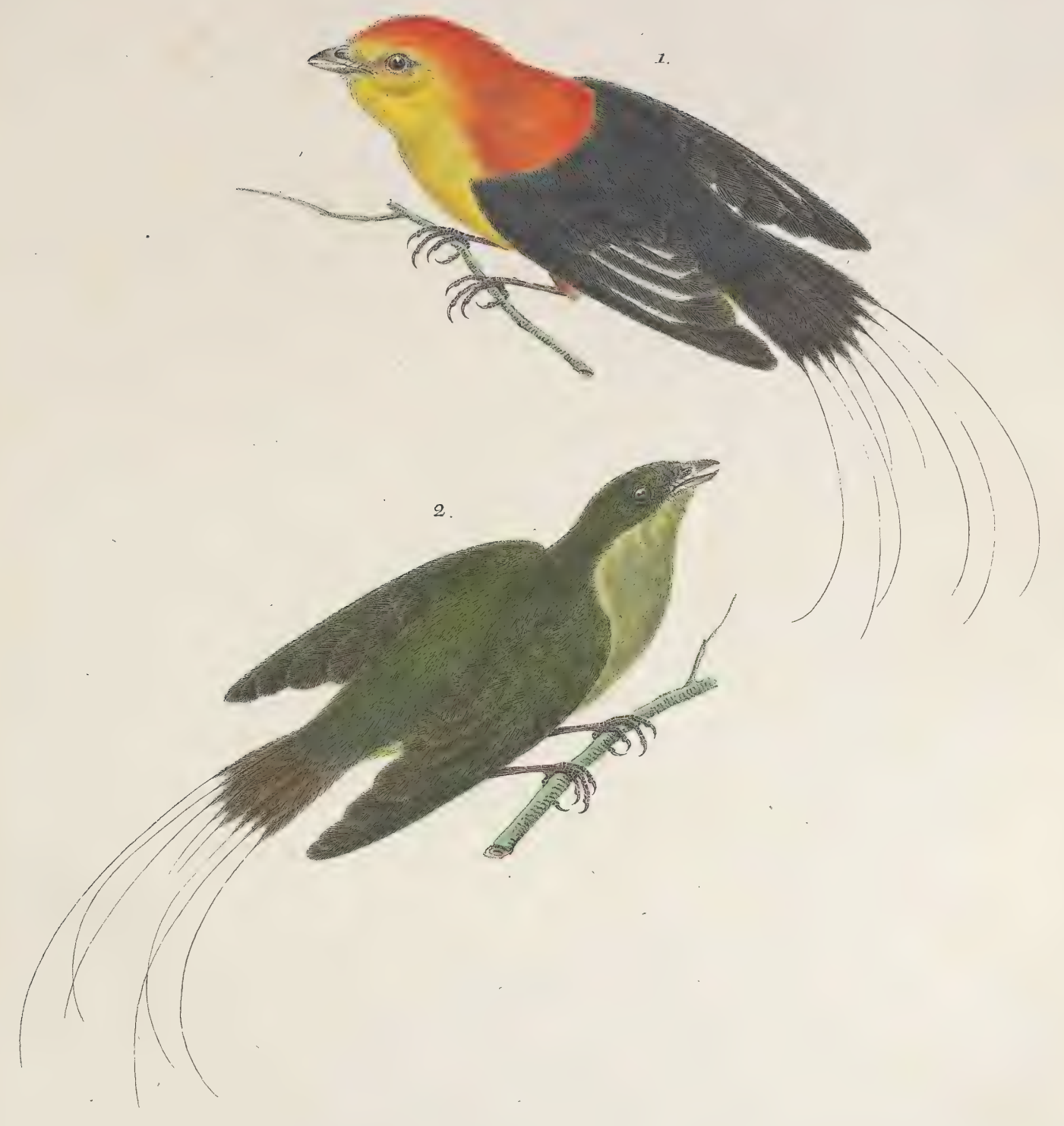

TPIIPTRA Filicauda.

Tab . VIII. 


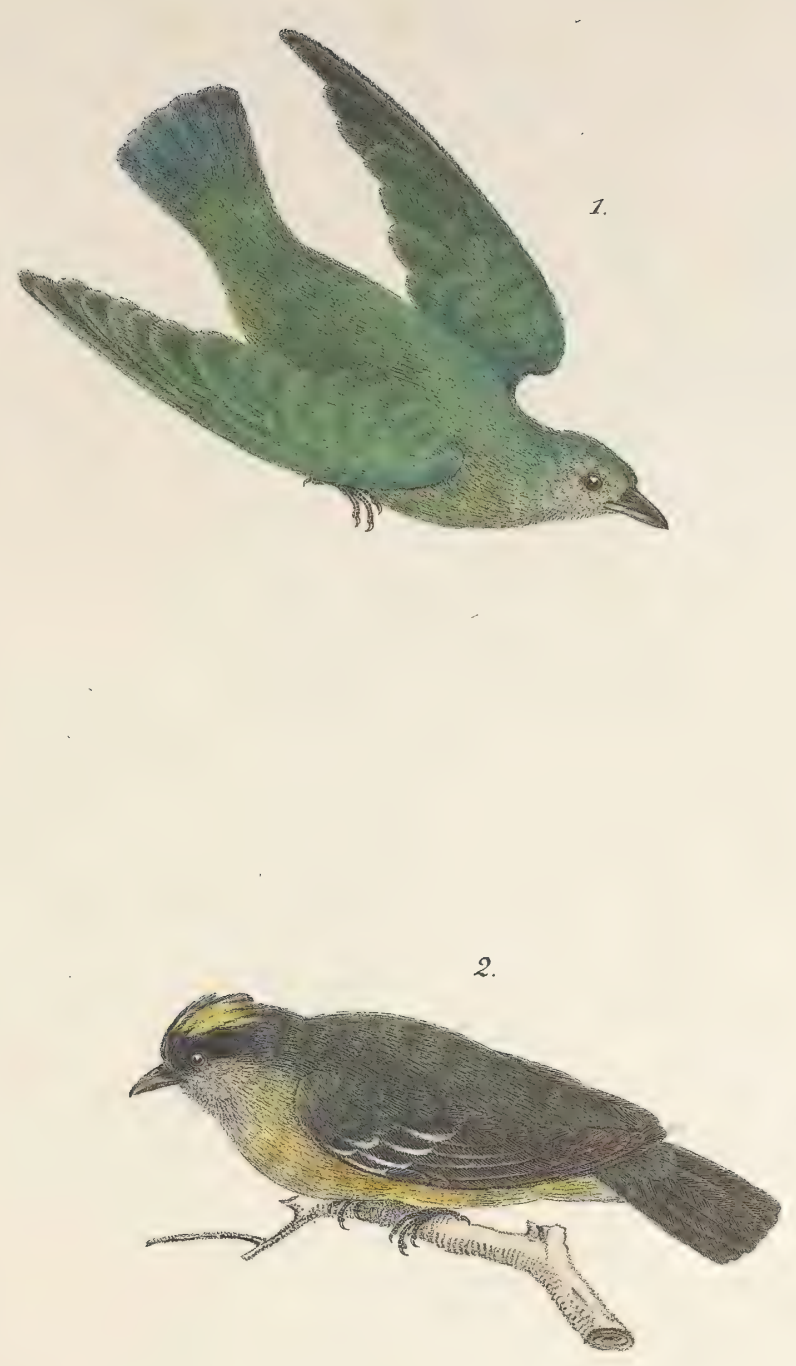

$+\mathbb{P} \mathbb{I P} \mathbb{R} A$ r.herbacea. ${ }^{t}$ elata.

Tab.VIII $\stackrel{a}{.}$ 



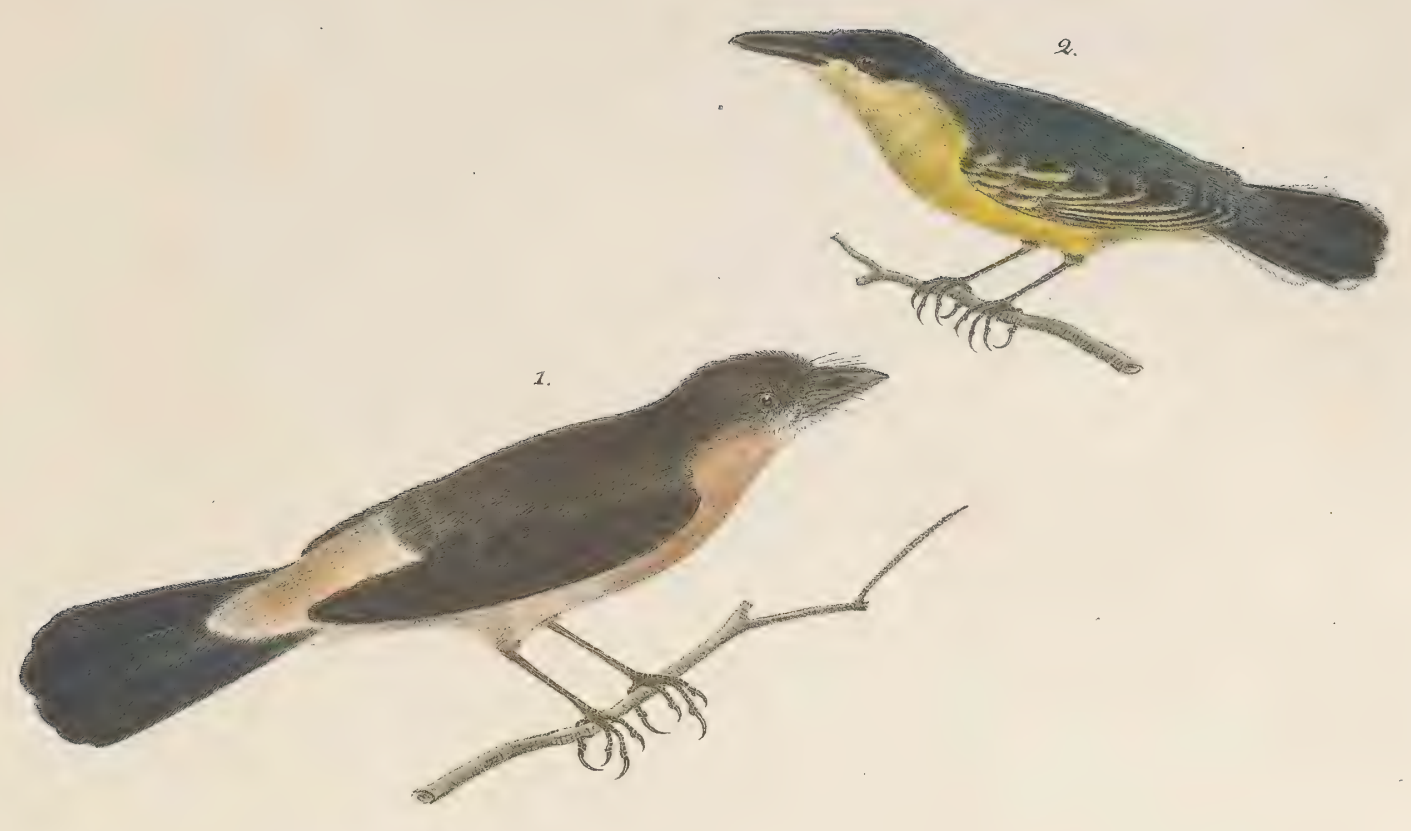

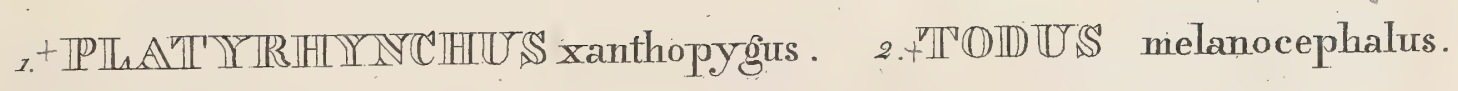

Tab.IX. 





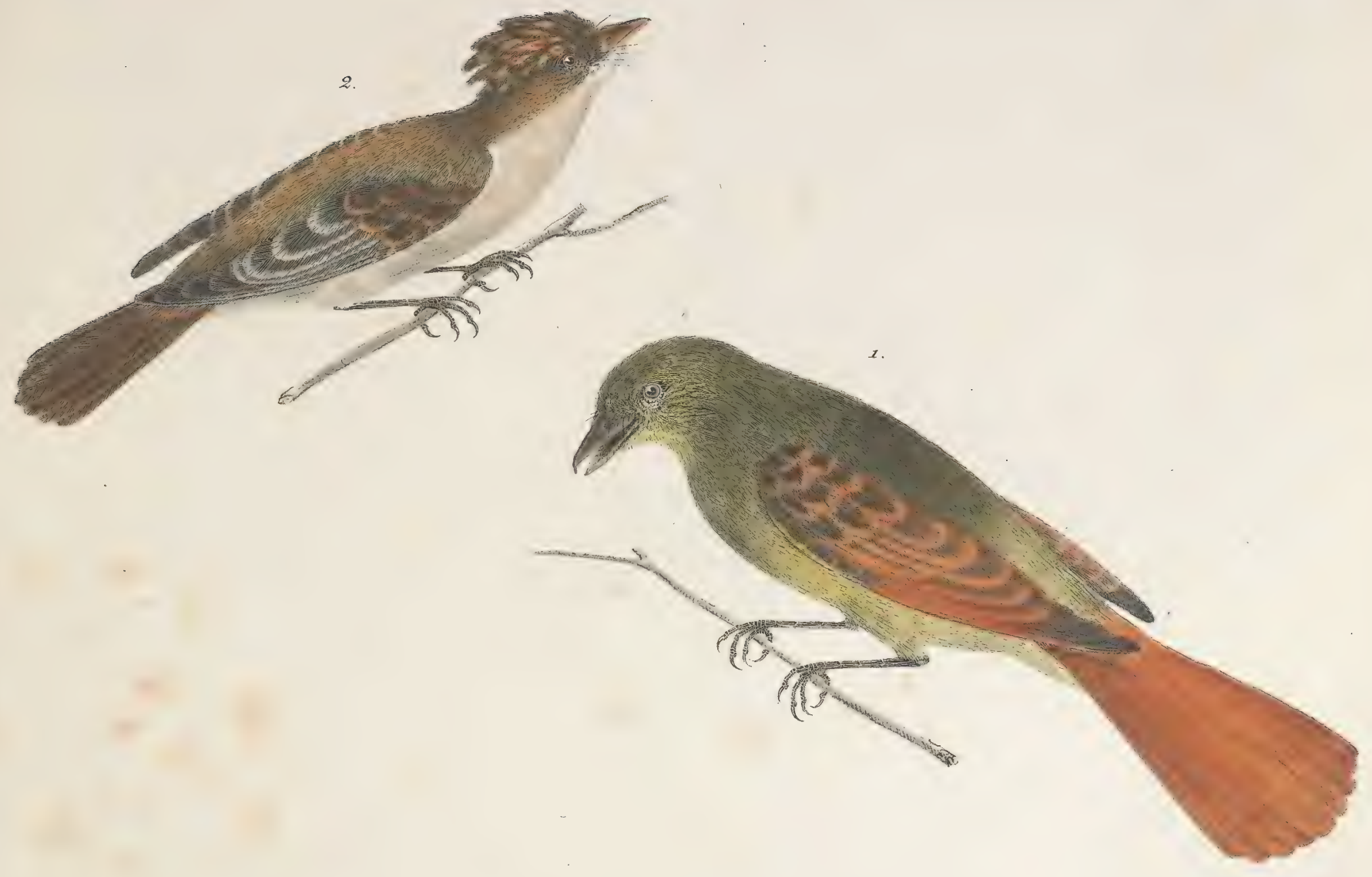

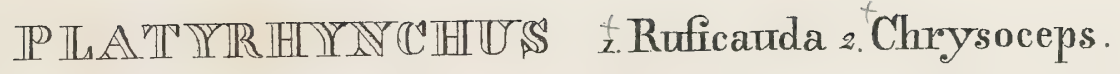

Tab.XI. 


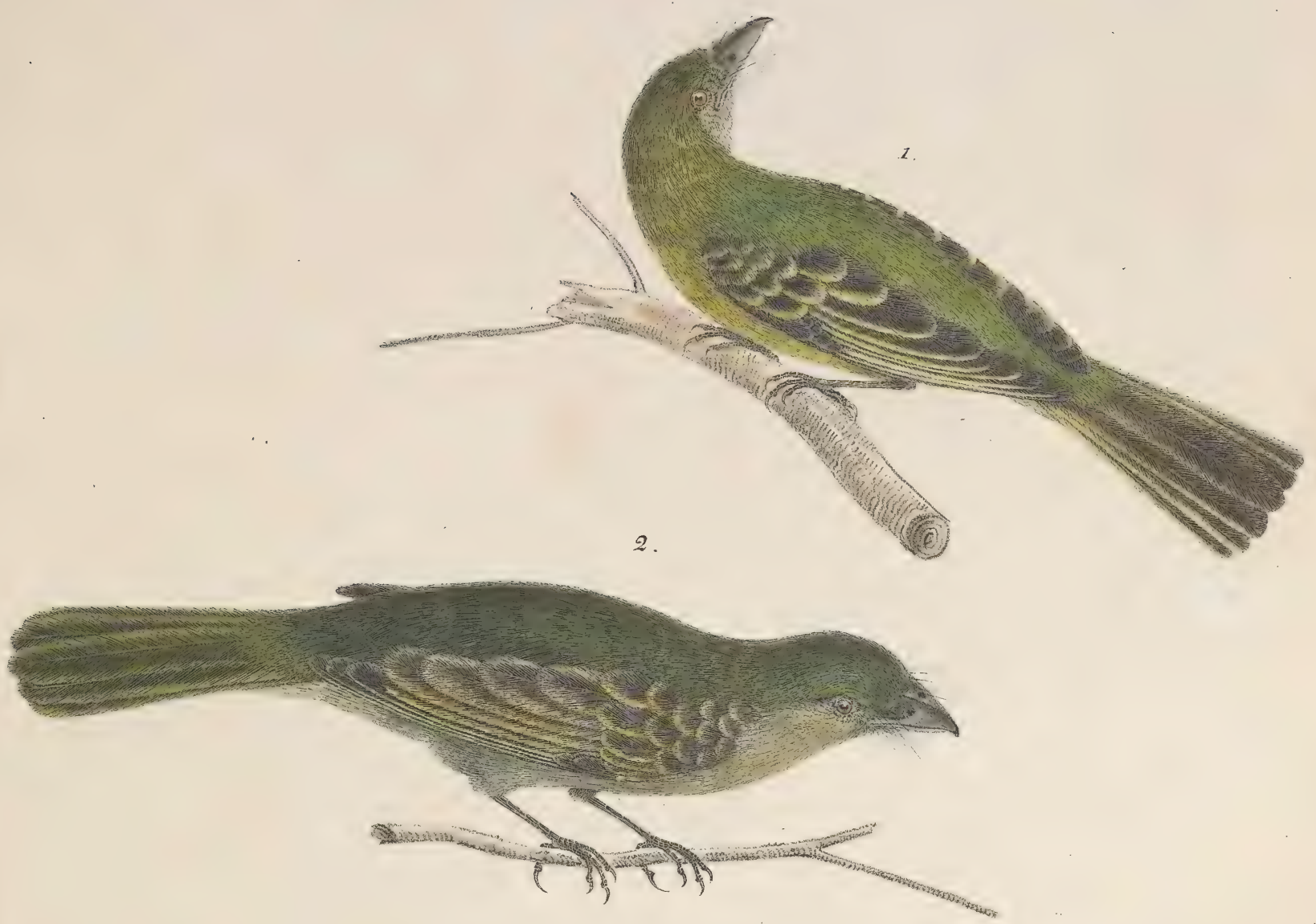

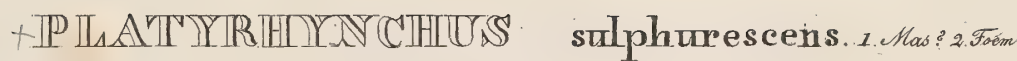

Tab.XII . 


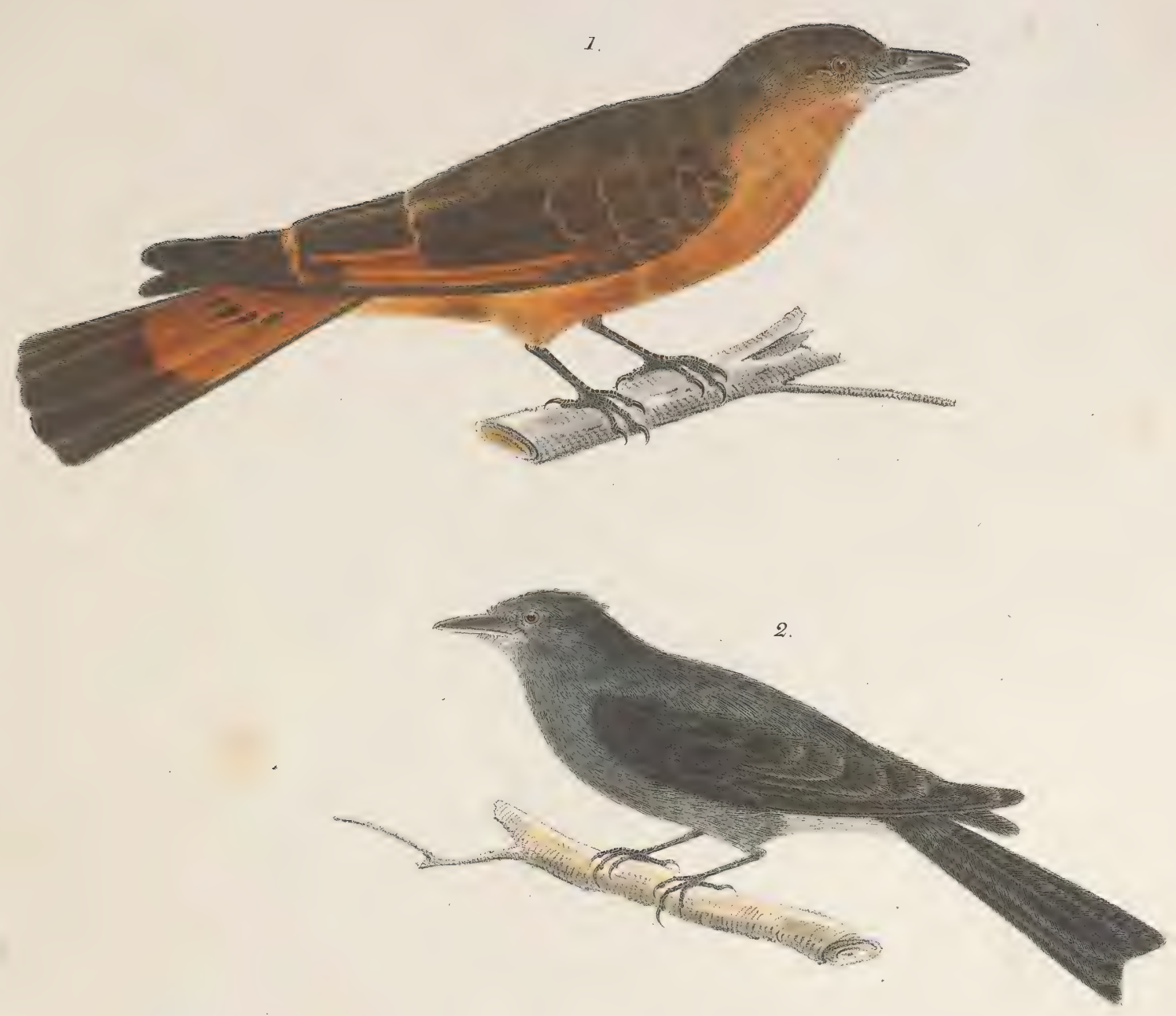

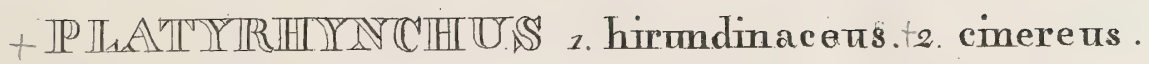

Tab XIII. 


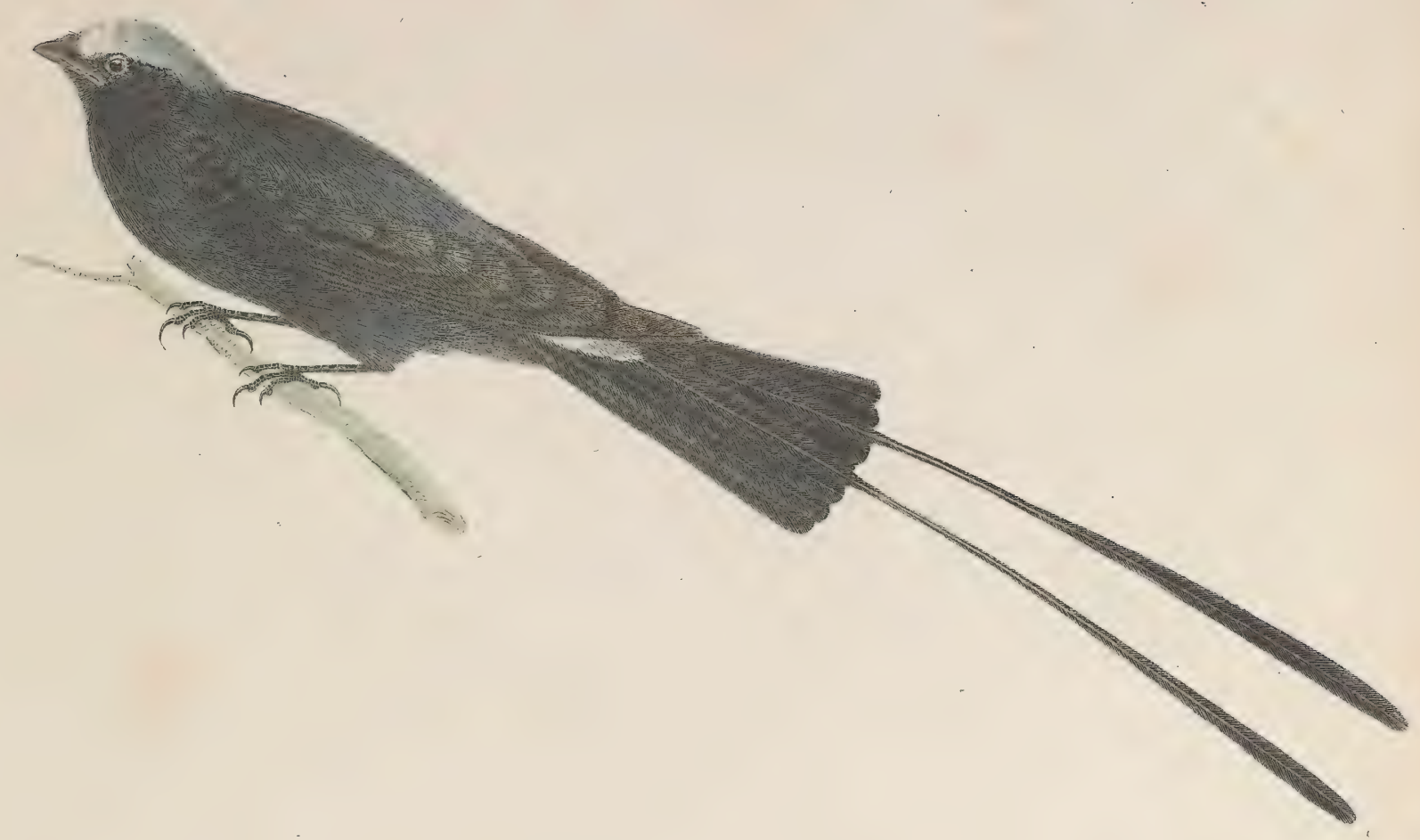

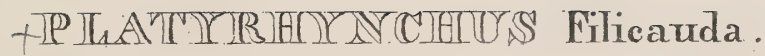

Tab : XIV. 

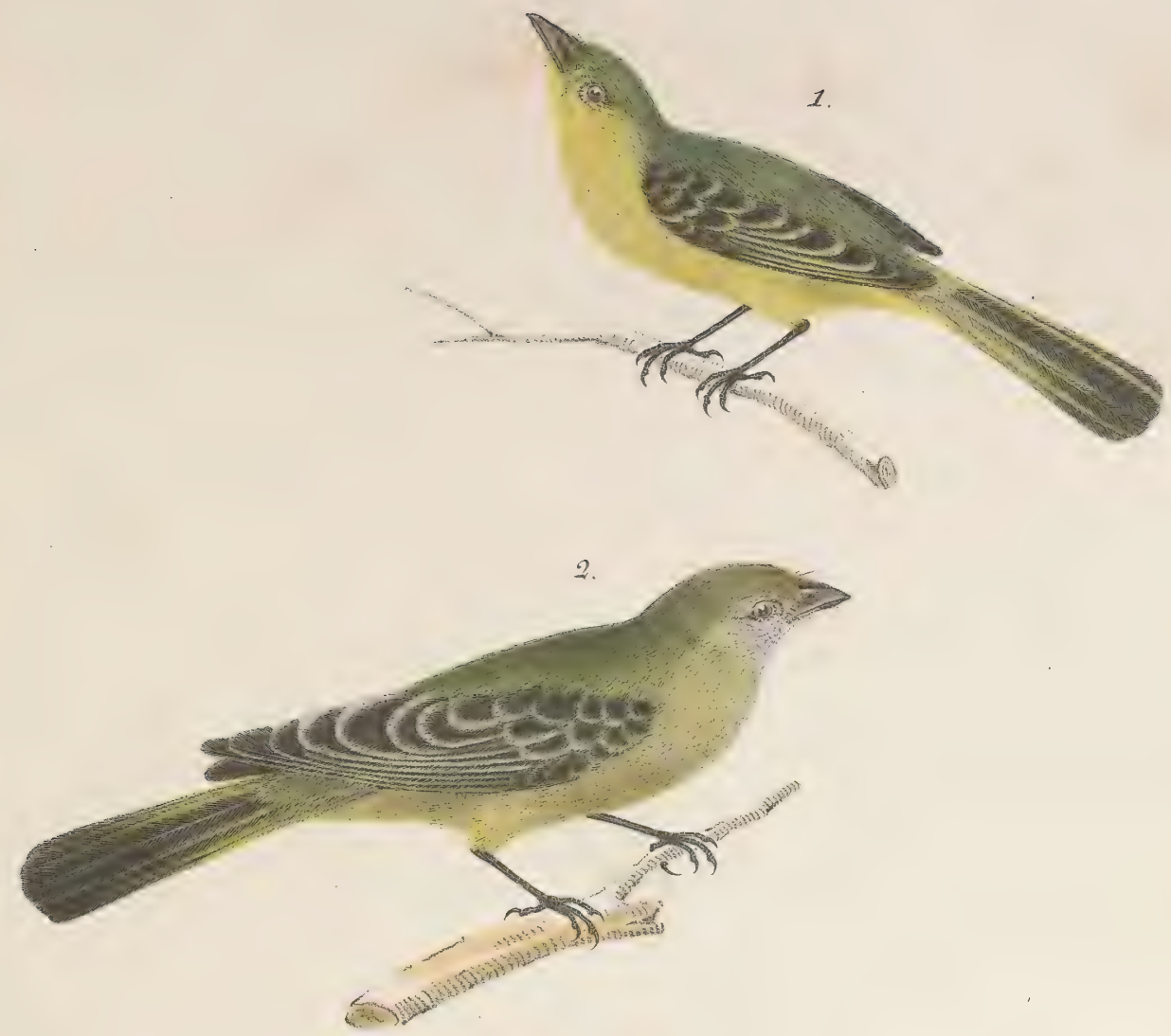

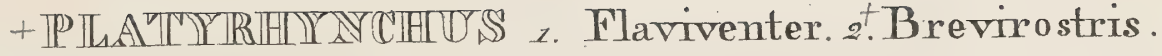

Tab. XV. 
$a^{\prime}$ 

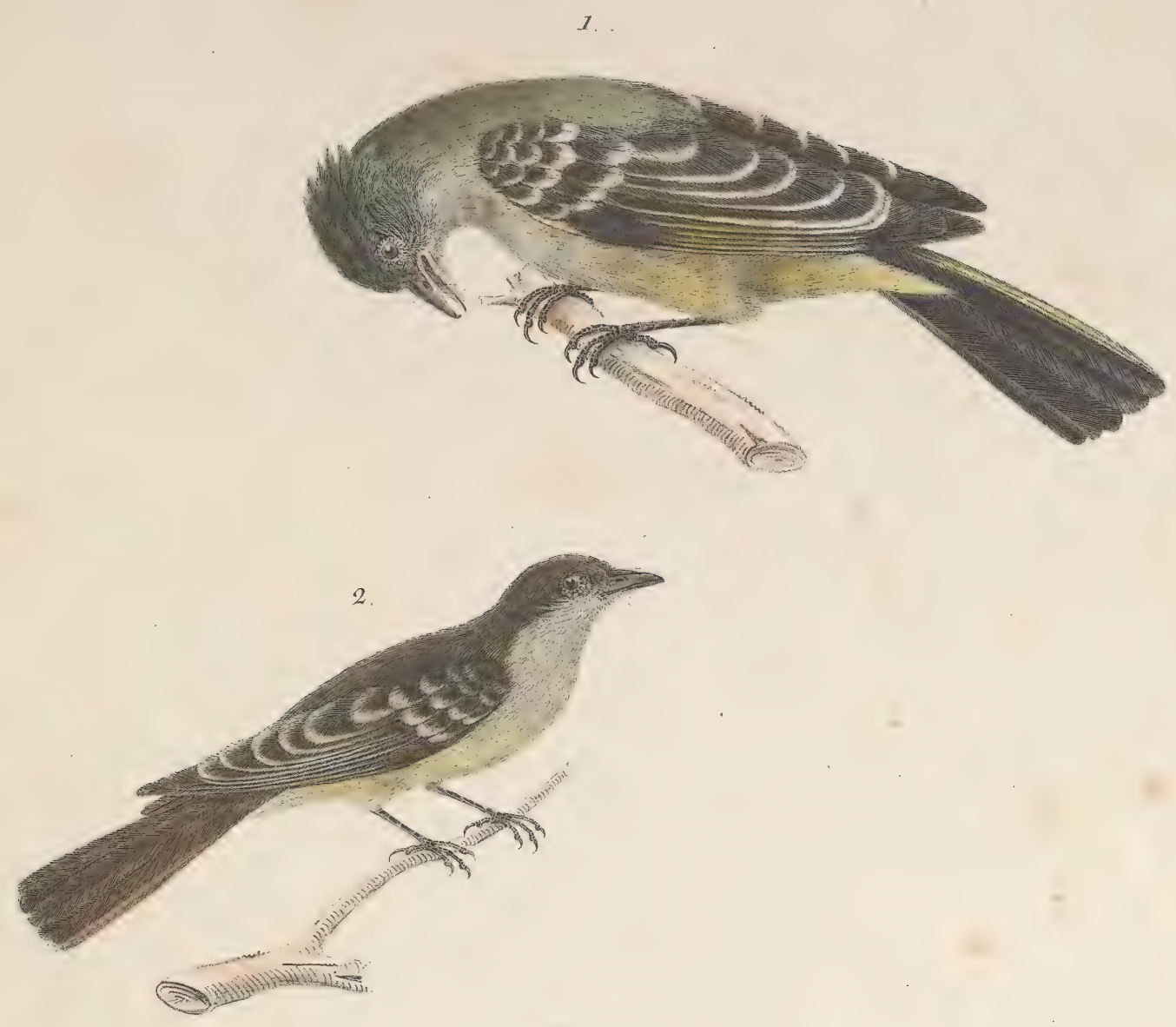

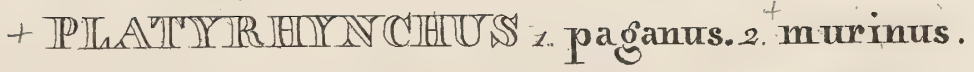

Tab. XVI . 



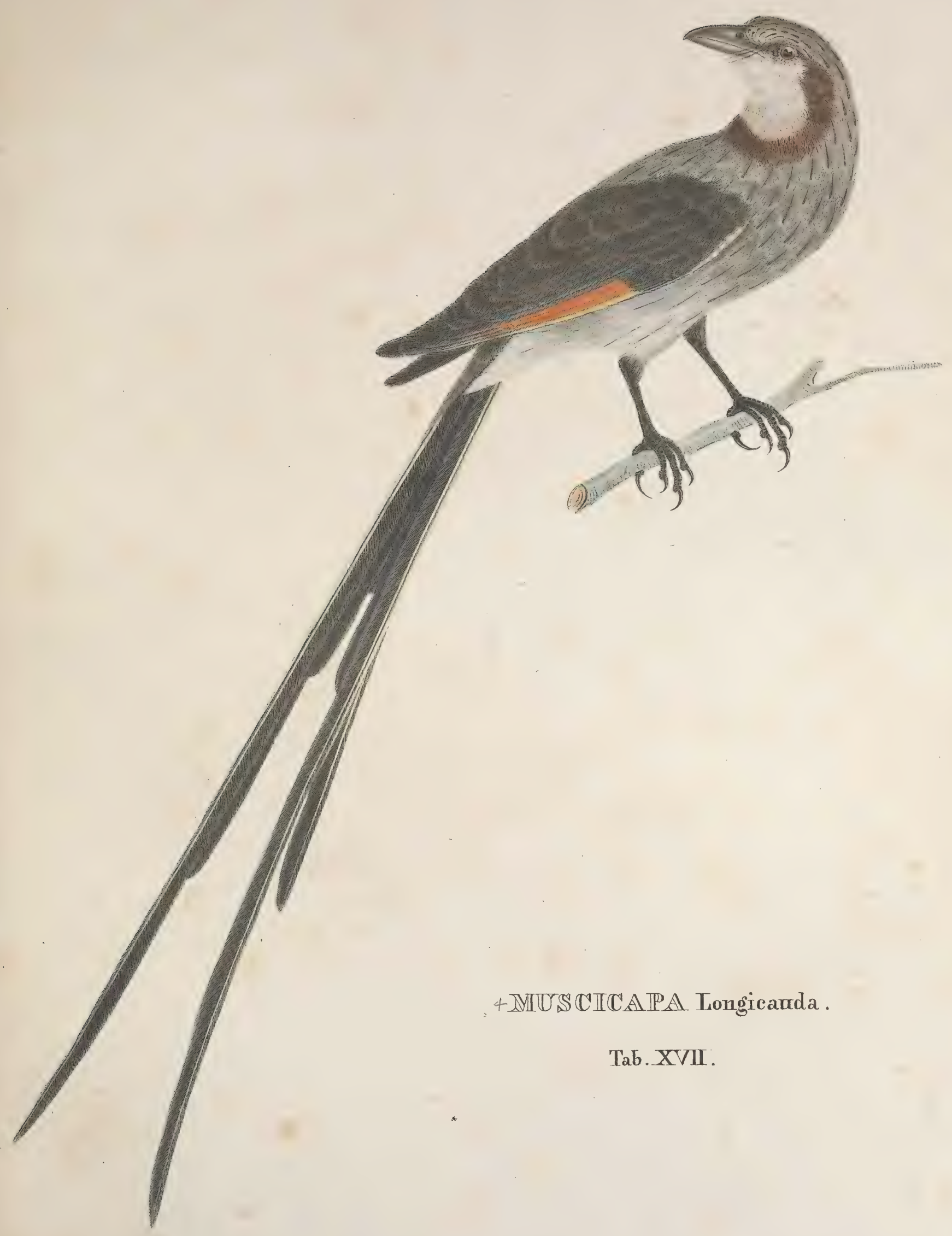




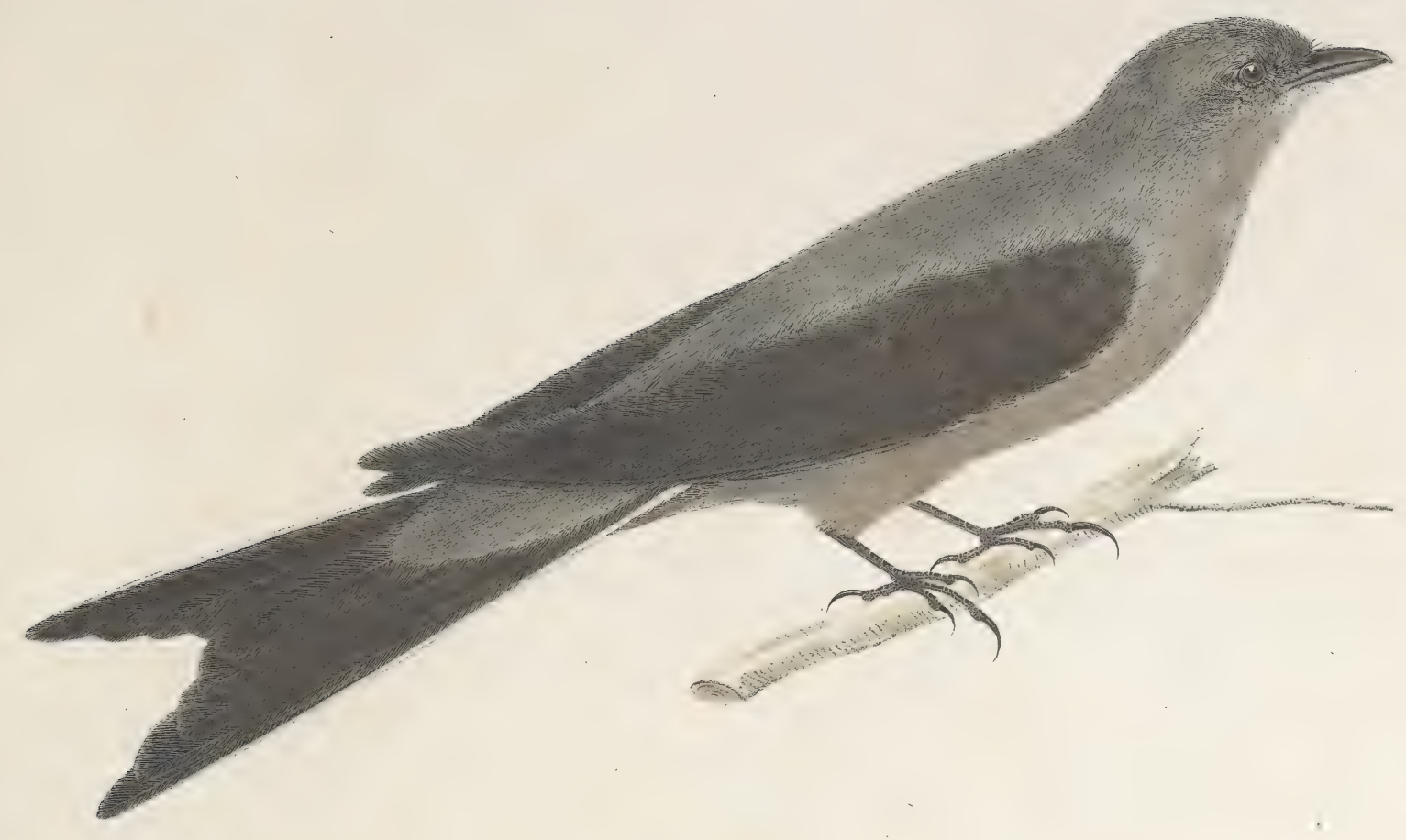

+ MUUS CIICA PA Vetula.

Tab.XVIII. 



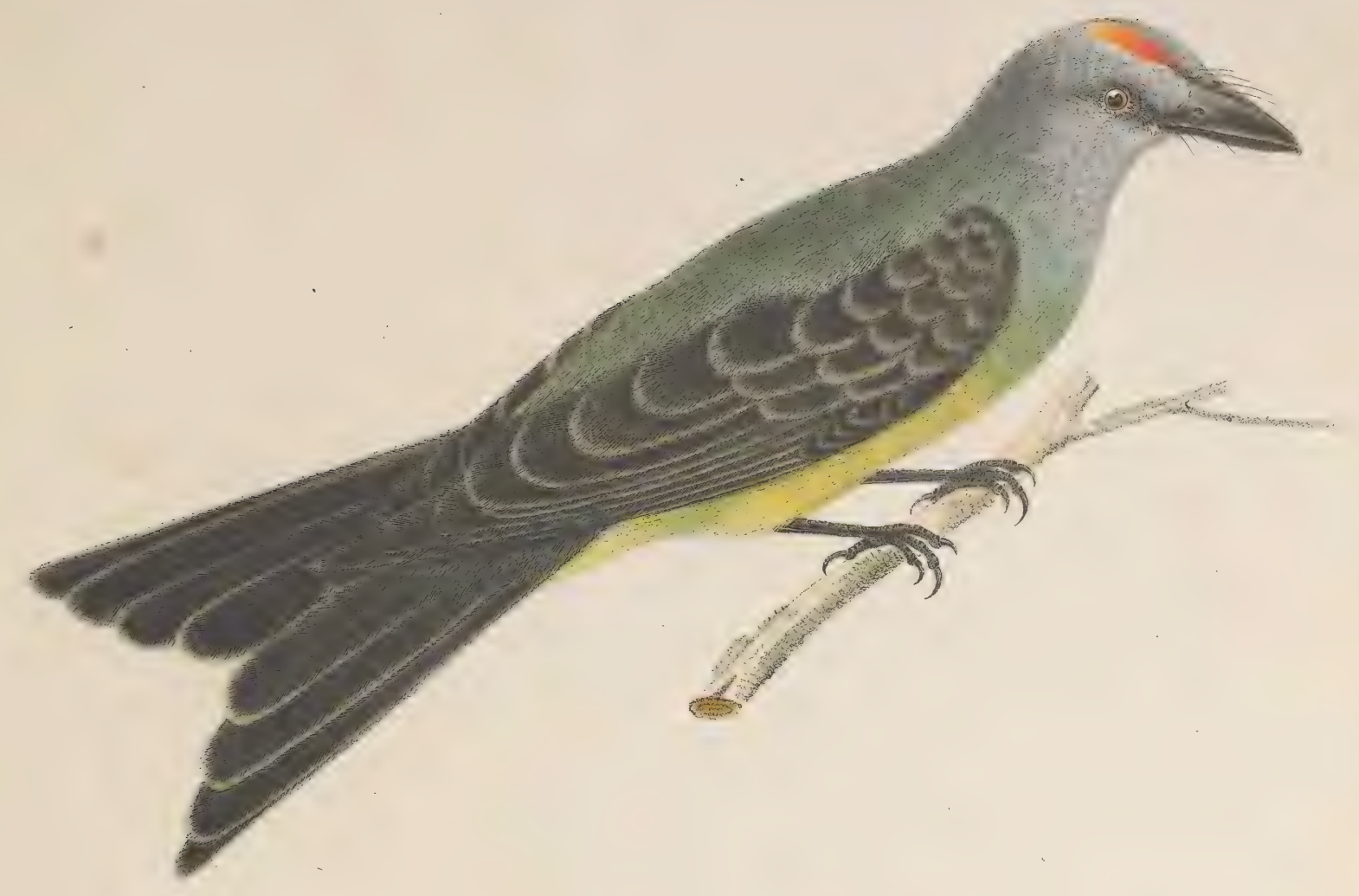

+ MYUSS CIICA APA furcata .

Tab.XIX . 



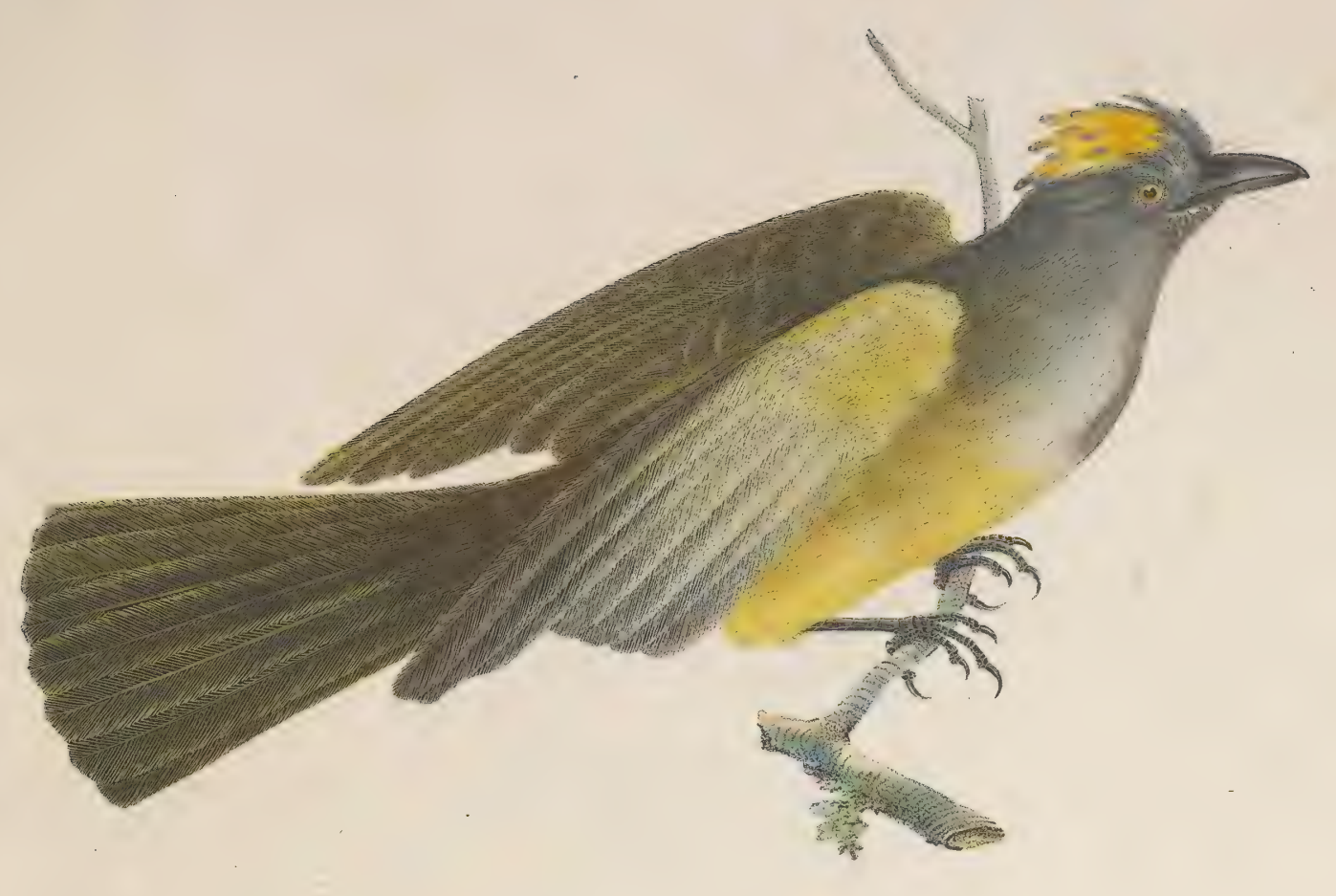

+ MUUSCIICAPA sulphmea

Tab.XX . 





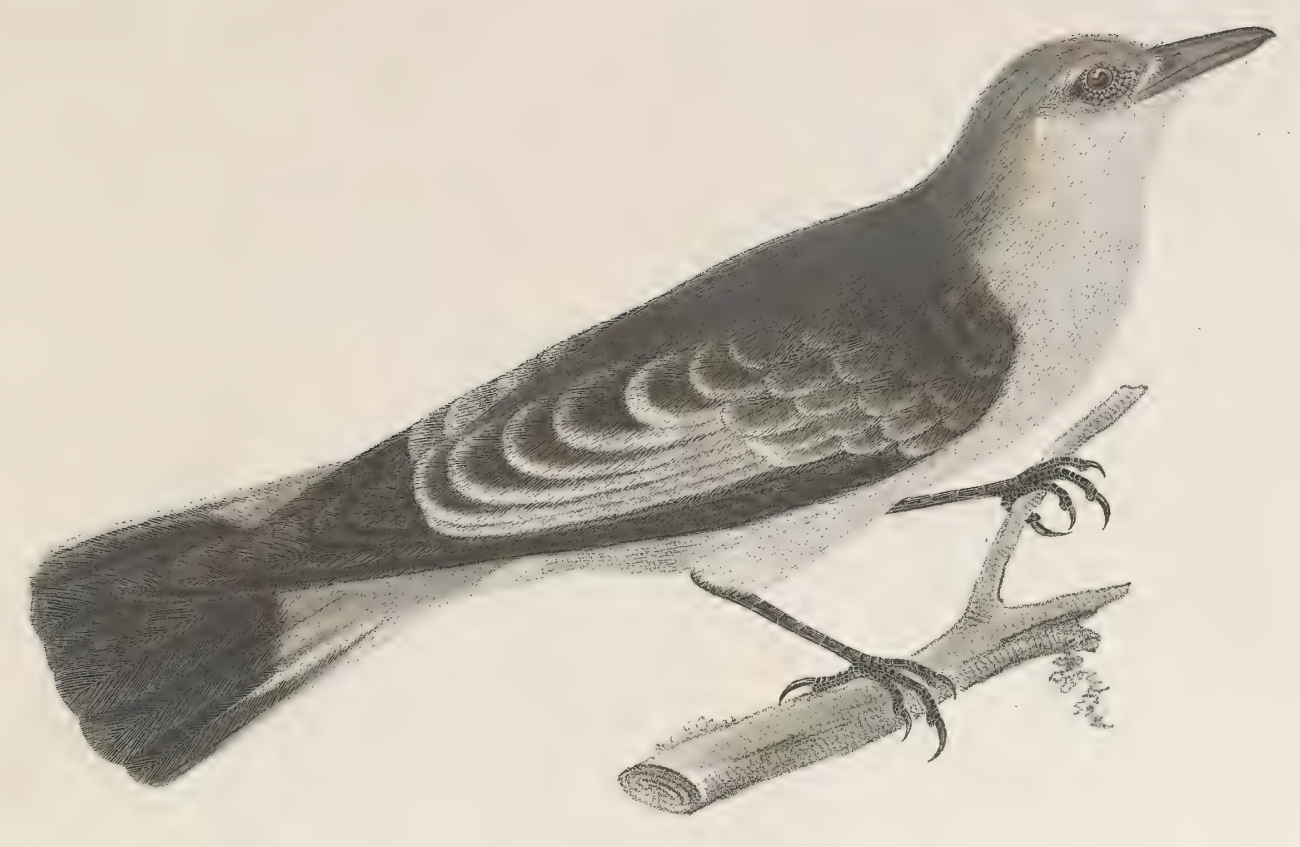

+MUUSCICAPA velata.

Tab. XXII . 



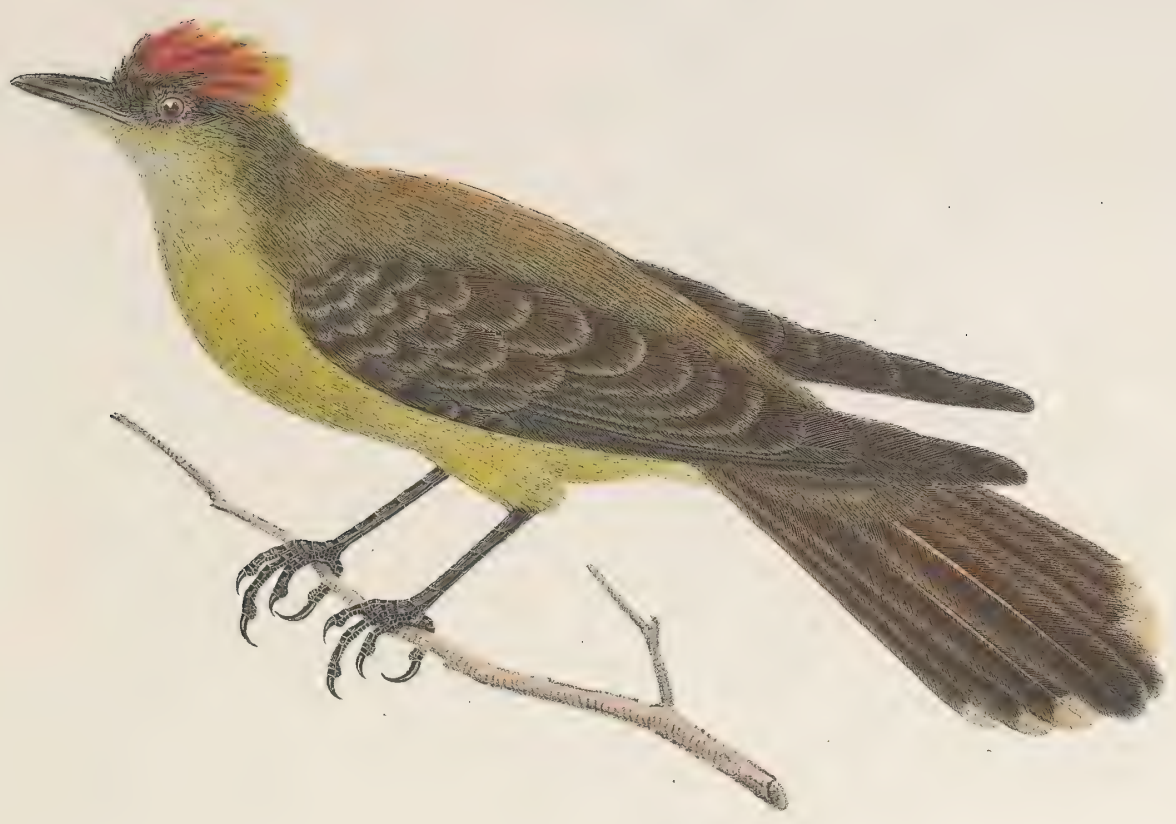

+MUUSCCICAPA Joazeiro.

Tab: XXIII . 



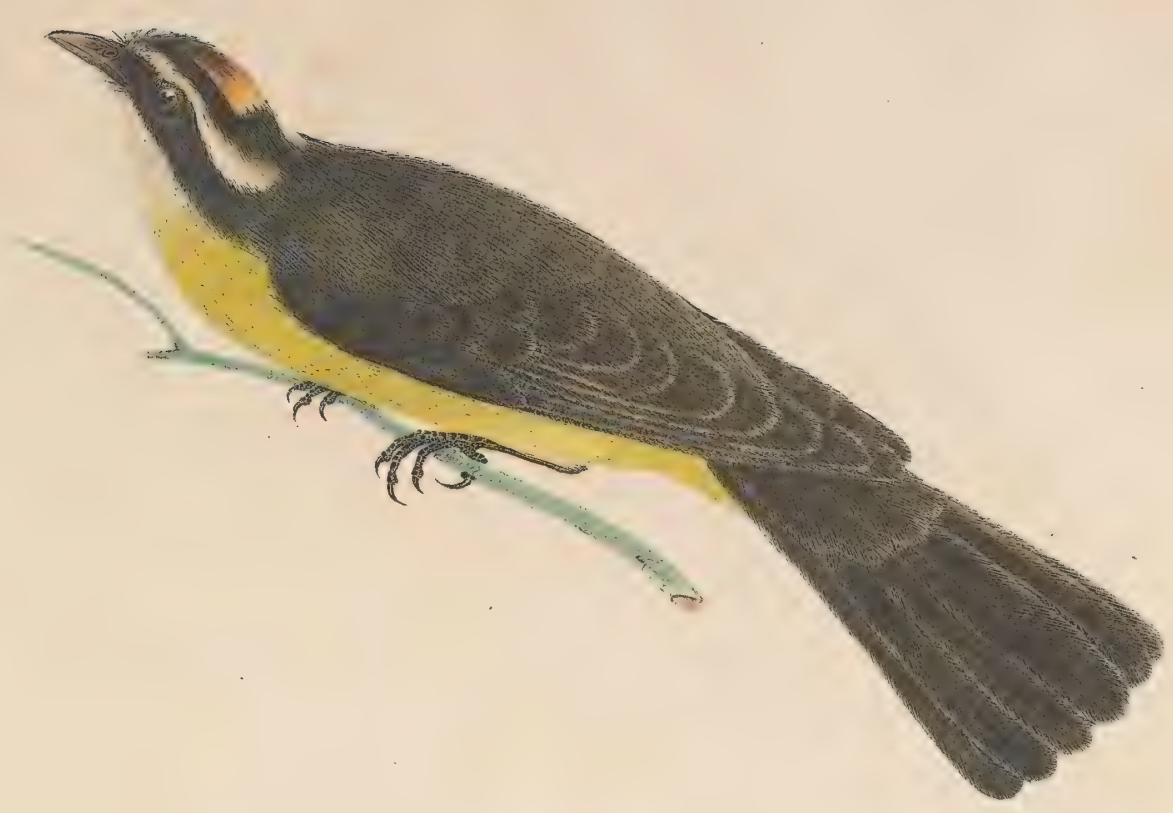

4 MIUSSCIICA PA similis .

Tab.XXV. 



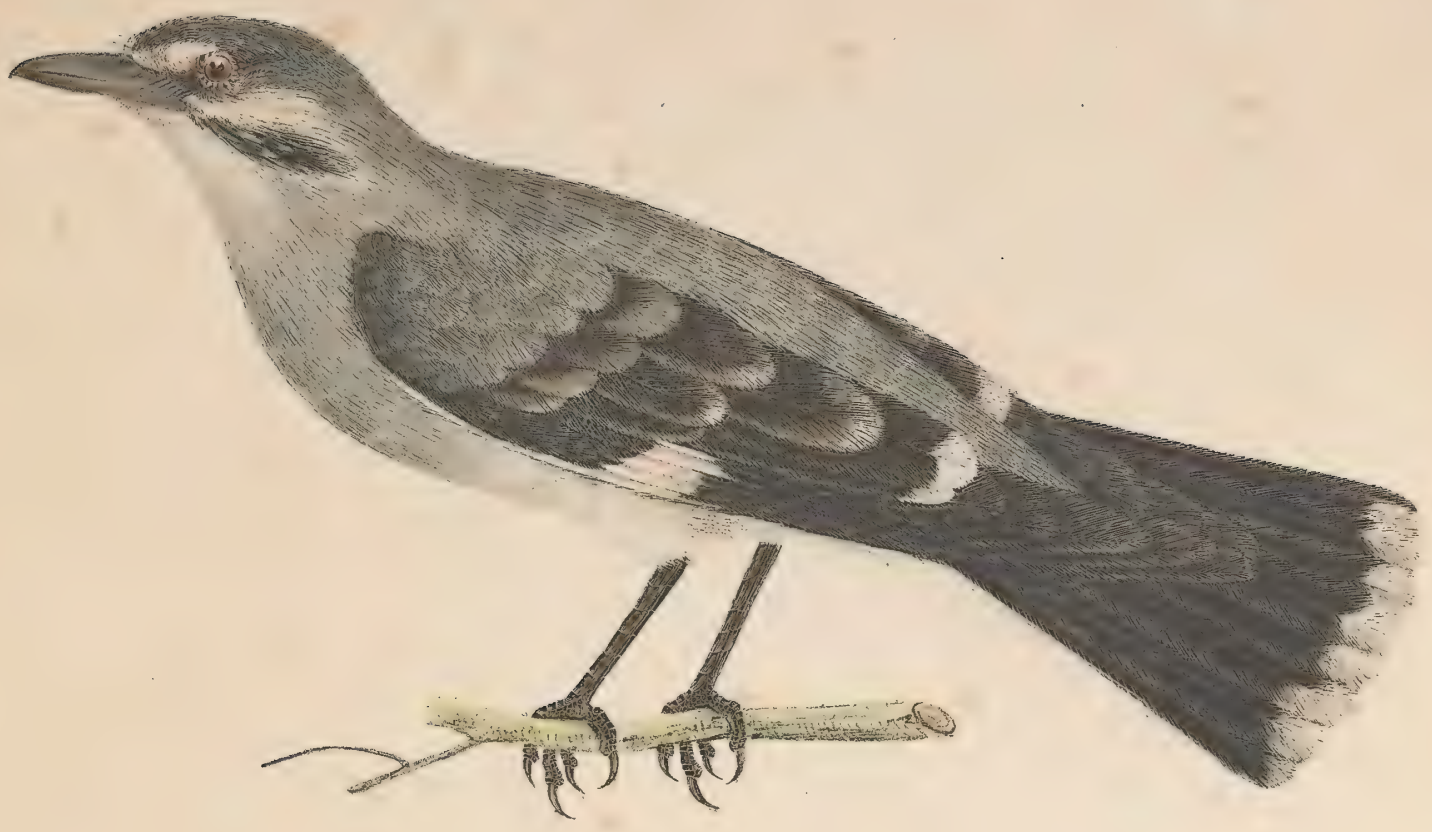

+ MUS CICAPA Polyglotta.

Tab.XXIV. 



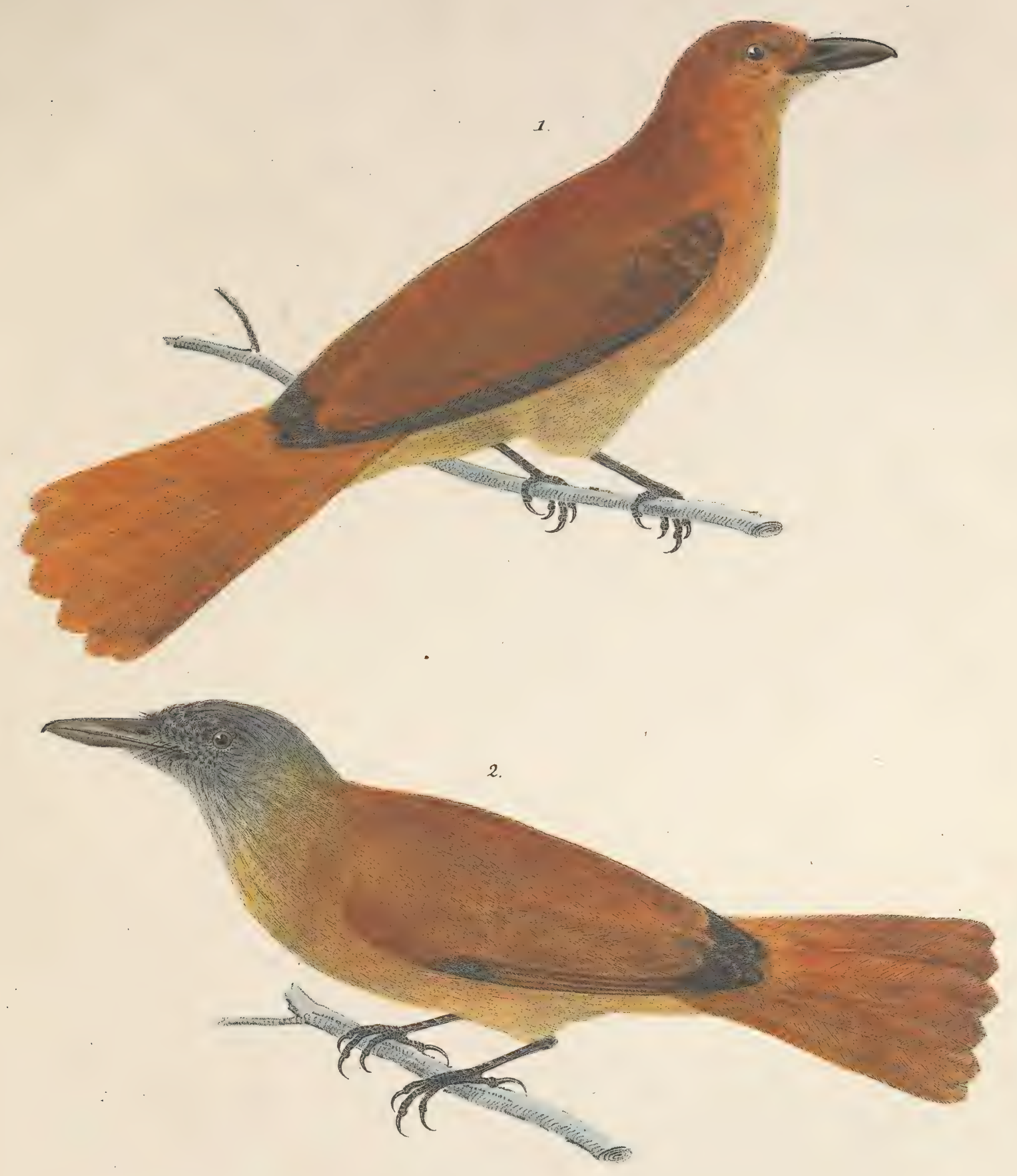

MVIES CCIC A PA , thamnophiloides. 2. cinerea .

Tab. XXVI . 



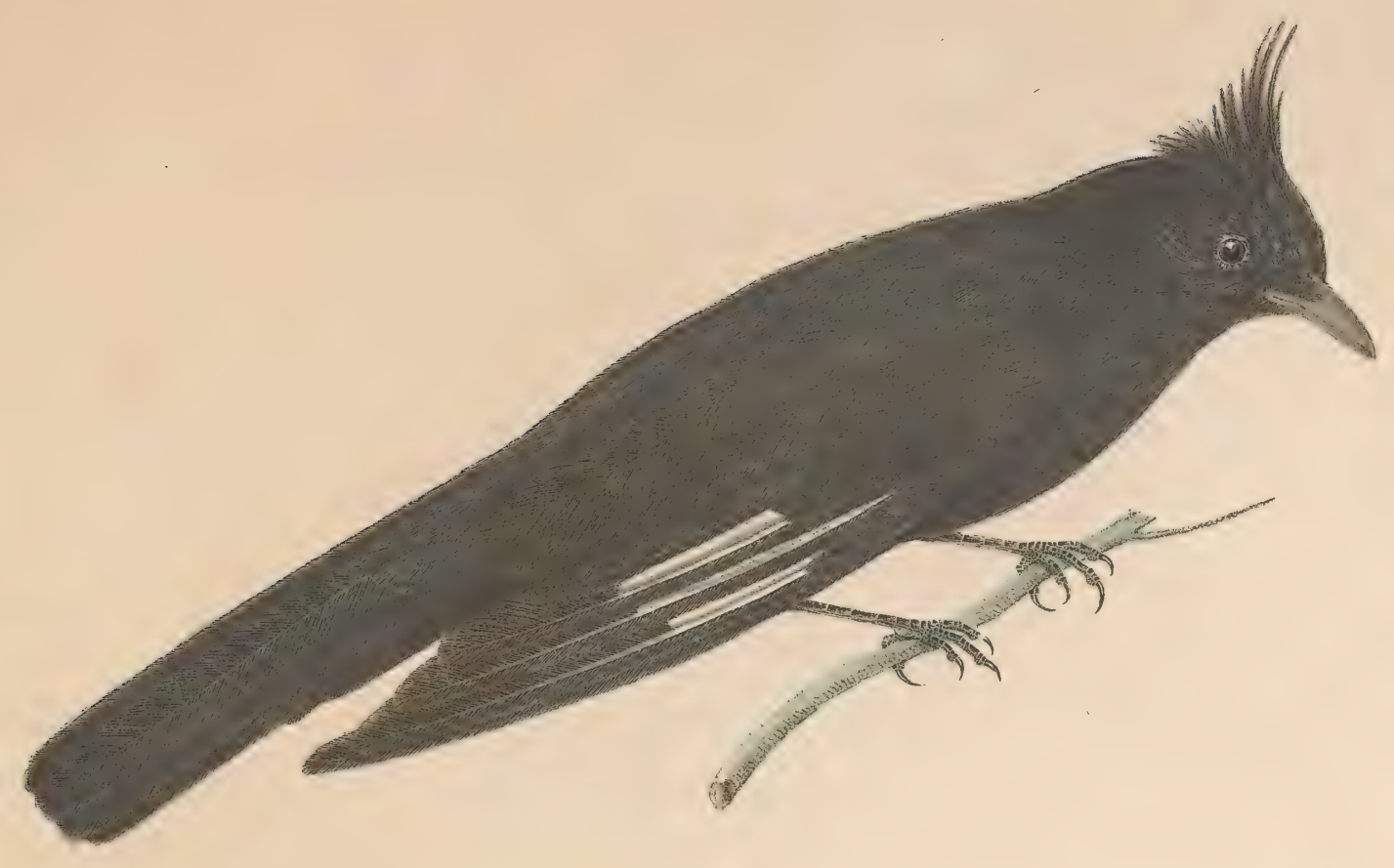

+ MUUSCIICAPA galeata.

Tab.XXVII . 


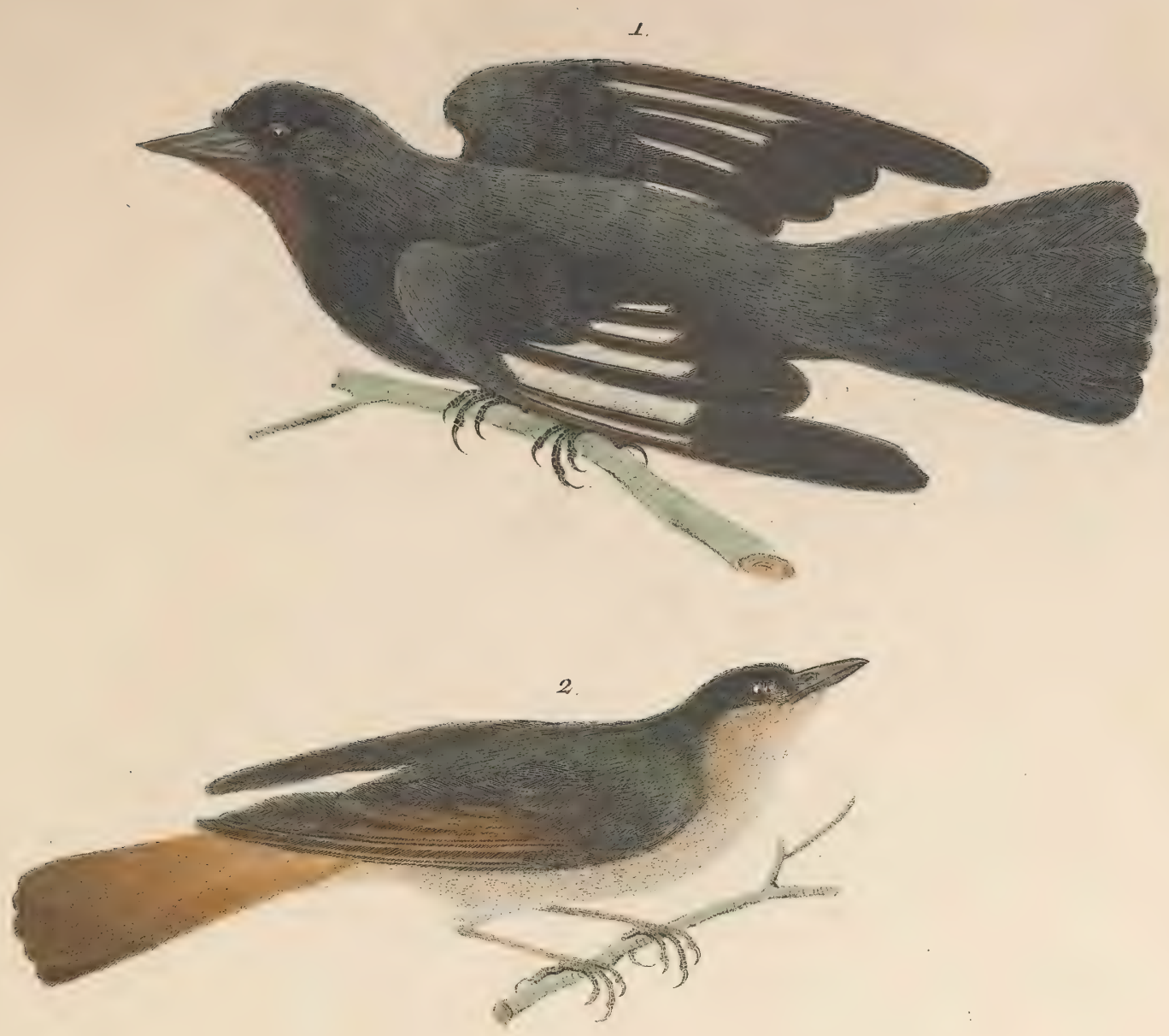

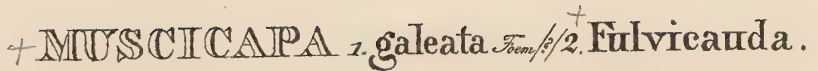

Tab.XXVIII . 

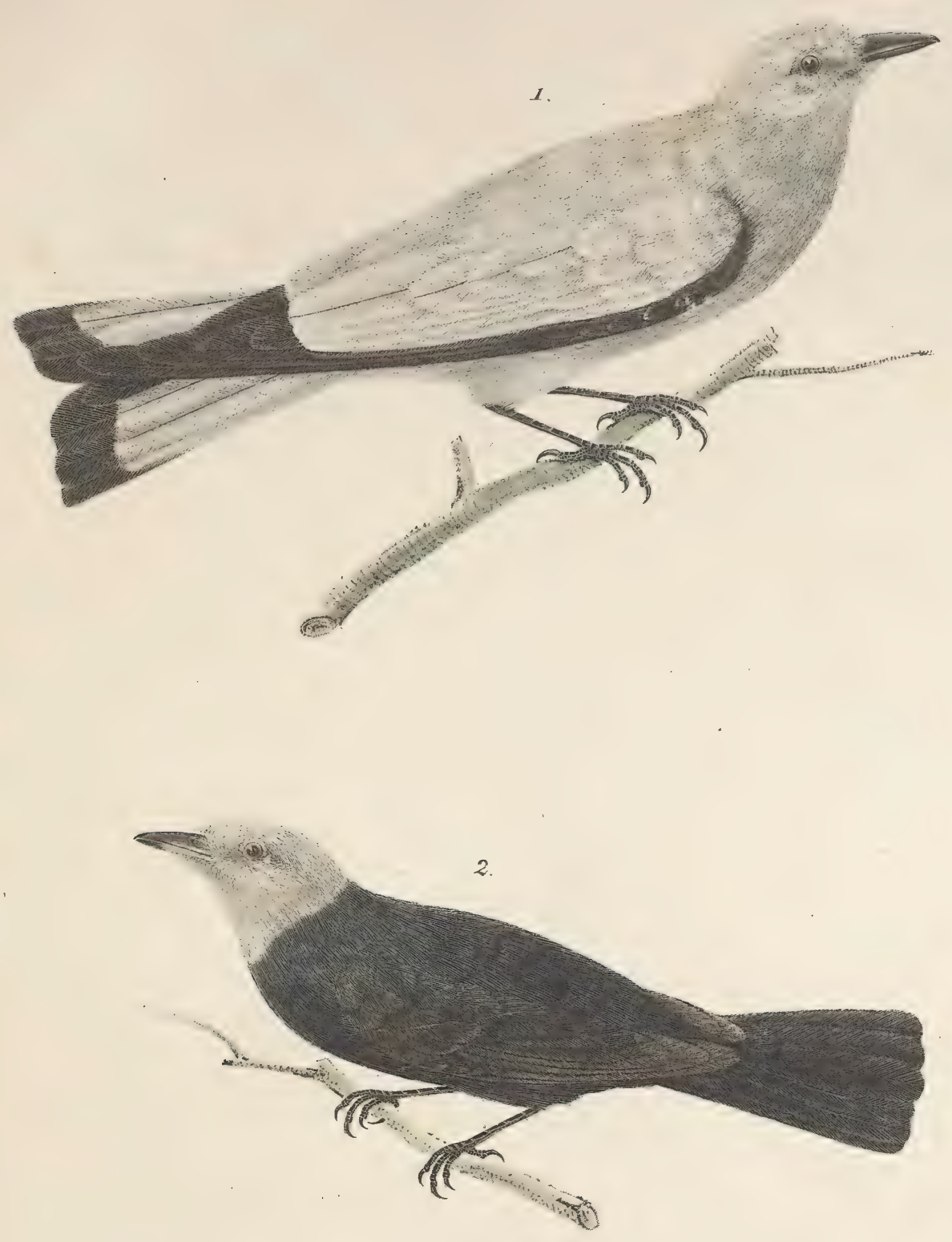

$+\mathbb{M U S} \mathbb{C} \mathbb{C I C A} \mathbb{P A}$ 1 nivea. 2. Dominicana .

Táb.XXIX. 



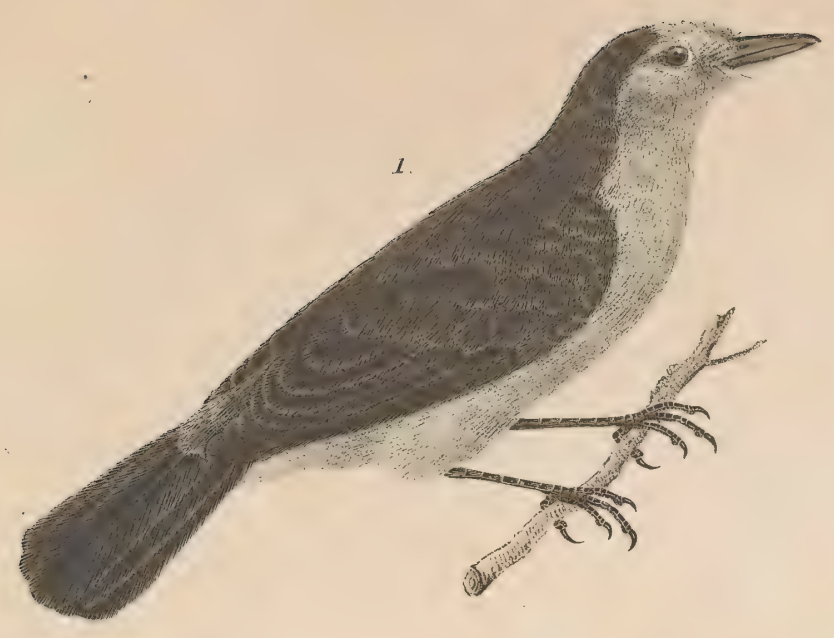

2.

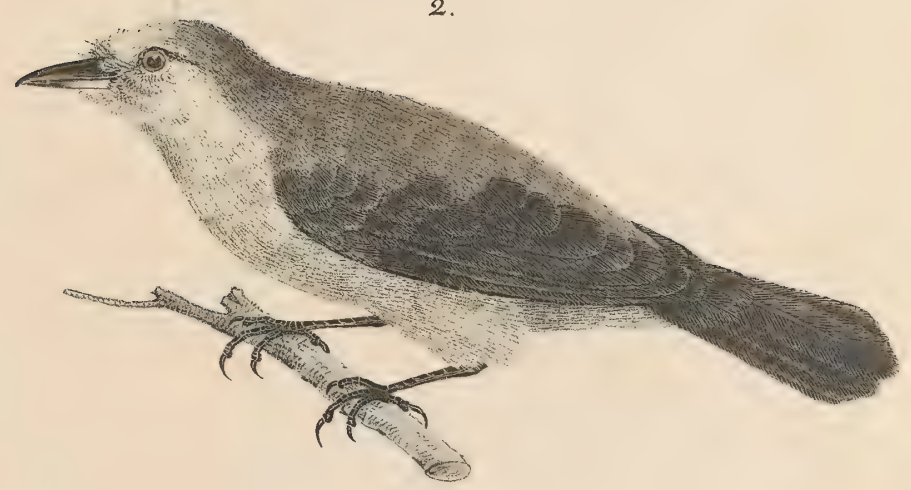

$+\mathbb{V} \mathbb{U} \mathbb{S} \mathbb{C} I \mathbb{C} \mathbb{A P}_{1}$ Albiventer. ${ }^{+}$dominicana. F

Tab.XXX . 



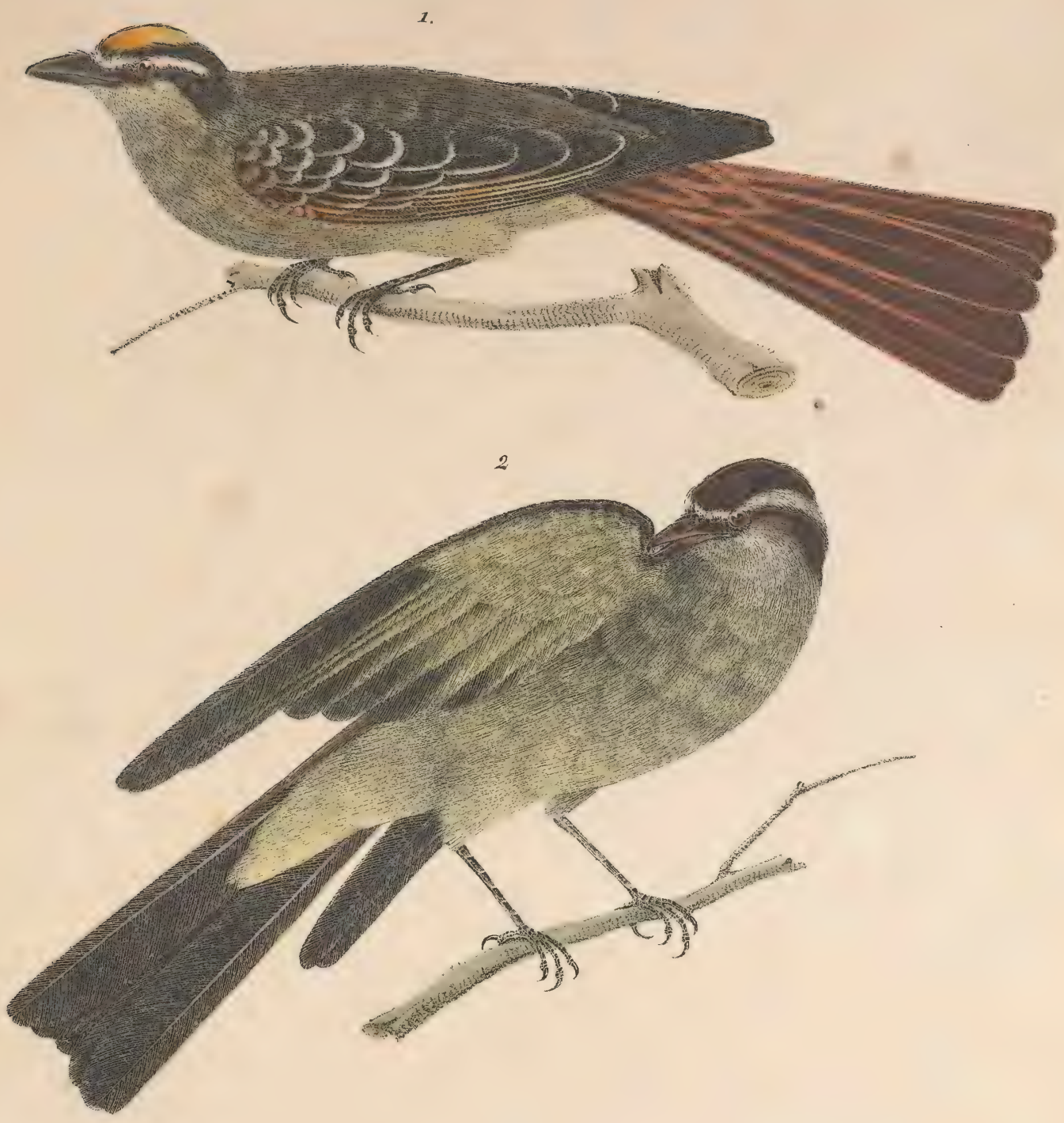

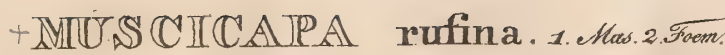

Tab. XXXI. 

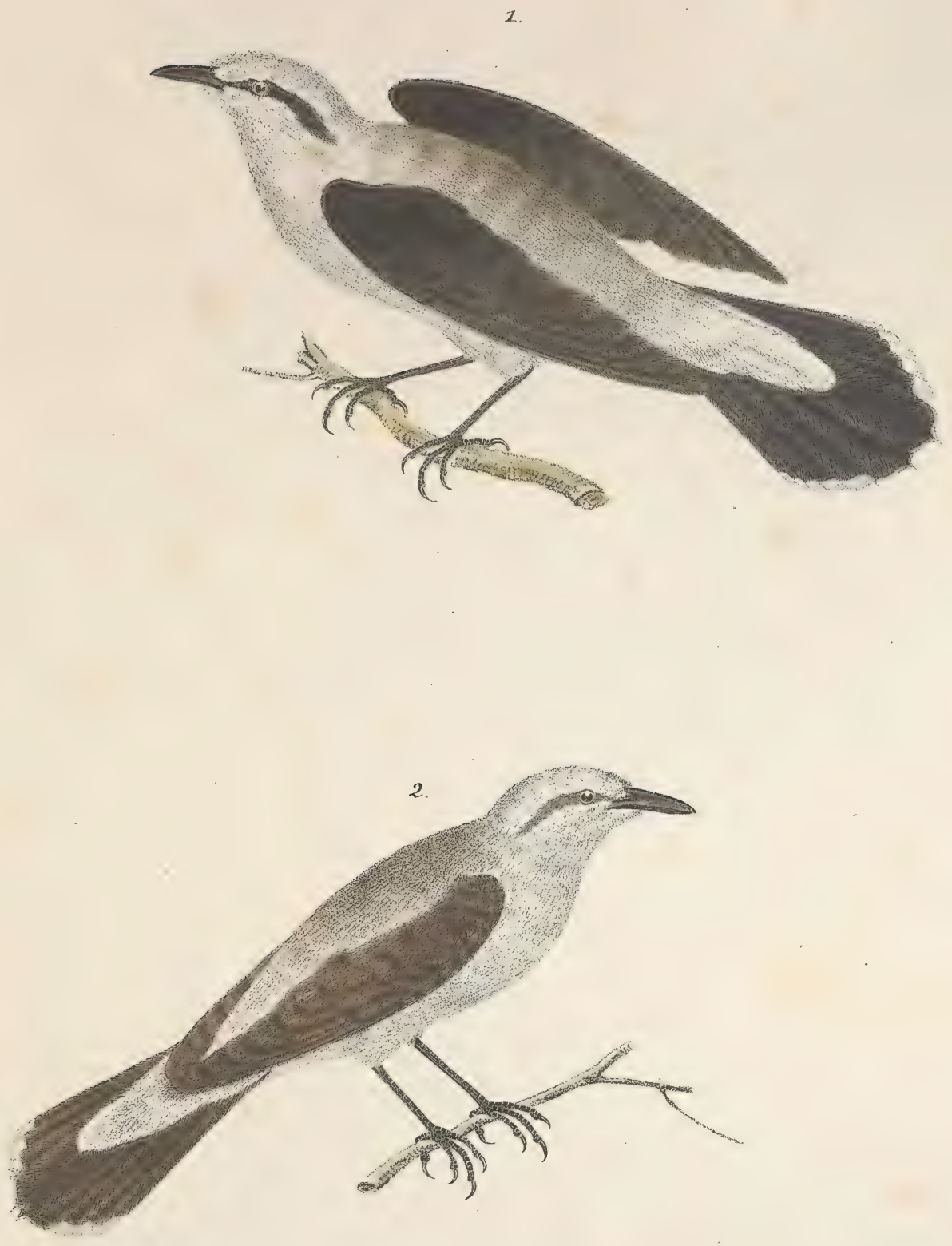

+ MUSS CICA PA Mystax. 1.K. 2.7.

Tab. XXXI 



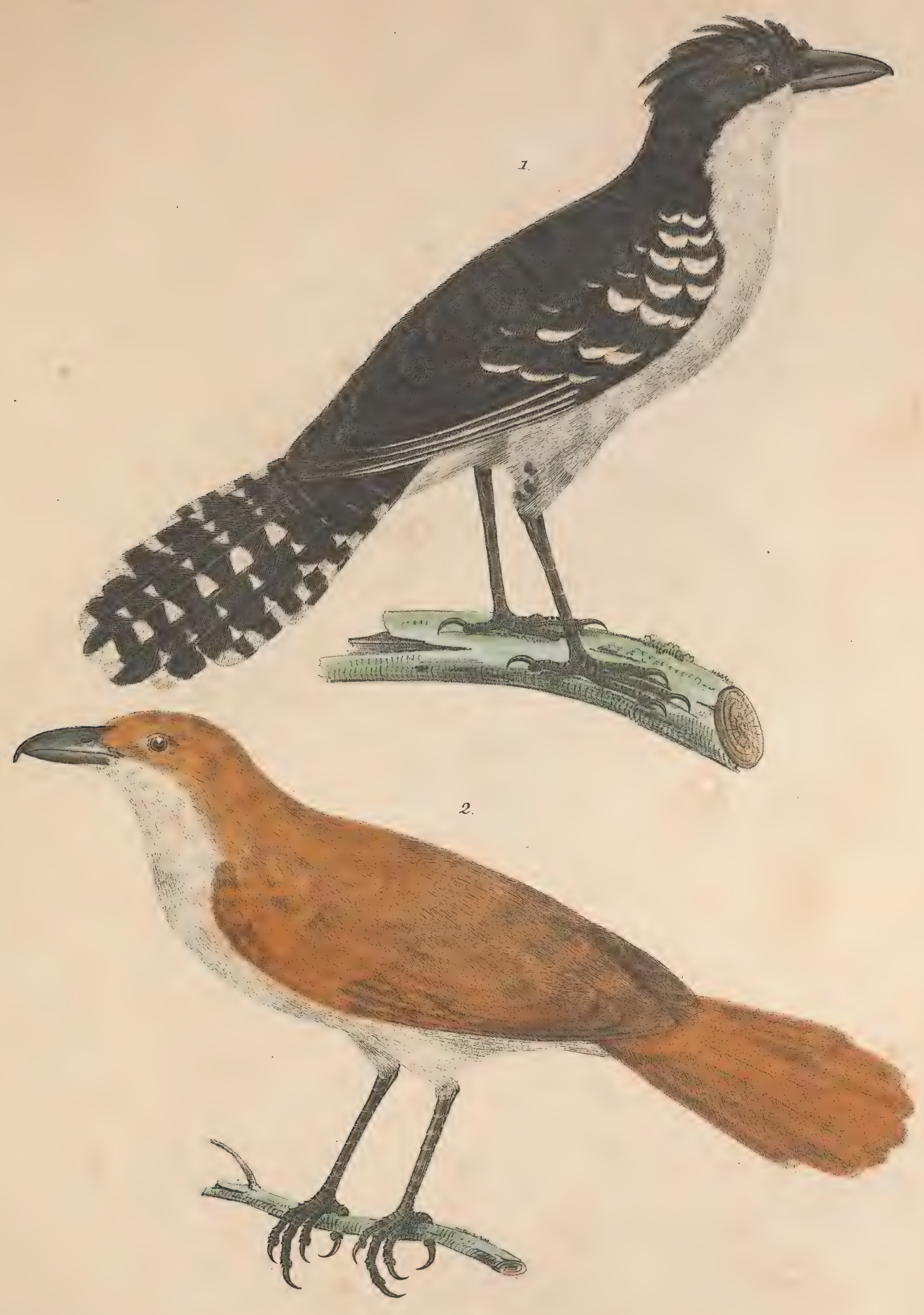

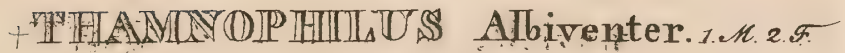

Tab.XXXII . 



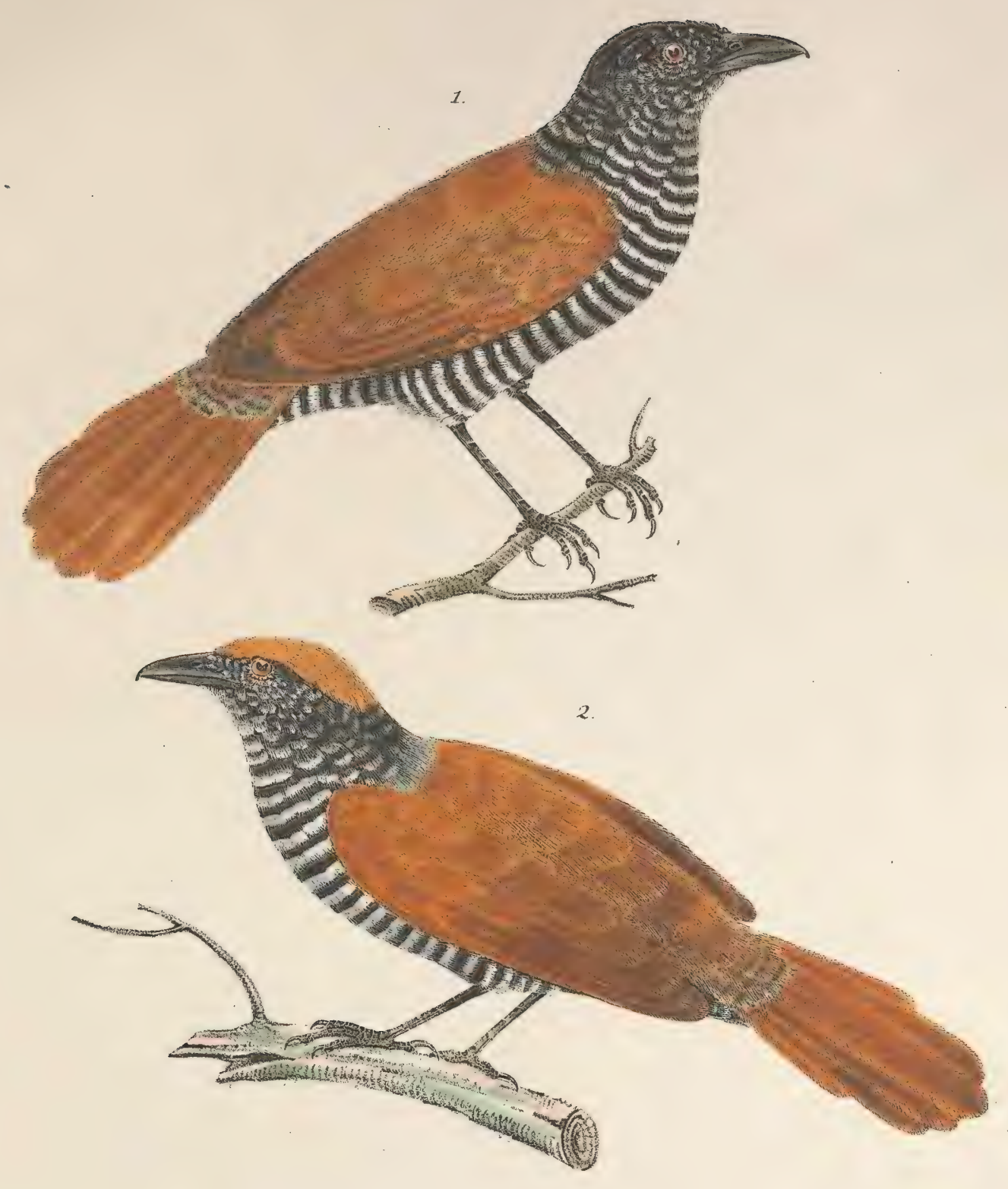

+THEAMINOPIHILUS lineatus. 7. Mas. 2. Toom:

Tab.XXXIII . 


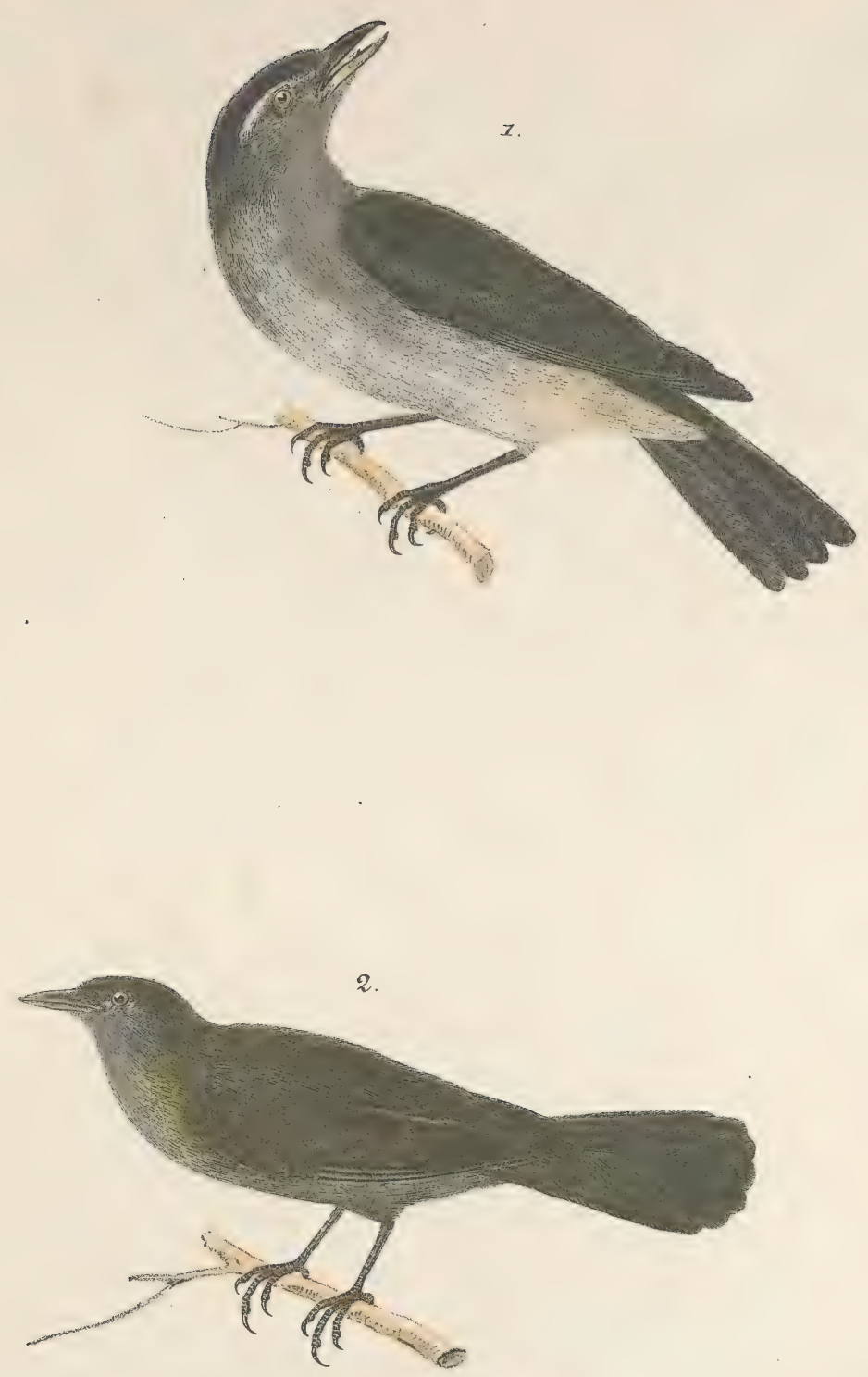

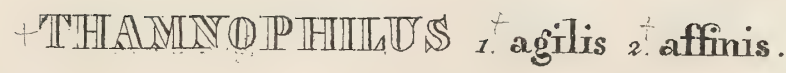

Tab. XXXIV. 


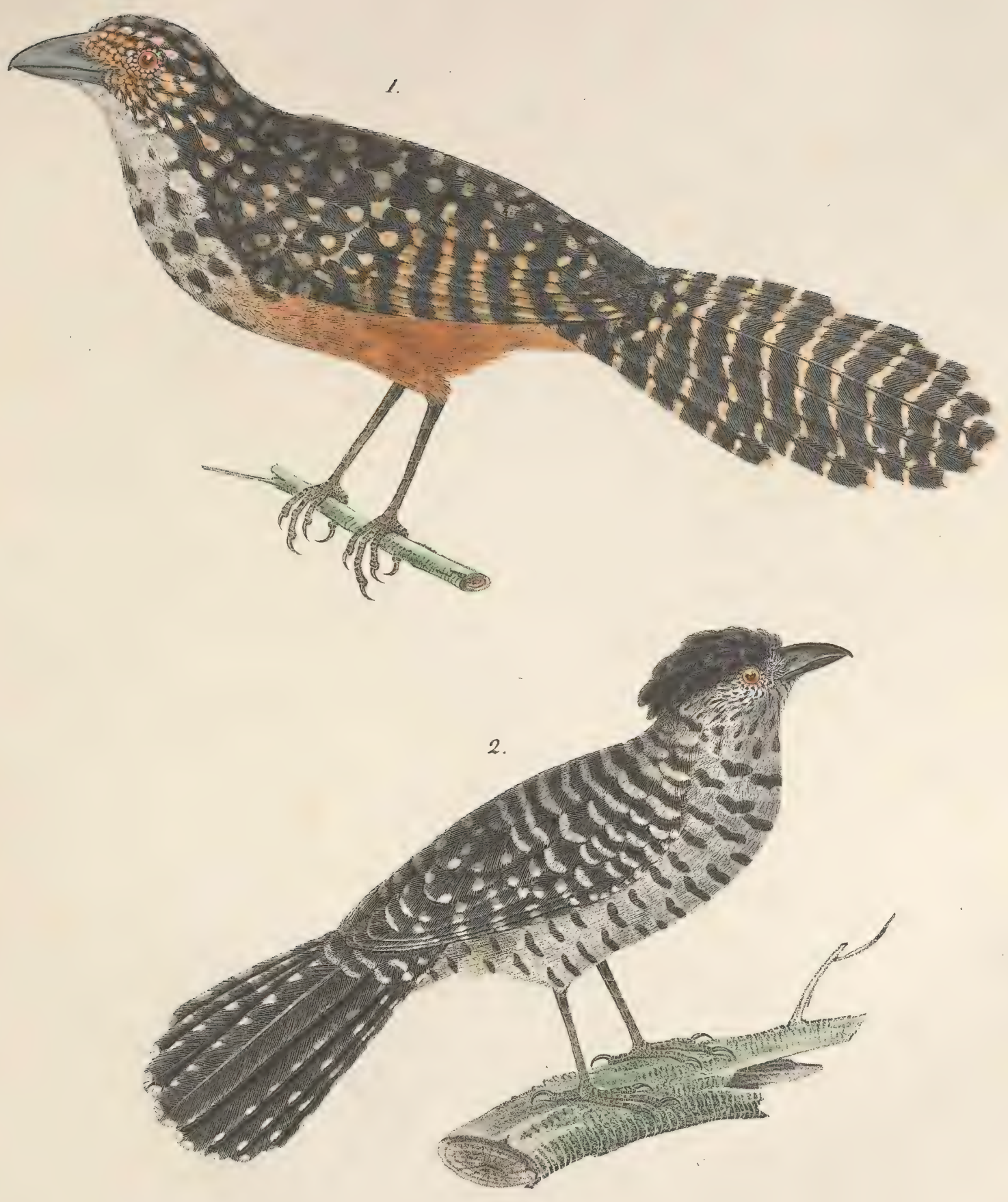

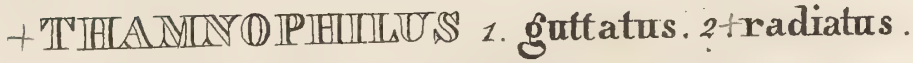

$\mathrm{Tab} . \mathrm{XXXV}$. 


\section{-}



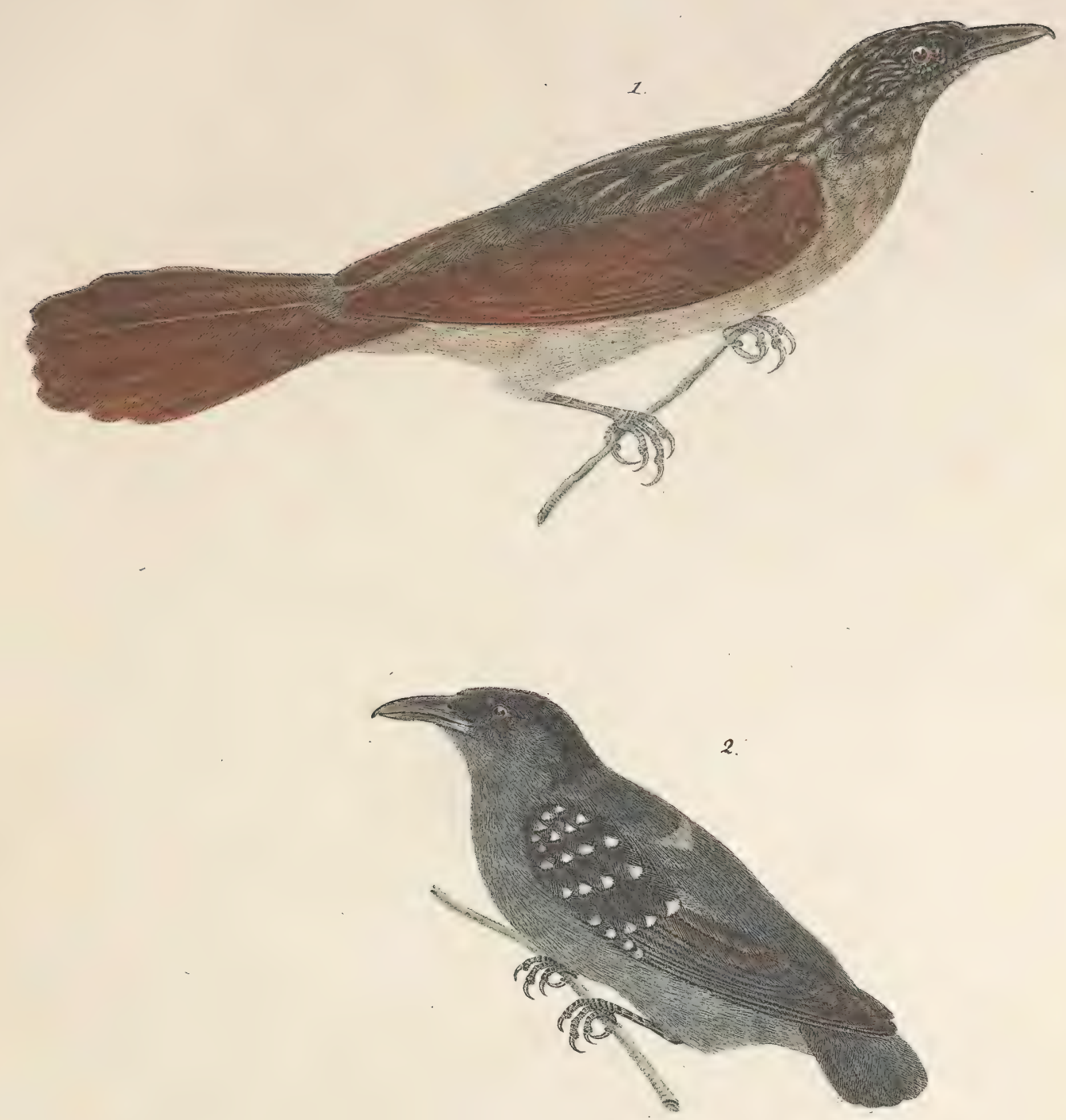

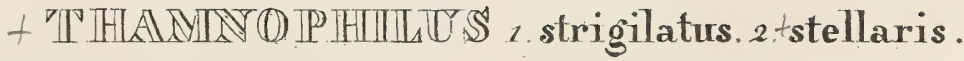

Tab.XXXVI. 



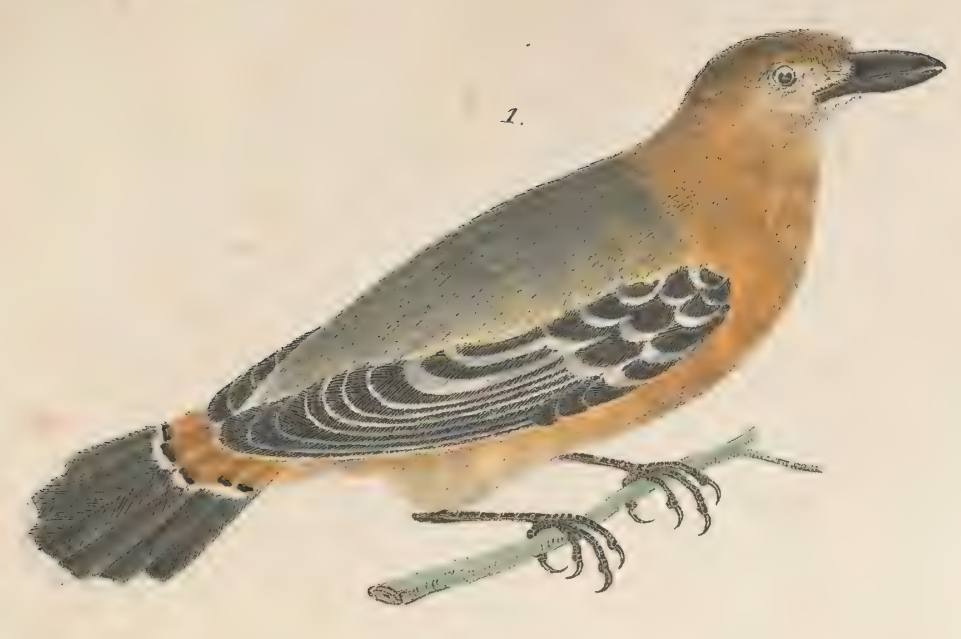

2.

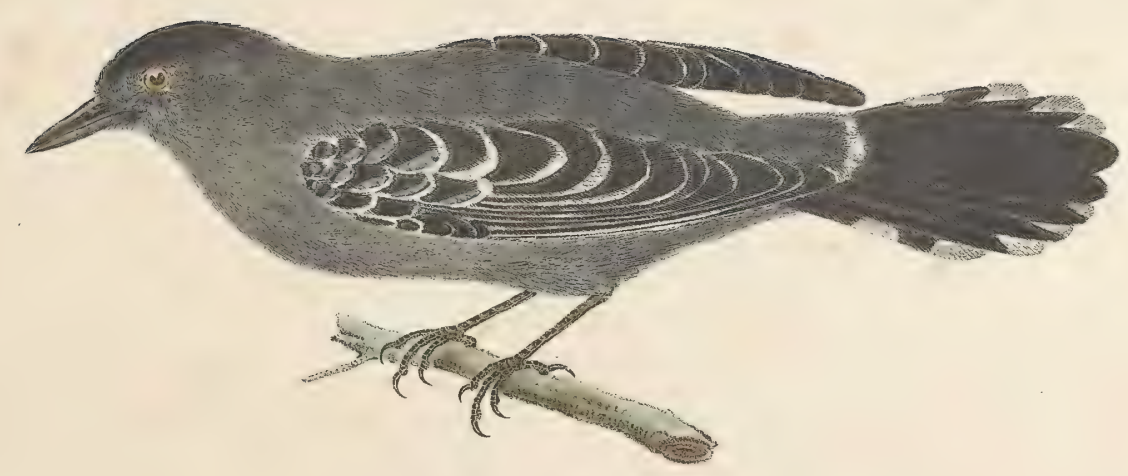

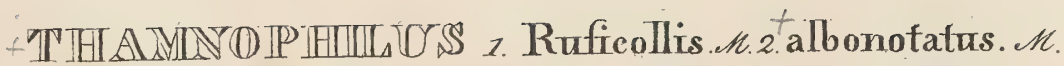

Tab.XXXVII. 


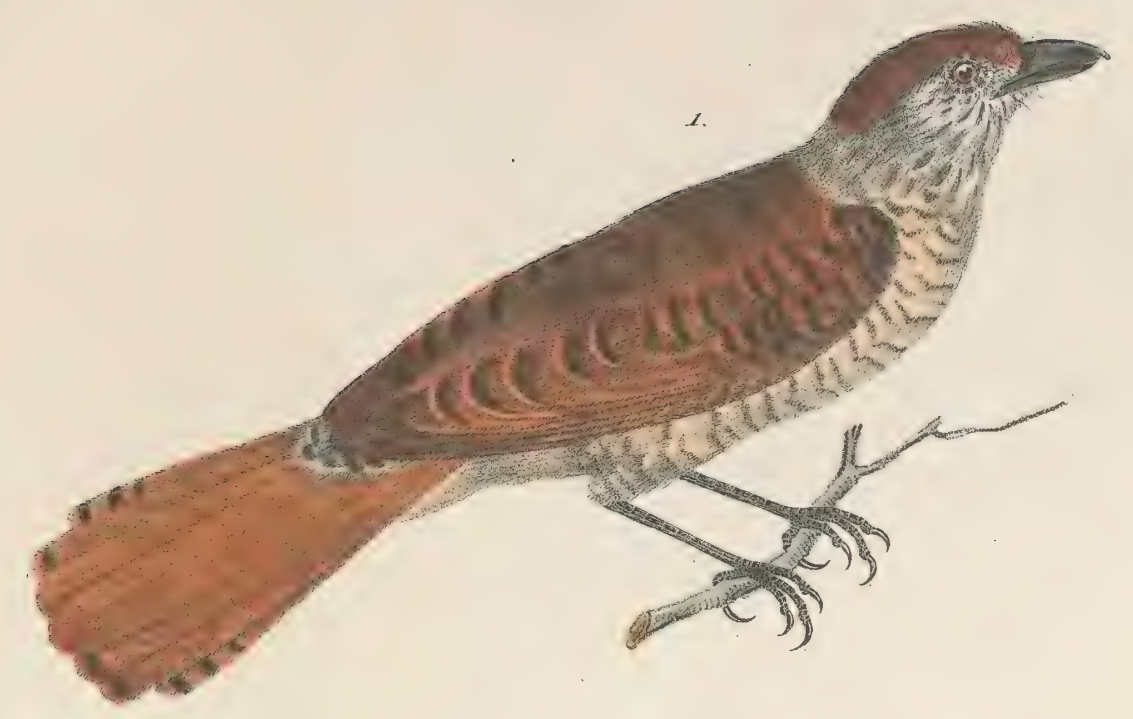

2.

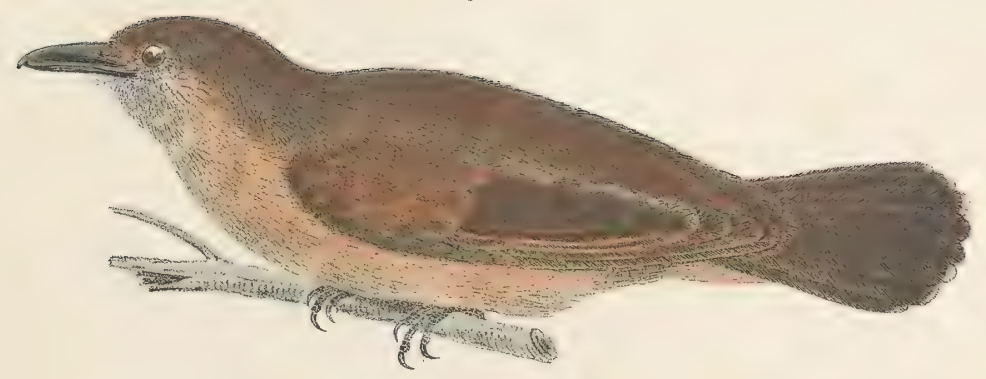

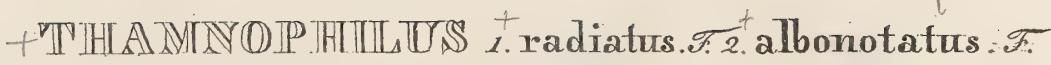

Tab.XXXVIII. 


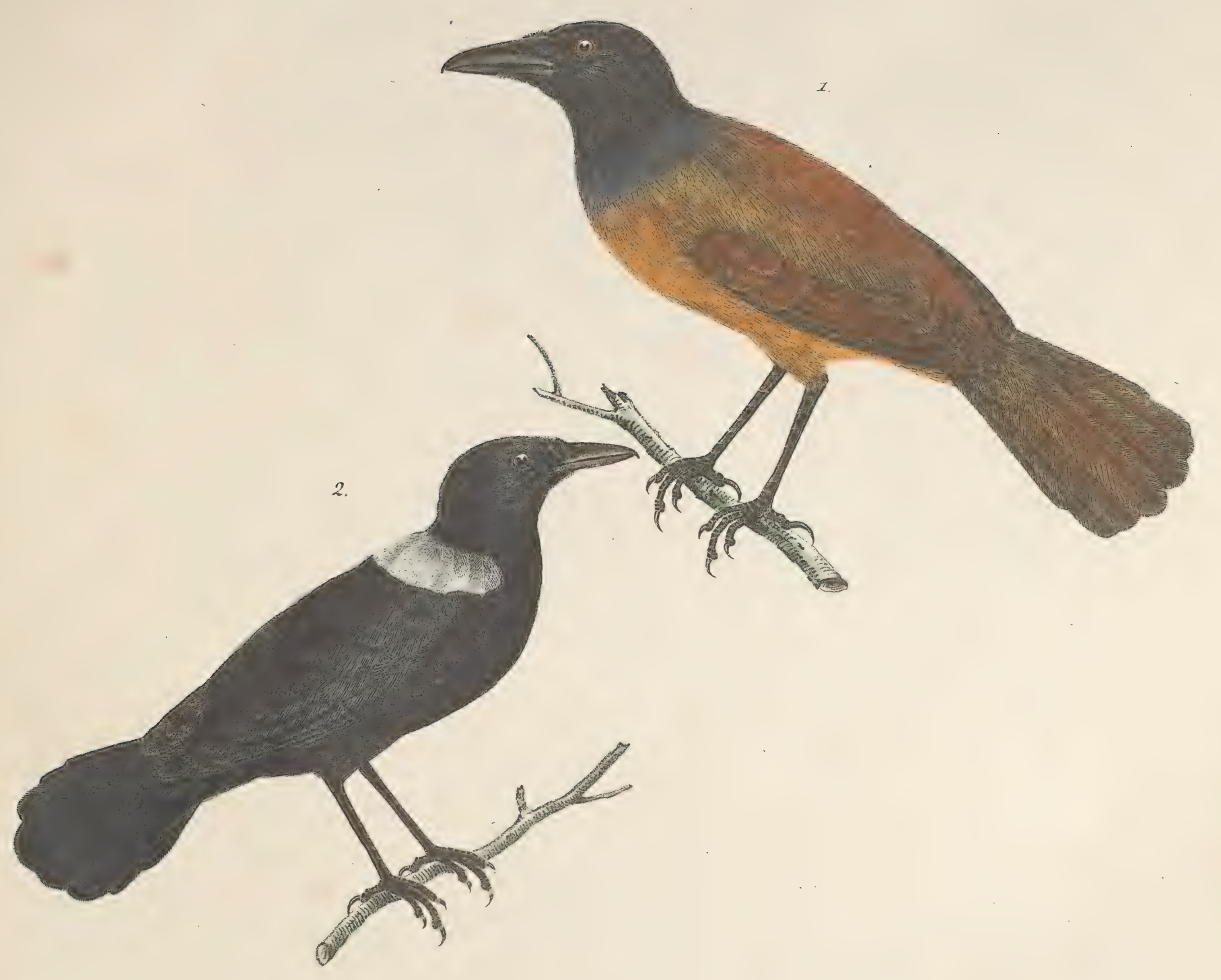

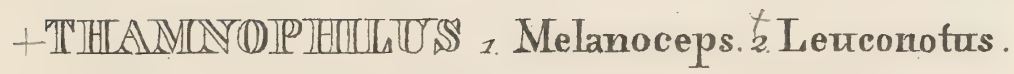

Tab.XXXIX 


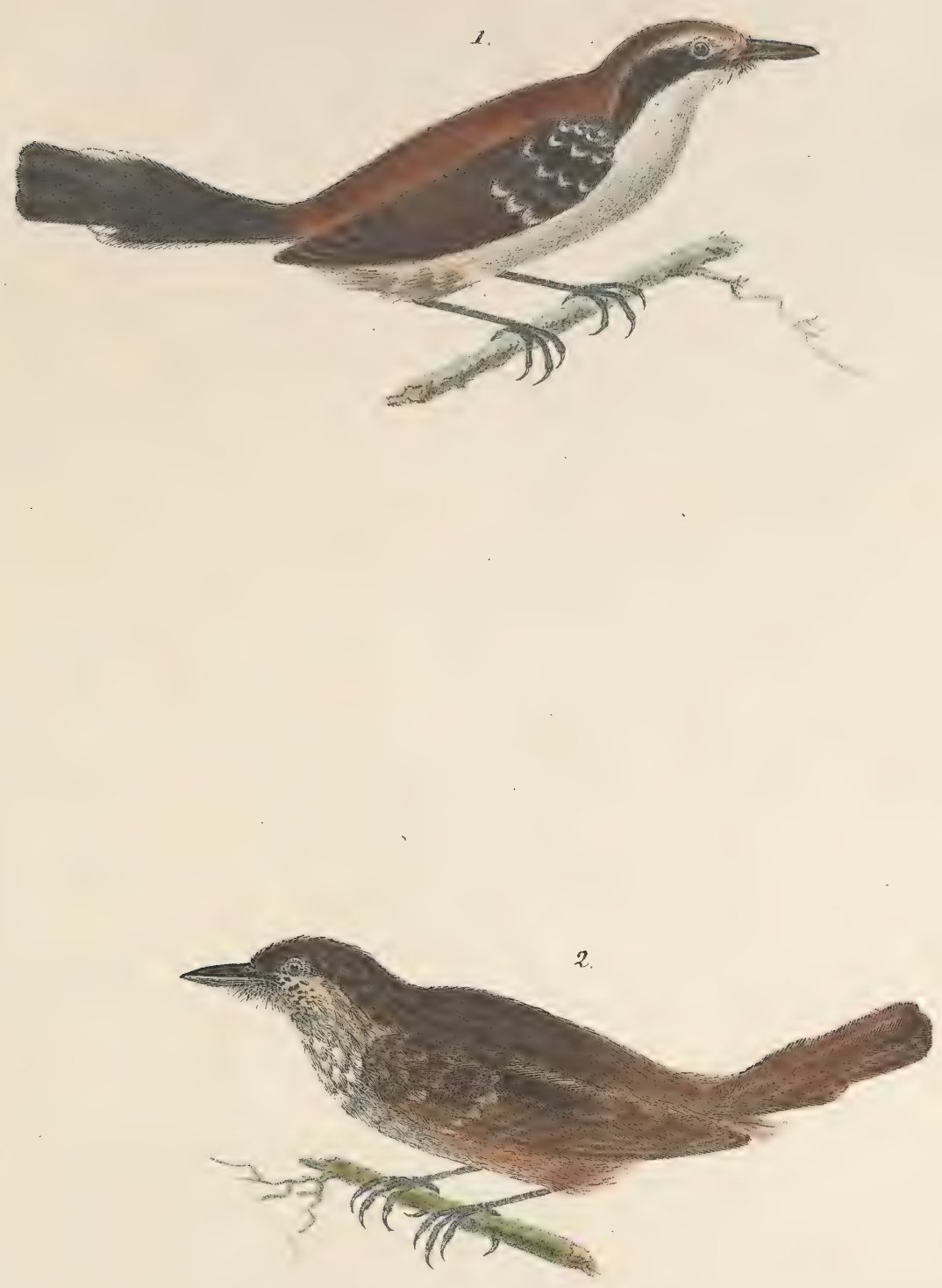

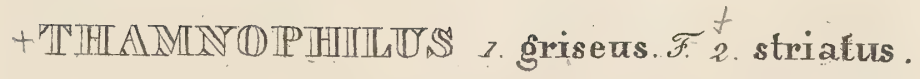

Tab.XT. 


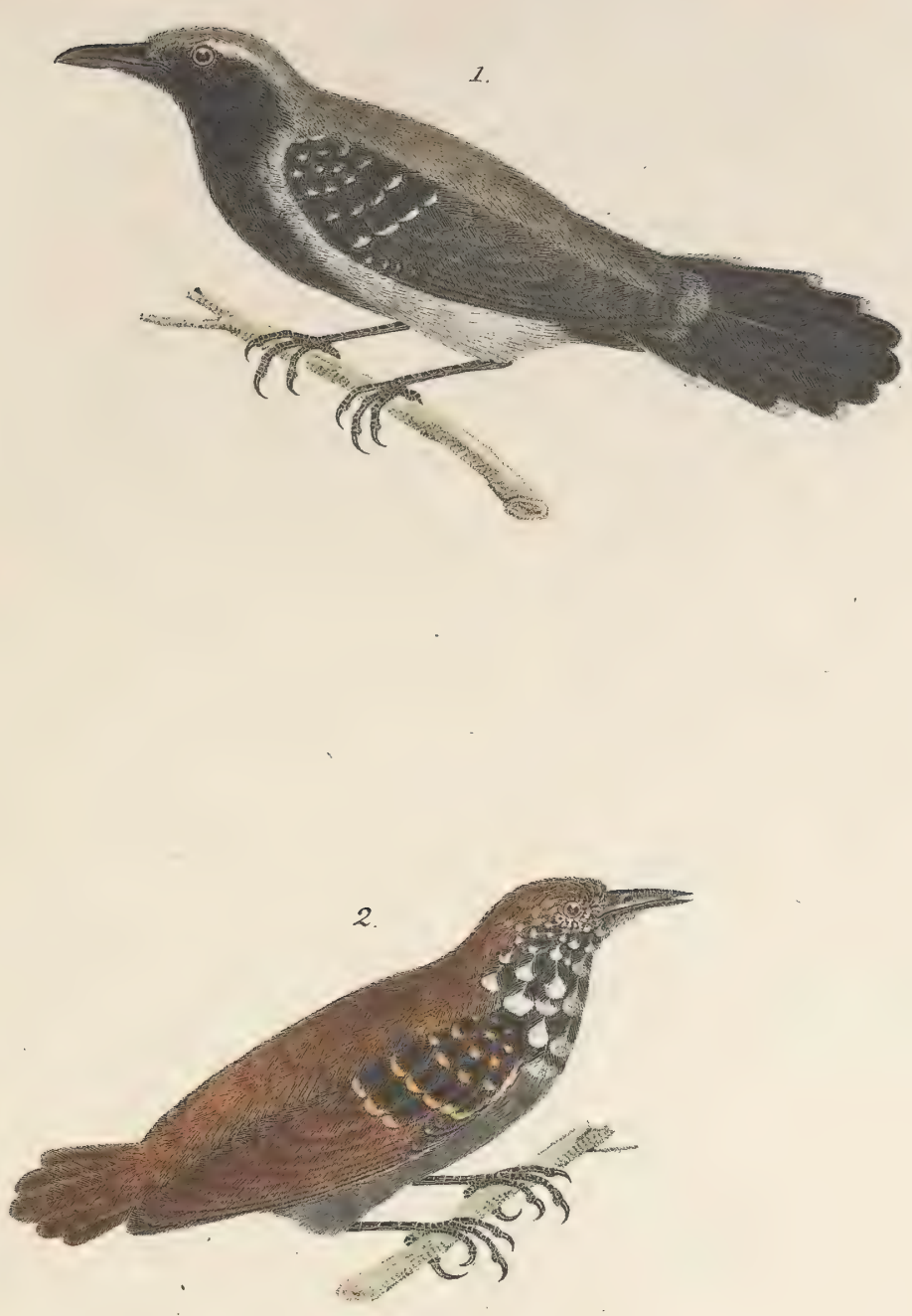

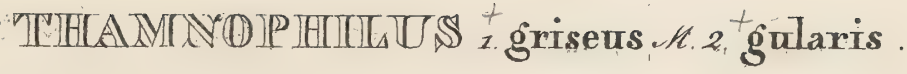

Tab.XLI. 


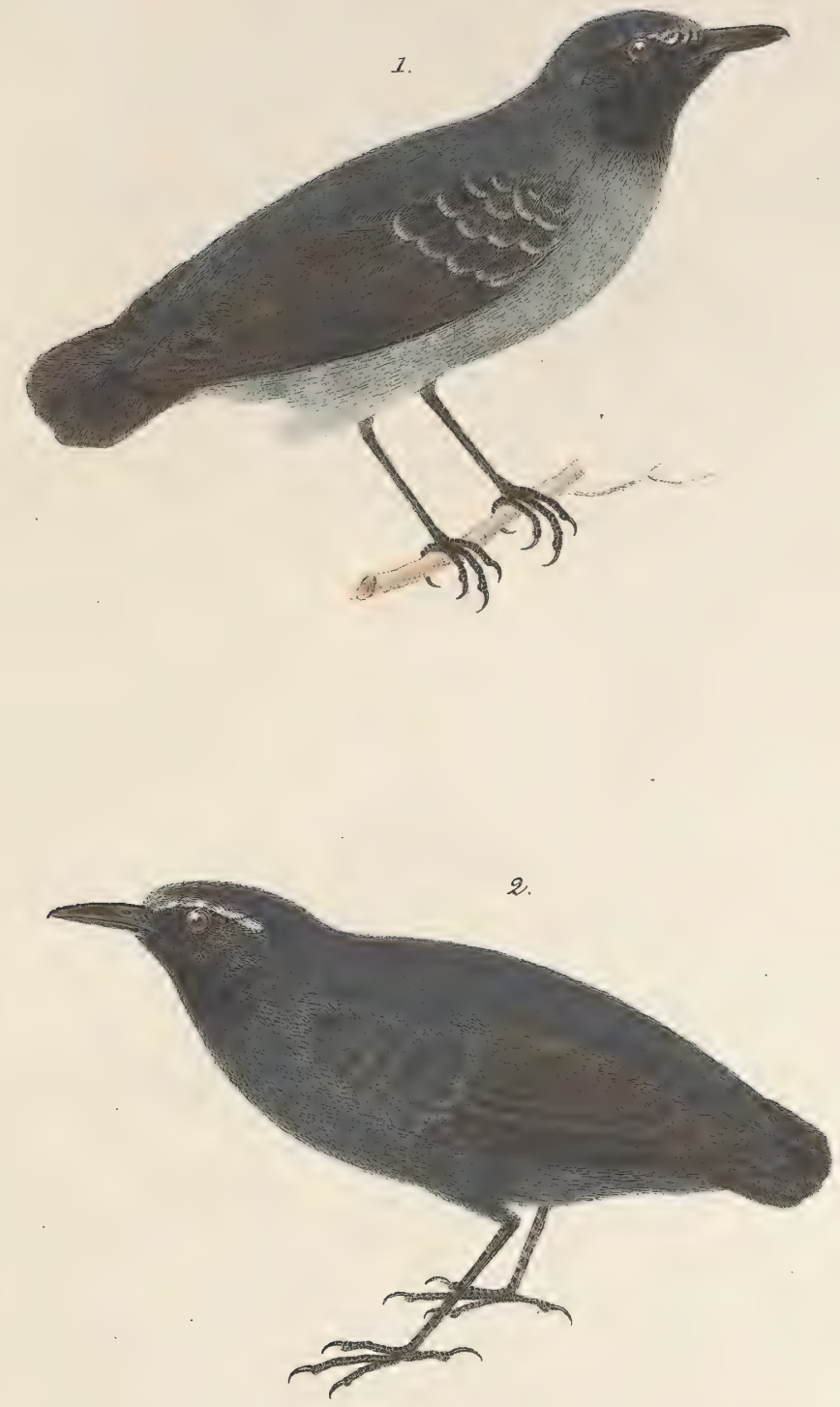

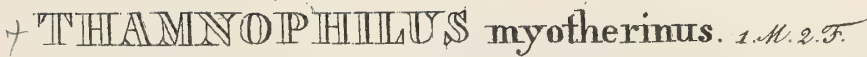

Tab.XLII. 
$c$ 

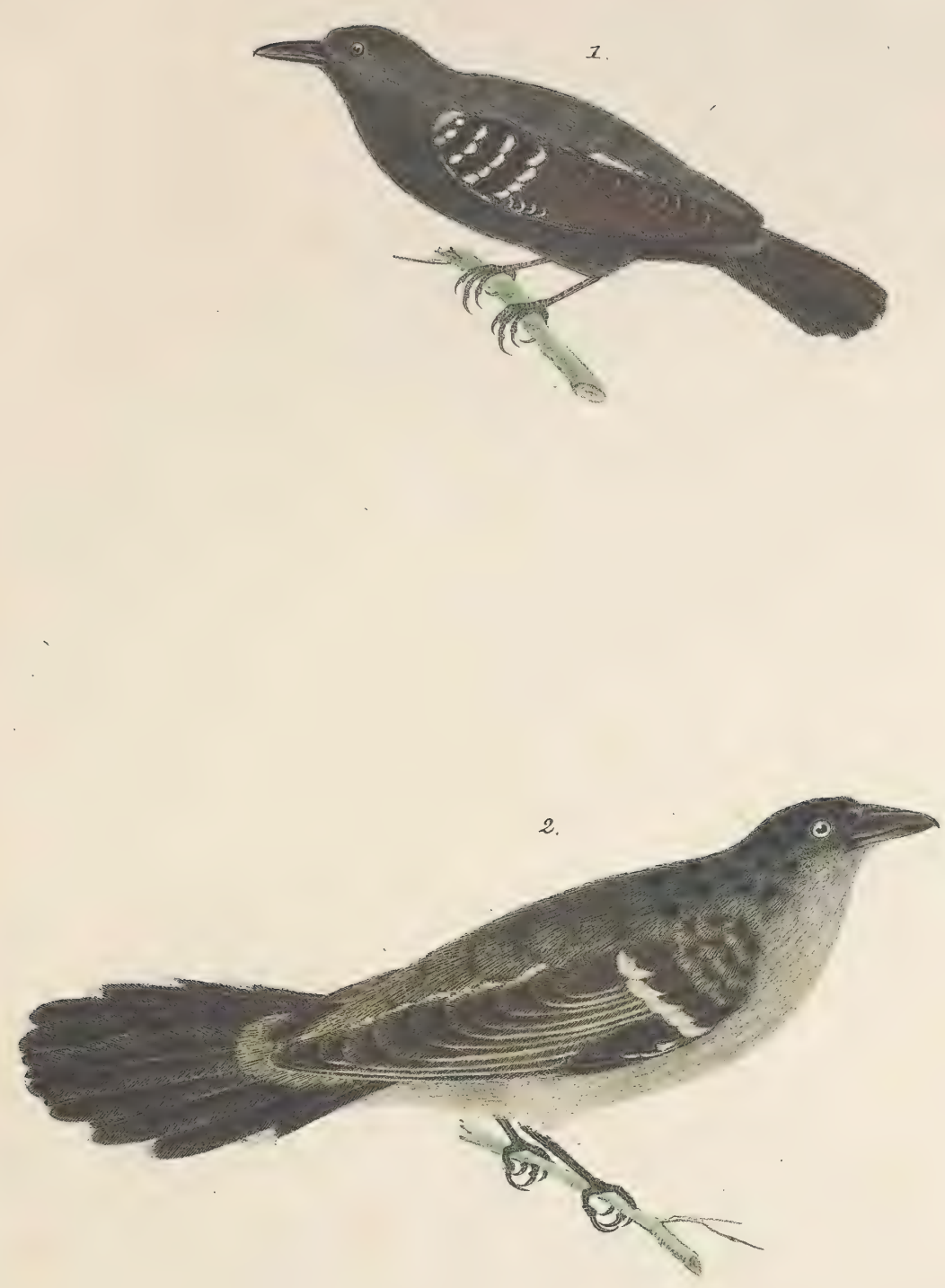

2.

+. TITHAMDNOPIHIIIUSS Melanogaster. t PACCHIYR HIINACTHUS variegatus.

Tab.XLIII . 


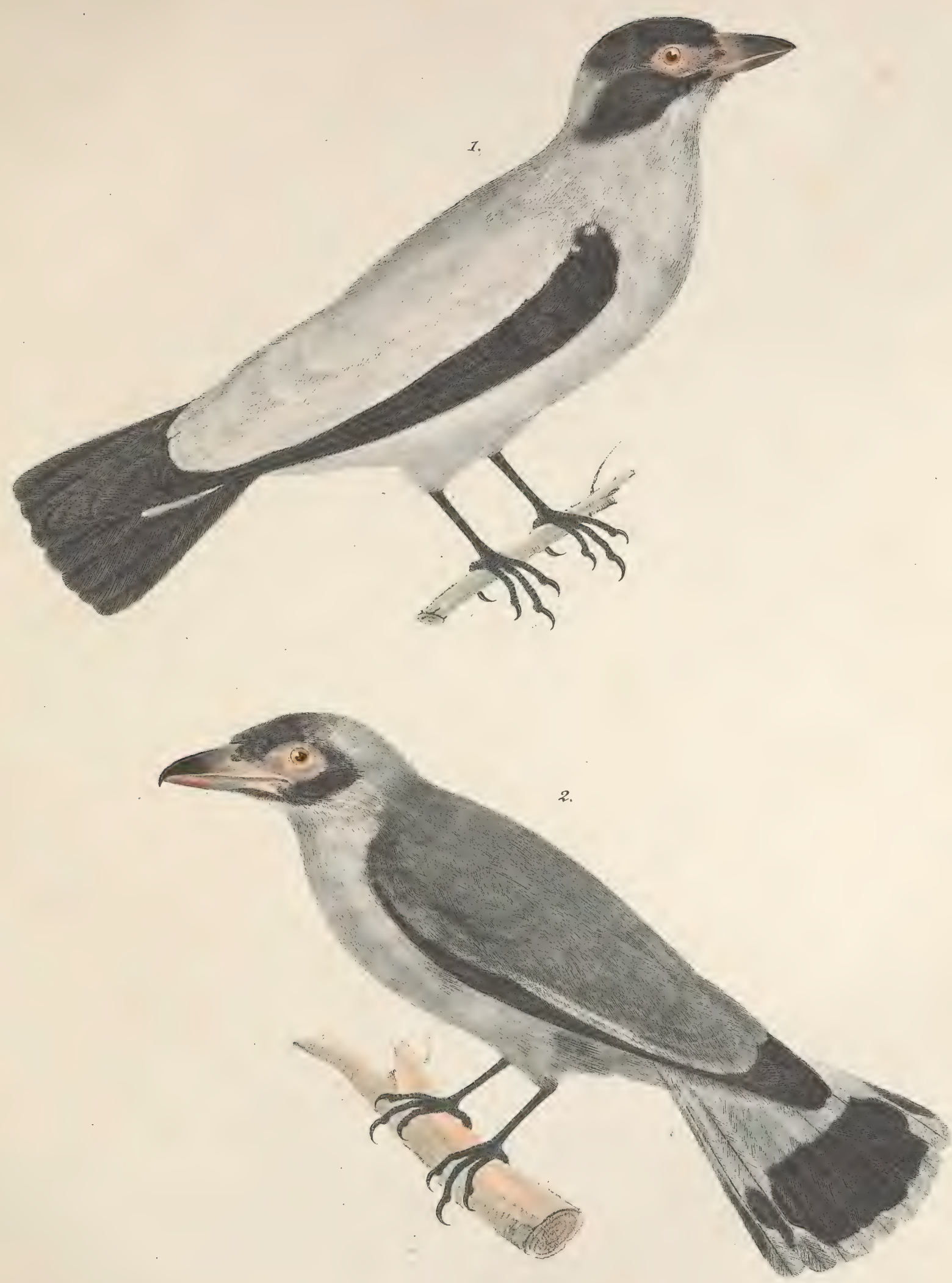

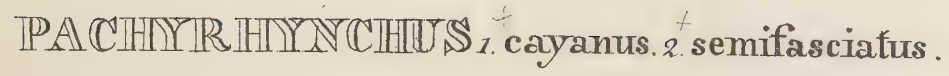

Tab.XLIV. 


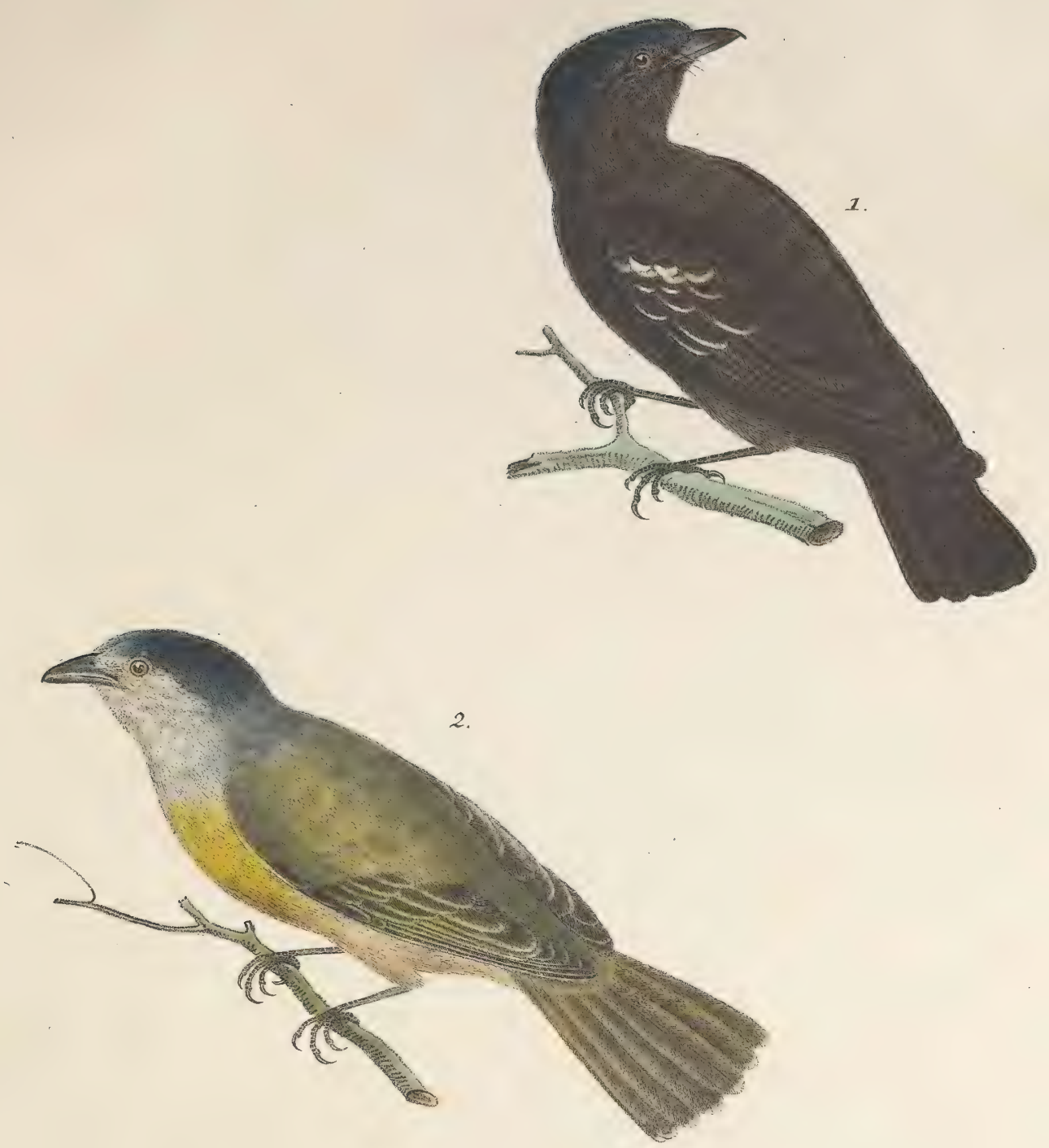

$\mathbb{P}$ ACUHYRTHYNCHUS,

Tab. XLV. 



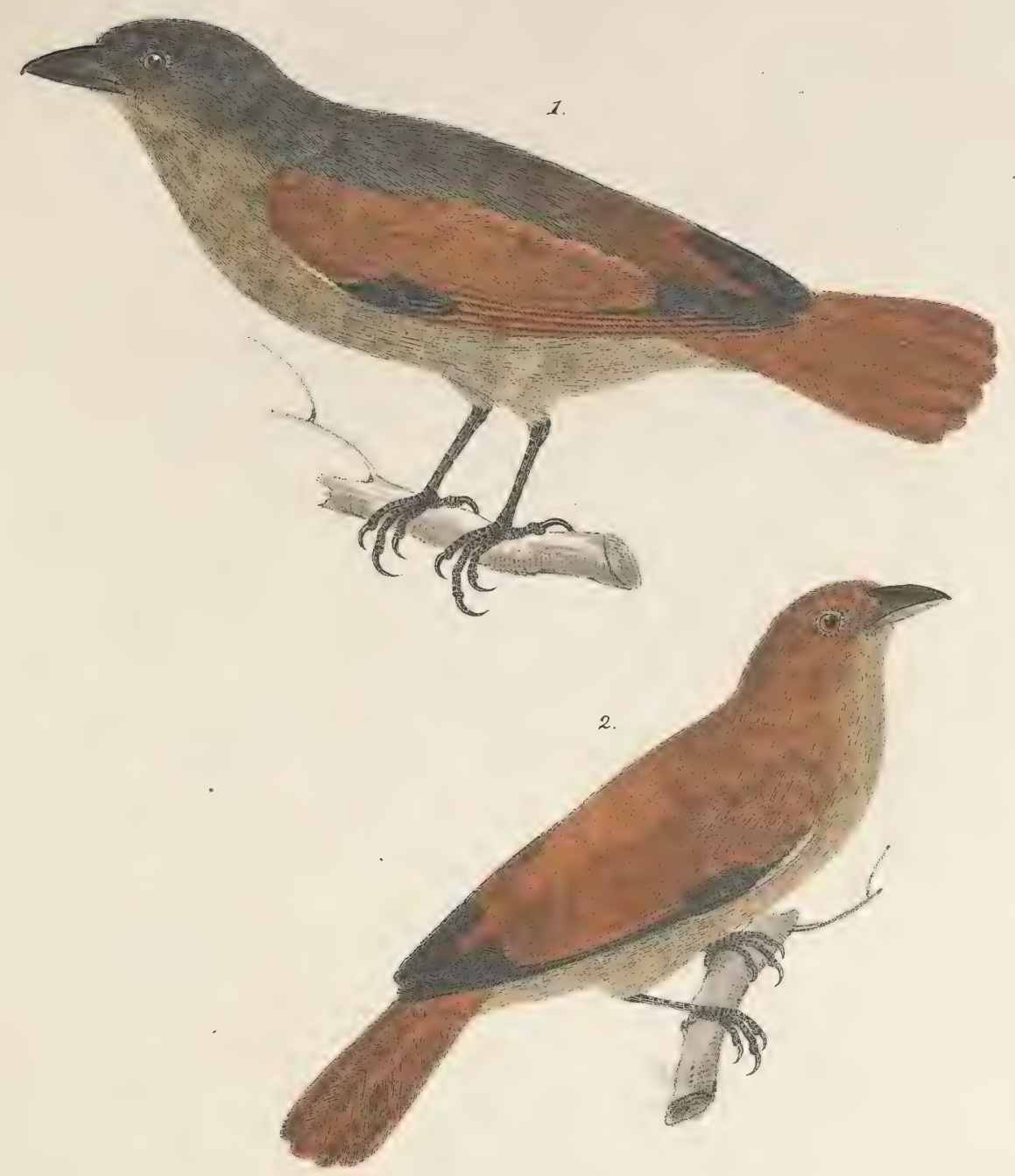

+ PACTHYRRTHYNCTHUS, cinerascens. ${ }_{2}$. rufescens .

Tab.XI,VI . 


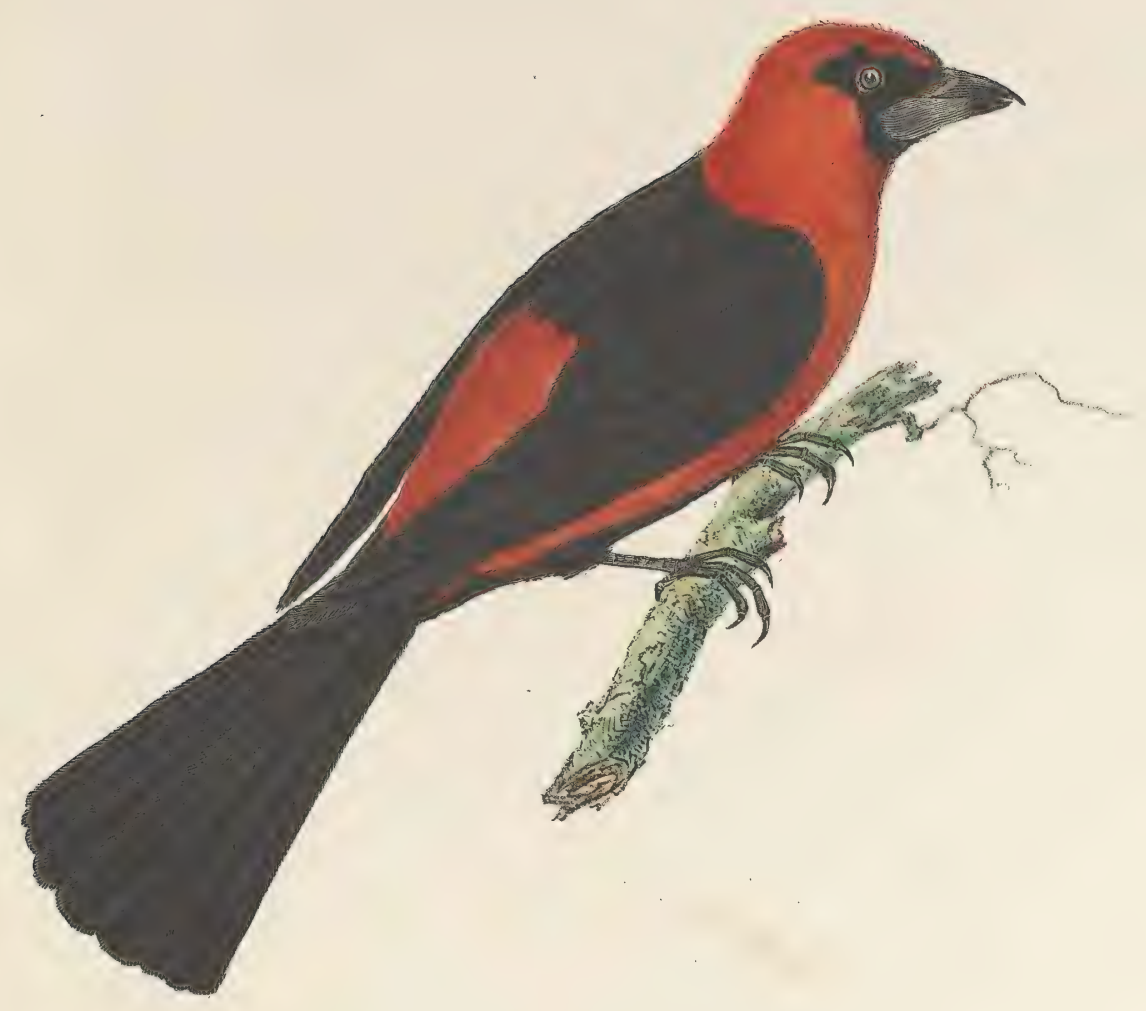

+ $\mathbb{P A N A} \mathbb{N} \mathbb{R}$ Al nigrogularis.."

Tab.XLVII . 


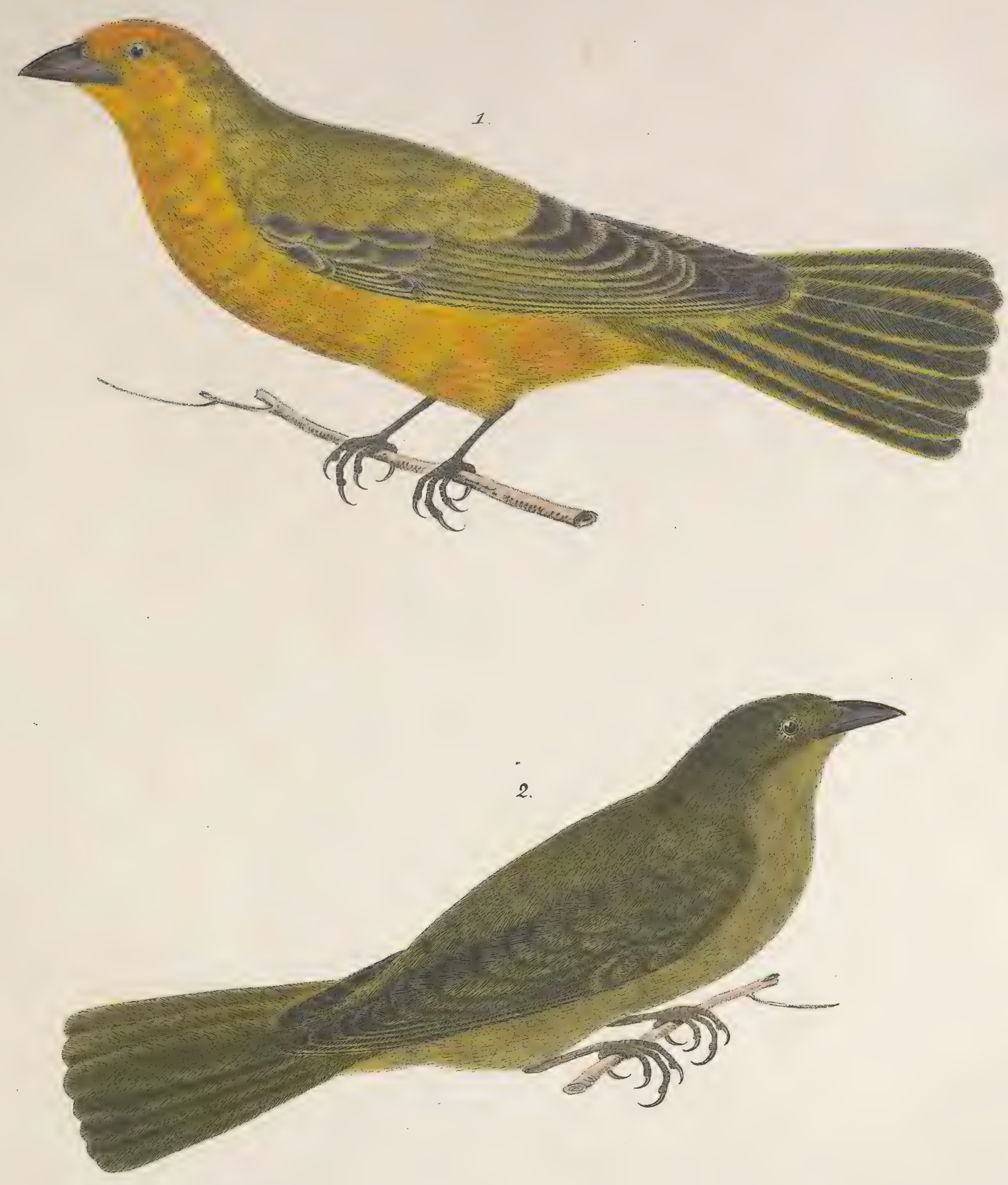

+ IPANA

Tab. XLVIII . 

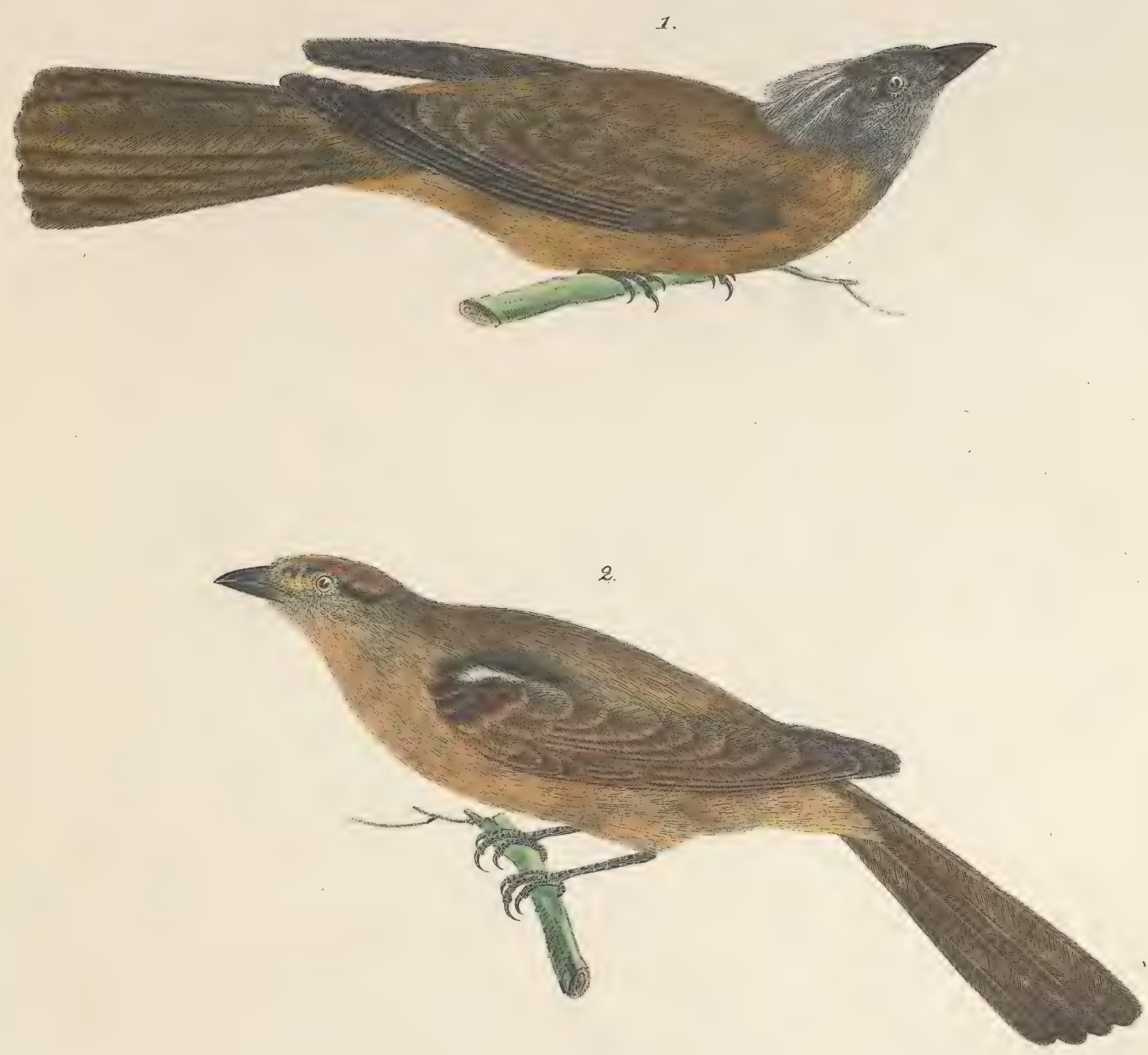

TIANAGR A t. penicillata. ${ }^{+}$brunnea .

Tab. XLIX. 



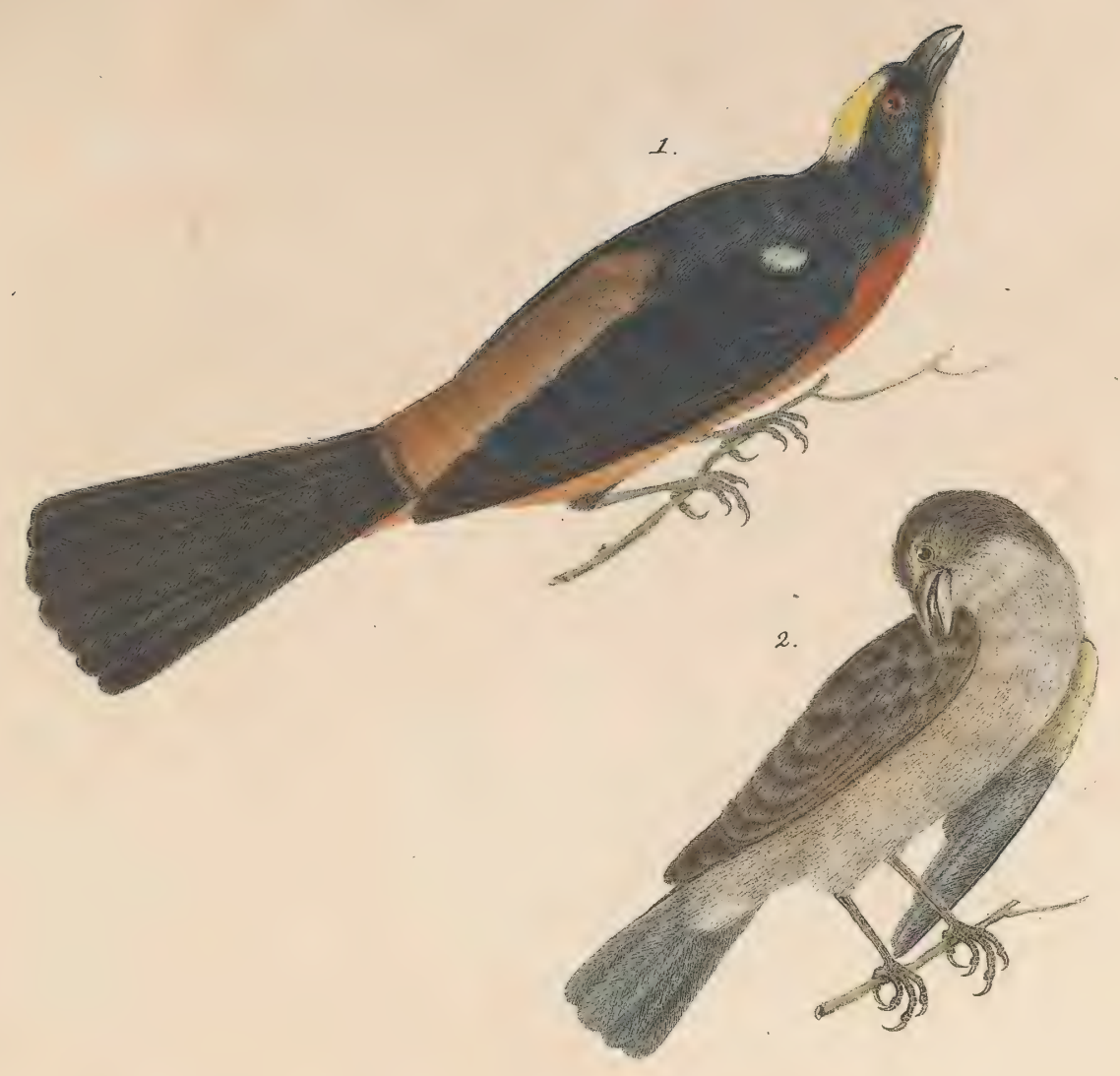

+ ${ }^{\mathbb{I}} \mathbb{A} \mathbb{N} \mathbb{A} \mathbb{G} \mathbb{R}$, . Rufiventer. ${ }^{+}$Aurifrons.

Tab.I . 


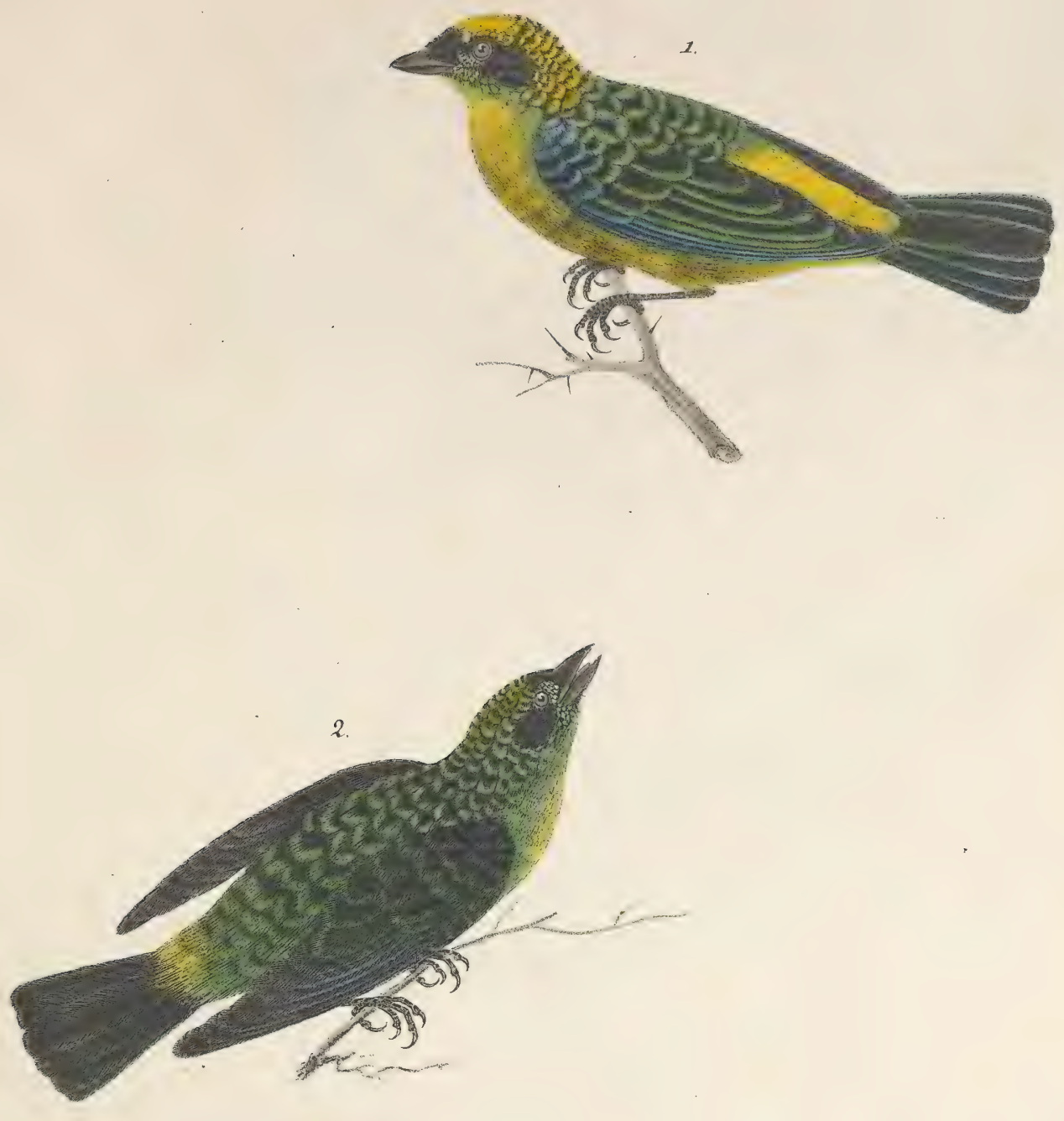

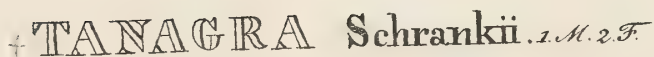

Tab.LI. 


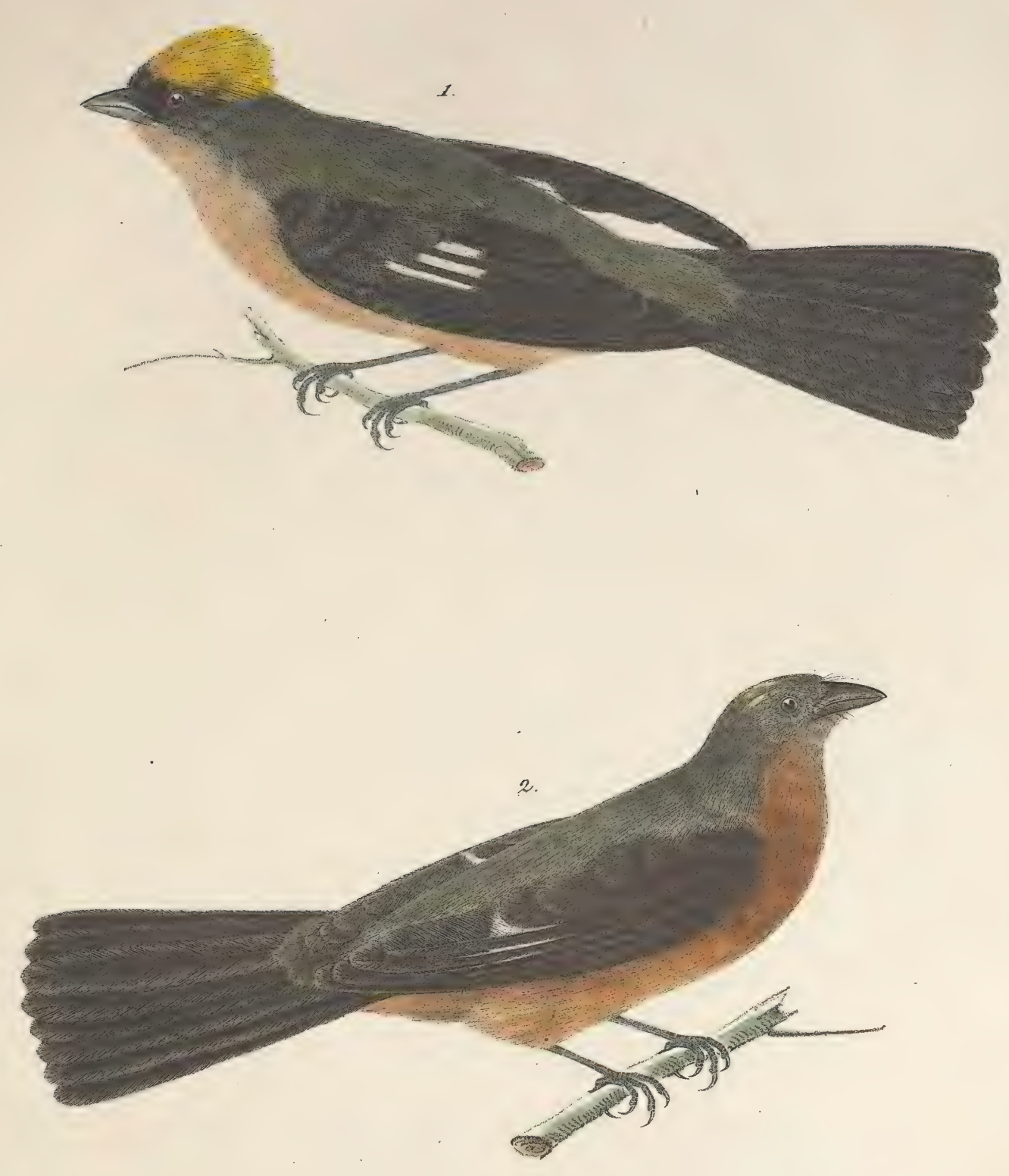

- TANAGRA Auricapilla.1.2.\%

Tab:LII . 



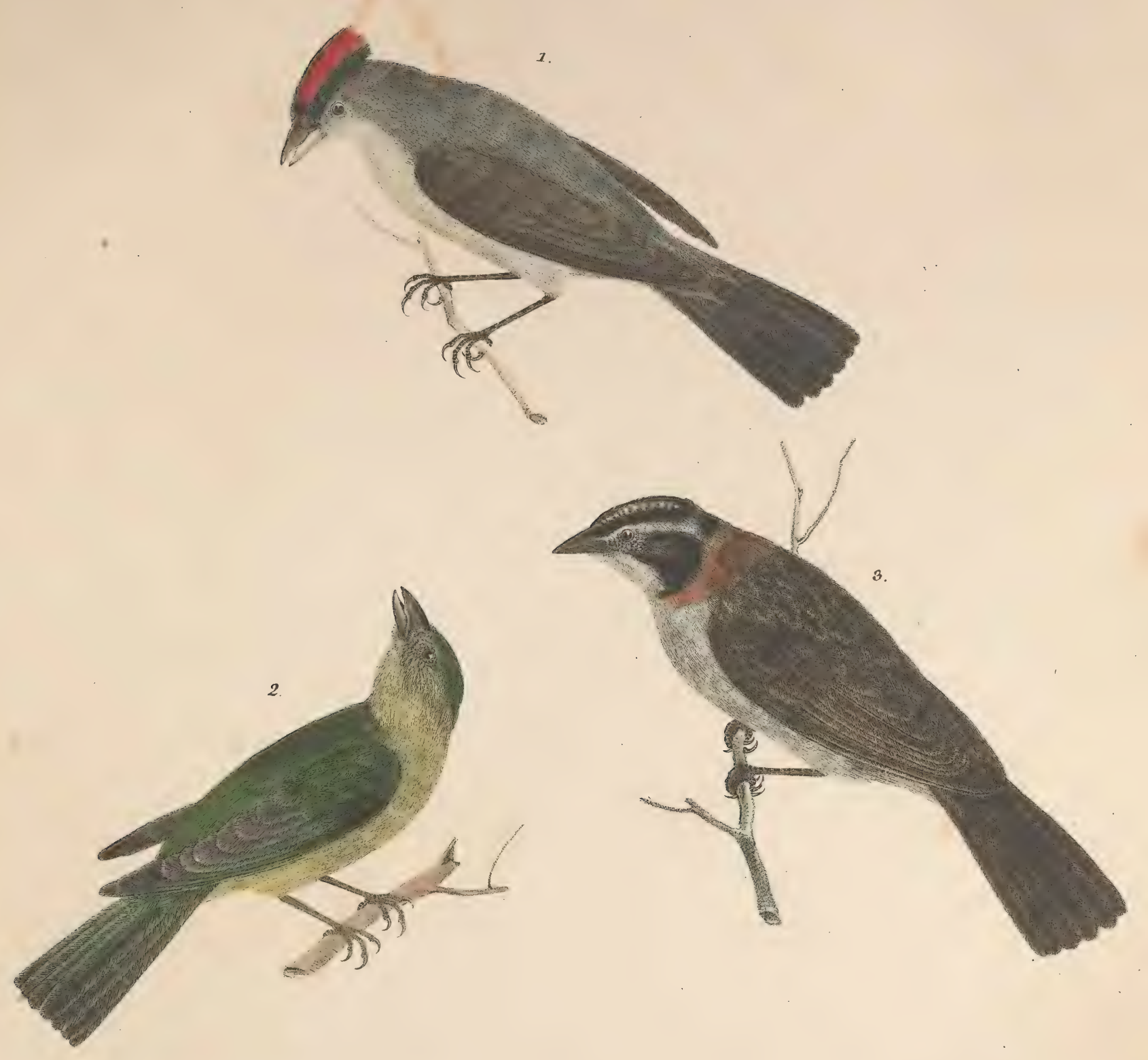

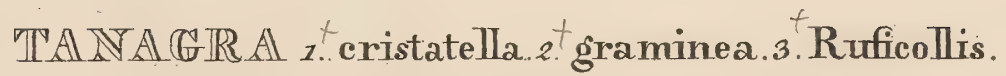

Tab.LIII . 

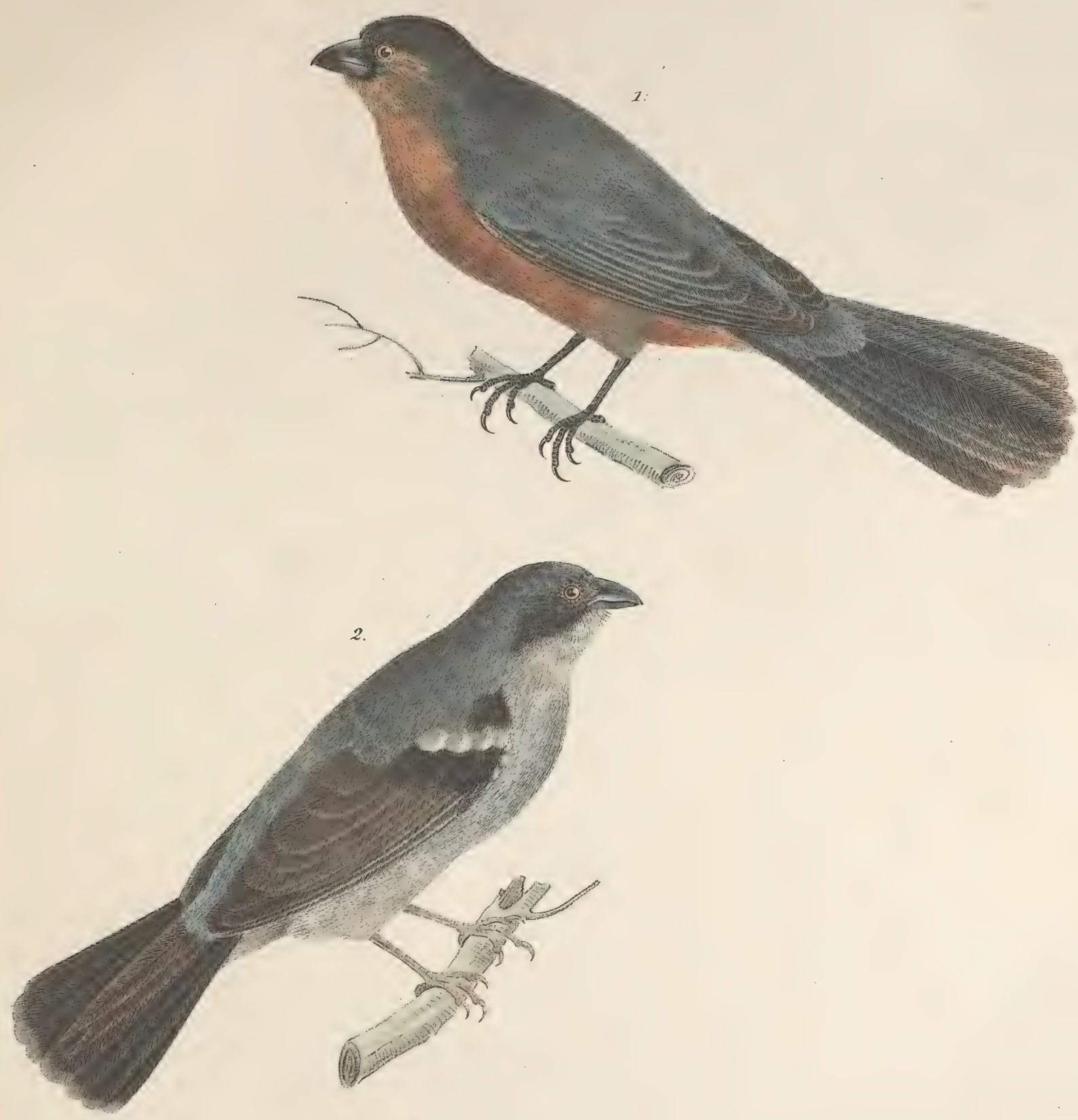

TANVAORA ${ }_{1}$ teapistrata $^{+}$axillaris .

T2b : LIV. 


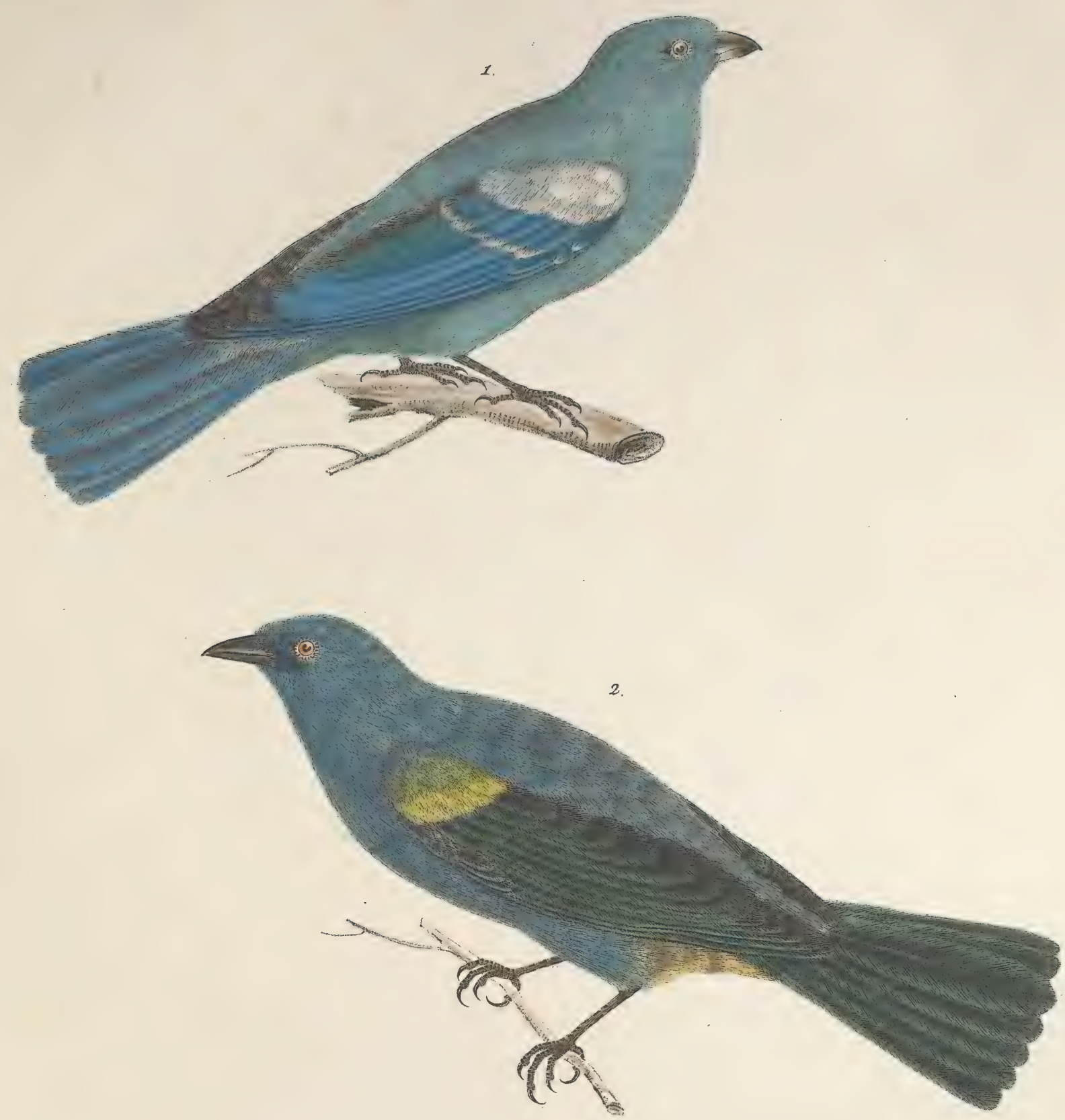

+ TANAGR A z.coelestis. . A Archiepiseopus.

Tab.LV. 

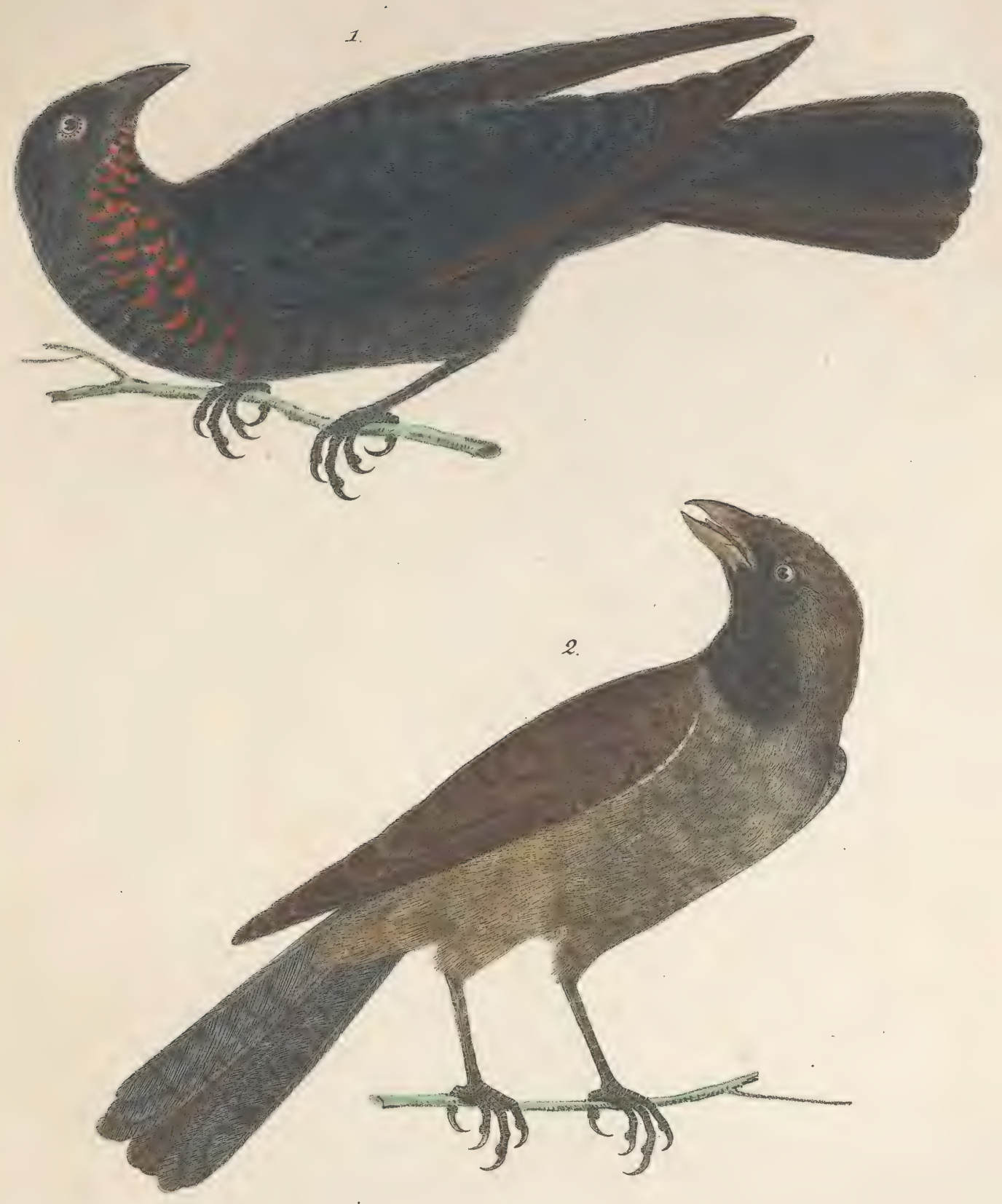

TIANAGRA rubrigularis . F. 2. Atricollis. .

Tab. LVI. 



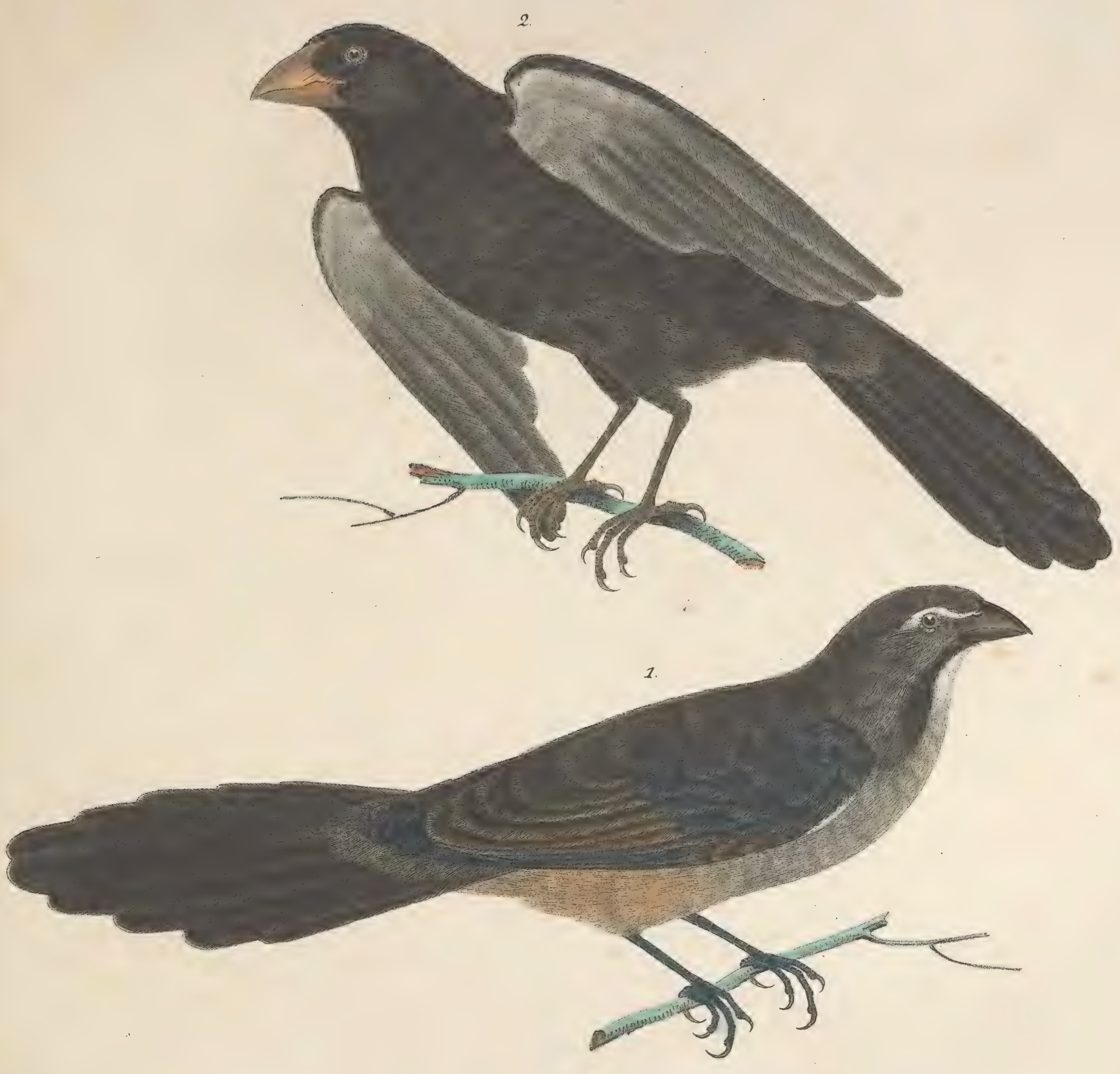

$+{ }^{\top} \mathbb{A} \mathbb{N}$ (G) $\mathbb{R}$ A 1.superciliaris. 2.psittacina .

Tab. LVII . 


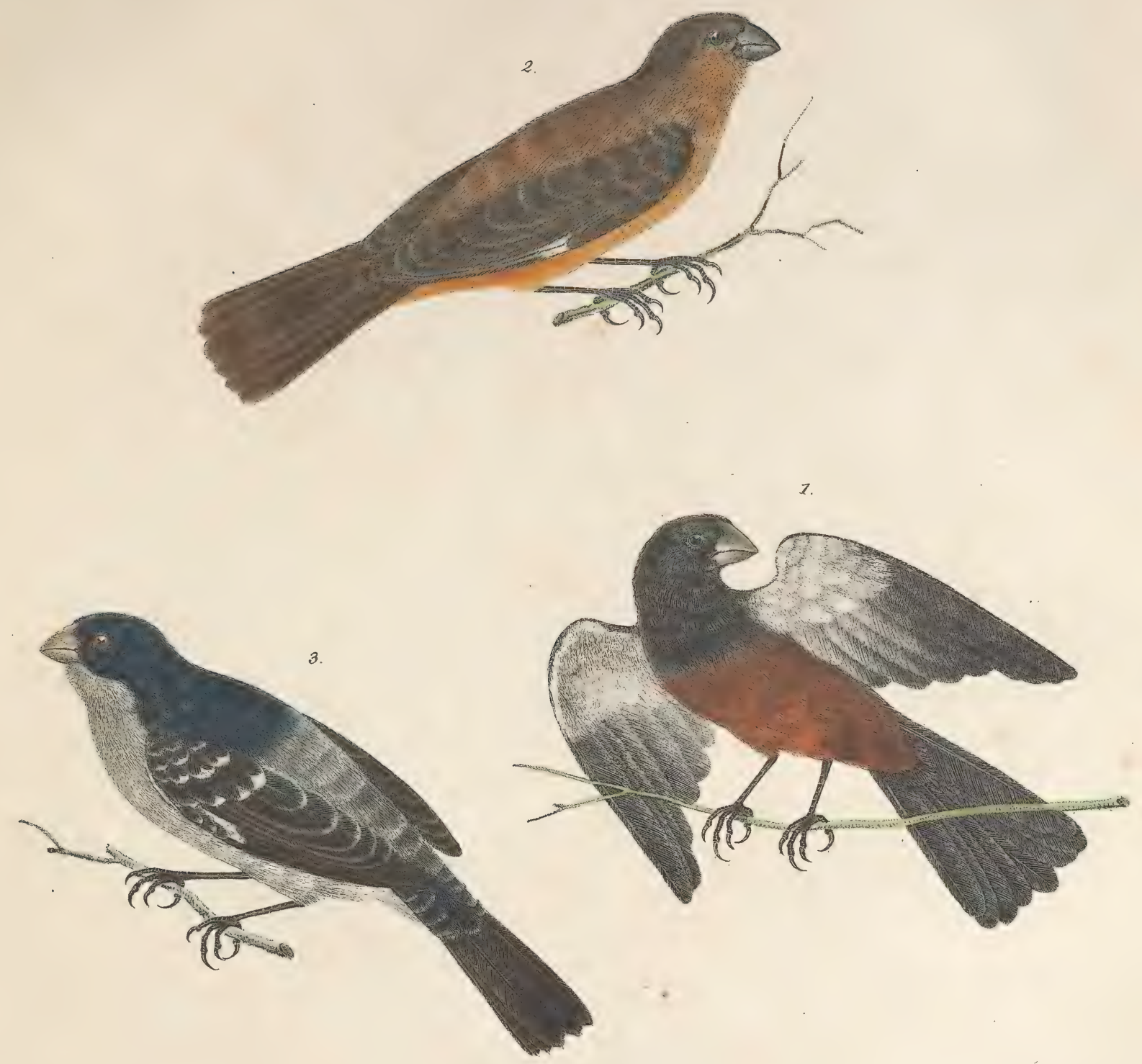

+ IIOXIA nasuta.z. 2.F. Ieucopterygia.

Tab. IAVIII . 

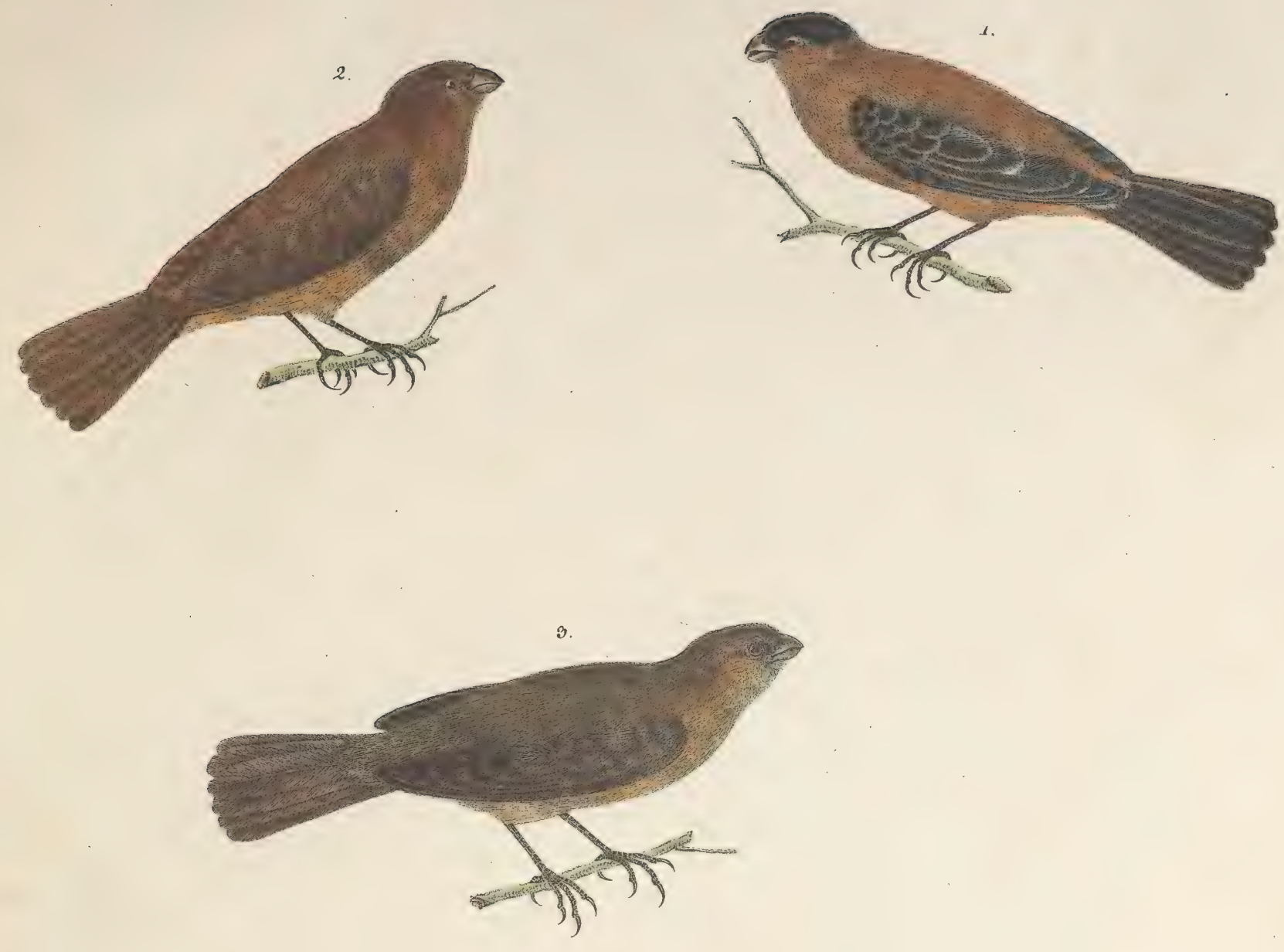

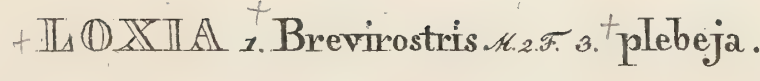

Tab.IIX . 


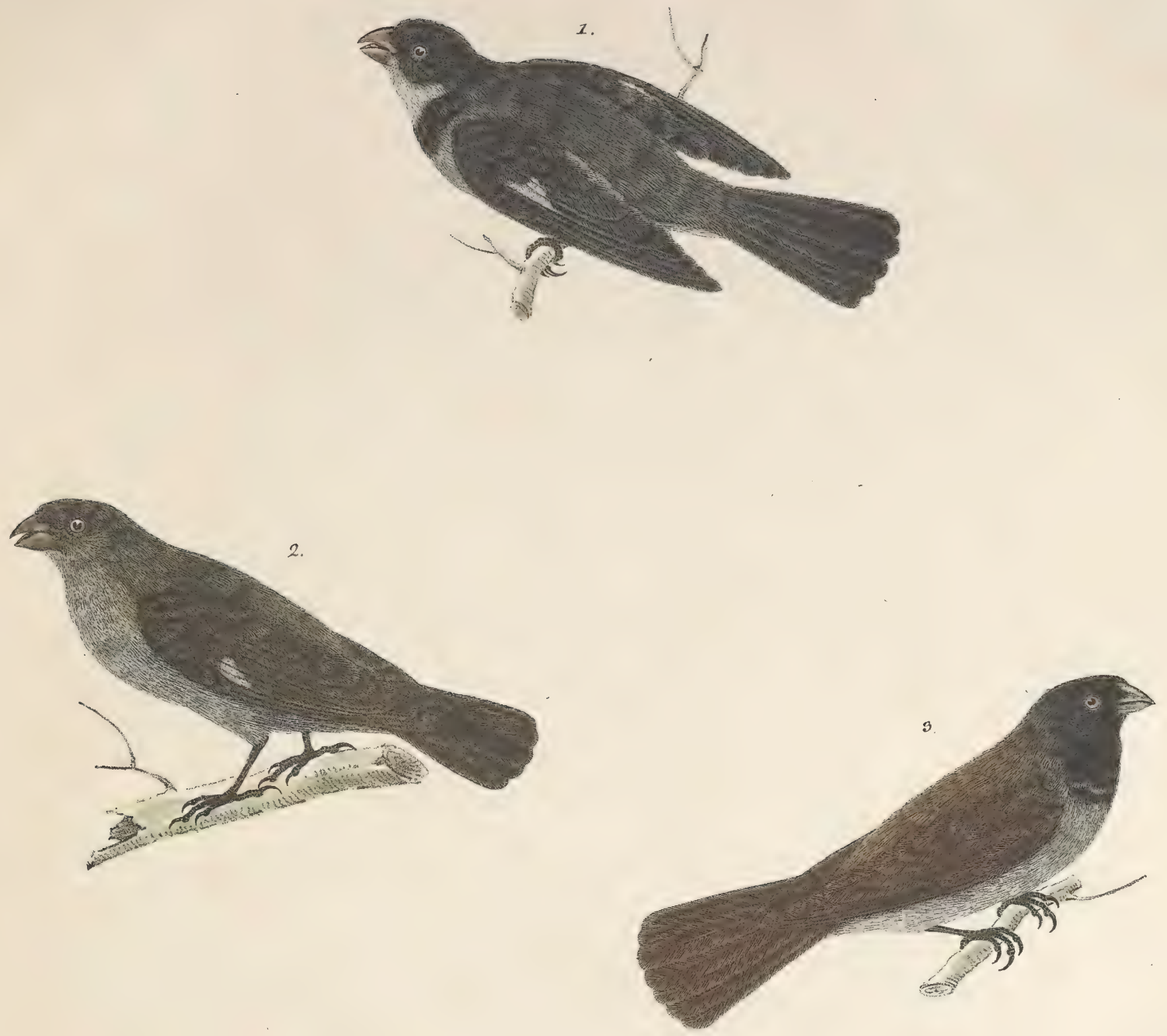

ISOXIIA r albogularis. N.2.F z. ignobilis .

Tab.LX. 


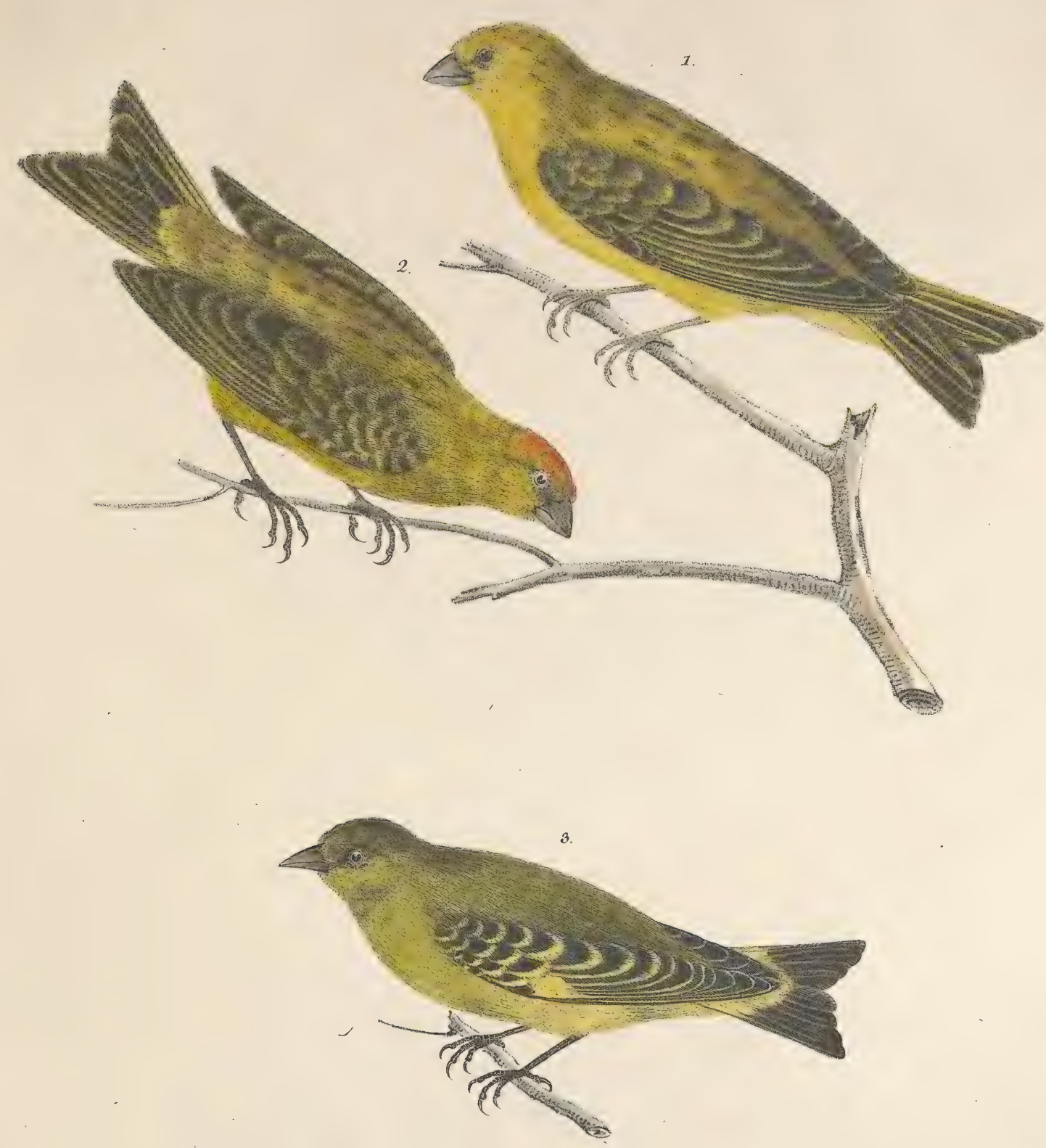

+ IF $\mathbb{R} \mathbb{R} \mathbb{V}(\mathbb{G}) \mathbb{H}_{1} \mathbb{I}_{4} \mathbb{A}$ brasiliensis.7.2.K. 3. campestris.

Tab.LXI. 



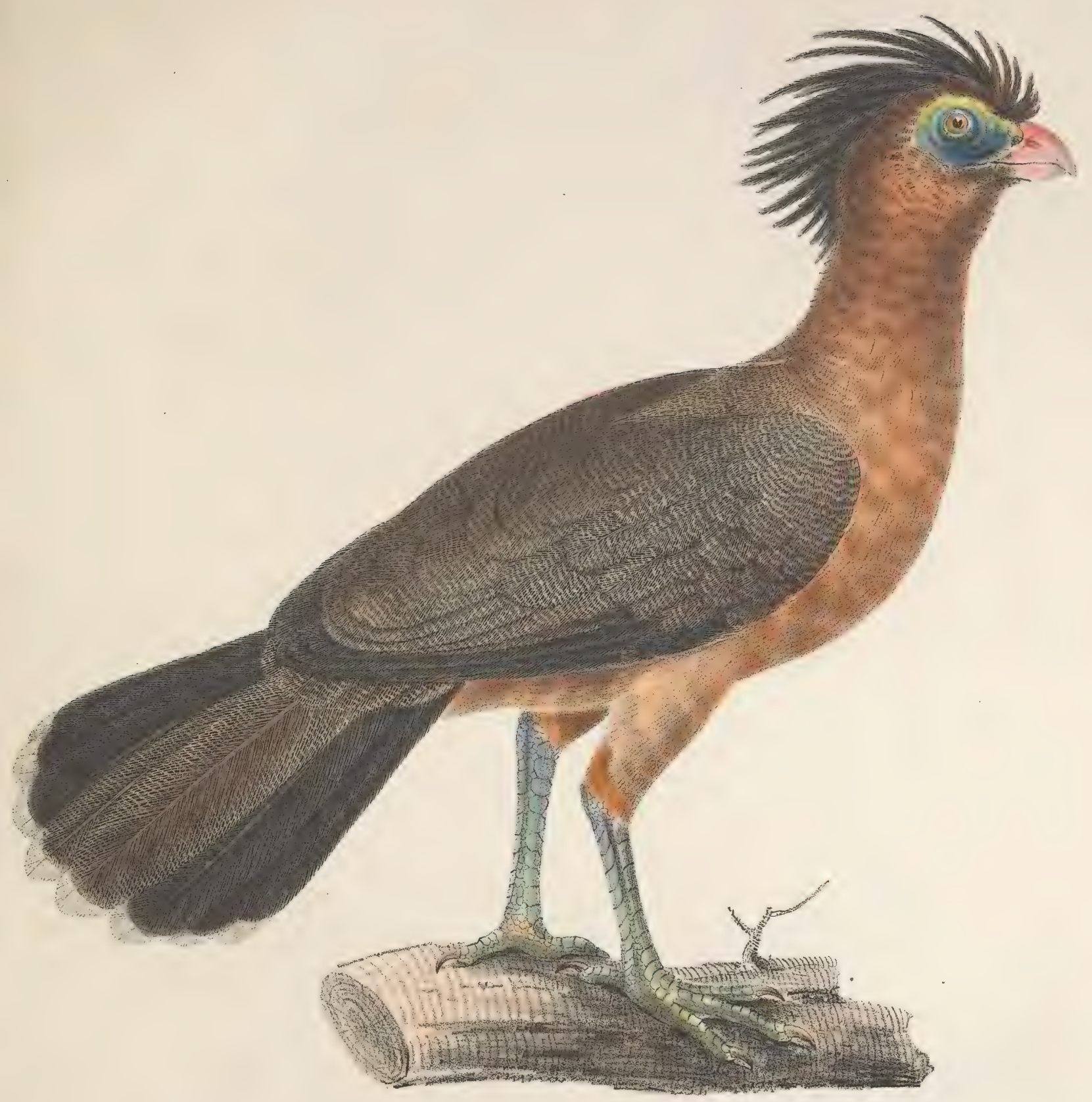

$\because \mathbb{C} \mathbb{R} A \mathbb{X}$ Urumutum .

Tab.LXII. 



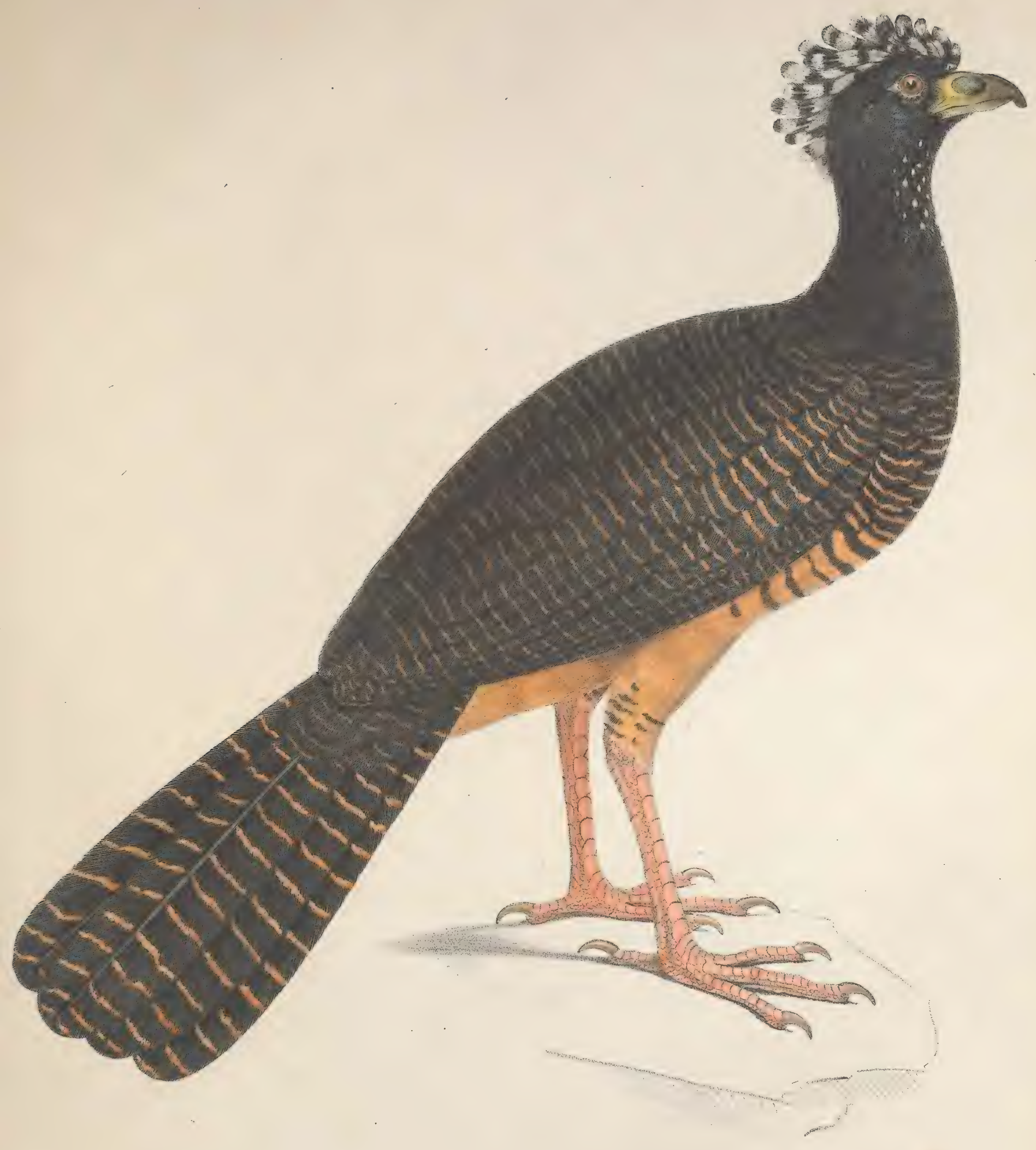

$+\mathbb{C R} A \mathbb{X}$ fasciolata.

Tab.LXII $\stackrel{a}{-}$ 



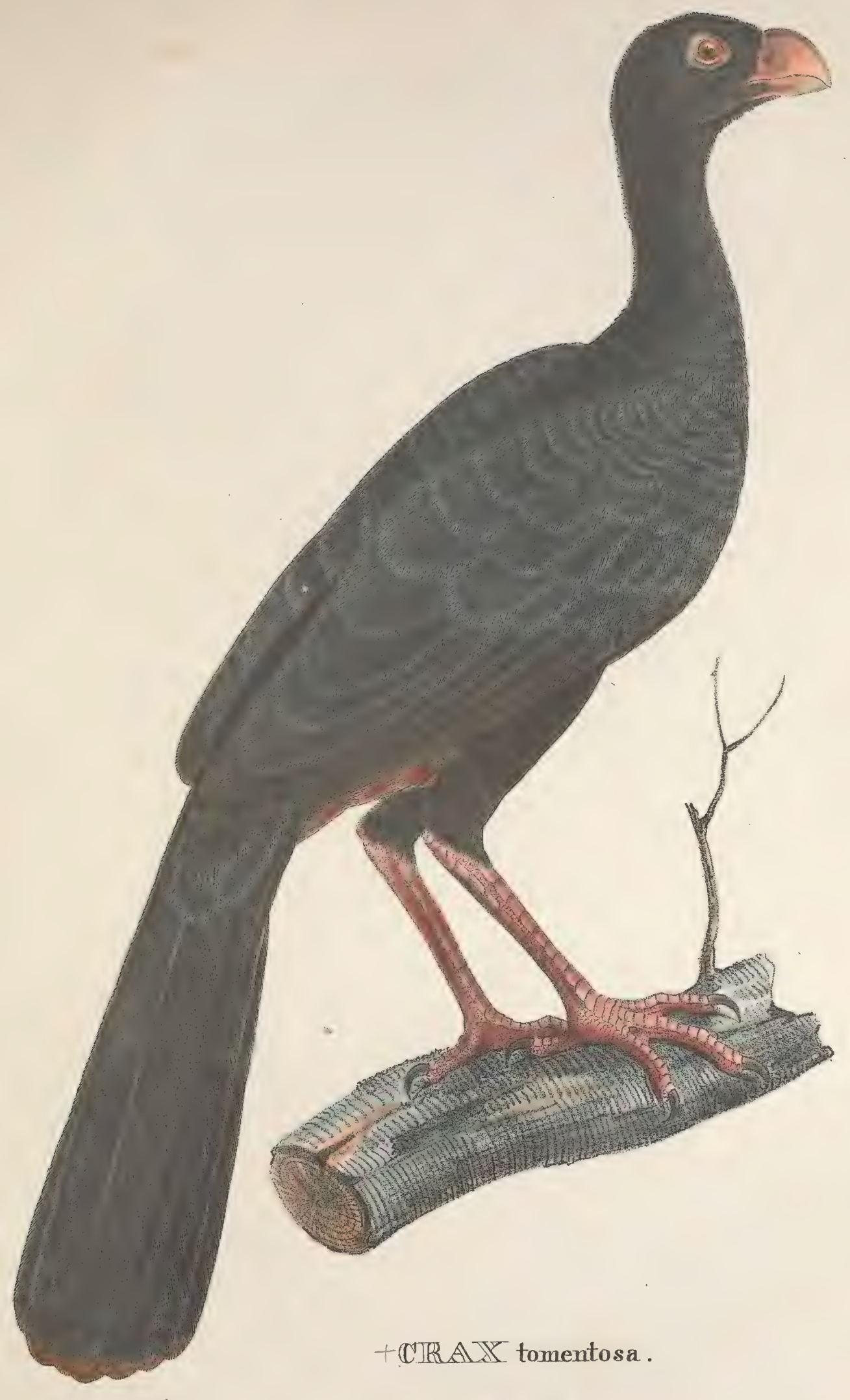

Tab:LXIII . 



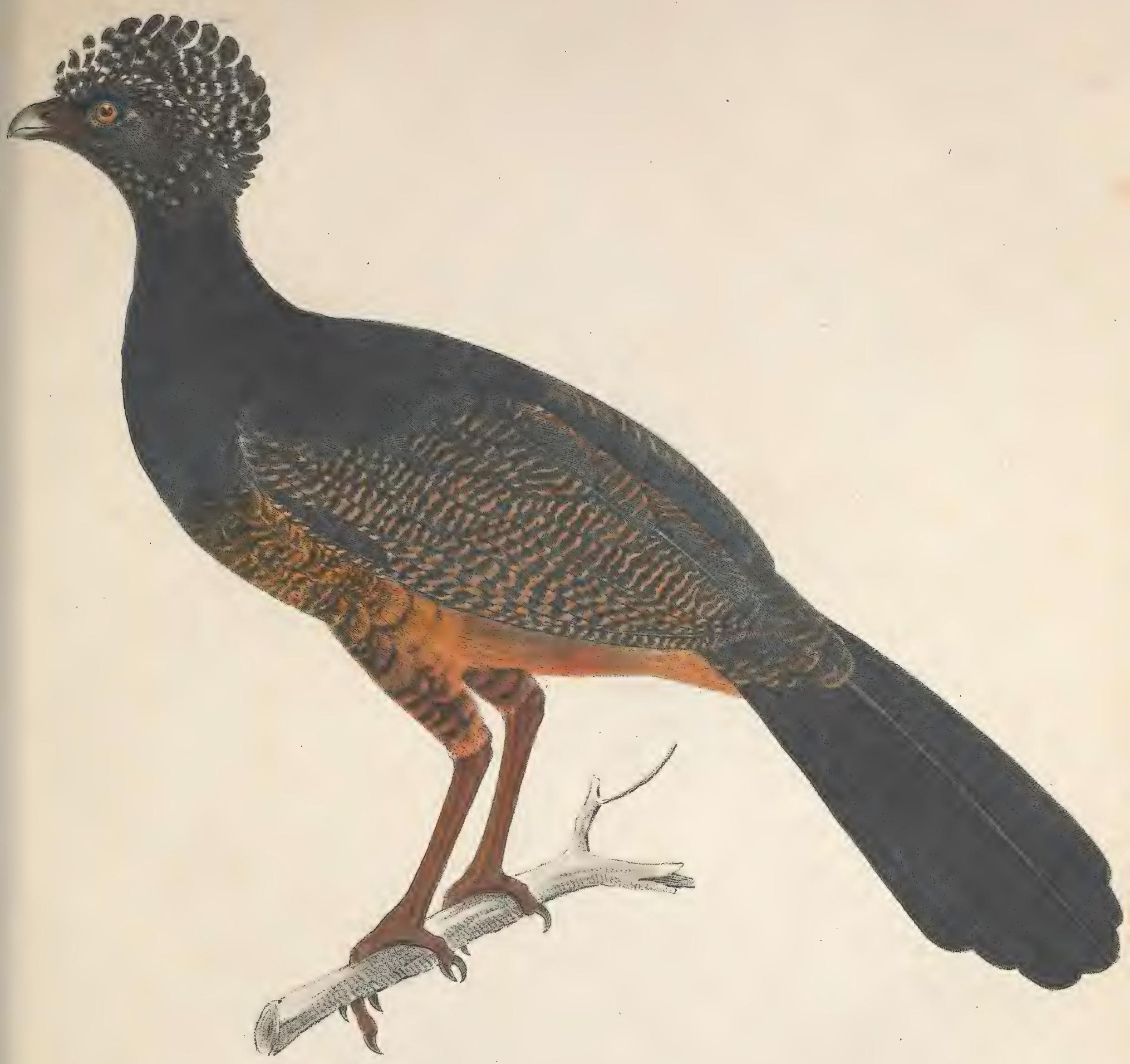

$+\mathbb{C} \mathbb{R} A \mathbb{X}$ Blumenbachii.

Tab. LXIV. 

Sib!s?
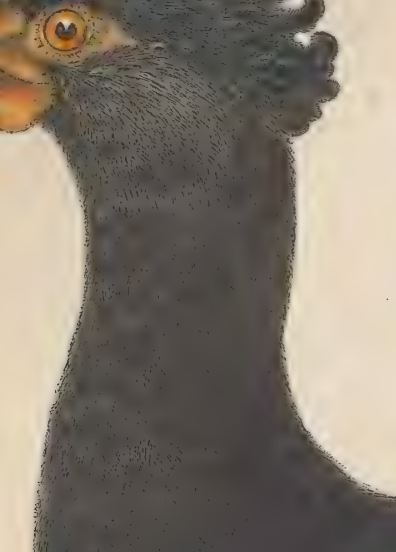



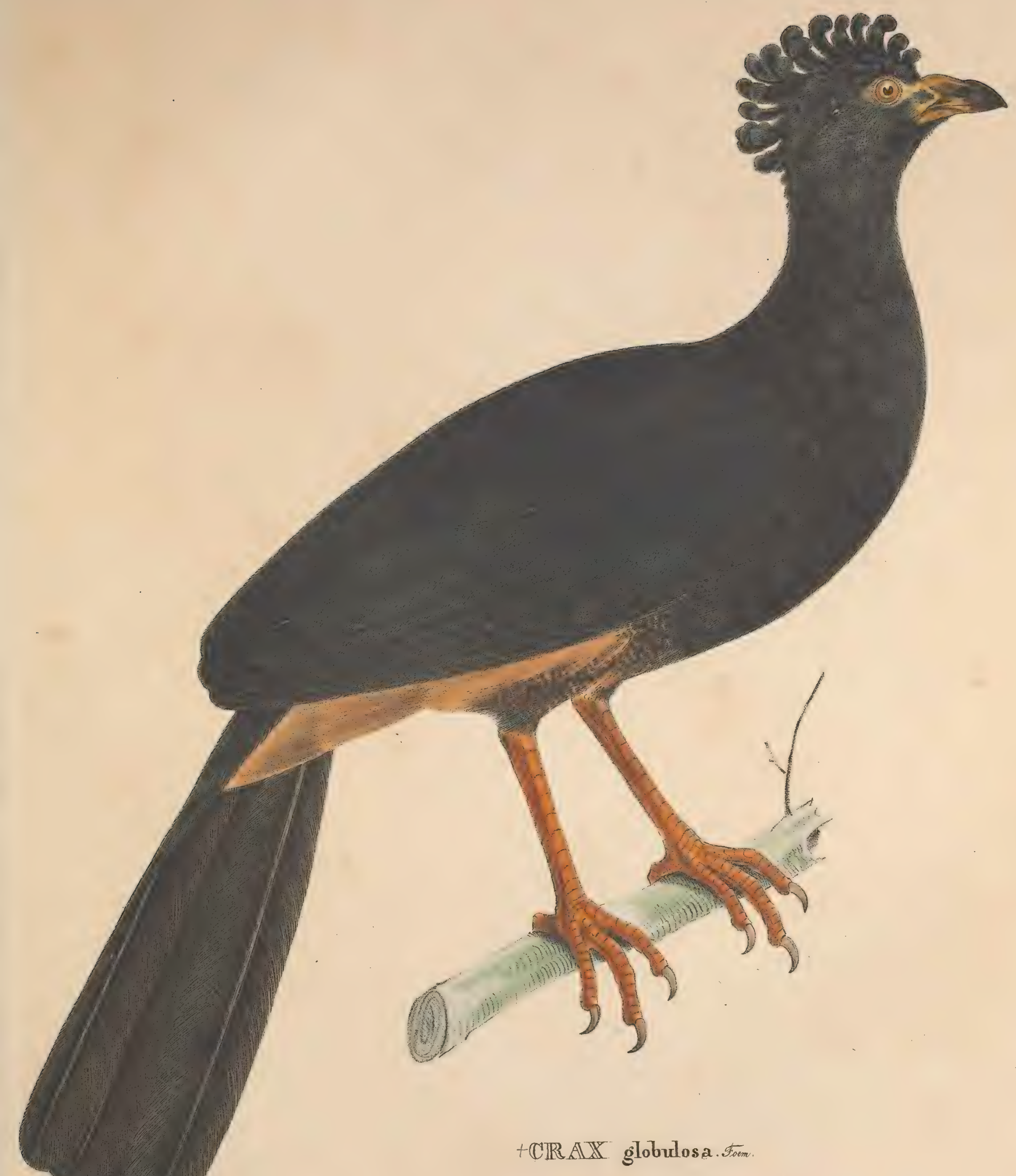

Tab. LXVI. 



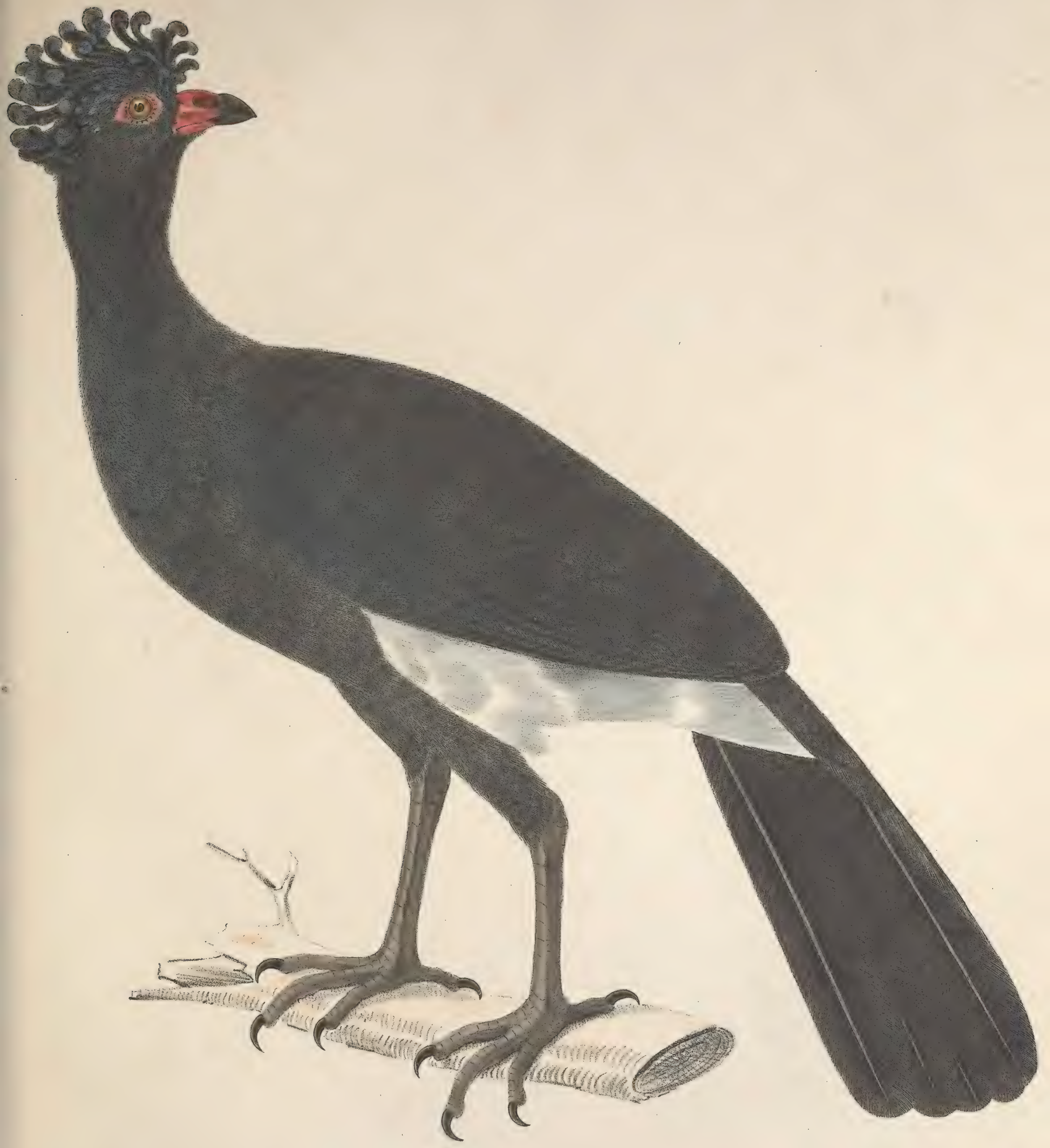

$+\mathbb{C} \mathbb{R} A \mathbb{X}$ Rubrirostris.

Tab.ILXVII. 



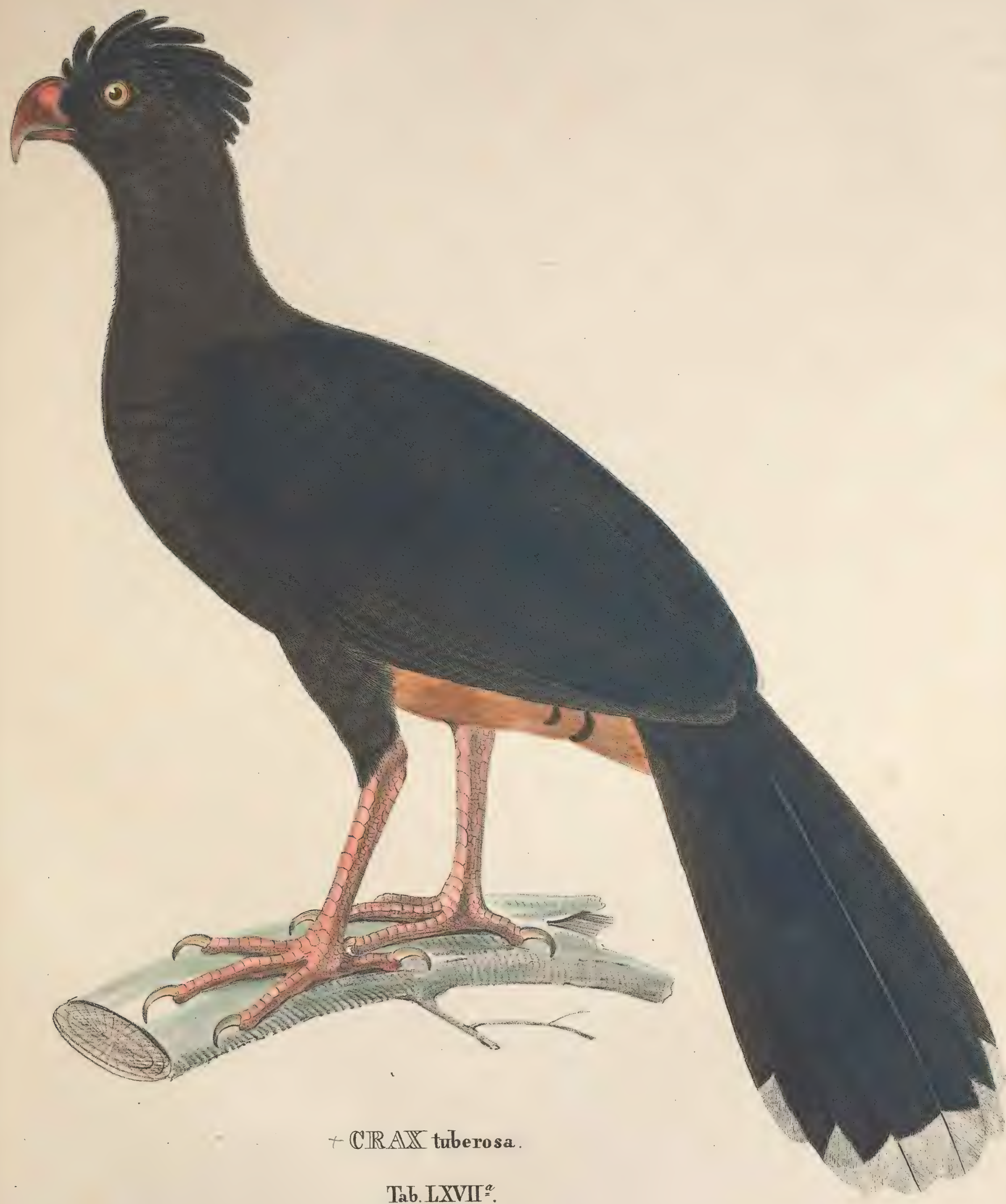





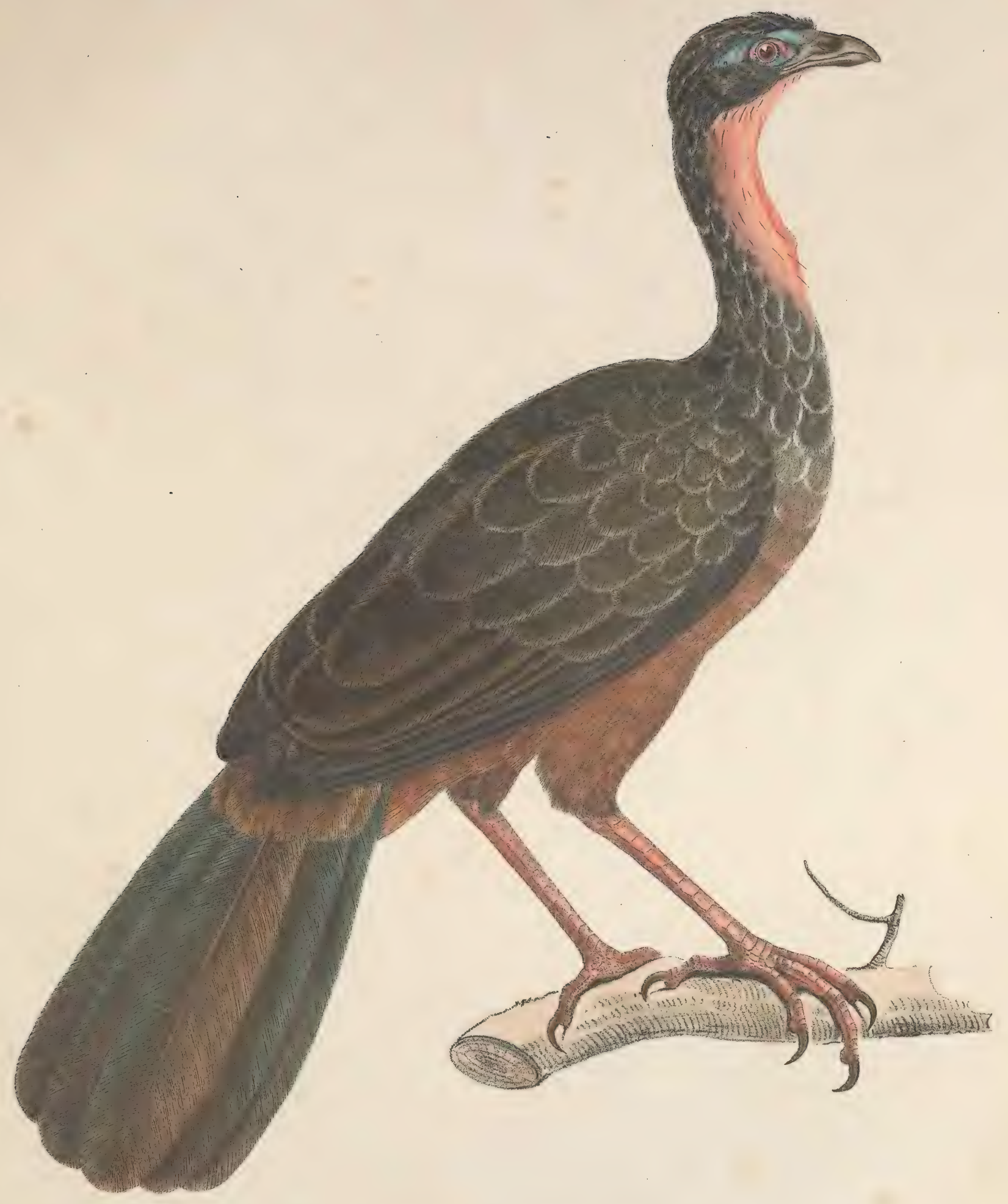

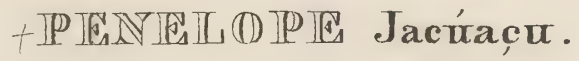

Tab. LXXVIII . 



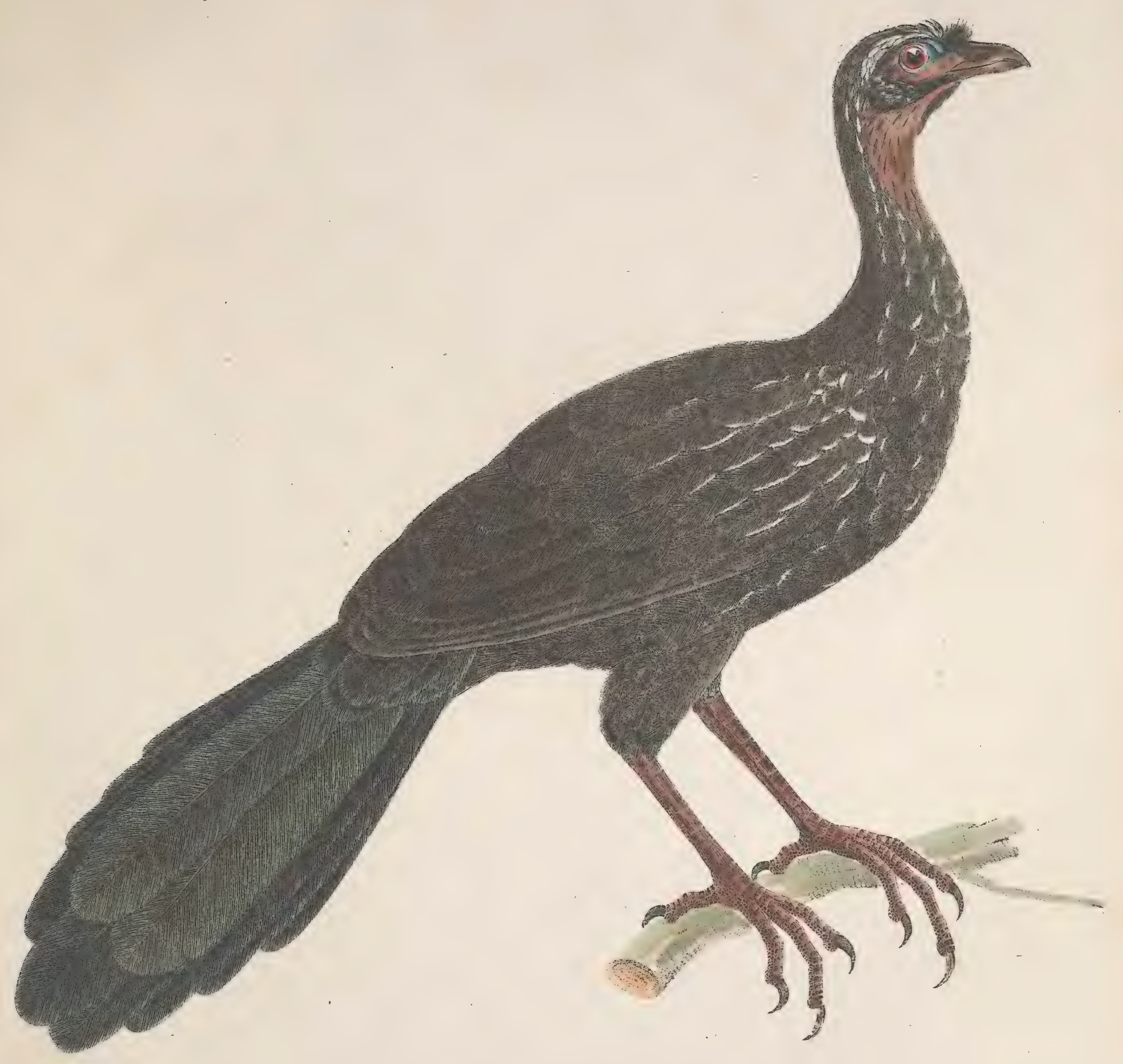

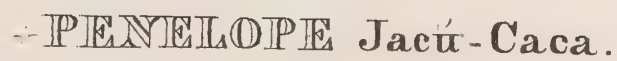

Tab.LXIX . 



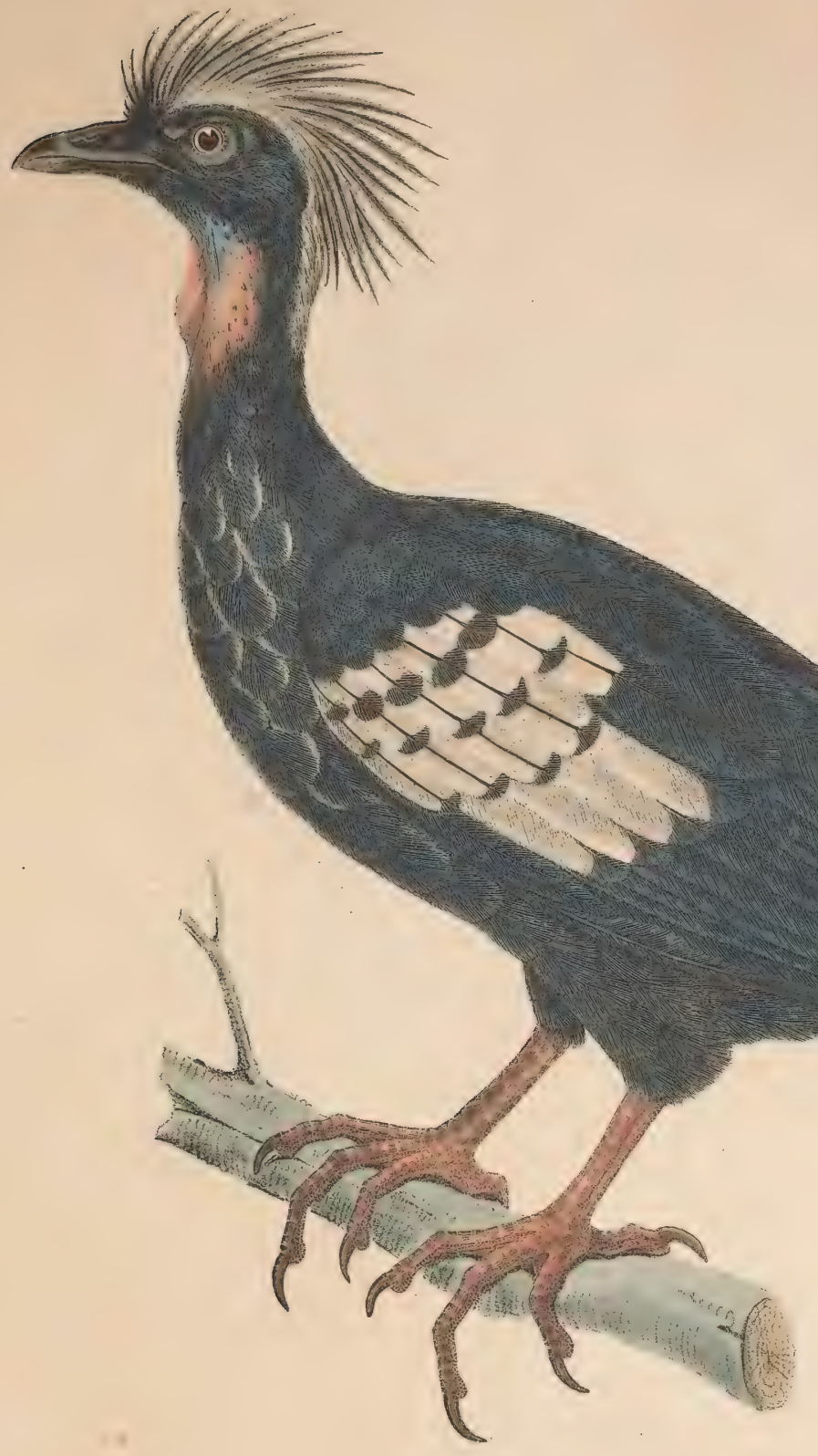

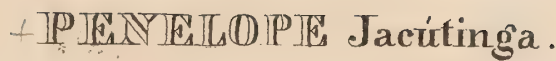

Tab.LXX. 



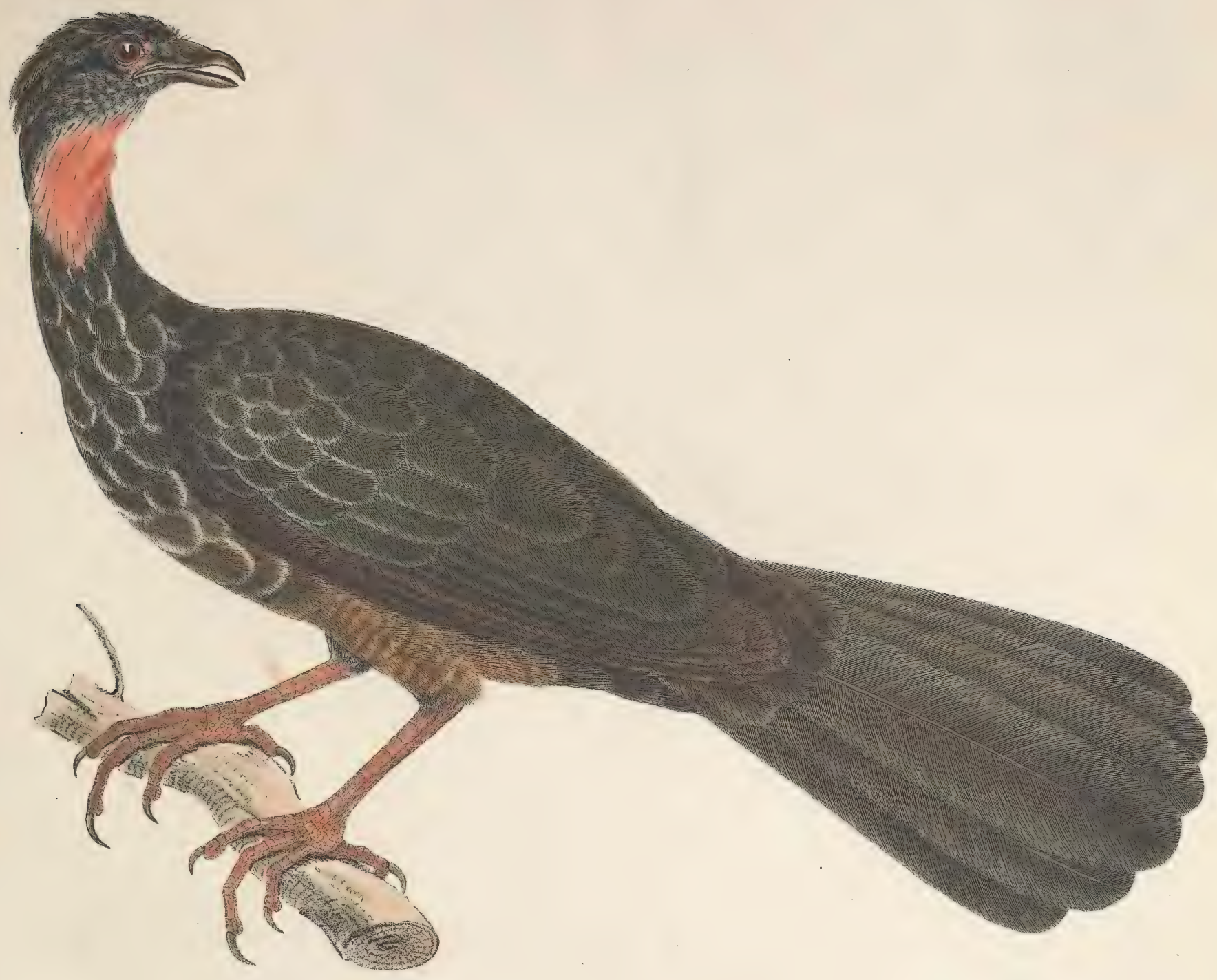

- PIENIEIDAPE Jacú-Peba.

Tab.LXXI . 



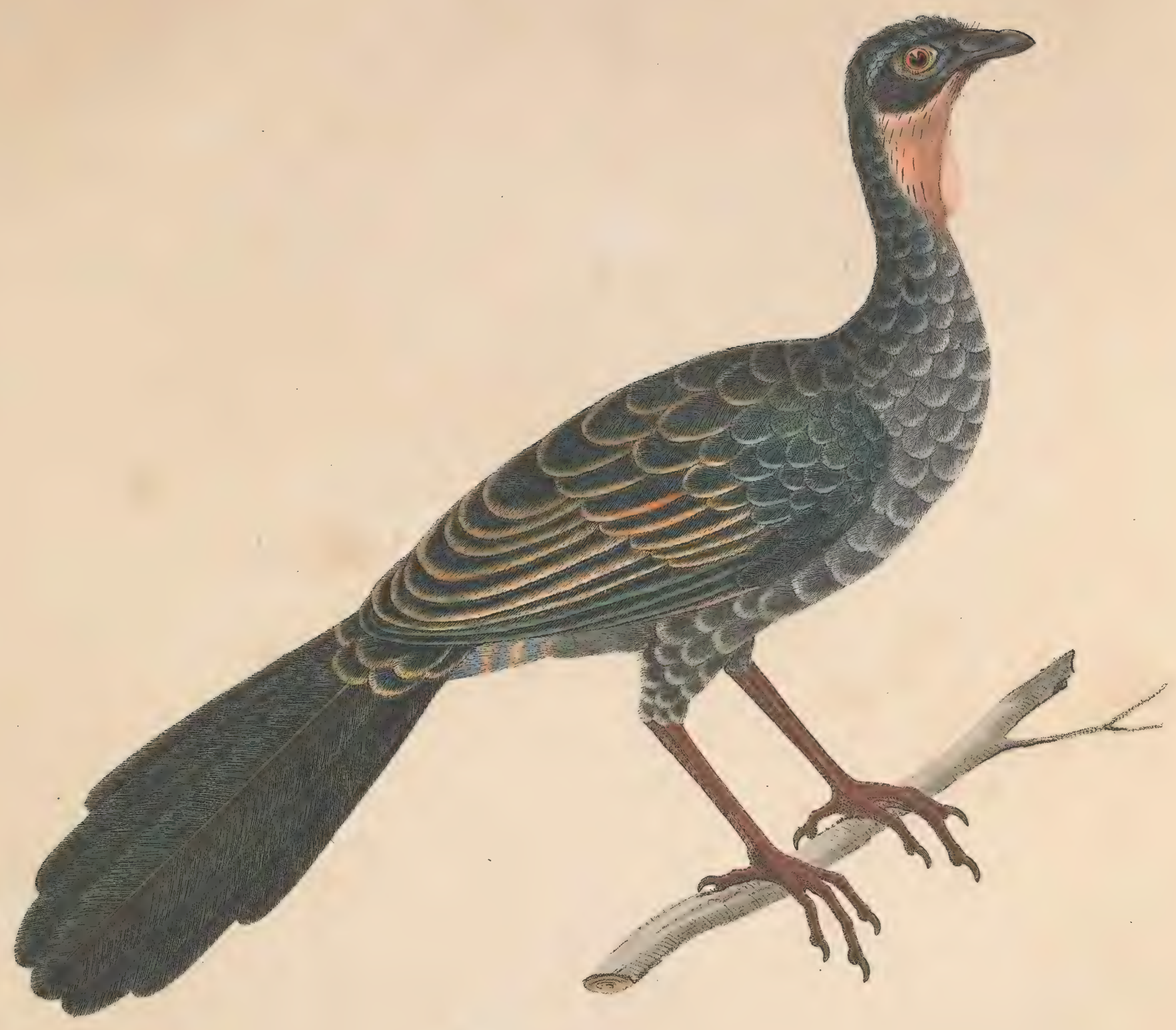

$+\mathbb{P} \mathbb{N} \mathbb{I}_{a} \mathbb{P} \mathbb{P} \mathbb{E}_{a}$ Jacú-Pemba.

Tab.LXXII . 



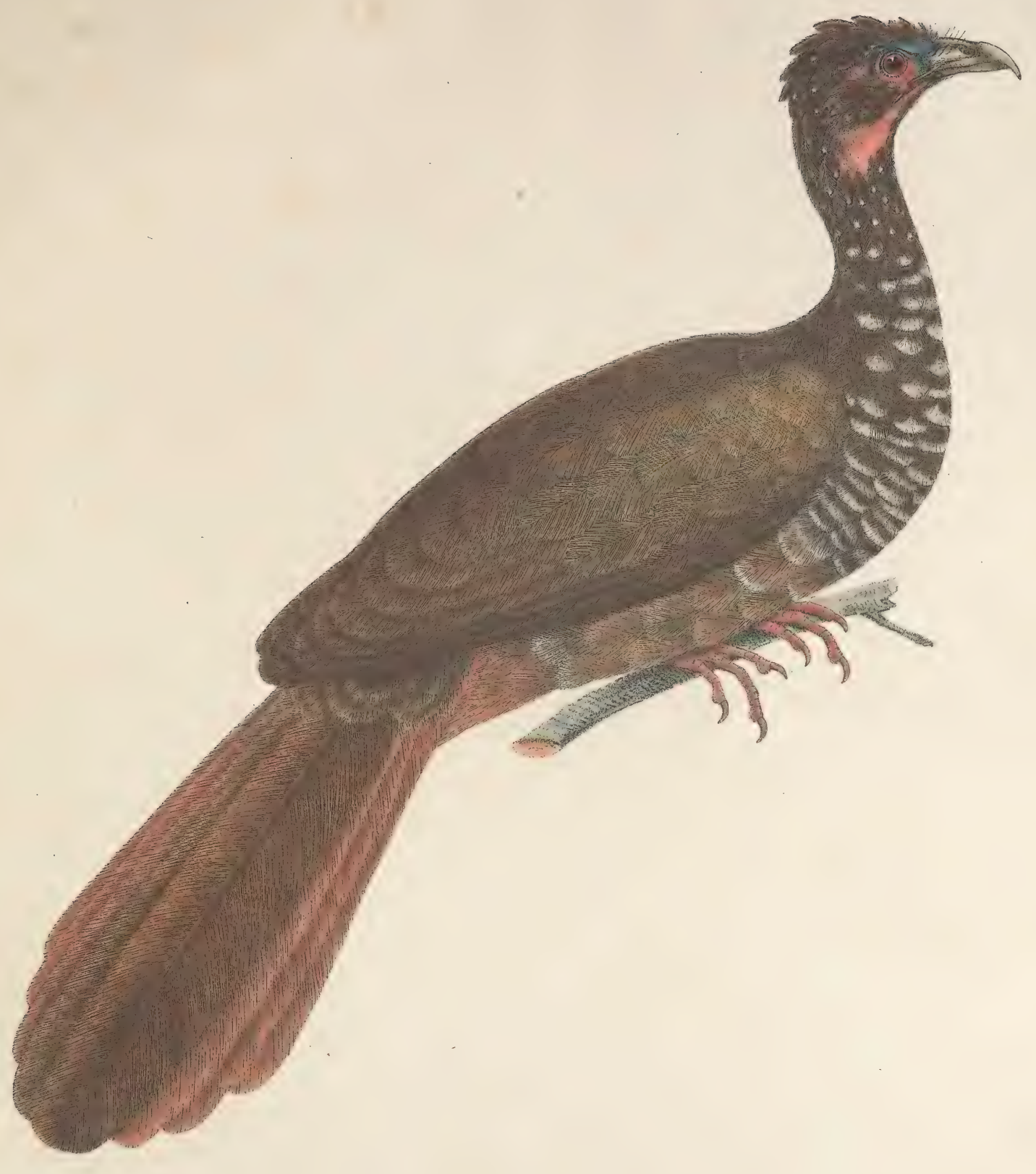

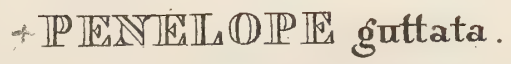

Tab:LXXIII . 



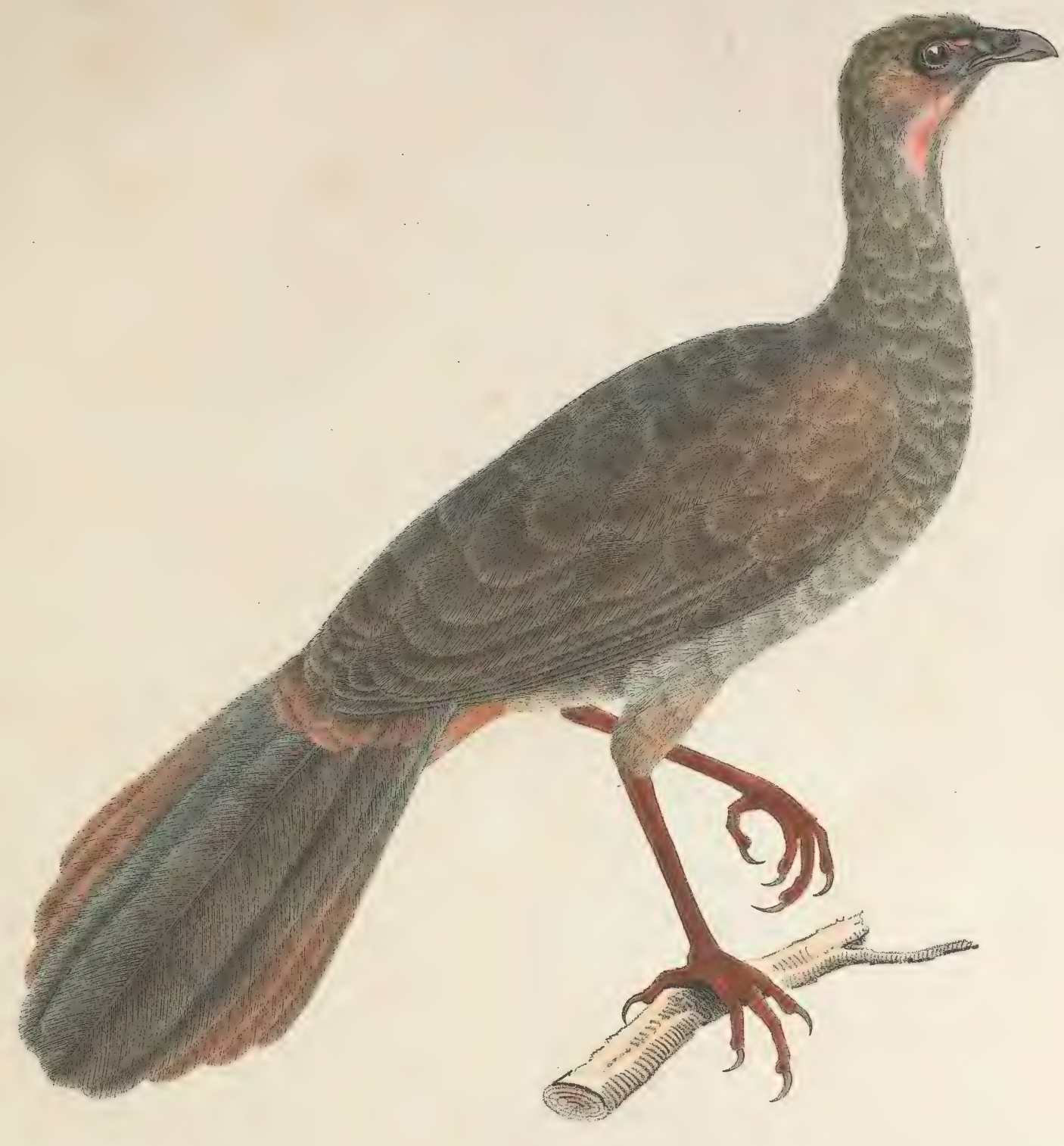

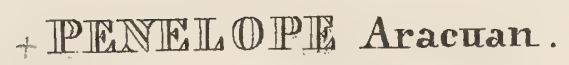

Tab : L XXIV. 


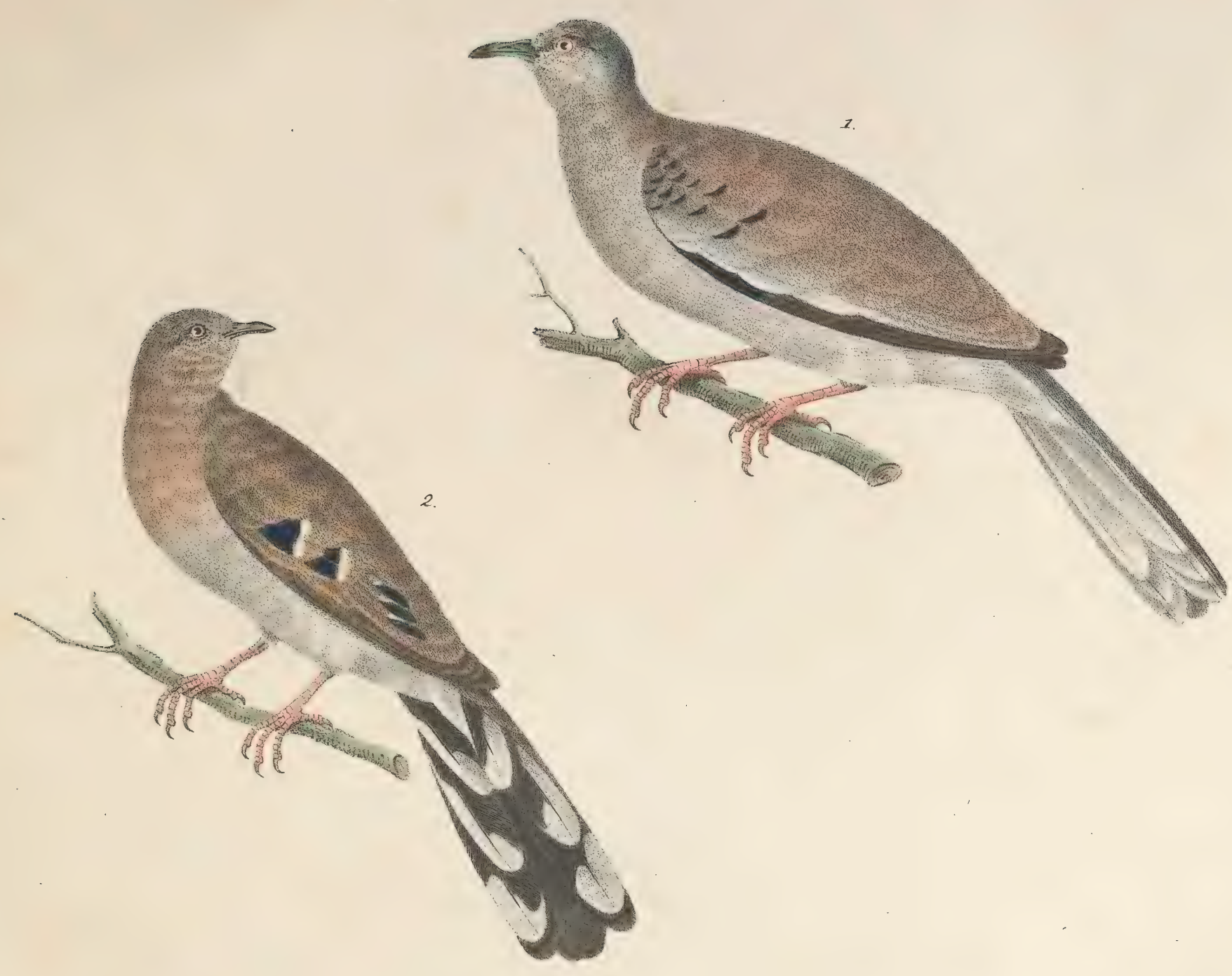

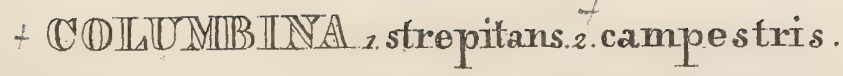

Tab. LXXV. 


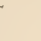



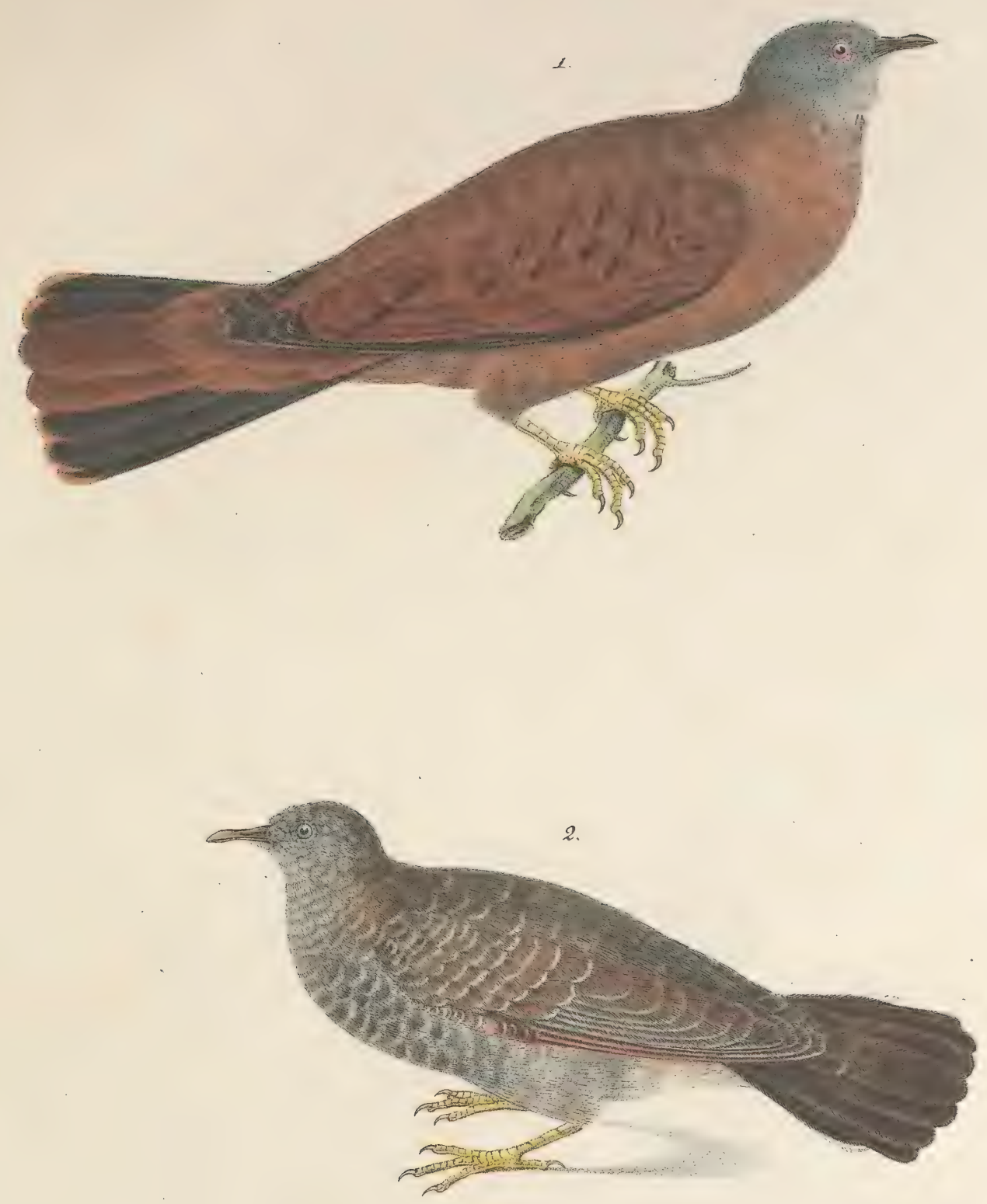

+ $\mathbb{C}(1) H_{4} I O M M I B I N A A_{2}$ Cabocolo. $2^{t}$ griseola.

Tab.LXXV $\stackrel{a}{=}$ 


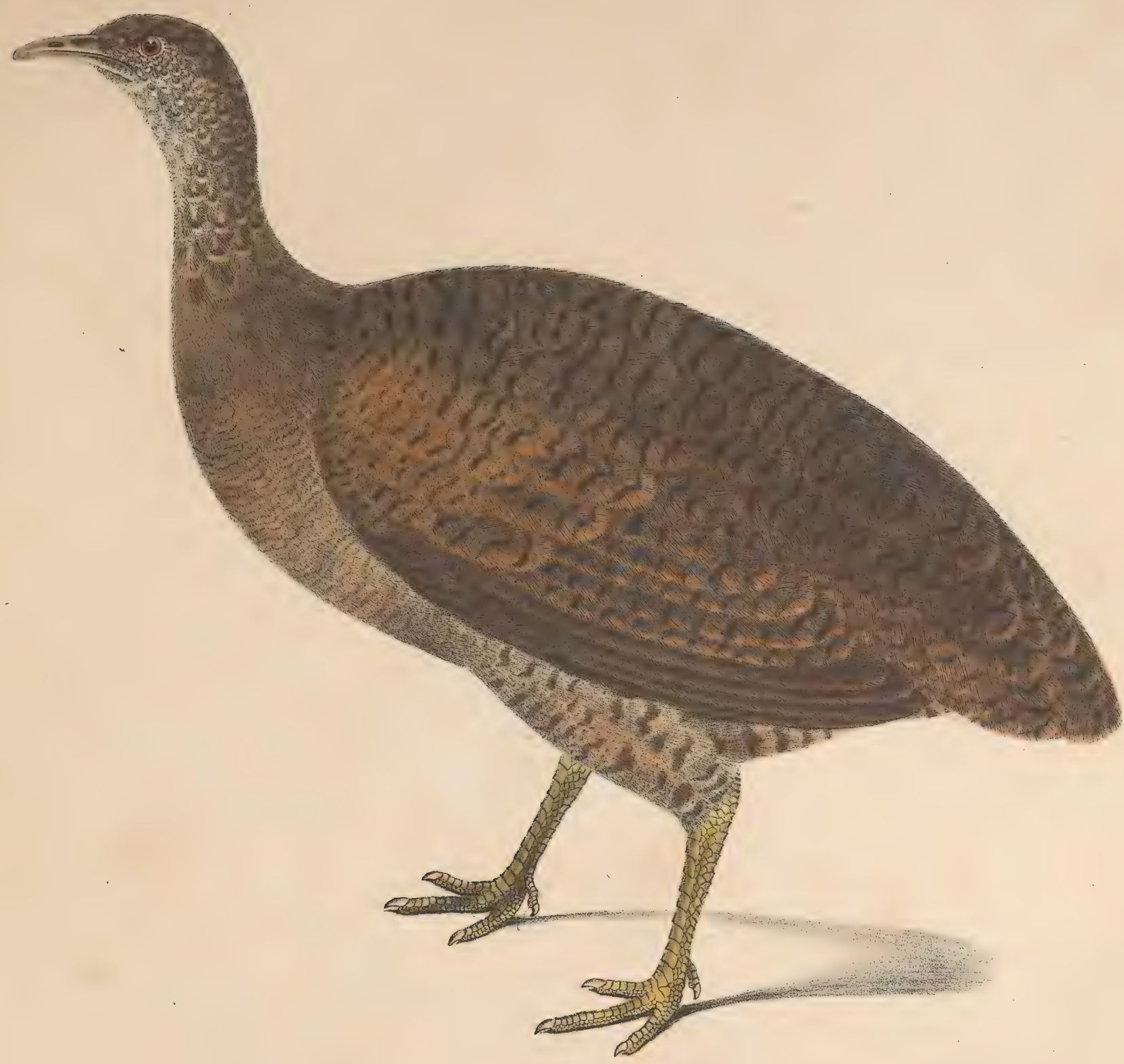

$\perp \mathbb{P} \mathbb{E} \mathbb{Z} \mathbb{U} \mathbb{S}_{S}$ serratus

Tab:LXXVI . 



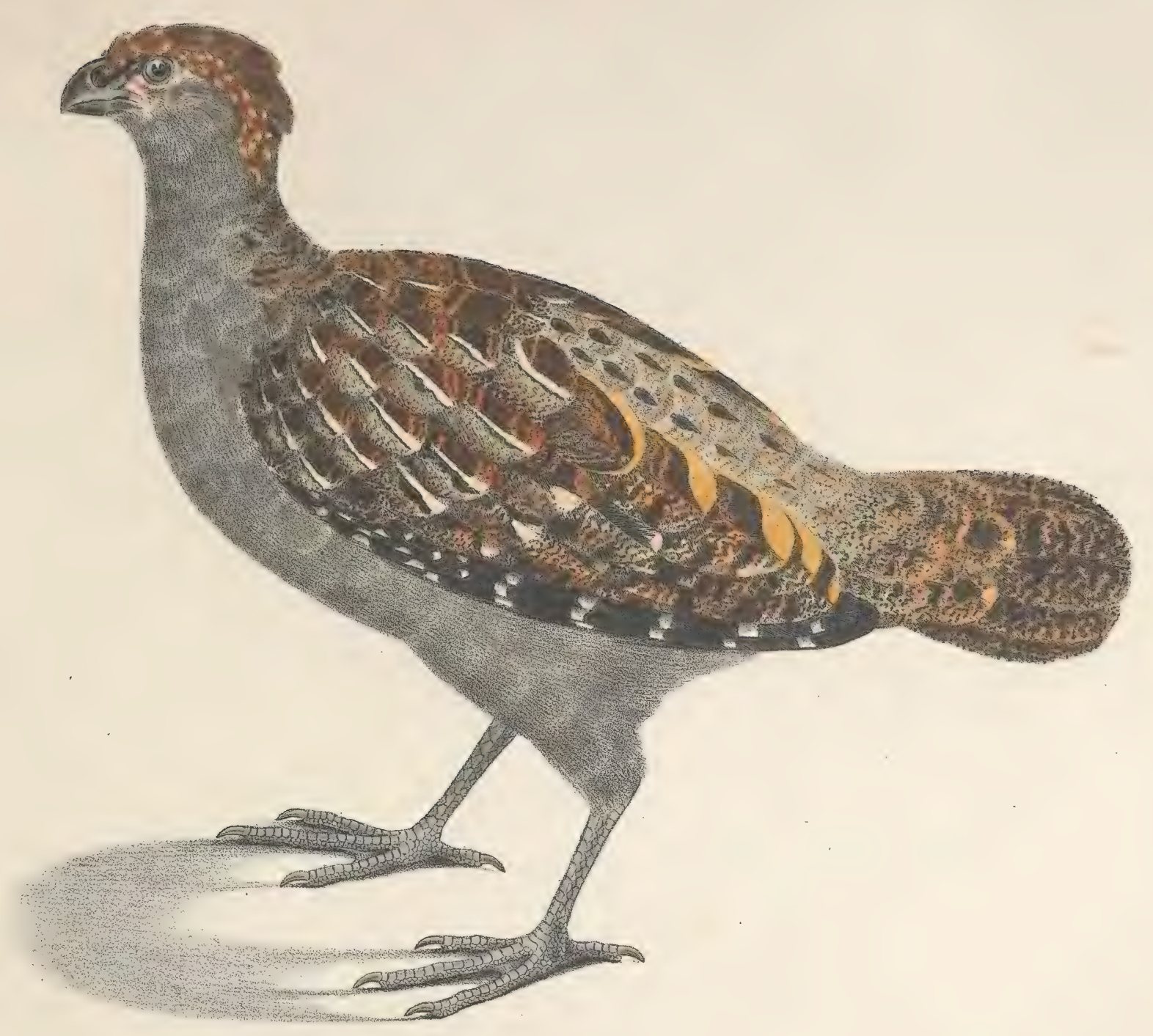

$+\mathbb{P} I F] R$ IDTL Capueira

$\mathrm{T} a b$ :LXXVI $\stackrel{a}{-}$ 



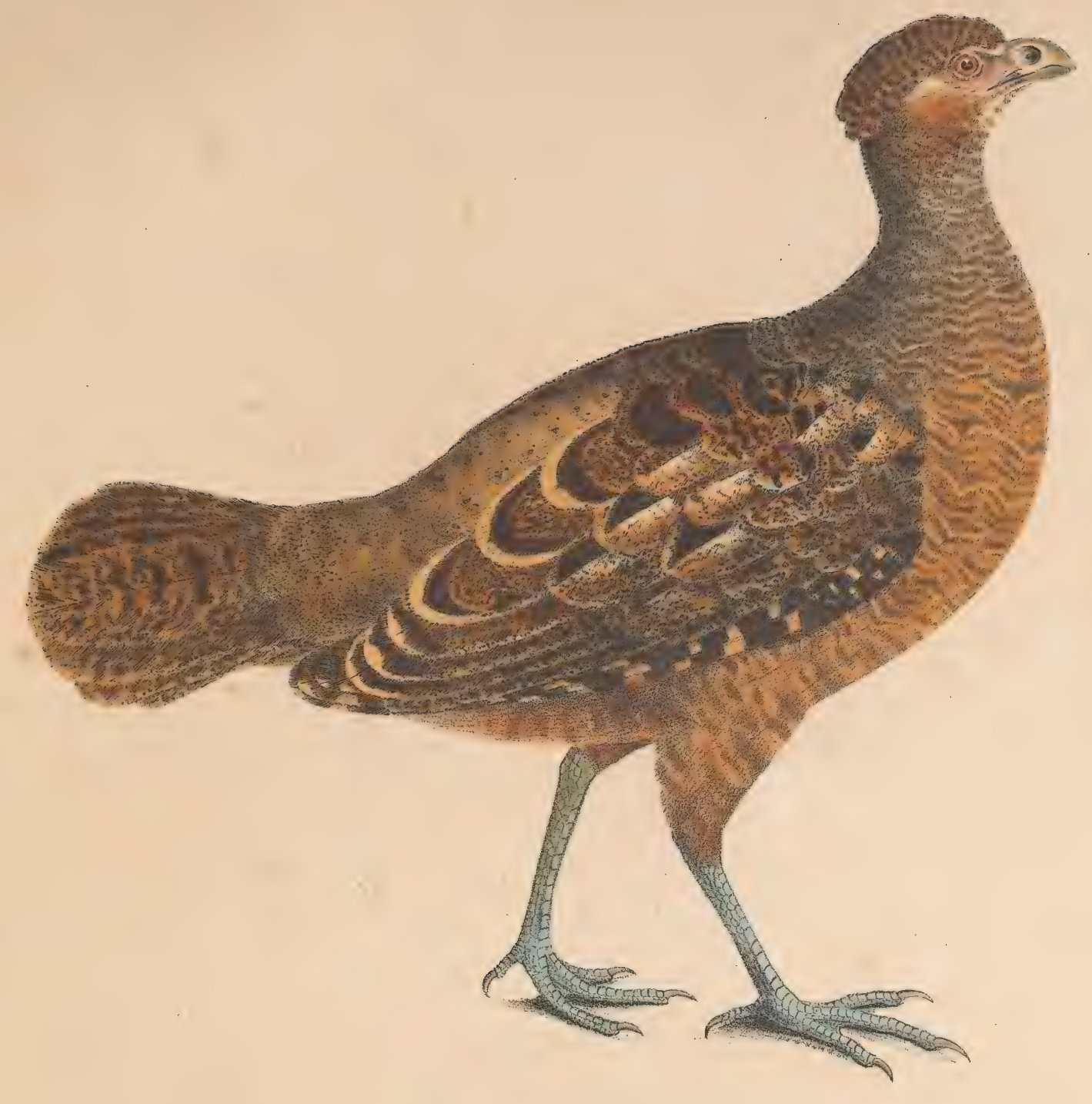

$+P \mathbb{P} T R \mathbb{R} I \mathbb{X}$ rufina.

Tab : LXXVI 



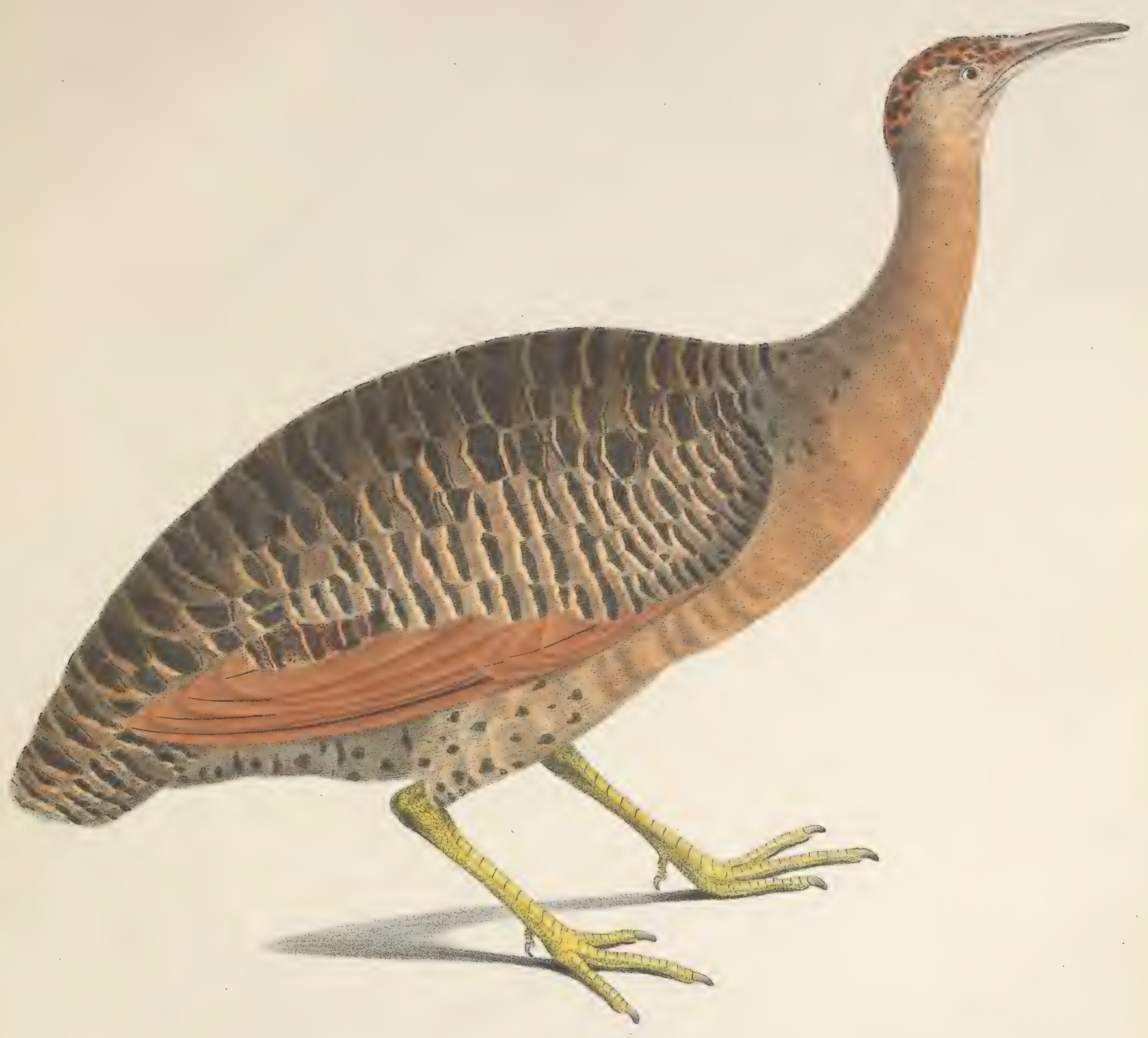

IRTHYNCTEIOTTYS fasciatus.

+Tab.IXXXVI - . 



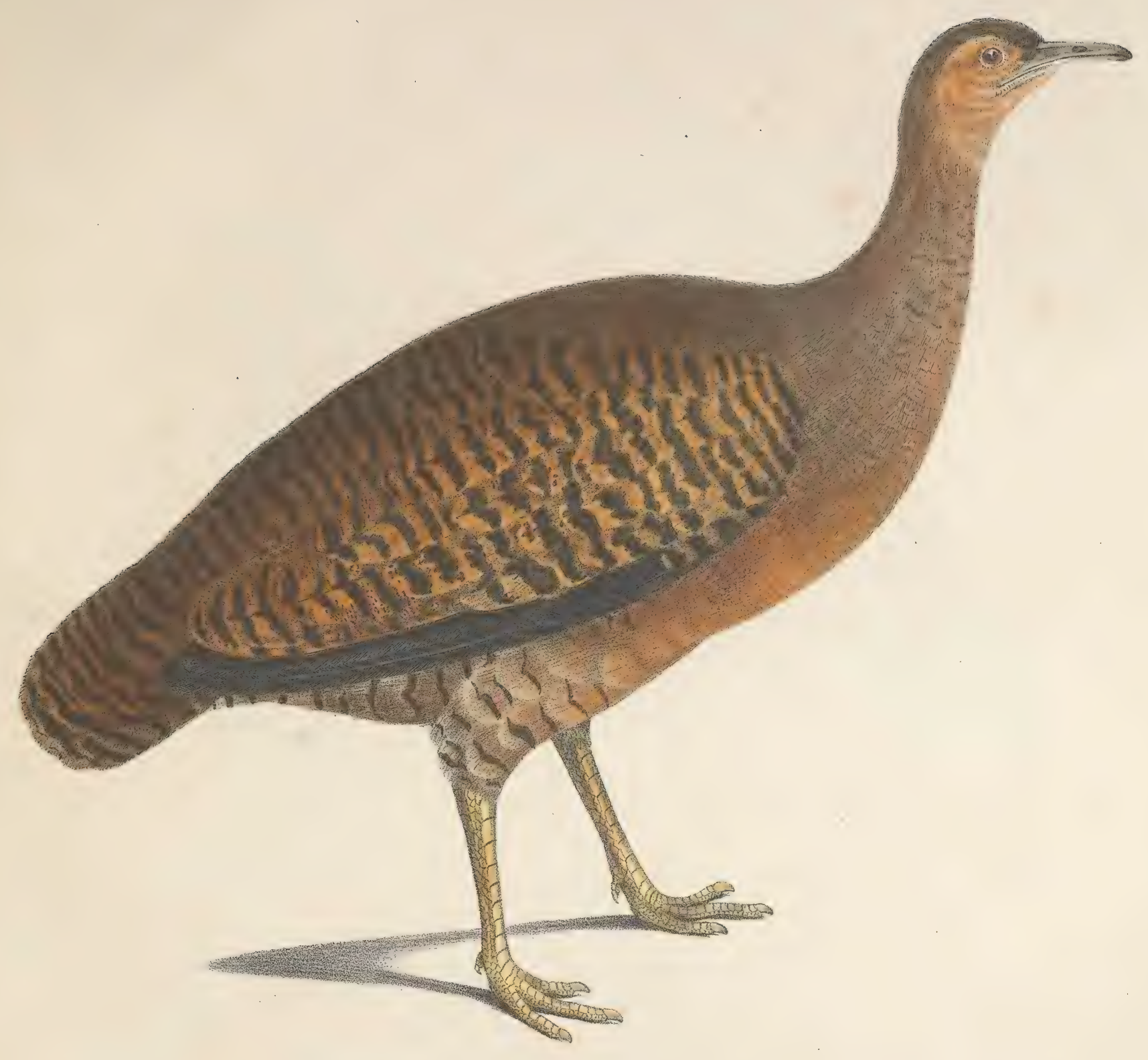

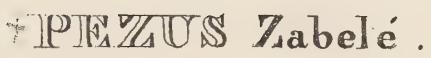

Tab:LXXVII . 



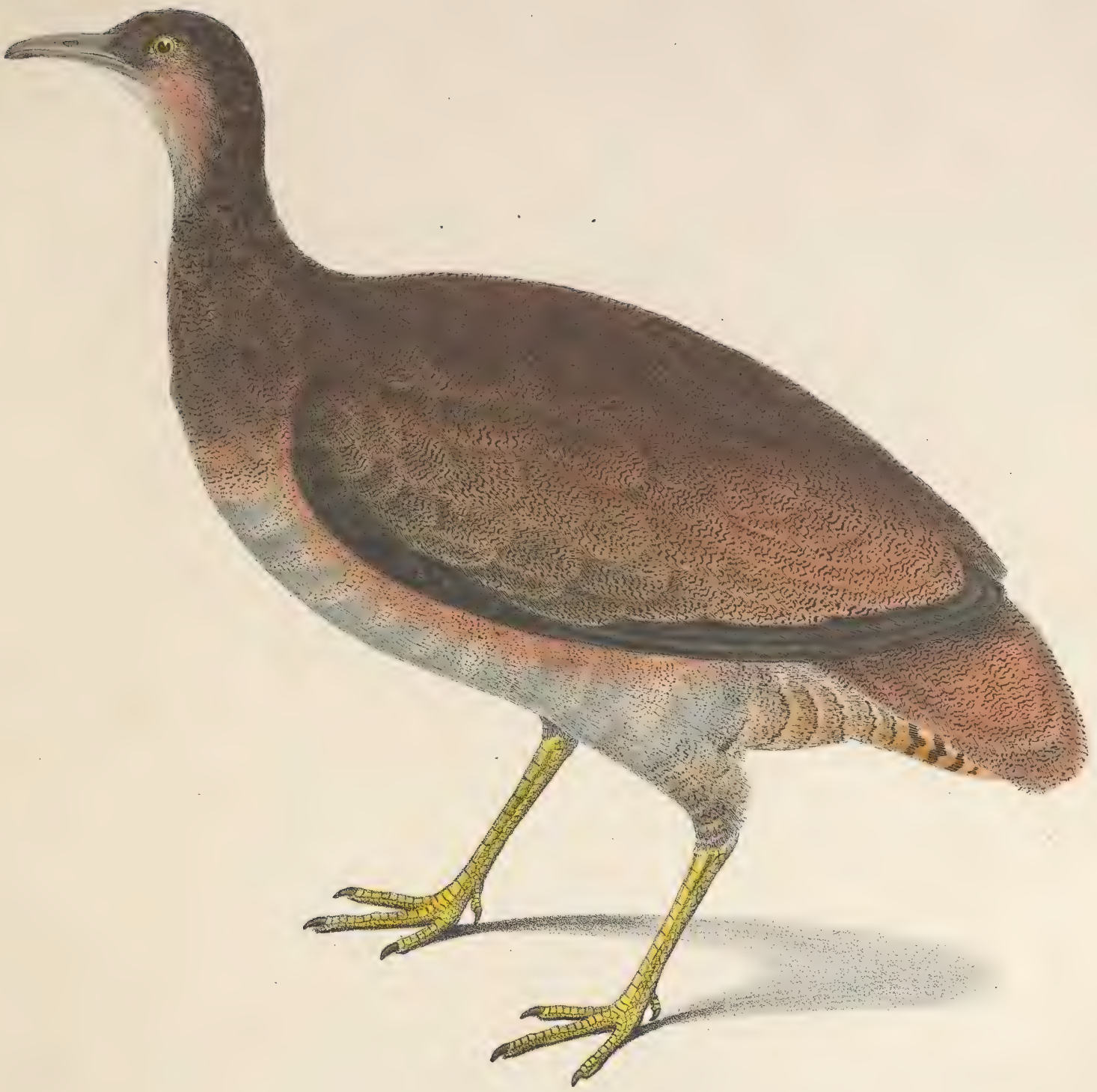

tIP 四US Tapura.

Tab.TXXVIII. 



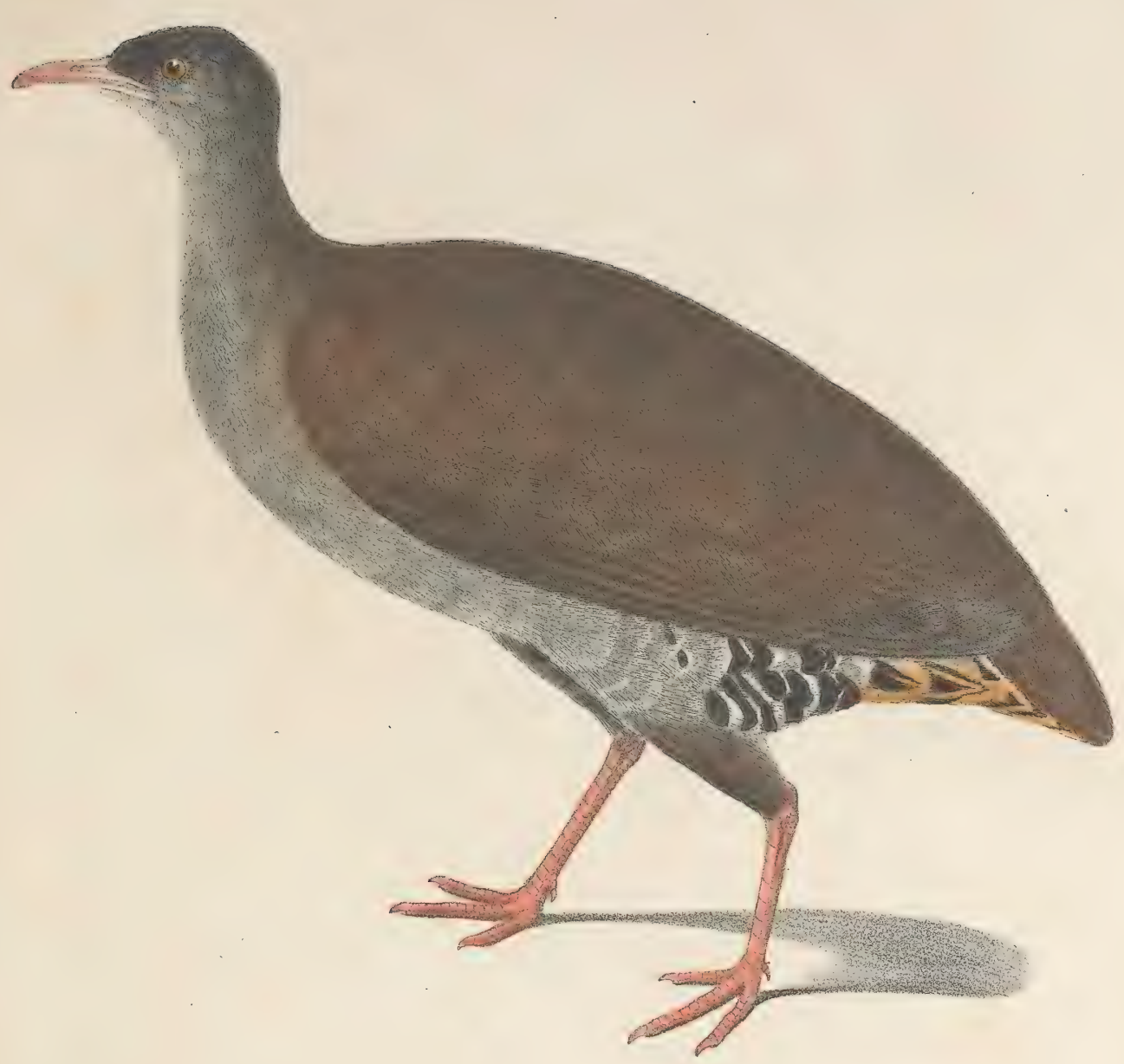

$+\mathbb{P}$ 世

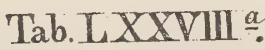





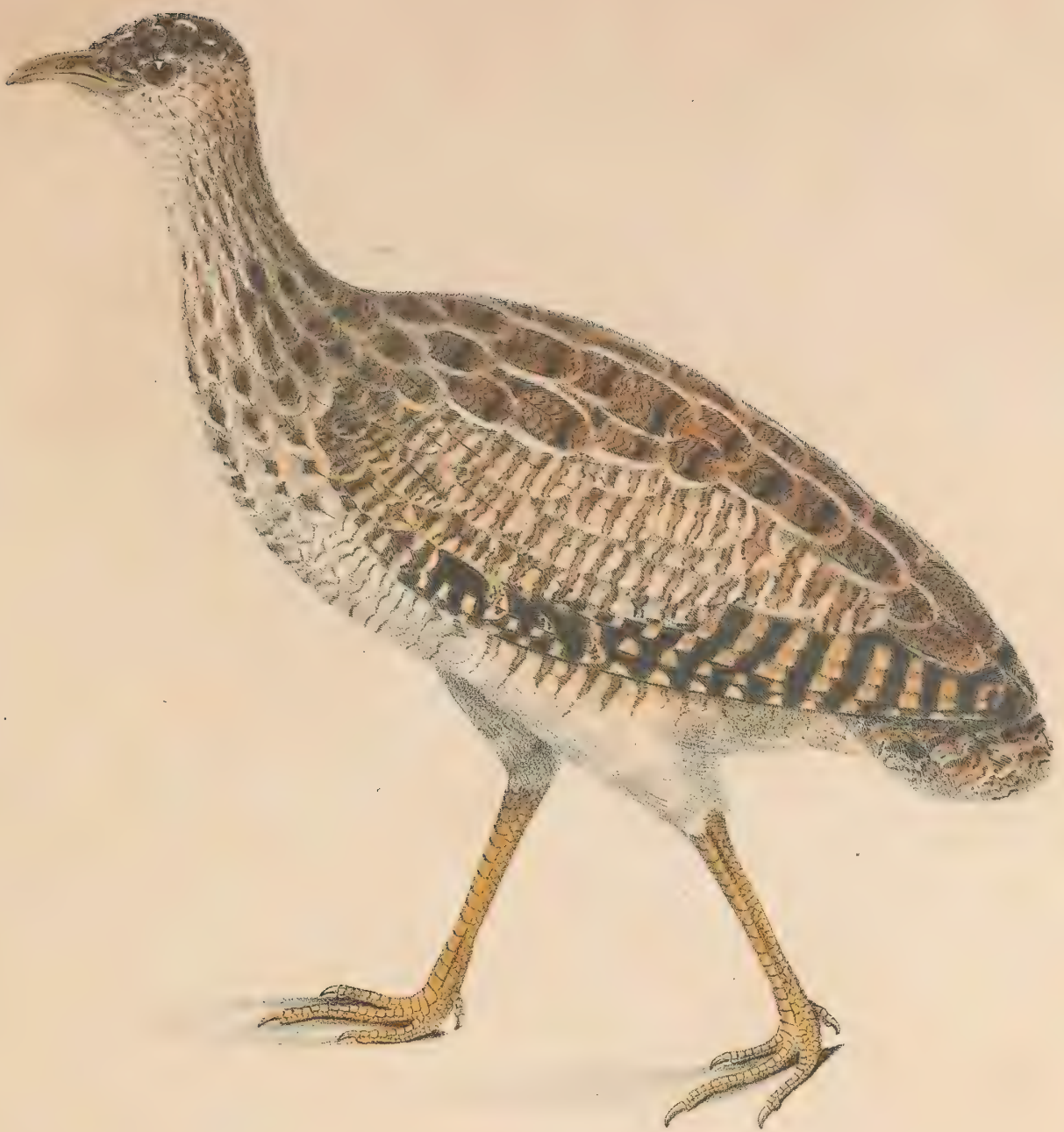

+ TIIINA MUUS Boraquira.

'Jáb:LXXIX . 



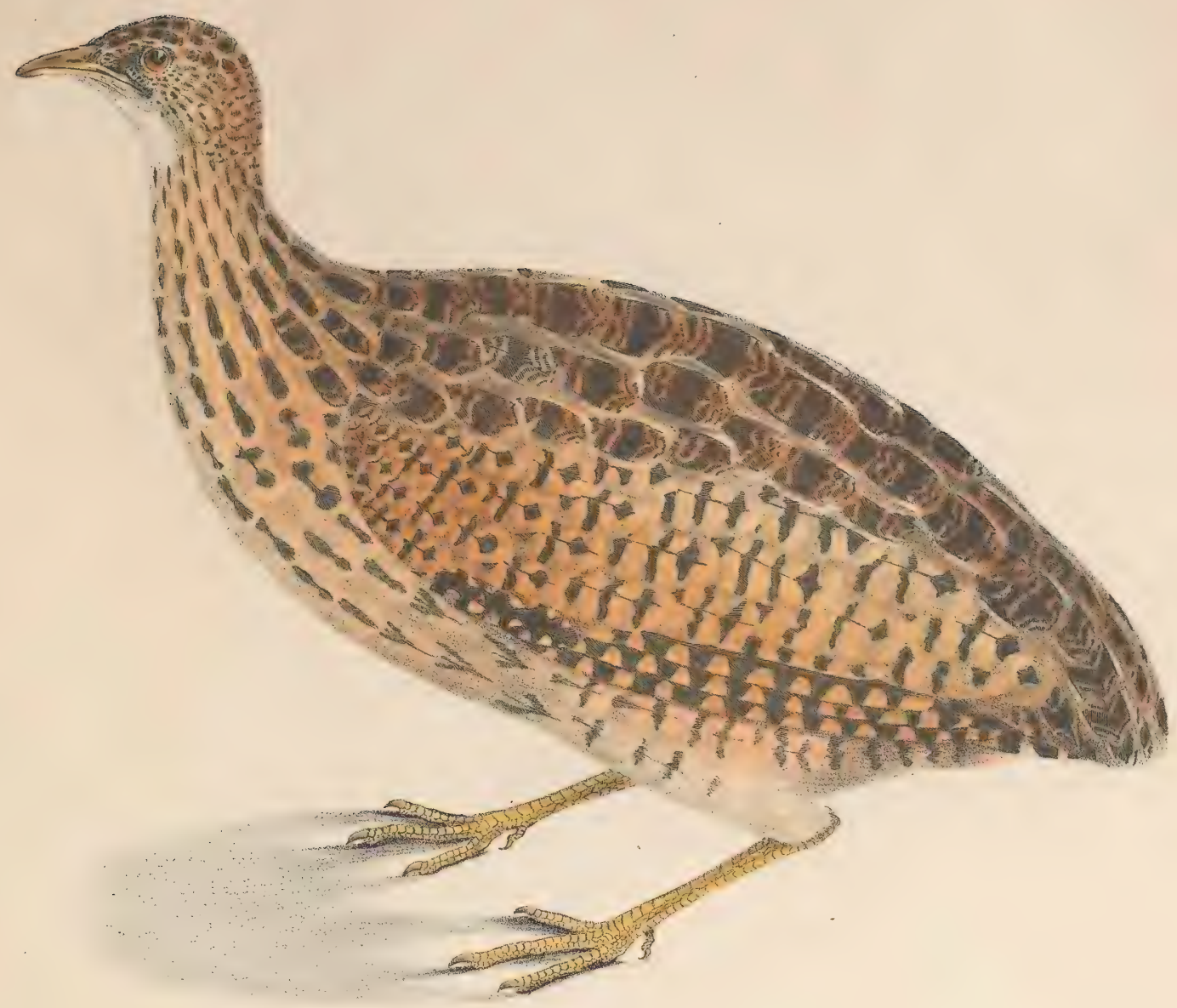

+

T26: LXXX . 



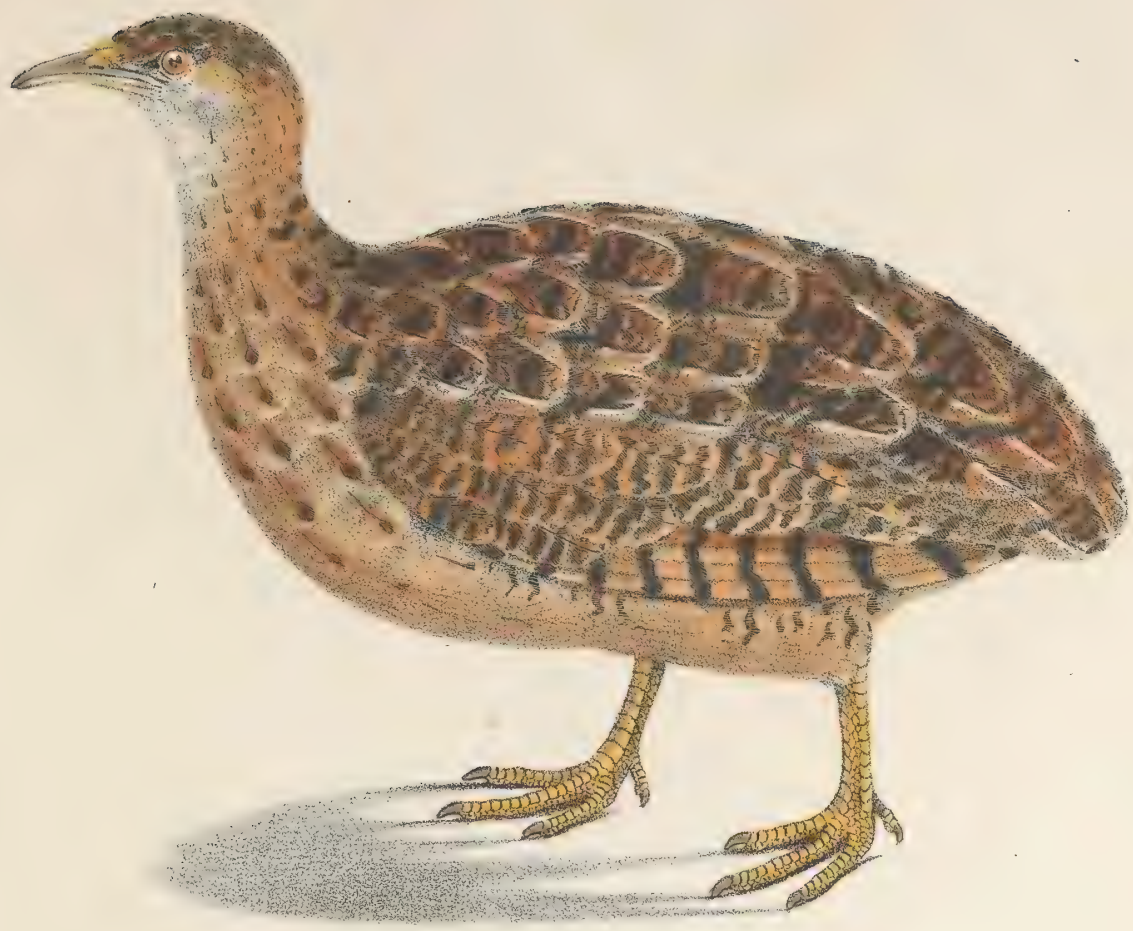

TILNAMTOS medius.

$\mathrm{T} a b: \mathrm{LXXXI}$. 



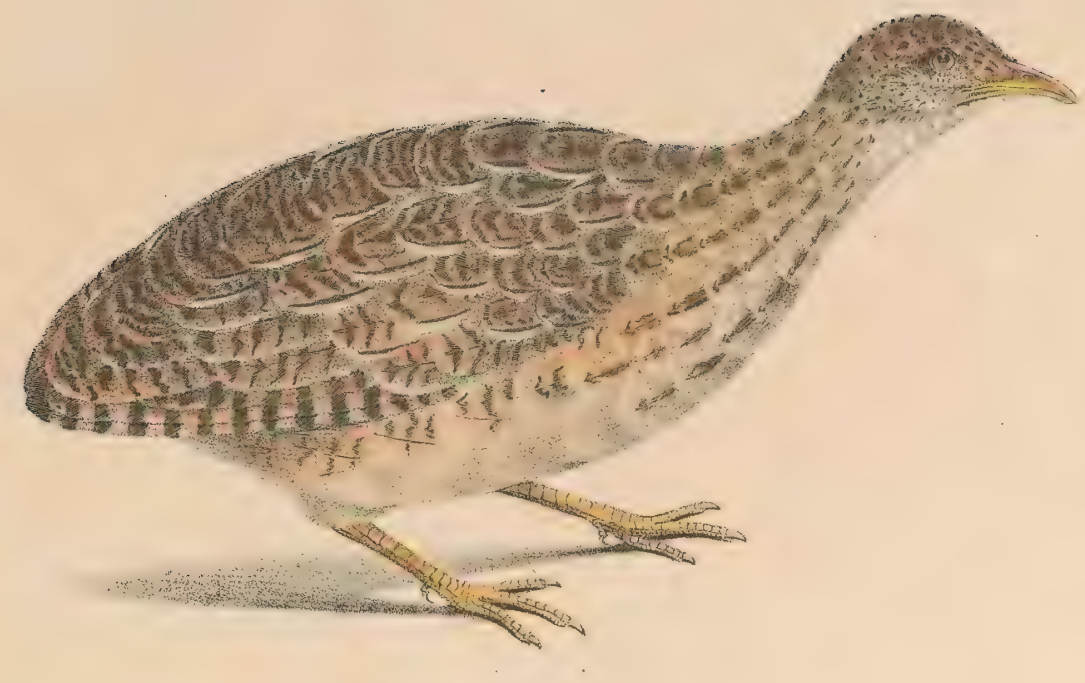

+ PIINA AMIUS minor.

Tab:LXXXII . 



$$
\phi
$$




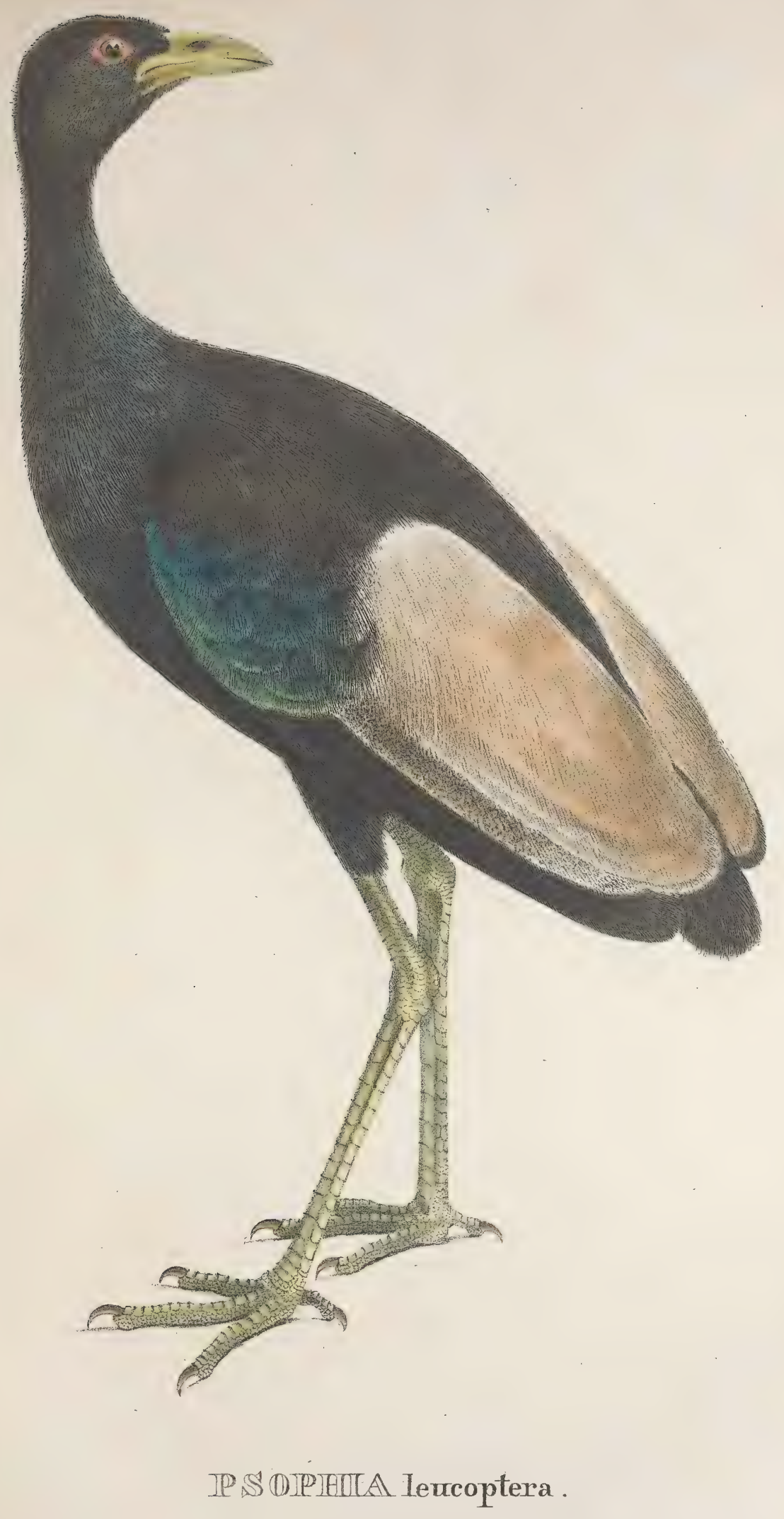

Tab : LXXXTV. 


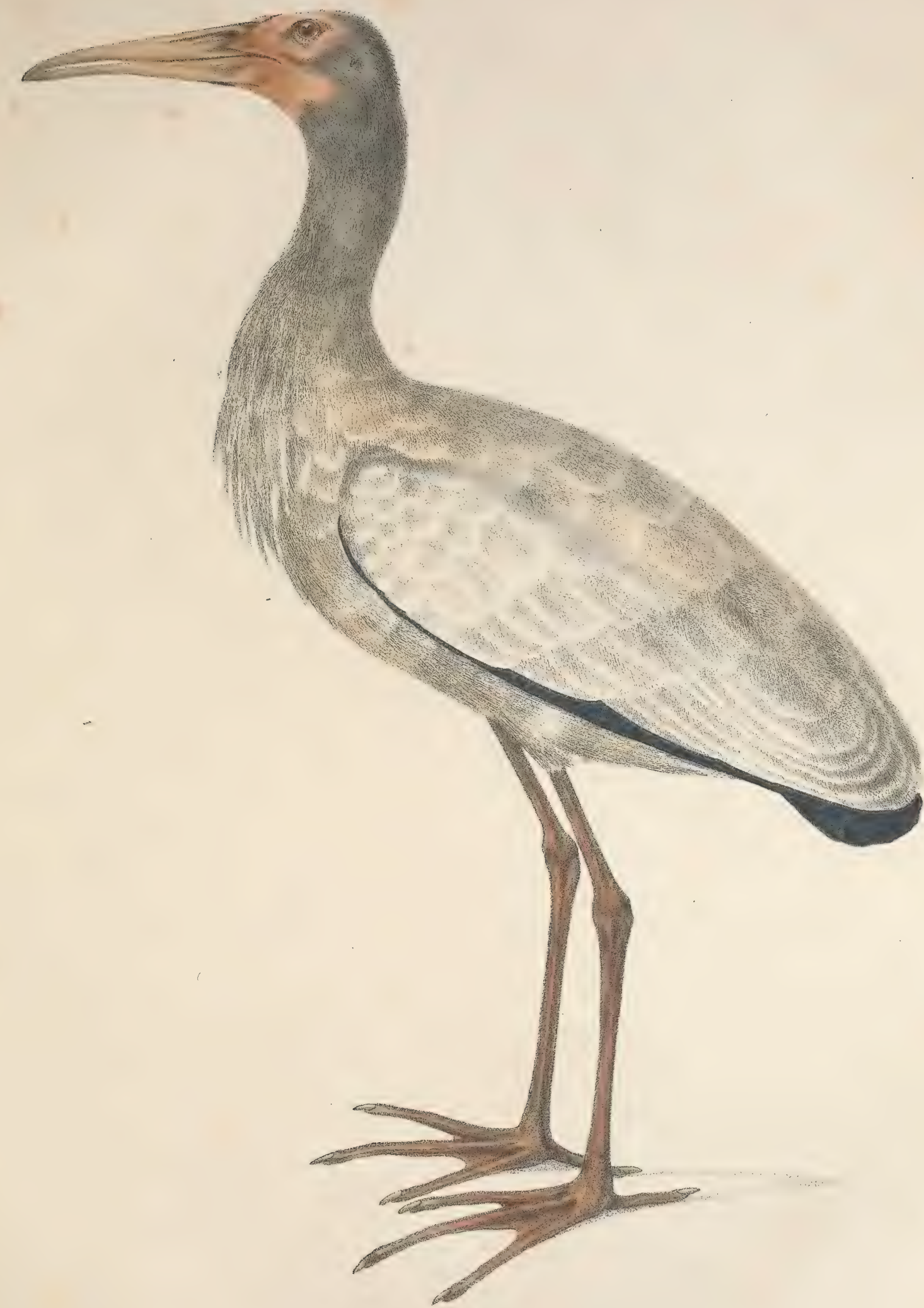

+TANPATIUSS Plumicollis.

Tab.LXXXV 



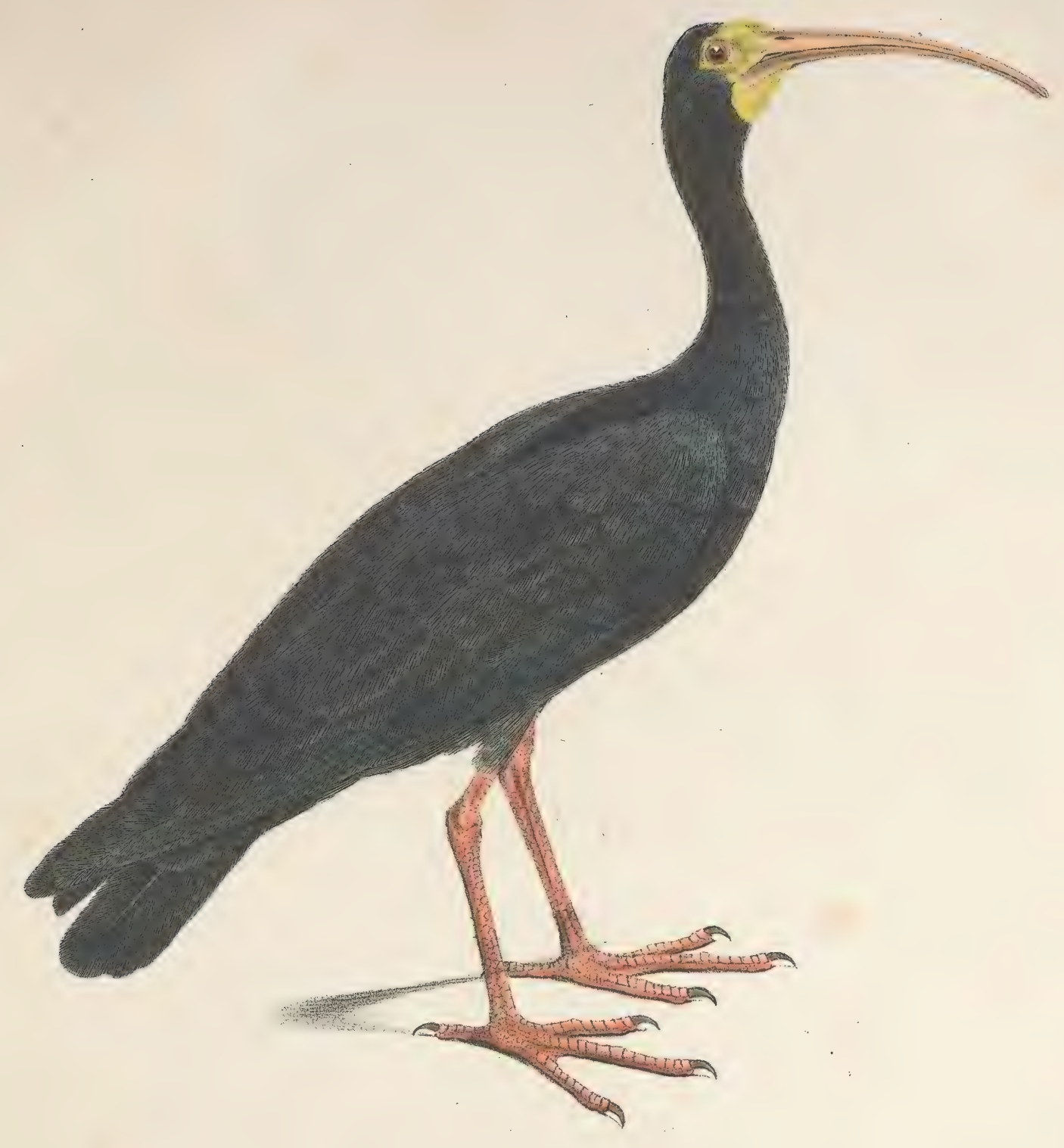

IIIBISS Nudifrons.

Tab:LXXXVT .. 



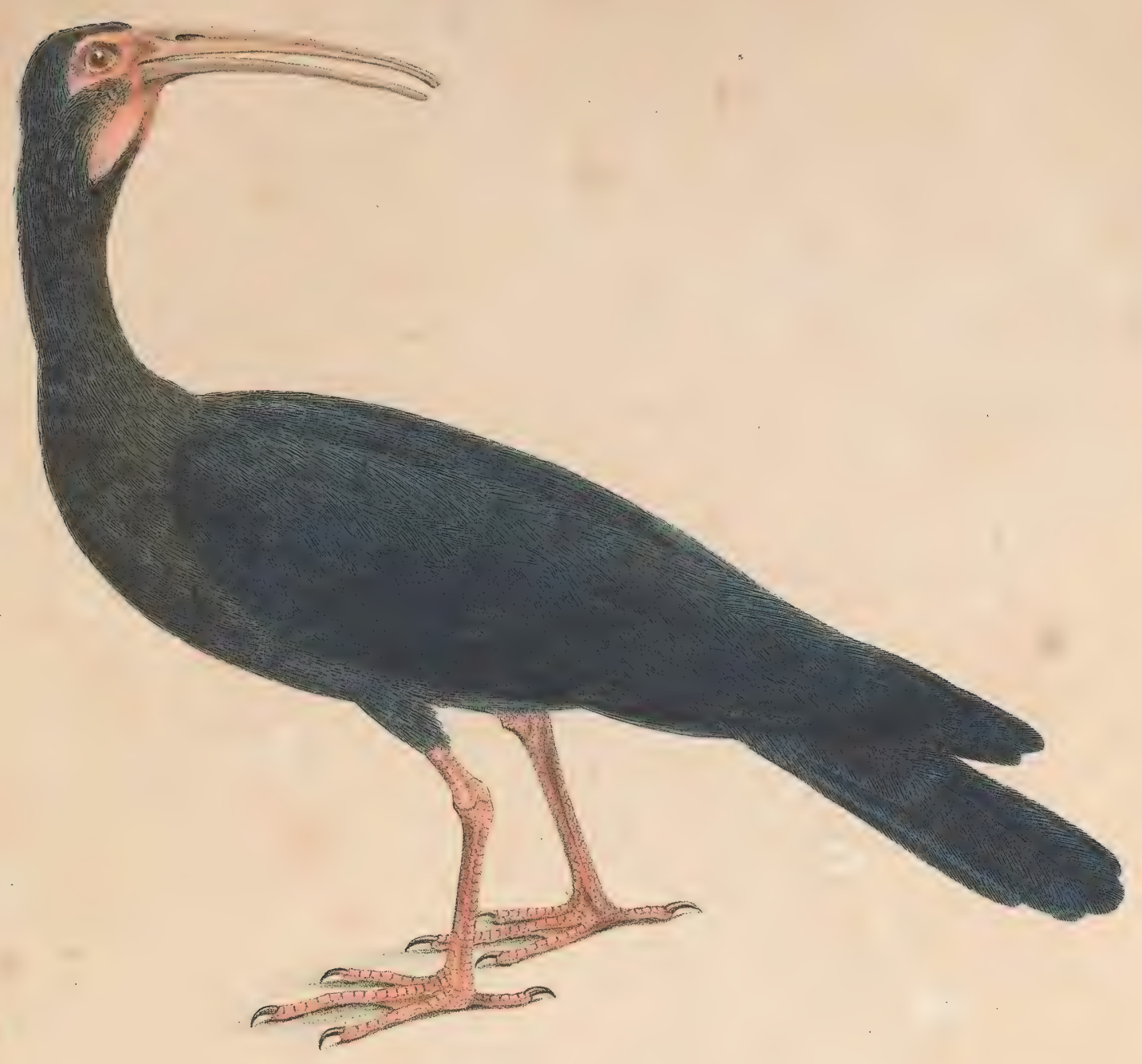

\section{tIIBISS Oxyoexcus.}

Tab: LXXXVII . 



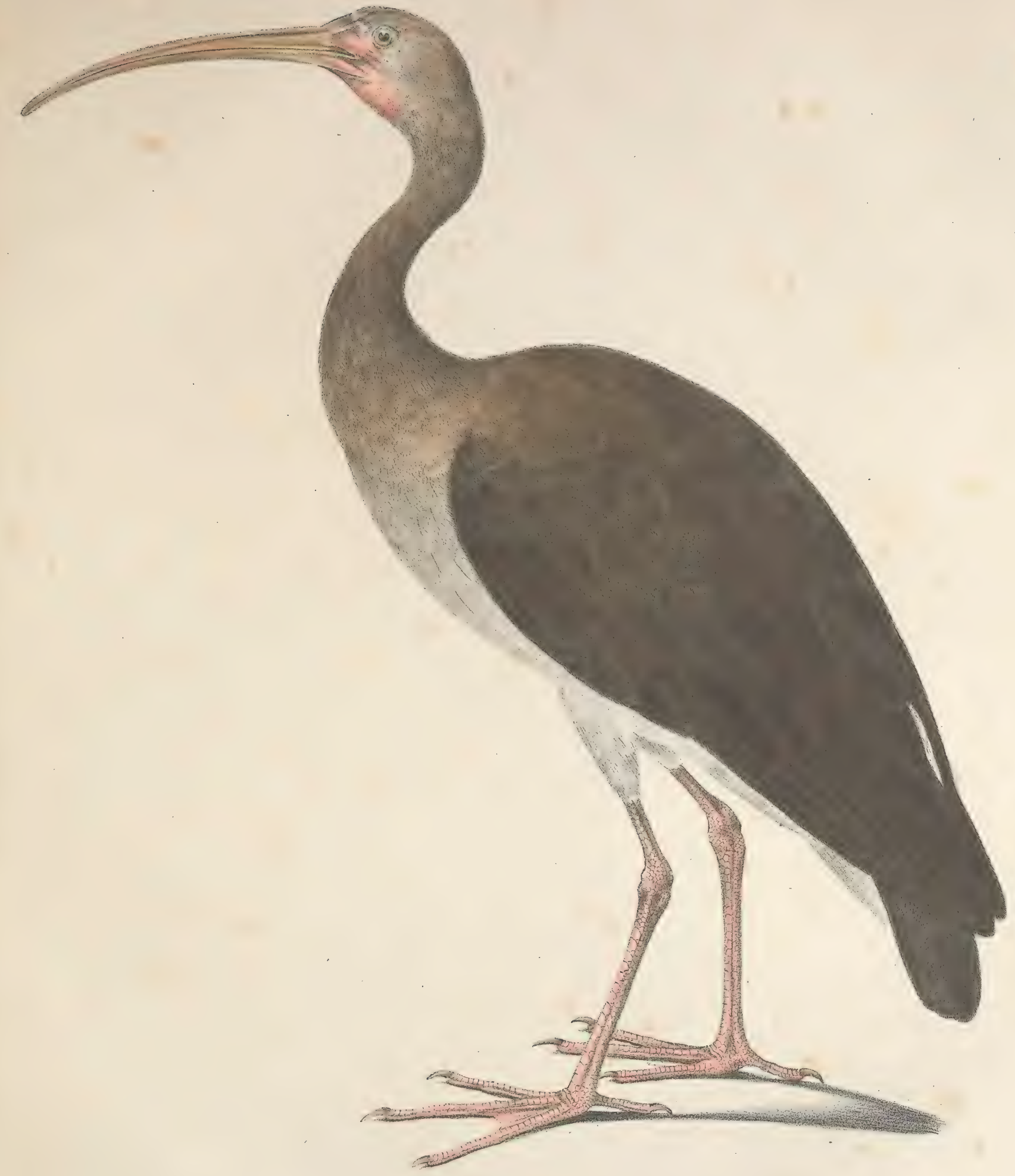

+IIBITS Lieucopyĝus.

Tab. LXXXVIII. 


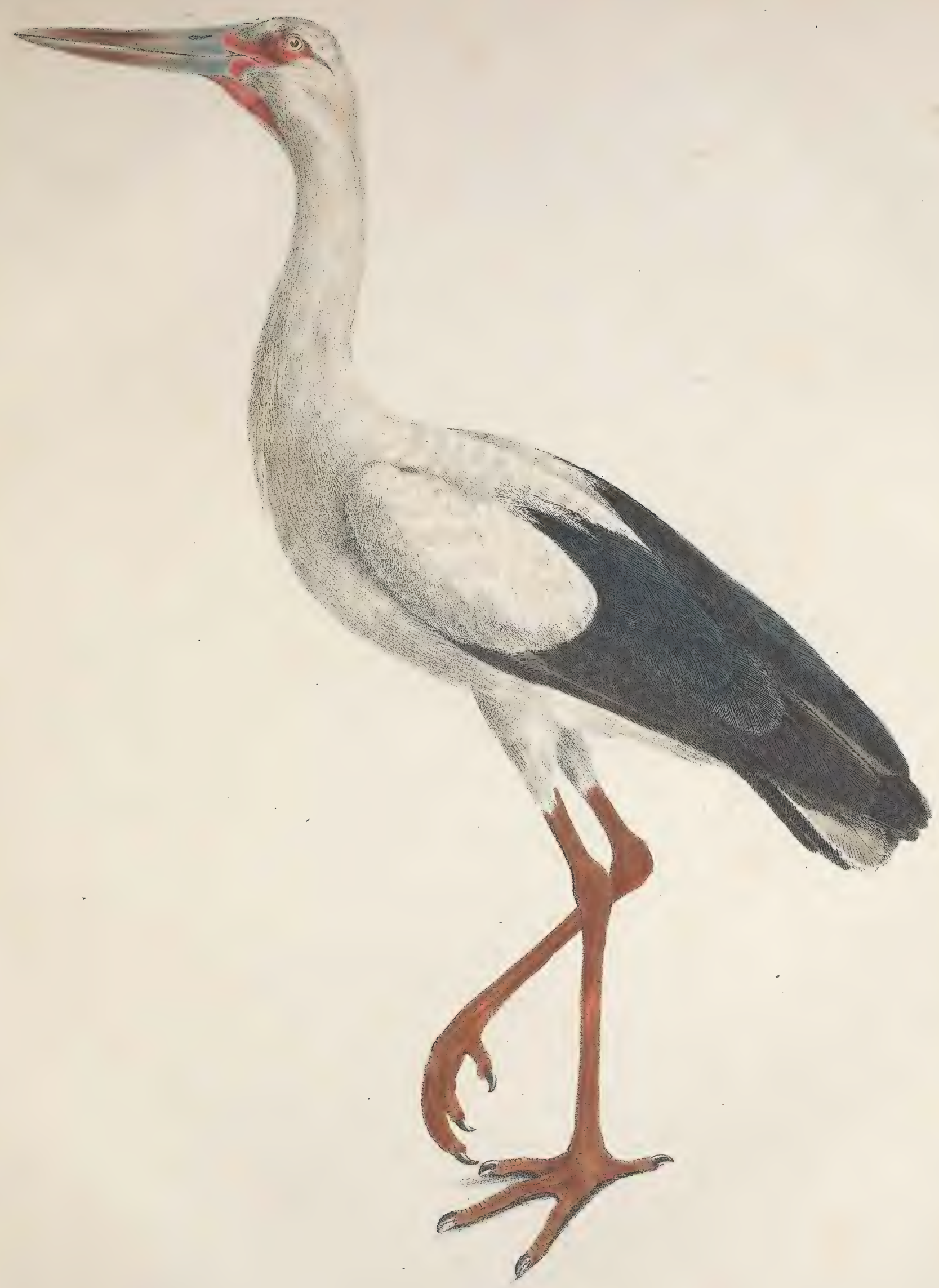

+ CIICDNIA Jaburú.

Tab:LXXXIX. 



\section{(2)}




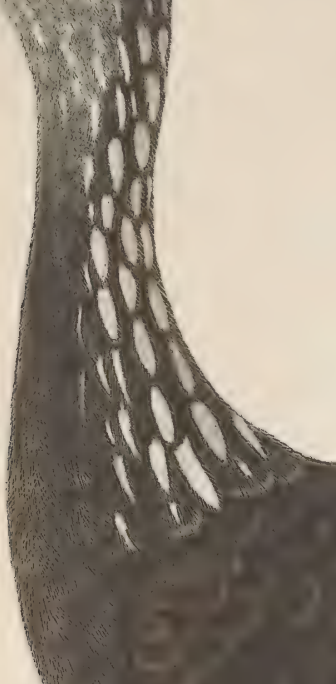


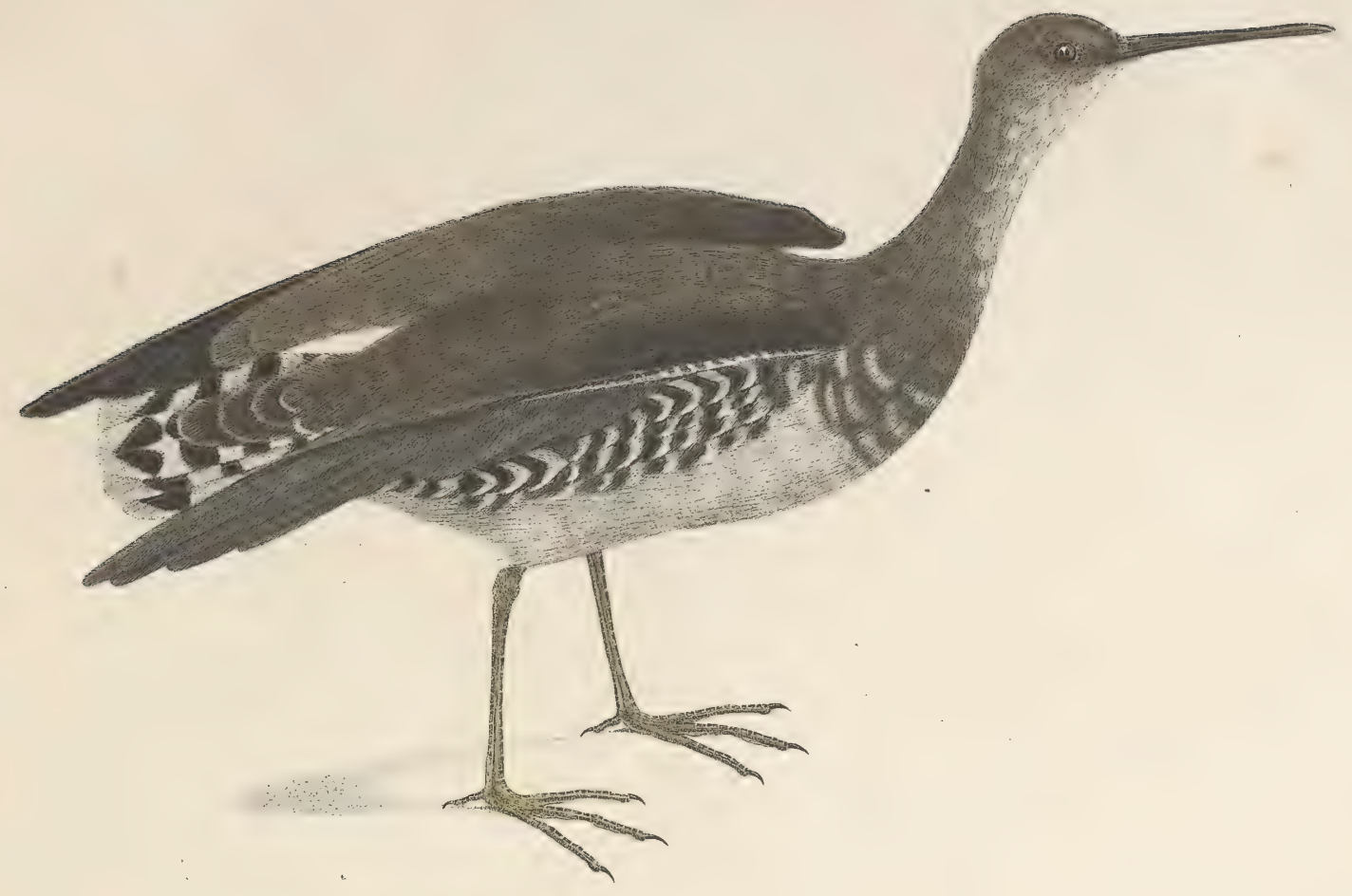

TTRINGAL macroptera.

Tab. XCII . 



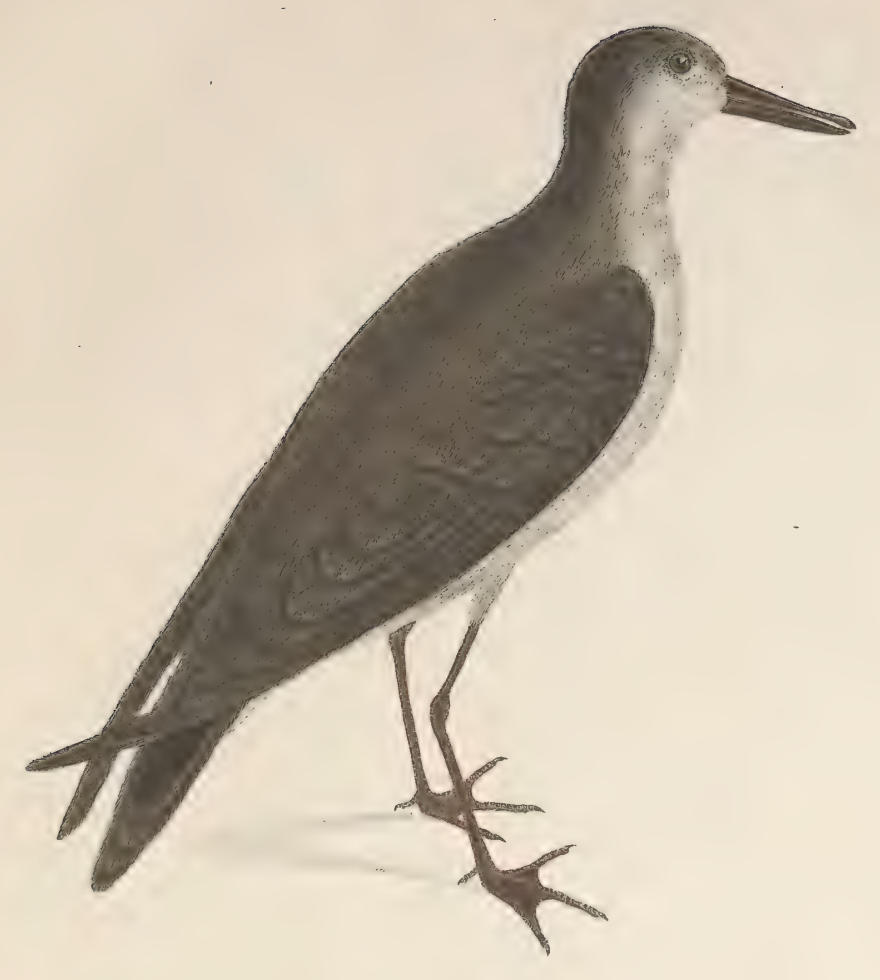

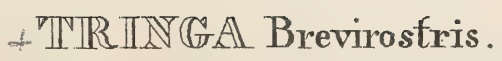

Tab.XCIII. 



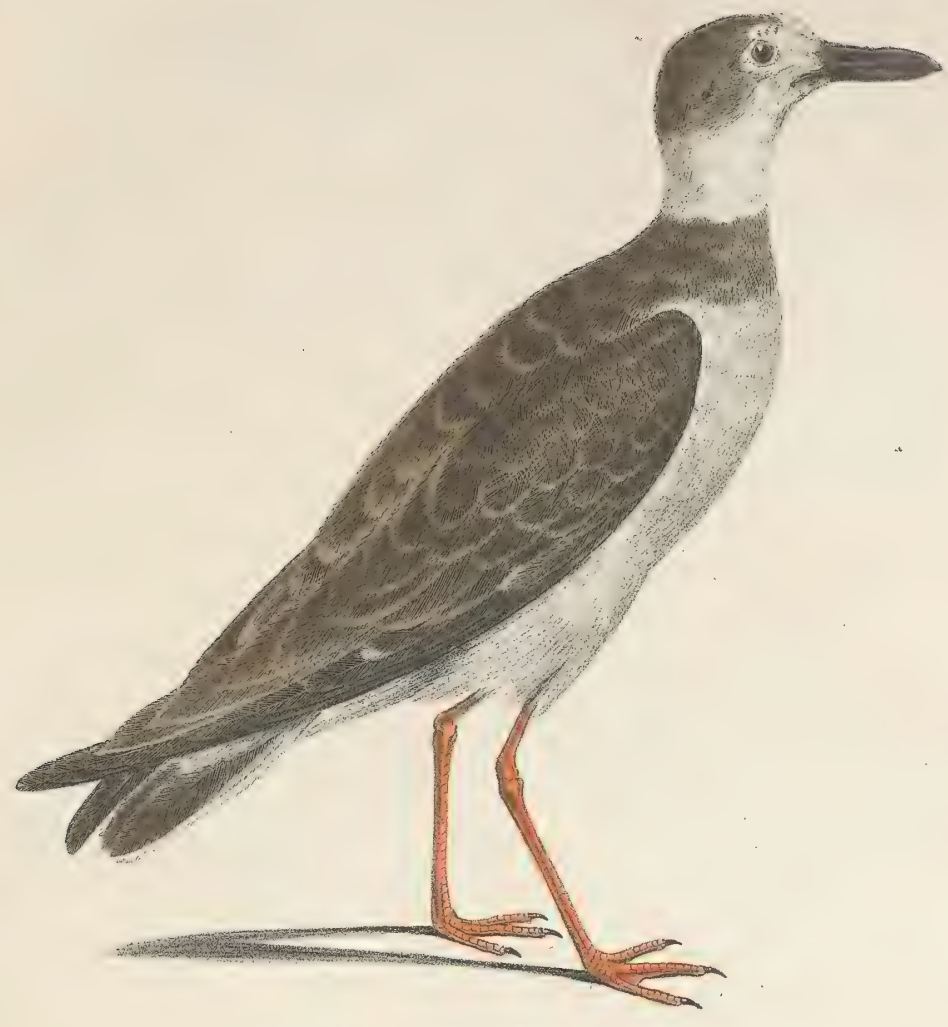

+ CCIHATRADIRIUS crassirostris.

Tab.XCIV. 



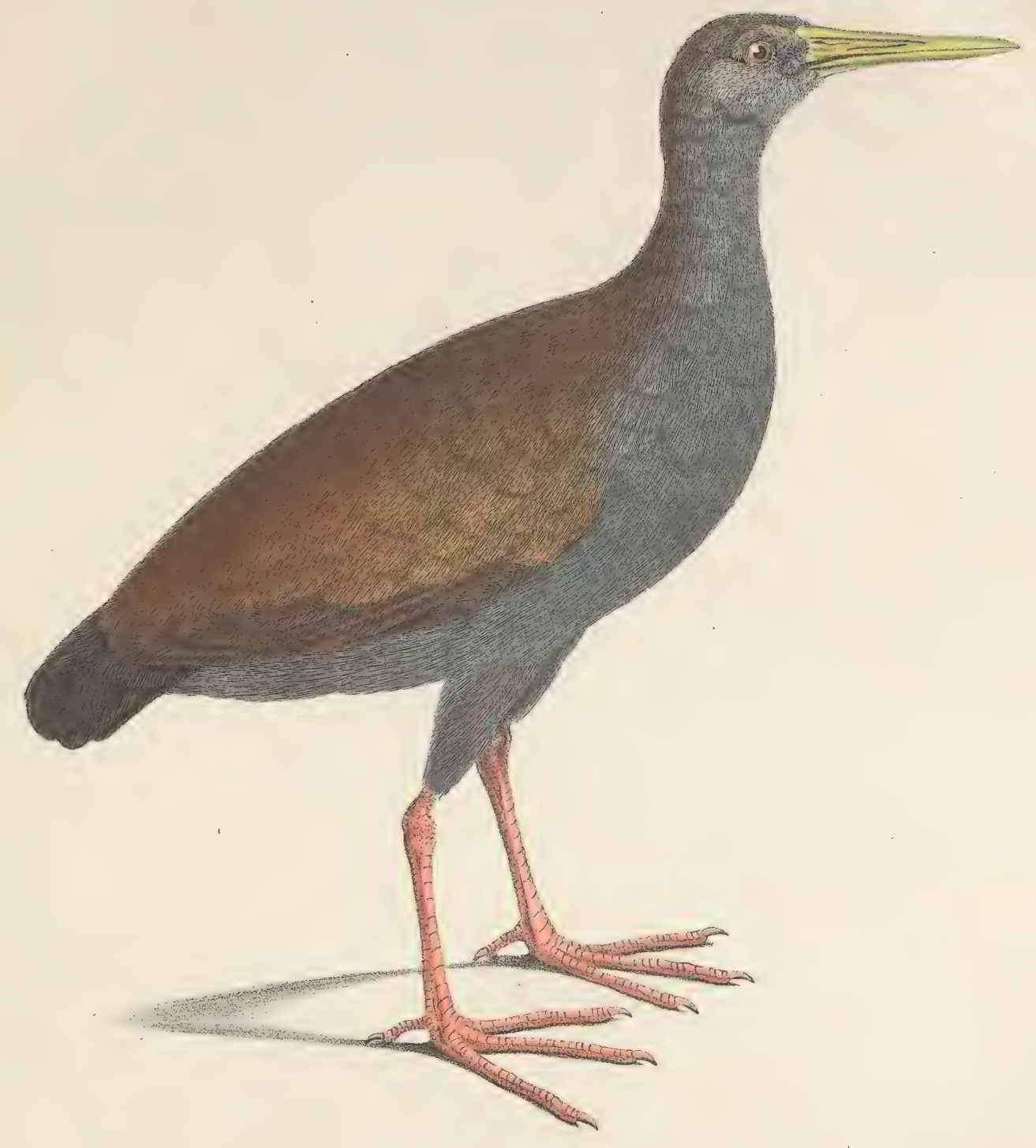

tGA GAIIINULA caesia.

Tab.XCV. 


$$
d
$$





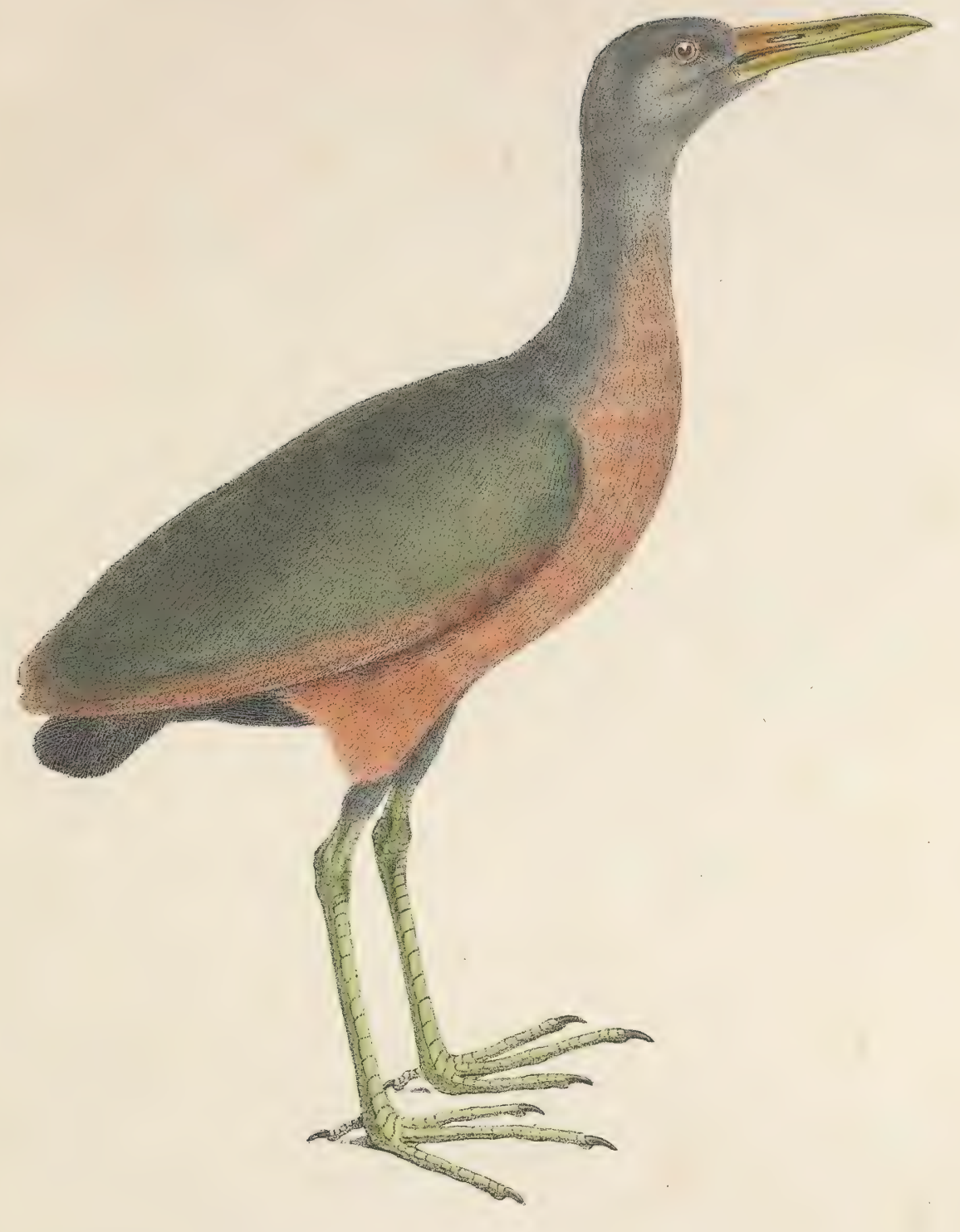

+ GA IIILIVUY ILA Manglé

Tab:XCVII. 



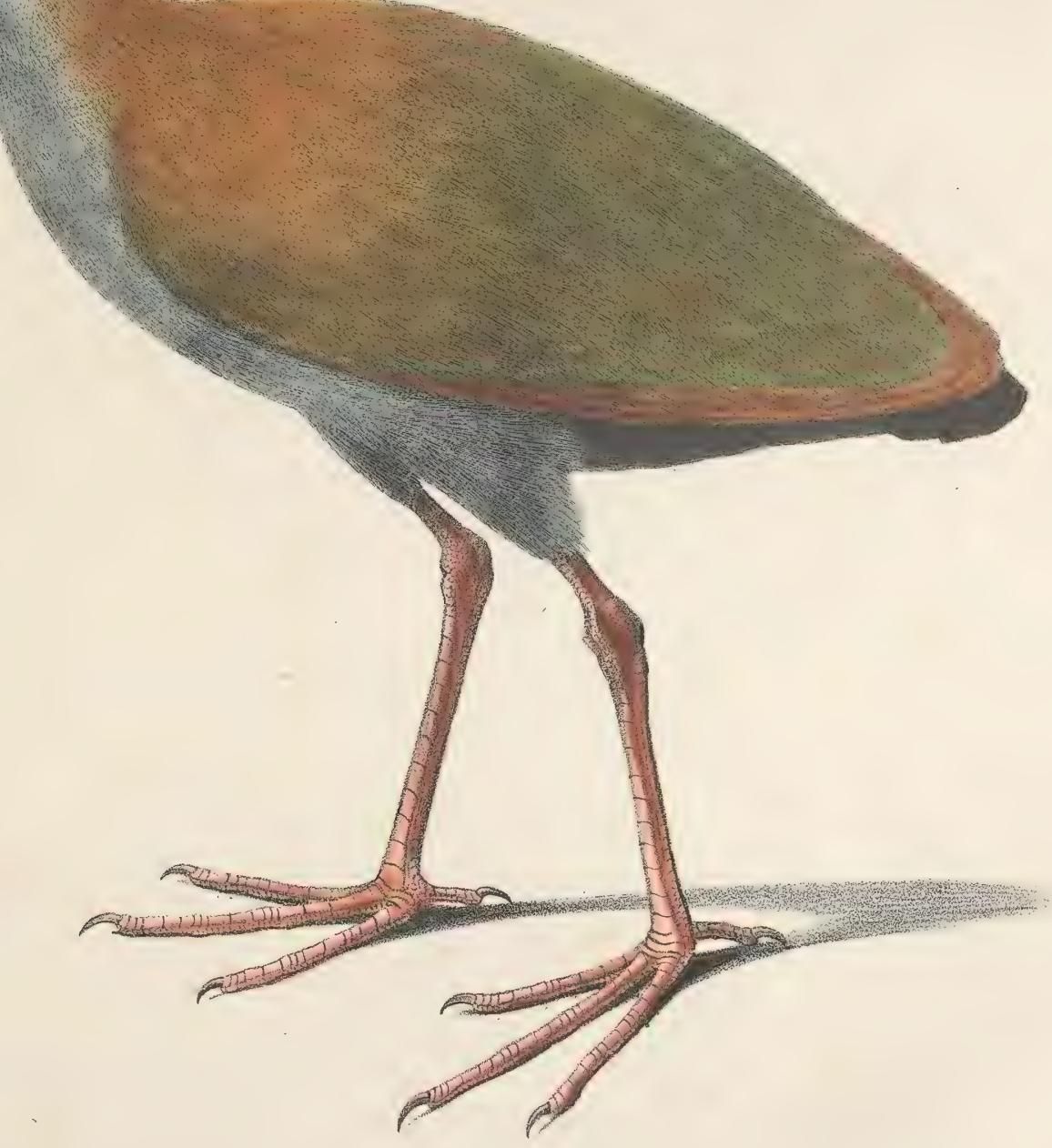

+ GATIIIIINUILA Saracura.

Tab:XCVIII . 



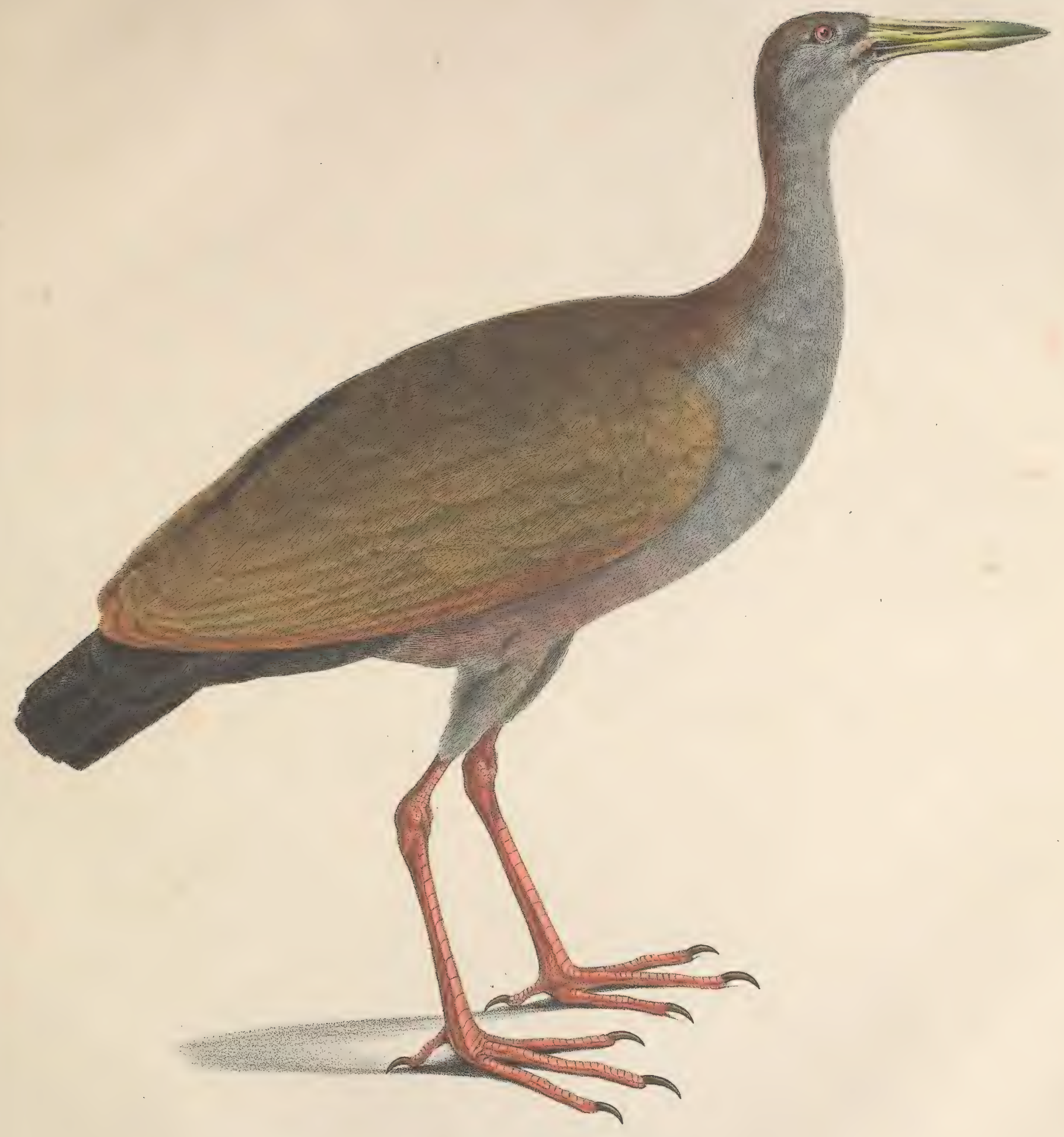

+ GA ATILIIUIIA Gigas.

Tab. XCIX. 



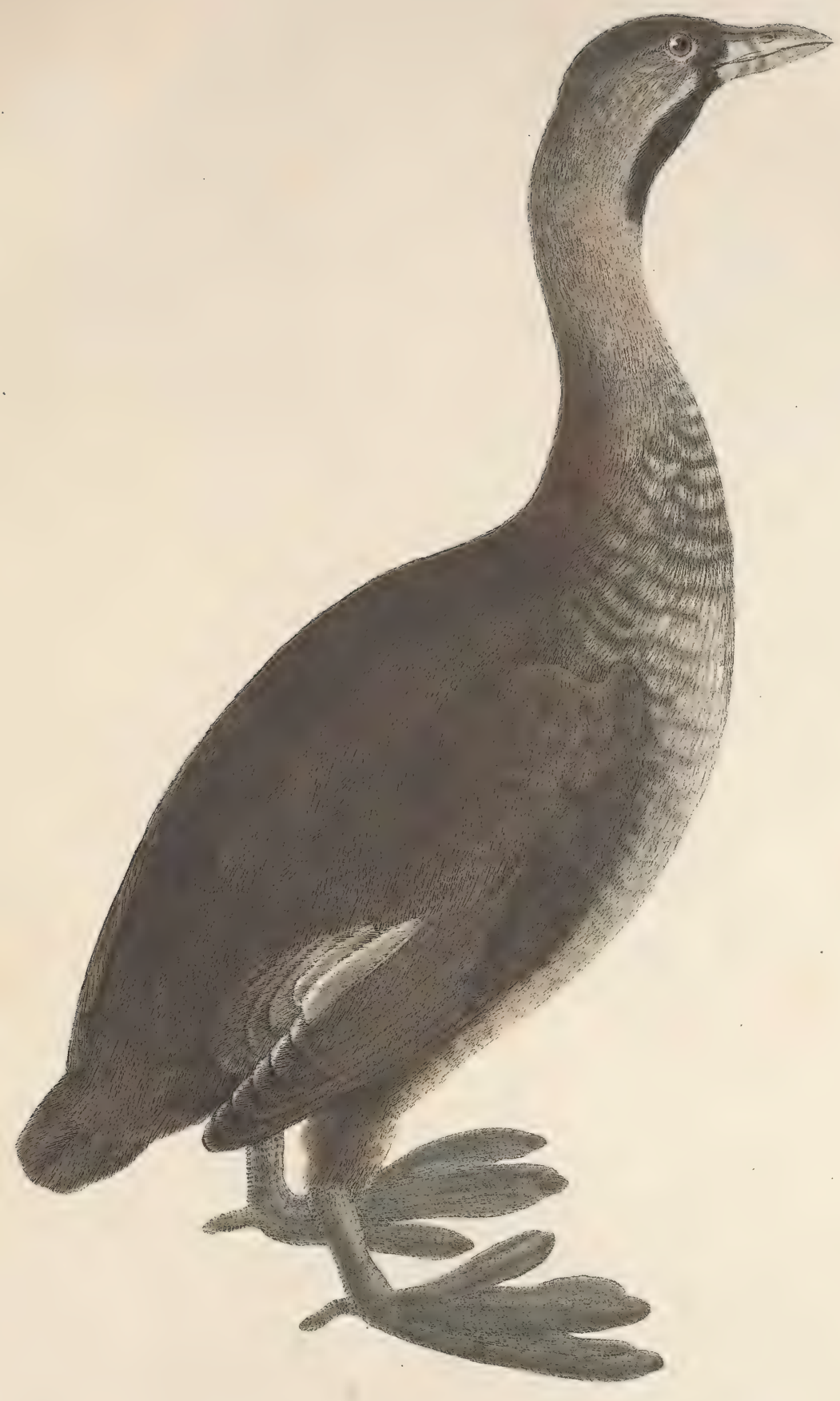

$+\mathbb{P} \mathbb{D} \mathbb{D} \mathbb{I C \mathbb { E }} \mathbb{P} S$ carolinensis.

Tab.C 



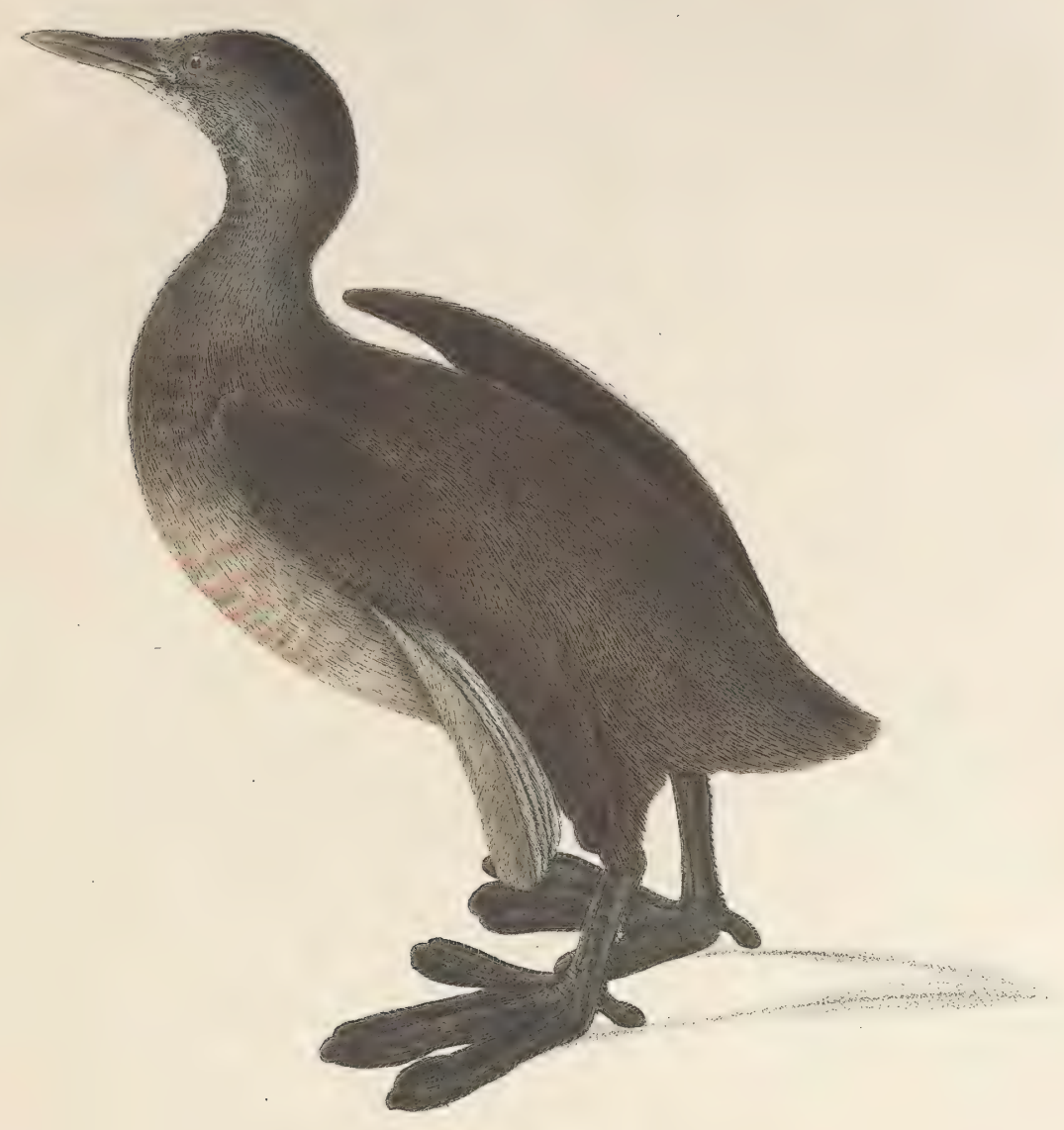

$\uparrow \mathbb{P}$ OIDICE $\mathbb{E} \mathbb{P}$ dominicus .

Tab.CI 



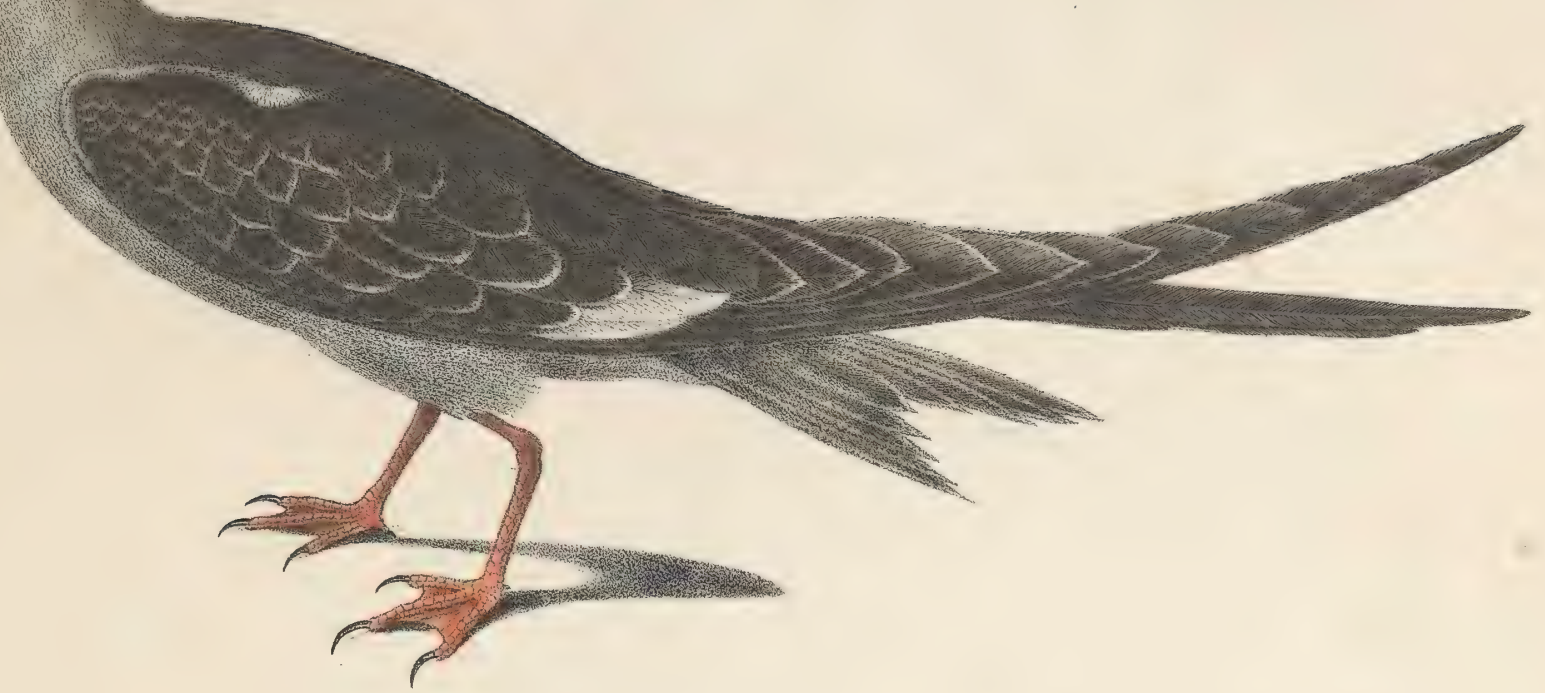

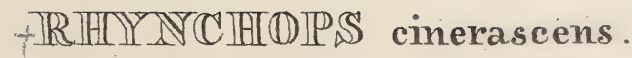

Tab.CII . 



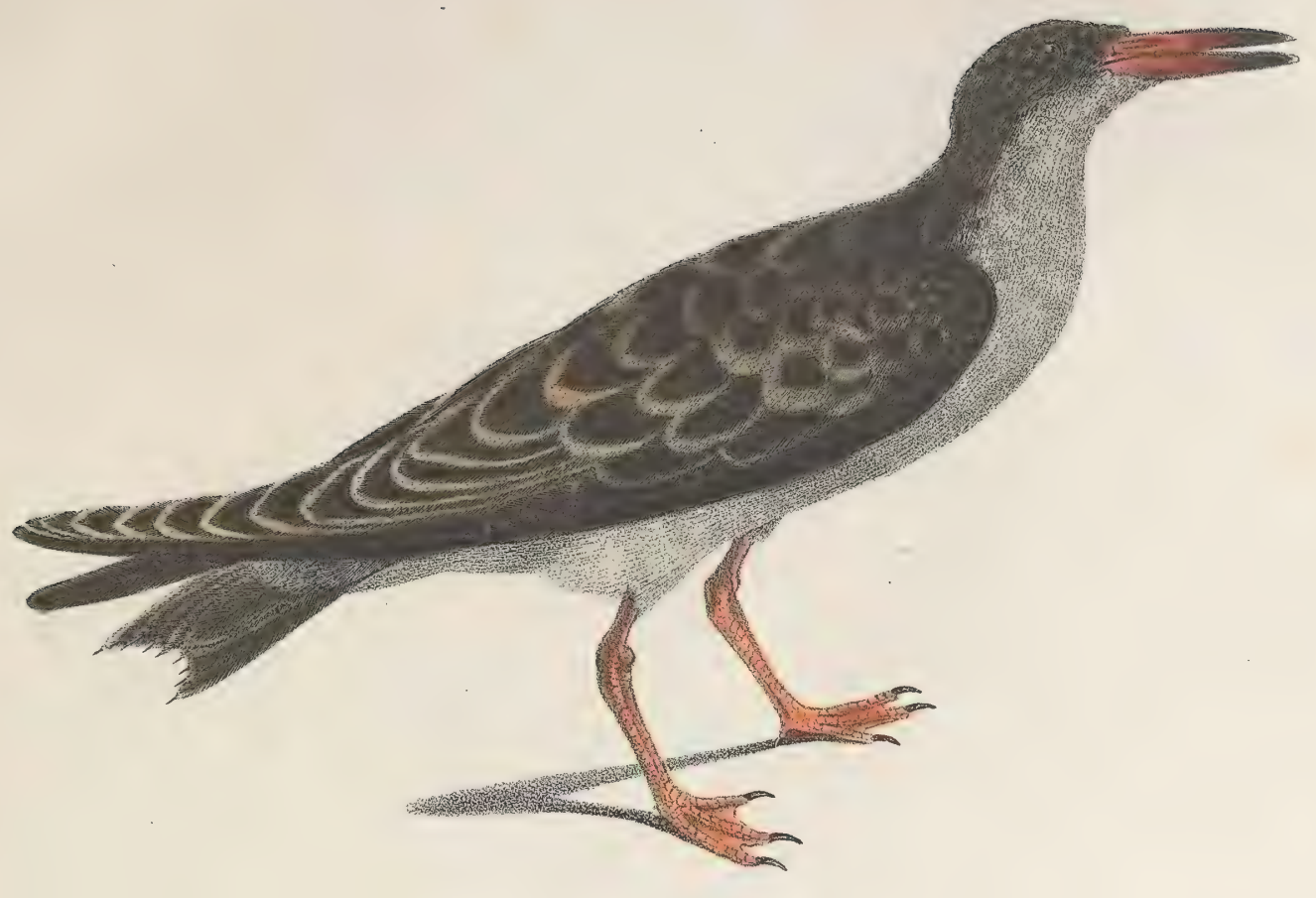

$+\mathbb{R} T H N \mathbb{N}$ CHOPS brevirostris.

Tab.CIII. 



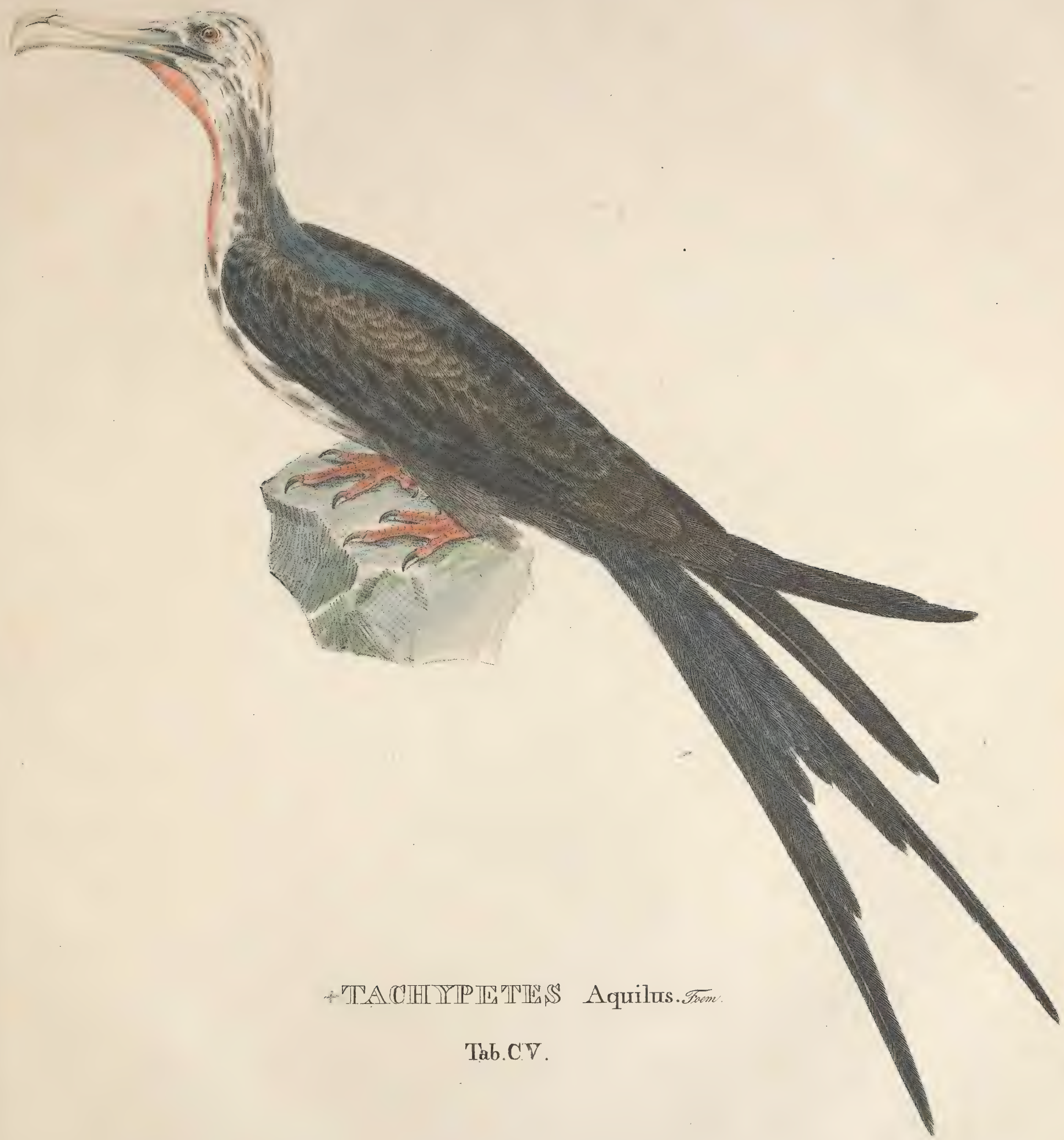




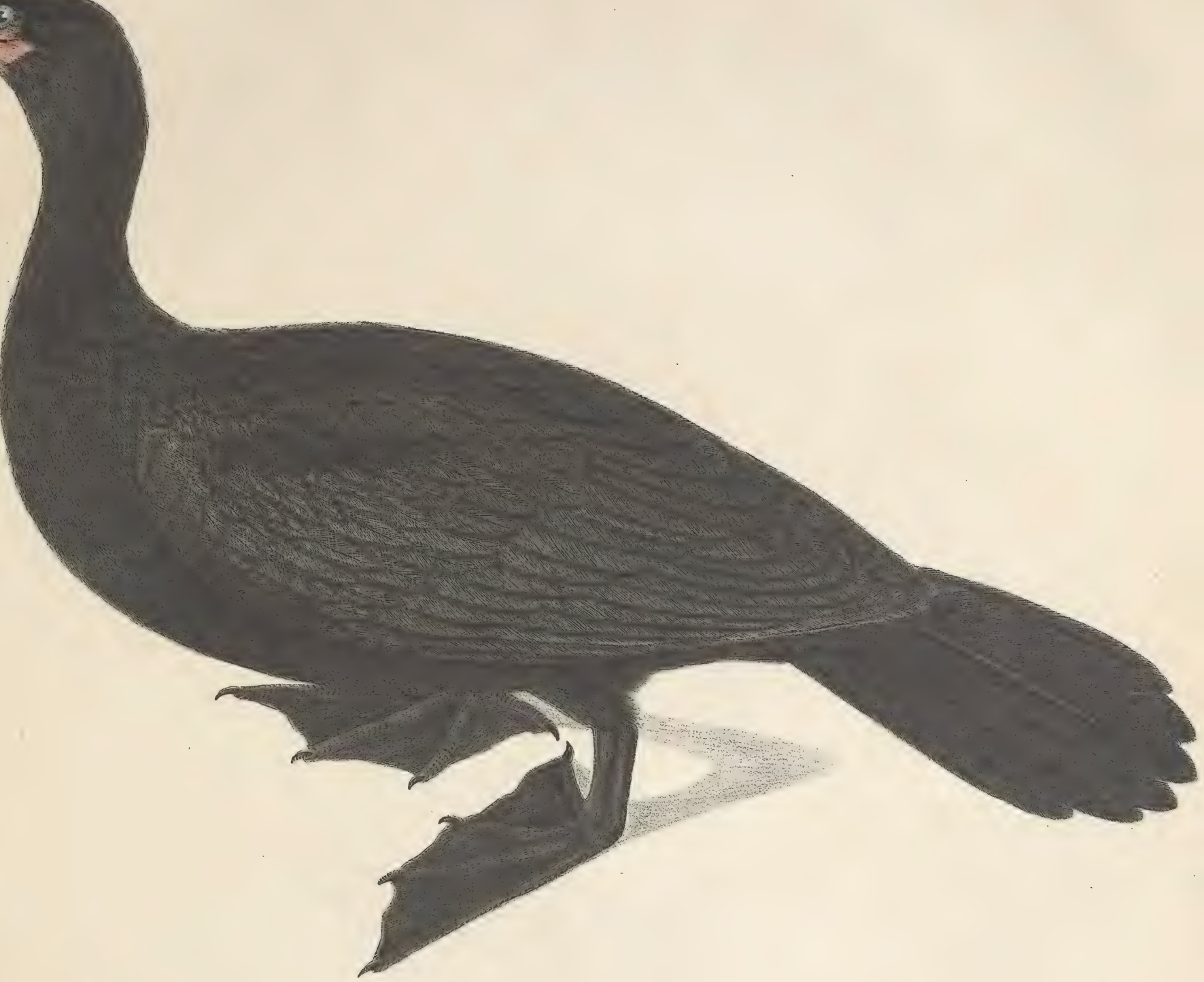

+ CA A RR IB OD brasilianus . Nas

Tab.CVI. 



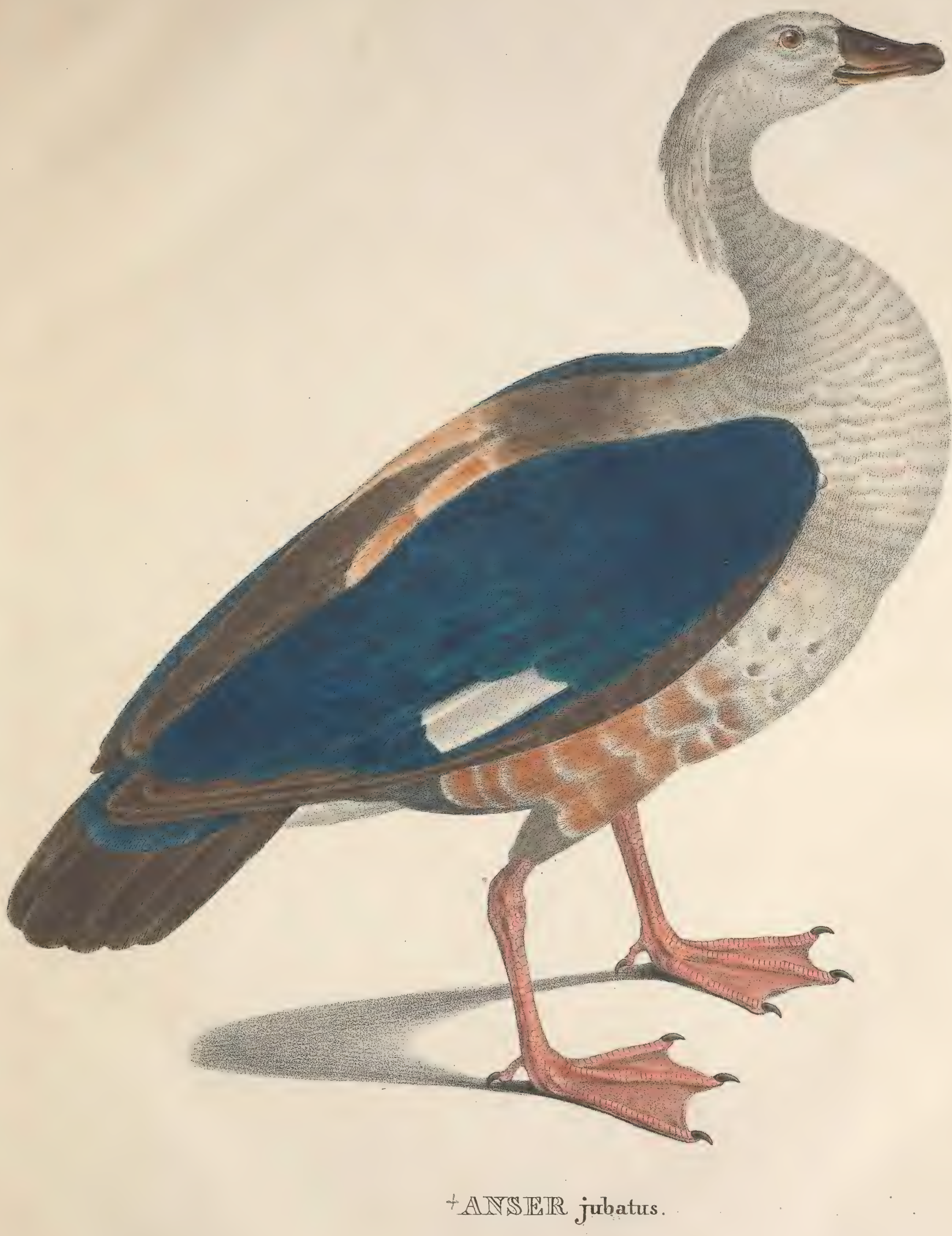

Tab. CVIII. 



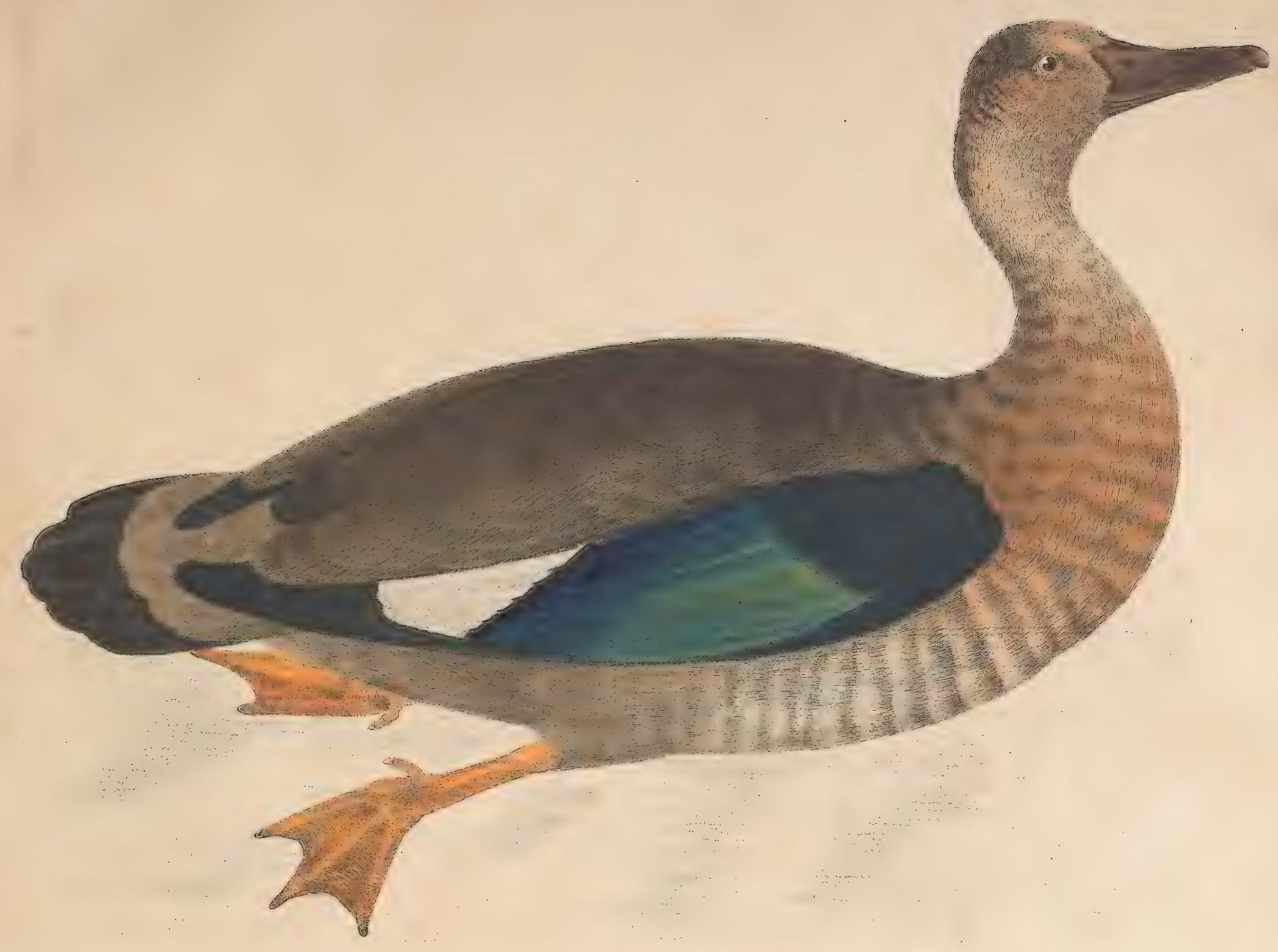

-ANAS Paturi.

Tab.CIX.

Depictree ein lapide

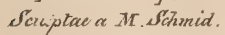


, 





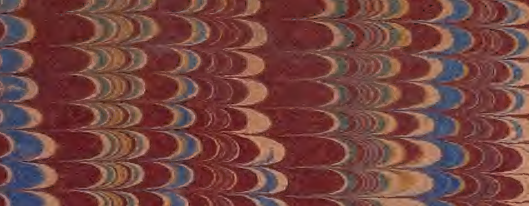

33323335

33000353

33533333353

3.

33

33

33233

3. 35313

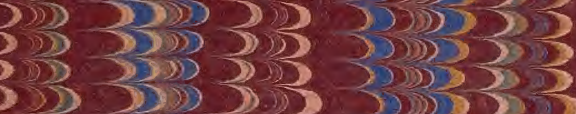

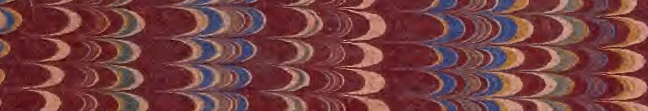

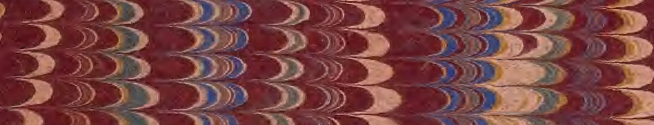

3333313

3 $33,33,33$

(5) SD)

3233

30303

33

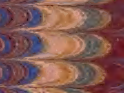

3300

$3>3$

330

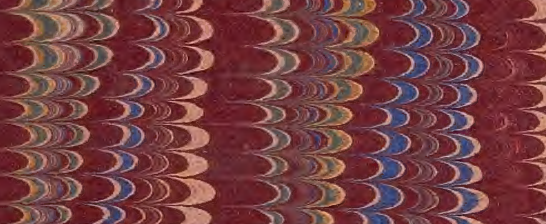

3

D)

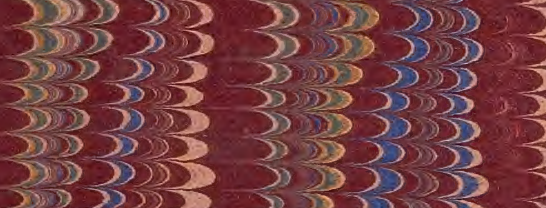

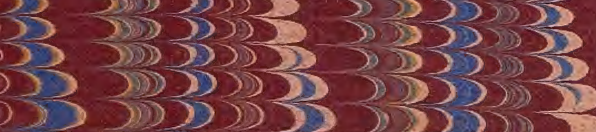

3) 23

335333

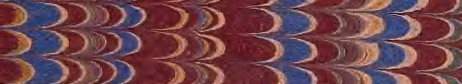

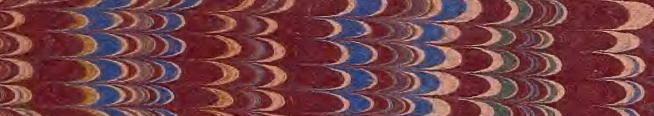

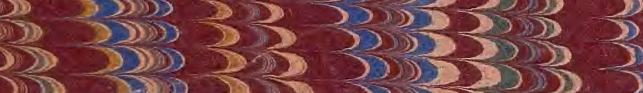

25) 35353,353

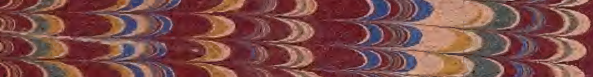

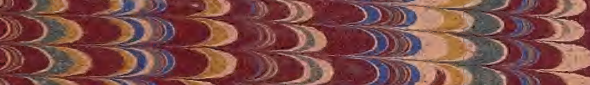

$3,3,3,3,35$

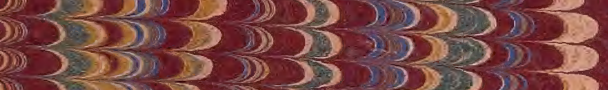

3,393

303

33

(3)

3.03

32,3

3 min

3333333

33

3

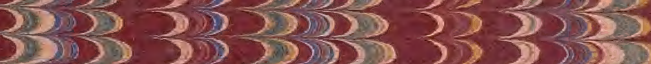

35333333

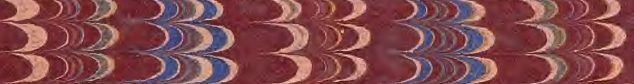

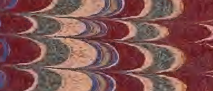

333

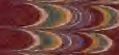

23

3

33003
32

$3,3,3$

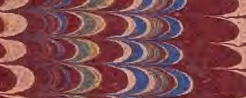

3.33

(i) 33

33

303033353

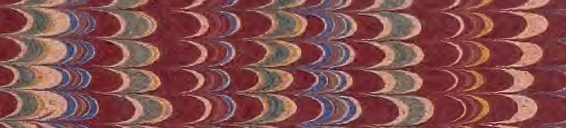

3) 3335333

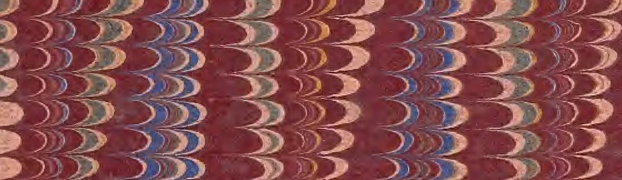

$303,353,3503$

35

25

33

3233

5) 33

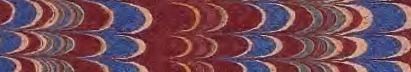

$3) 3$

33 ग) 33

in 3

330

i)

3 ) 3

3

33

(3)

3)i, 3,3

30053

(2))

32303

33333332533

32333533335

3333

33233

33253535353

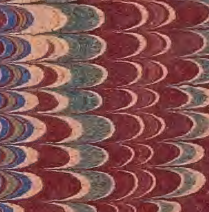

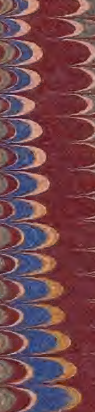

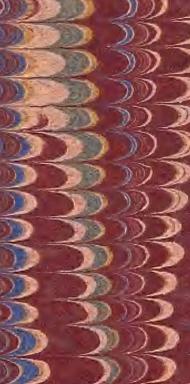

3,3

3,3

(3)

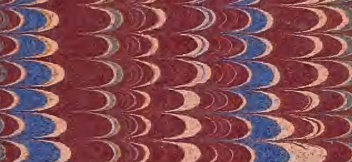

33333

333,3

33353

3 , 3

3535,3003303

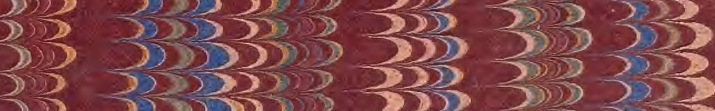

33332335

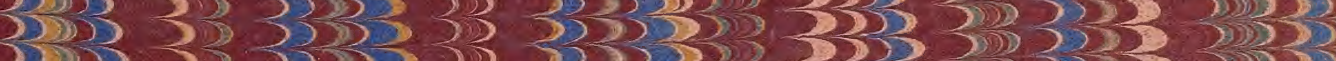

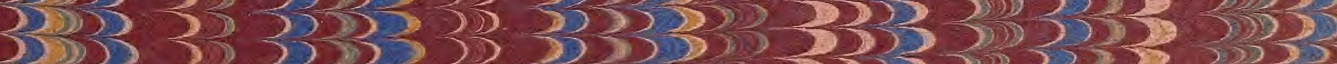




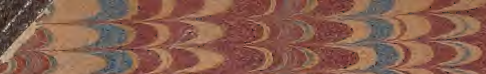

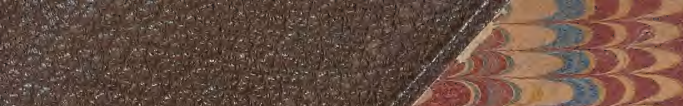

S) 22

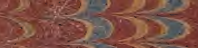

103503

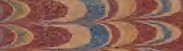

D) 21

3) 100

323

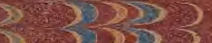

9) 3.2

0250250020

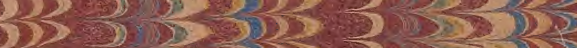

(5) 5 ( ) 55 .

()D) $>(5)$ (2)

3.202

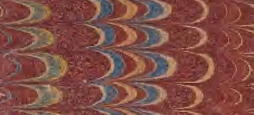

20) 2

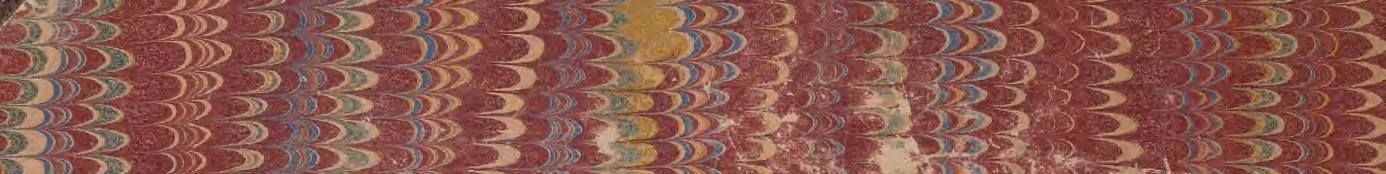

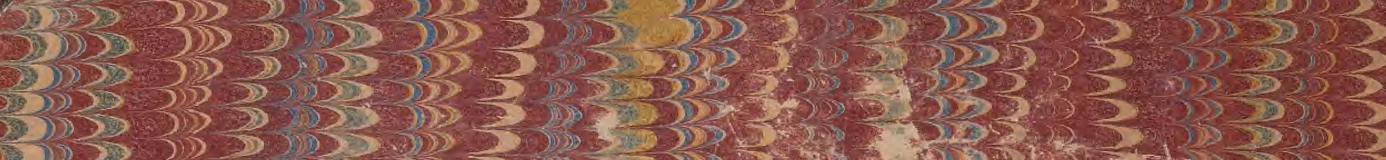

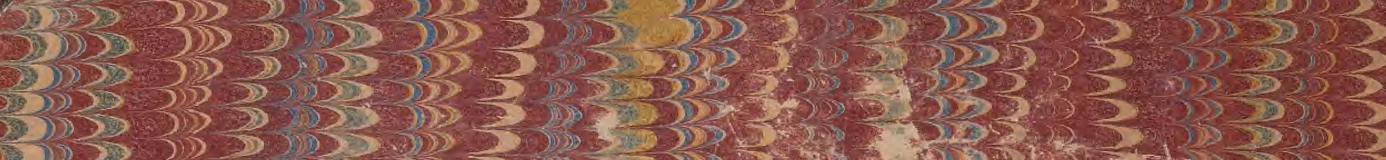

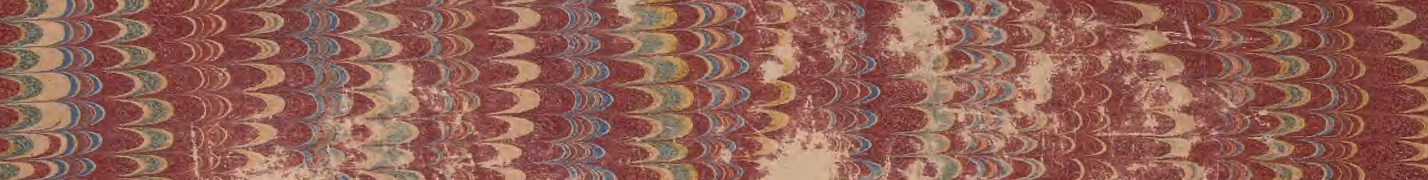

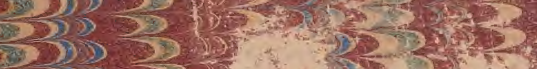

(2)

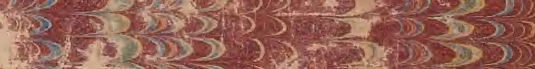

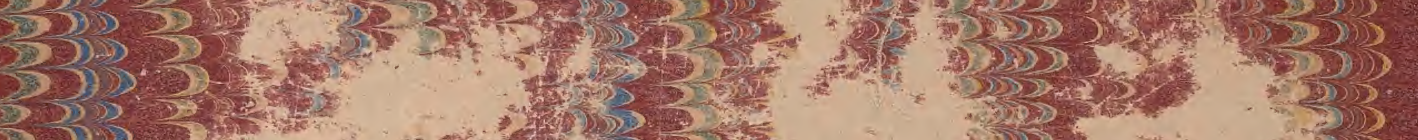

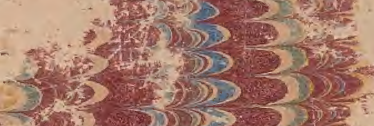

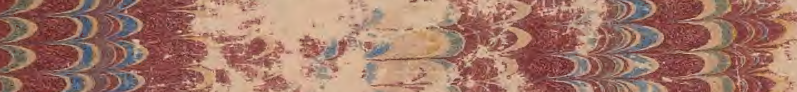

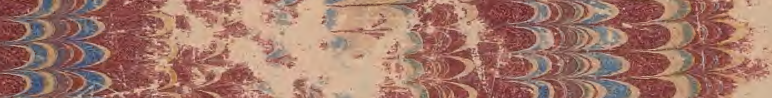

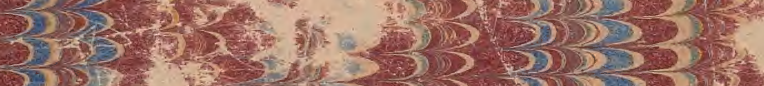

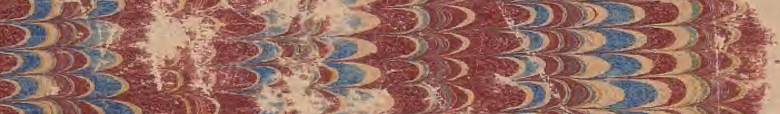

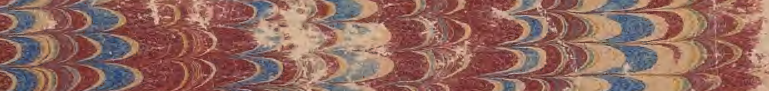

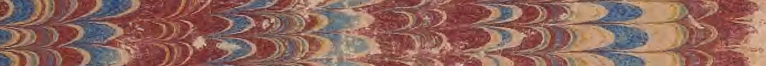

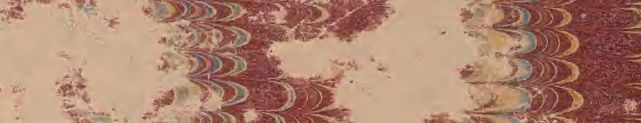

232

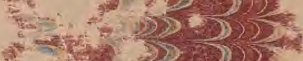

Wh $\frac{3}{3}-3$

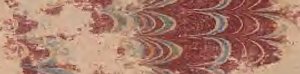

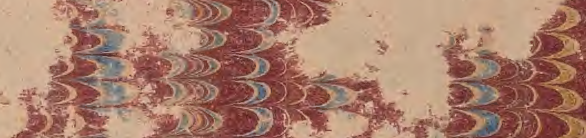

(5) )

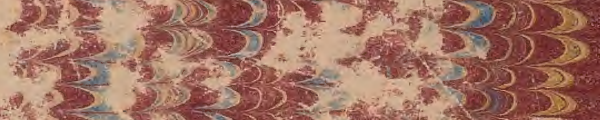

$-43,3$

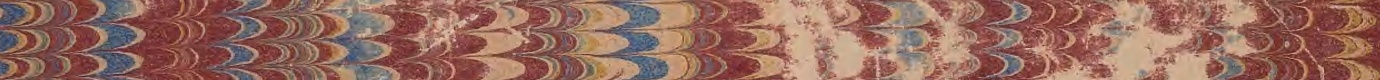

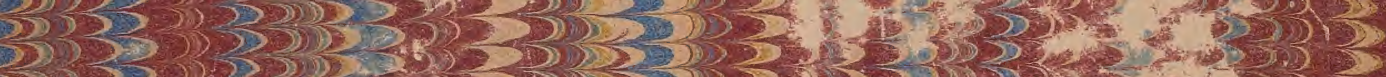

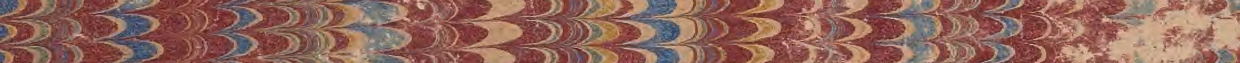

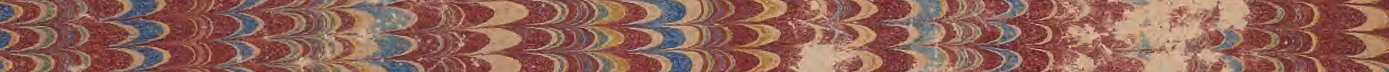

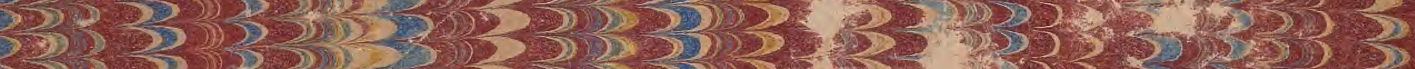

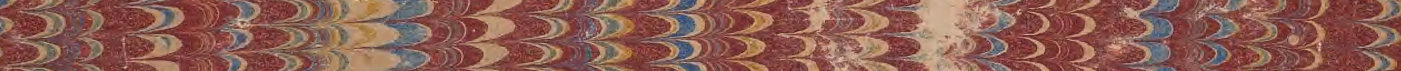

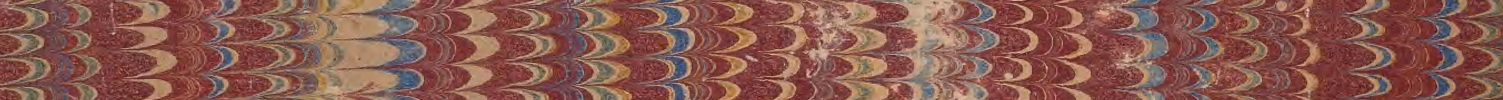

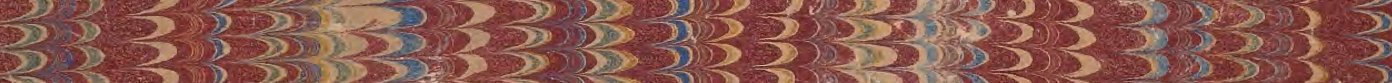

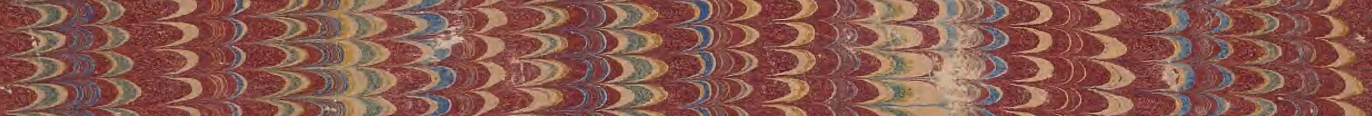

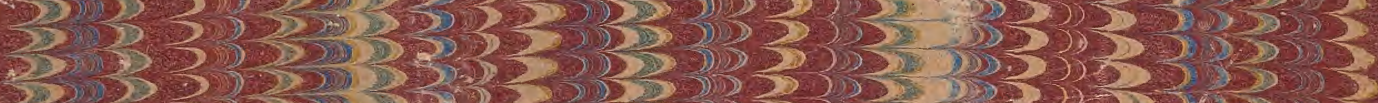

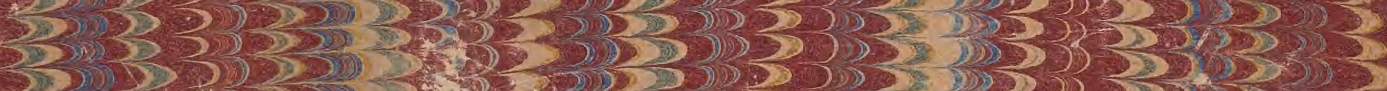

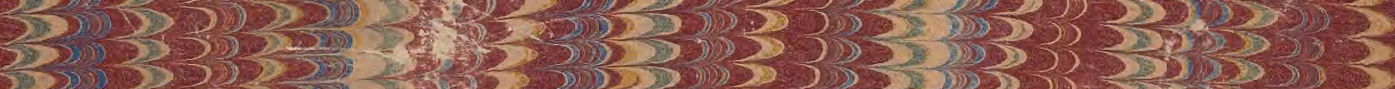

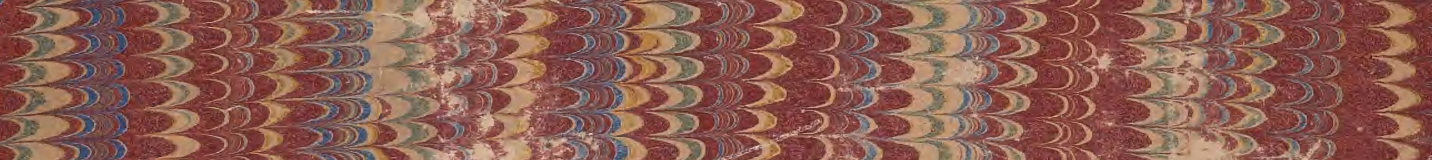

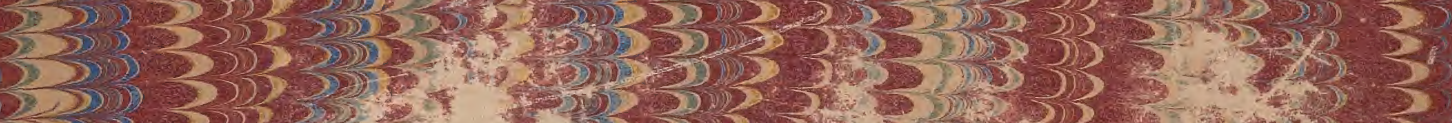

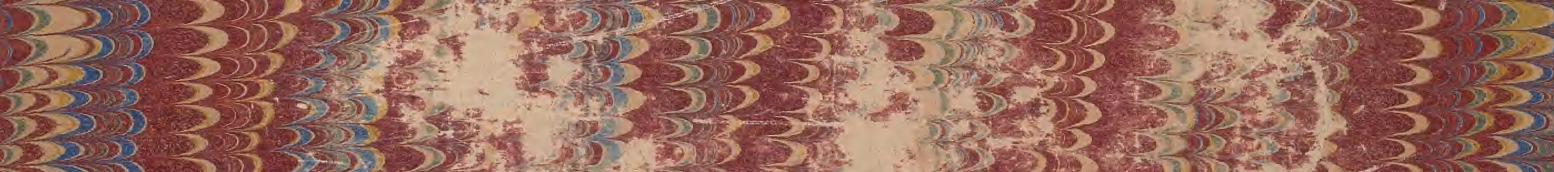

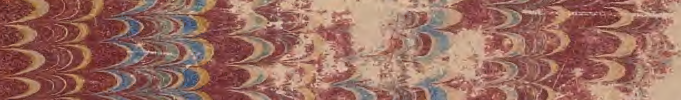

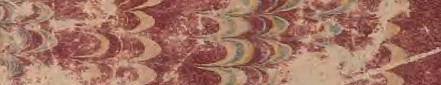

2003

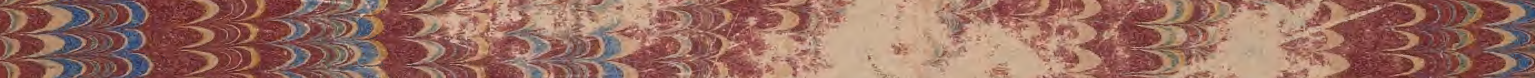

32

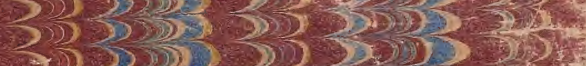

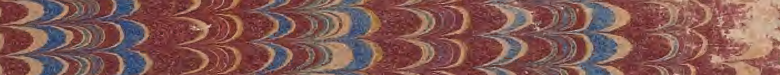

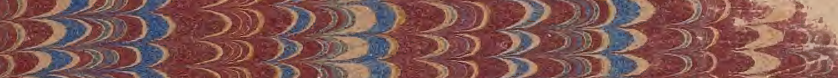

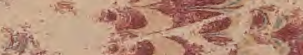

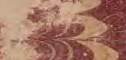

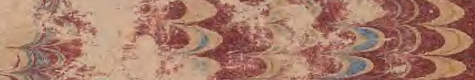

3.50 .53

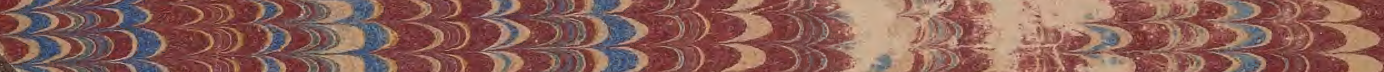

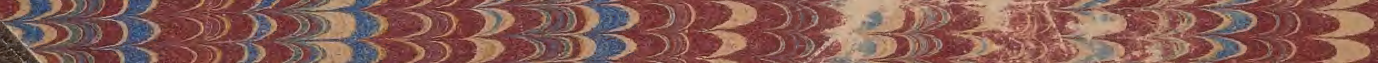

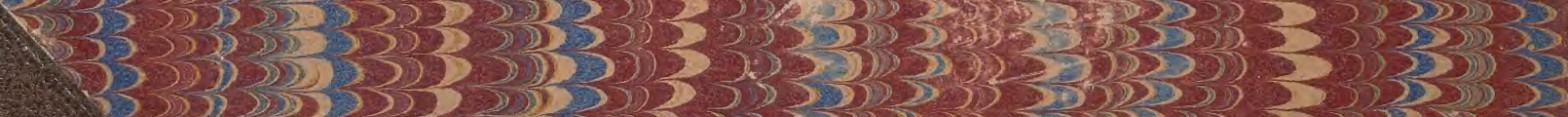

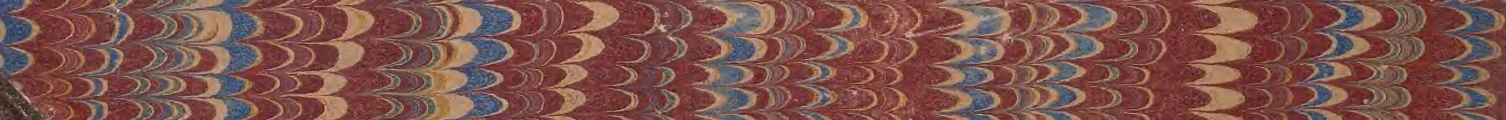

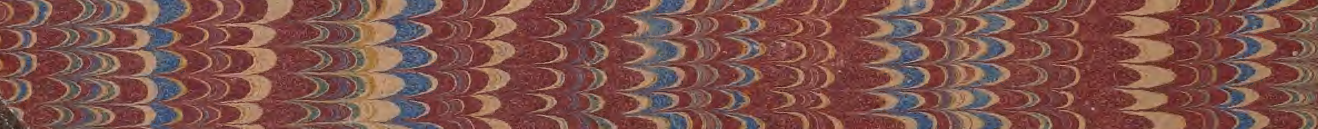

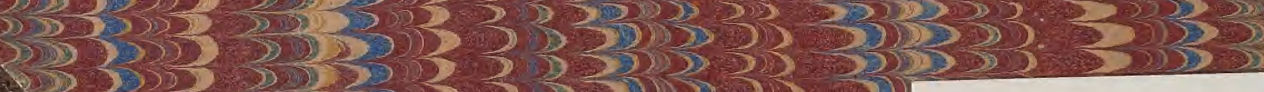

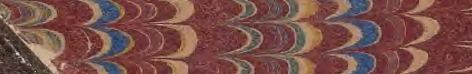
(3) $>3>30300035$
SMMTHSONAN INSTH
39088015708860

$(3,3)>3(5,3)$ (3) 